\title{
Modelos de regressão beta com erro nas variáveis
}

\author{
Jalmar Manuel Farfán Carrasco
}

TESE APRESENTADA

$\mathrm{AO}$

Instituto DE MATEMÁticA E EstatísticA

DA

UNIVERSIDADE DE SÃO PAUlO

PARA

OBTENÇÃO DO TÍTULO

$\mathrm{DE}$

Doutor EM CiÊnCIAS

\author{
Programa: Estatística \\ Orientadora: Prof. Dra. Silvia Lopes de Paula Ferrari \\ Coorientador: Prof. Dr. Reinaldo Boris Arellano-Valle
}

Durante o desenvolvimento desta tese o autor recebeu auxílio financeiro da CAPES e do CNPq, 


\section{Modelos de regressão beta com erro nas variáveis}

Esta versão definitiva da tese contém as correções e alterações sugeridas pela Comissão Julgadora durante a defesa realizada por Jalmar Manuel Farfán Carrasco em 25/5/2012.

Comissão Julgadora:

- Profa. Dra. Silvia Lopes de Paula Ferrari (orientadora) - IME-USP

- Prof. Dr. Reinaldo Boris Arellano-Valle - PUC-Chile

- Prof. Dr. Raydonal Ospina Martinez - UFPE

- Prof. Dr. Fernando Antônio da Silva Moura - UFRJ

- Prof. Dr. Mário de Castro Andrade Filho - ICMC - USP 


\section{Dedicatória}

A Deus e à virgem "Imaculada Concepção" de Ni-

nabamba - Acomayo, Perú, por abençoar meu ca-

minho dia a dia.

Aos meus pais Manuel Jacinto e Maria Jesus pelo constante apoio. A minhas adoráveis irmãs Carmen Rosa, Yeny Maria, Nancy, Jackeline e Crystel.

A Lizandra Castilho Fabio por seu carinho e apoio incondicional.

À Professora Silvia Lopes de Paula Ferrari pela orientação segura e atenciosa. 


\section{Agradecimentos}

Costuma-se dizer no final que fazer uma tese é um trabalho árduo. Acho que é muito mais que isso. Os intensos diálogos com a orientadora e co-orientador, a convivência nas disciplinas de formação, as conversas nos corredores e na sala do café, as participações nos congressos, nas conferências, as trocas de correios eletrônicos, o apoio recebido nos momentos de angústia, as sugestões recebidas e o árduo trabalho de revisão constituem um todo coletivo deste trabalho.

Cada uma destas observações tem seu mérito. Agradecer é um gesto mínimo de reconhecimento perante a intensidade do carinho e gratidão que sinto por todos aqueles que viram neste trabalho a importância de sê-lo.

Reitero minha gratidão à Professora Silvia Lopes de Paula Ferrari, pelos anos de orientação acadêmica e pessoal e pela paciência no acompanhamento deste trabalho.

Das contribuições do Professor Reinaldo Boris Arellano Valle, pela oportunidade de visitá-lo no Departamento de Estatística da Universidad Católica de Chile para iniciar este trabalho.

Muito obrigado a meus mestres e amigos, como os considero, aos professores Edwin Moisés Marcos Ortega e Gauss Cordeiro.

Meus sinceros agradecimentos aos professores Antônio Carlos Pedroso de Lima, Airlane Pereira Alencar, Lúcia Pereira Barroso, Gilberto Alvarenga Paula e Heleno Bolfarine, que contribuíram para minha formação com as disciplinas que cursei durante os primeiros anos de doutorado.

Meus agradecimentos a Olga Satomi Yoshida, gerente de projetos do Centro de Metrologia de Fluidos do Instituto de Pesquisas Tecnológicas (IPT) por brindar-me amizade, tempo e assessoria técnica.

Em termos institucionais, foram fundamentais as bolsas de estudos da Coordenação de Aperfeiçoamento de Pessoal de Nível Superior (CAPES) e do Conselho Nacional de Desenvolvimento Científico e Tecnológico (CNPq); o apoio financeiro do Instituto de Matemática e Estatística (IME) para participar dos diferentes eventos científicos, o Fondo Nacional de Desarrollo Científico e Tecnológico (FONDECYT)-Chile, que apoiou nas despesas da minha estadia no Chile e a Superintendência de Assistência Social (SAS), que me brindou com moradia desde o primeiro dia em que cheguei a São Paulo.

Agradeço a meus colegas de apartamento Carlos Carrasco Arbieto e Daniel Alonso da Silva por compartilharem momentos gratos e harmoniosos durante estes quatro anos.

Agradeço carinhosamente a meus amigos e colegas do instituto Gustavo Pereira, Luz Marina Gomes, Nubia Estefano, Artur José Lemonte, Alexandre Patriota, Tiago Maia Magalhães, Rafael Farias, Michel Montoril, Amanda Gomes dos Santos, Gerard John Alva Morales, Jorge Luis Torrejon, Marco Riquelme, Tiago Moreira Vargas, Giovana Fumes e Eliane Cantinho Pinheiro.

Agradeço a meus familiares pelo estímulo e apoio constante, sobretudo pela compreensão de muitos anos de ausência.

Meus agradecimentos sinceros aos professores Raydonal Ospina Martinez, Fernando Antônio da 
Silva Moura e Mário de Castro Andrade Filho que conformaram a banca examinadora.

Enfim, a todos que de algum modo se fizeram presentes nessa caminhada, muito obrigado!!! 


\section{Resumo}

\section{Modelos de regressão beta com erro nas variáveis}

Neste trabalho de tese propomos um modelo de regressão beta com erros de medida. Esta proposta é uma área inexplorada em modelos não lineares na presença de erros de medição. Abordamos metodologias de estimação, como máxima verossimilhança aproximada, máxima pseudo-verossimilhança aproximada e calibração da regressão. O método de máxima verossimilhança aproximada determina as estimativas maximizando diretamente o logaritmo da função de verossimilhança. $\mathrm{O}$ método de máxima pseudo-verossimilhança aproximada é utilizado quando a inferência em um determinado modelo envolve apenas alguns mas não todos os parâmetros. Nesse sentido, dizemos que o modelo apresenta parâmetros de interesse como também de perturbação. Quando substituímos a verdadeira covariável (variável não observada) por uma estimativa da esperança condicional da variável não observada dada a observada, o método é conhecido como calibração da regressão. Comparamos as metodologias de estimação mediante um estudo de simulação de Monte Carlo. Este estudo de simulação evidenciou que os métodos de máxima verossimilhança aproximada e máxima pseudo-verossimilhança aproximada tiveram melhor desempenho frente aos métodos de calibração da regressão e naïve (ingênuo). Utilizamos a linguagem de programação ox (Doornik, 2011) como suporte computacional.

Encontramos a distribuição assintótica dos estimadores, com o objetivo de calcular intervalos de confiança e testar hipóteses, tal como propõem Carroll et al. (2006, Seção A.6.6), Guolo (2011) e Gong e Samaniego (1981). Ademais, são utilizadas as estatísticas da razão de verossimilhanças e gradiente para testar hipóteses. Num estudo de simulação realizado, avaliamos o desempenho dos testes da razão de verossimilhanças e gradiente.

Desenvolvemos técnicas de diagnóstico para o modelo de regressão beta com erros de medida. Propomos o resíduo ponderado padronizado tal como definem Espinheira et al. (2008b) com o objetivo de verificar as suposições assumidas ao modelo e detectar pontos aberrantes. Medidas de influência global, tais como a distância de Cook generalizada e o afastamento da verossimilhança, são utilizadas para detectar pontos influentes. Além disso, utilizamos a técnica de influência local conformal sob três esquemas de perturbação (ponderação de casos, perturbação da variável resposta e perturbação da covariável com e sem erros de medida). Aplicamos nossos resultados a dois conjuntos de dados reais para exemplificar a teoria desenvolvida. Finalmente, apresentamos algumas conclusões e possíveis trabalhos futuros.

Palavras-chave: Modelo de regressão beta, modelo com erros de medida, estimação por máxima verossimilhança aproximada, estimação por máxima pseudo-verossimilhança aproximada, calibração da regressão, análise de diagnóstico. 


\section{Abstract}

\section{Beta regression model with measurement error}

In this thesis, we propose a beta regression model with measurement error. Among nonlinear models with measurement error, such a model has not been studied extensively. Here, we discuss estimation methods such as maximum likelihood, pseudo-maximum likelihood, and regression calibration methods. The maximum likelihood method estimates parameters by directly maximizing the logarithm of the likelihood function. The pseudo-maximum likelihood method is used when the inference in a given model involves only some but not all parameters. Hence, we say that the model under study presents parameters of interest, as well as nuisance parameters. When we replace the true covariate (observed variable) with conditional estimates of the unobserved variable given the observed variable, the method is known as regression calibration. We compare the aforementioned estimation methods through a Monte Carlo simulation study. This simulation study shows that maximum likelihood and pseudo-maximum likelihood methods perform better than the calibration regression method and the naïve approach. We use the programming language ox (Doornik, 2011) as a computational tool.

We calculate the asymptotic distribution of estimators in order to calculate confidence intervals and test hypotheses, as proposed by Carroll et al. (2006, Section A.6.6), Guolo (2011) and Gong e Samaniego (1981). Moreover, we use the likelihood ratio and gradient statistics to test hypotheses. We carry out a simulation study to evaluate the performance of the likelihood ratio and gradient tests.

We develop diagnostic tests for the beta regression model with measurement error. We propose weighted standardized residuals as defined by Espinheira et al. (2008b) to verify the assumptions made for the model and to detect outliers. The measures of global influence, such as the generalized Cook's distance and likelihood distance, are used to detect influential points. In addition, we use the conformal approach for evaluating local influence for three perturbation schemes: case-weight perturbation, respose variable perturbation, and perturbation in the covariate with and without measurement error. We apply our results to two sets of real data to illustrate the theory developed. Finally, we present our conclusions and possible future work.

Keywords: Beta regression model, measurement error model, maximum likelihood estimation, pseudo-maximum likelihood estimation, regression calibration, diagnostic analysis. 


\section{Sumário}

Lista de Figuras $\quad$ xiii

Lista de Tabelas $\quad$ Xv

1 Introdução $\quad \mathbf{1}$

1.1 Motivação . . . . . . . . . . . . . . . . . . . . . . . . 6

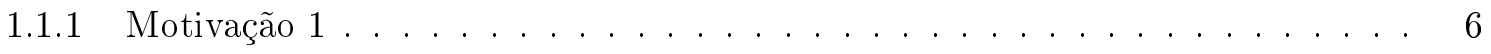

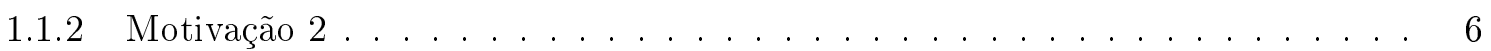

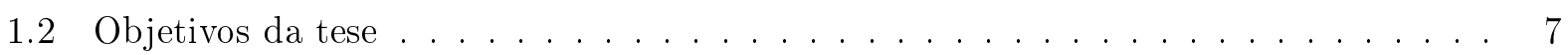

1.3 Organização da tese . . . . . . . . . . . . . . . . . . 8

1.4 Suporte computacional . . . . . . . . . . . . . . . . . . 8

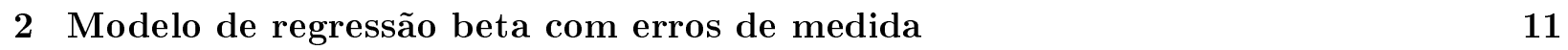

2.1 Modelo de regressão beta com erros de medida . . . . . . . . . . . . . . . . 11

2.2 Estimação . . . . . . . . . . . . . . . . . . . . . . . 13

2.2.1 Estimação por máxima verossimilhança aproximada . . . . . . . . . . 15

2.2.2 Estimação por máxima pseudo-verossimilhança aproximada . . . . . . . . . 16

2.2 .3 Estimação por calibração da regressão . . . . . . . . . . . . . . . . . . 18

2.3 Estudo de simulação . . . . . . . . . . . . . . . . . . . . . . . . 19

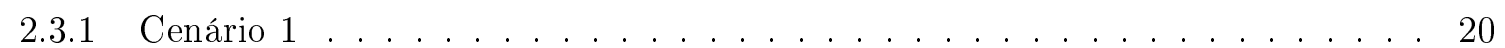

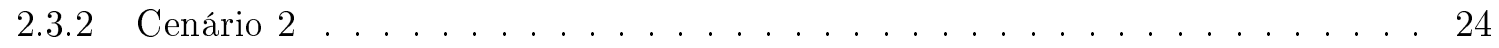

2.3 .3 Cenário $3 \ldots \ldots \ldots \ldots \ldots \ldots \ldots \ldots$

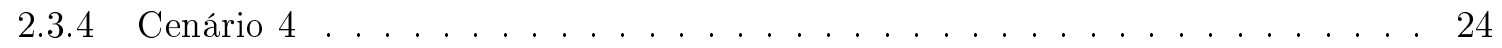

3 Distribuições assintóticas e testes de hipóteses $\quad 35$

3.1 Distribuições assintóticas dos estimadores . . . . . . . . . . . . . . . . . 35

3.1 .1 Máxima verossimilhança aproximada . . . . . . . . . . . . . 35

3.1 .2 Máxima pseudo-verossimilhança aproximada . . . . . . . . . . . 36

3.1 .3 Calibração da regressão . . . . . . . . . . . . . . . . . 38

3.1 .4 Estudo de simulação . . . . . . . . . . . . . . . . . . . . . . 39

3.2 Testes de hipóteses . . . . . . . . . . . . . . . . . . . . . . . 41

3.2 .1 Estudo de simulação . . . . . . . . . . . . . . . . . . . . 43

$\begin{array}{lll}4 & \text { Análise de diagnóstico } & 47\end{array}$

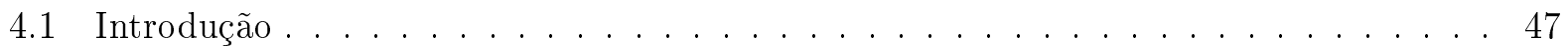

4.2 Análise de Resíduos . . . . . . . . . . . . . . . . . 48

4.2 .1 Resíduos ponderados padronizados . . . . . . . . . . . . . . . 48 
4.2 .2 Envelope simulado . . . . . . . . . . . . . . . . . . 50

4.3 Análise de influência global . . . . . . . . . . . . . . . . . 50

4.4 Análise de influência local . . . . . . . . . . . . . . . . . . 54

4.5 Influência local no modelo de regressão beta com erros de medida . . . . . . . . . . 58

4.5.1 Ponderação de casos . . . . . . . . . . . . . . . . . . 58

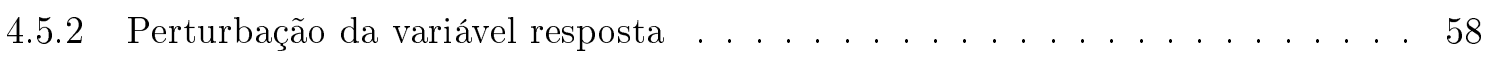

4.5.3 Perturbação de covariáveis . . . . . . . . . . . . . . . . . . . 59

5 Aplicações $\quad 61$

5.1 Aplicação I . . . . . . . . . . . . . . . . . . . . . . . . 61

5.2 Aplicação II . . . . . . . . . . . . . . . . . . . . . . . . . . . . . . 69

6 Considerações finais $\quad \mathbf{8 3}$

6.1 Trabalhos futuros . . . . . . . . . . . . . . . . 83

$\begin{array}{ll}\text { A Apêndice } & \mathbf{8 5}\end{array}$

A.1 Quadratura de Gauss-Hermite . . . . . . . . . . . . . . . . . . . . . . . . 85

A.2 Vetor escore e matriz de informação observada aproximada . . . . . . . . . . . . 87

A.2.1 Vetor escore . . . . . . . . . . . . . . . . . 87

A.2.2 Matriz de informação observada . . . . . . . . . . . . . . . 89

A.3 Vetor escore e matriz de informação observada restrita . . . . . . . . . . . . . 95

A.3.1 Vetor escore . . . . . . . . . . . . . . . . . . 95

A.3.2 Matriz de informação observada . . . . . . . . . . . . . . . . . . . . 96

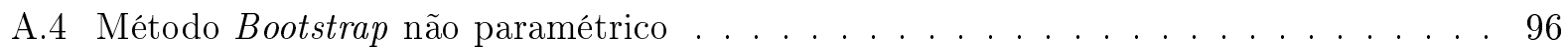

B Apêndice $\quad 99$

B.1 Estimação por máxima verossimilhança aproximada . . . . . . . . . . . . . 99

B.2 Estimação por máxima pseudo-verossimilhança aproximada . . . . . . . . . . . . . 99

B.3 Estimação por calibração da regressão . . . . . . . . . . . . . . . . . . . . 100

B.4 Matriz de variâncias e covariâncias dos estimadores de máxima verossimilhança apro-

ximada . . . . . . . . . . . . . . . . . . . . . . . . . . . .

B.5 Matriz de variâncias e covariâncias dos estimadores de máxima pseudo-verossimilhança aproximada . . . . . . . . . . . . . . . . . . 101

B.6 Matriz de variâncias e covariâncias dos estimadores de calibração da regressão . . . . 103

B.7 Resíduos ponderados padronizados . . . . . . . . . . . . . . . . . . 105

B.8 Análise de influência global . . . . . . . . . . . . . . . . . . . 105

B.9 Análise de influência local . . . . . . . . . . . . . . . . . . 106

B.10 Logaritmo da função de verossimilhança aproximada . . . . . . . . . . . . . . . 107

B.11 Logaritmo da função de verossimilhança restrita . . . . . . . . . . . . . . . . . . . . . . . . . . . . . . . . .

B.12 Logaritmo da função de pseudo-verossimilhança aproximada . . . . . . . . . . . . . 108

B.13 Logaritmo da função de verossimilhança Naïve ou calibração da regressão . . . . . . 109

B.14 Logaritmo da função de pseudo-verossimilhança aproximada: Ponderação de casos . . 110

B.15 Logaritmo da função de pseudo-verossimilhança aproximada: Perturbação da variável

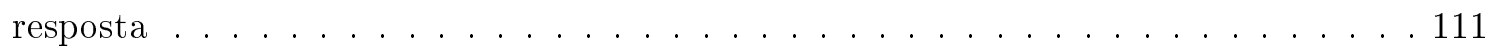


B.16 Logaritmo da função de pseudo-verossimilhança aproximada: Perturbada da variável preditora com erro . . . . . . . . . . . . . . . . . . . . . 111 


\section{Lista de Figuras}

1.1 Gráfico do fator de carga versus $\log ($ consumo $) \ldots \ldots \ldots \ldots \ldots$

4.1 Gráficos normais de probabilidade para os resíduos ponderados padronizados 2 obtidos utilizando o método de máxima verossimilhança para estimar os parâmetros . . . 51

4.2 Gráficos normais de probabilidade para os resíduos ponderados padronizados 2 obtidos utilizando o método de máxima pseudo-verossimilhança aproximada para estimar

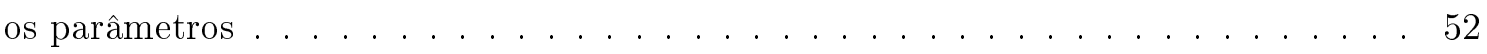

4.3 Gráficos normais de probabilidade para os resíduos ponderados padronizados 2 obtidos utilizando o método de calibração da regressão para estimar os parâmetros . . . 53

5.1 Gráfico do resíduo ponderado padronizado versus (a) índice das observações e (b) valores preditos de $\mathrm{x} \ldots \ldots \ldots \ldots \ldots$. . . . . . . . . . . . . 6 64

5.2 Medidas de influência global, (a) distância de Cook generalizado e (b) afastamento da verossimilhança. . . . . . . . . . . . . . . . . . . . . 65

5.3 (a) Autovalores normalizados em módulo com valores de $q$, (b) influência devida à contribuição agregada do autovetor 1-influente e (c) influência devida à contribuição agregada de todos os autovetores, $m[q]_{j}$ para o esquema de perturbação de casos. . . 66

5.4 (a) Autovalores normalizados em módulo com valores de $q$, (b) influência devida à contribuição agregada do autovetor 1-influente e (c) influência devida à contribuição agregada de todos os autovetores, $m[q]_{j}$ para o esquema de perturbação da variável

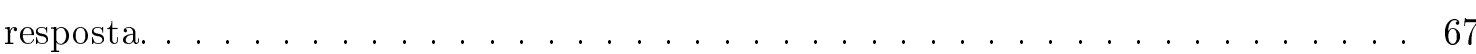

5.5 (a) Autovalores normalizados em módulo com valores de $q$, (b) influência devida à contribuição agregada do autovetor 2-influente e (c) influência devida à contribuição agregada de todos os autovetores, $m[q]_{j}$ para o esquema de perturbação da variável

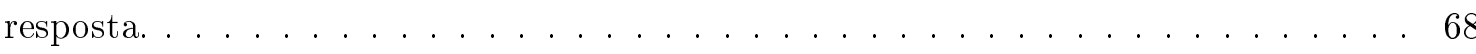

5.6 Gráfico normal de probabilidades com envelope simulado com (a) dados completos, (b) retirando a observação $\sharp 60$ e (c) retirando as observações $\sharp 5$ e $\sharp 60 . \quad \ldots . . .70$

5.7 Gráfico de dispersão do colesterol total (CT) e colesterol ruim (LDL) ambas medições

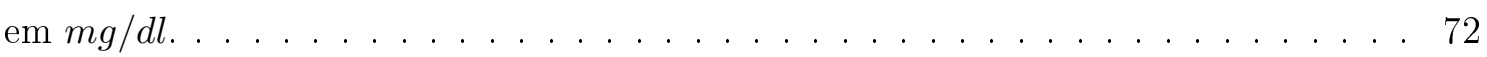

5.8 Gráfico do resíduo ponderado padronizado versus (a) índice das observações e (b)

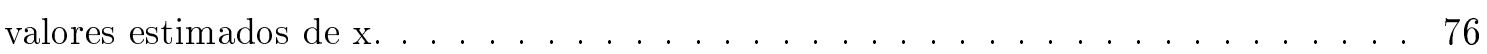

5.9 Medidas de influência global, (a) distância de Cook generalizado e (b) afastamento da verossimilhança. . . . . . . . . . . . . . . . . . . 77 
5.10 (a) Autovalores normalizados em módulo com valores de $q$, (b) influência devida à contribuição agregada do autovetor 1-influente, influência devida à contribuição agregada do autovetor 5-influente (c) e (d) influência devida à contribuição agregada de todos os autovetores, $m[q]_{j}$ para o esquema de perturbação de casos. $\quad$. . . . . . . 78

5.11 (a) Autovalores normalizados em módulo com valores de $q$, (b) influência devida à contribuição agregada do autovetor 1-influente, influência devida à contribuição agregada do autovetor 5-influente (c) e (d) influência devida à contribuição agregada de todos os autovetores, $m[q]_{j}$ para o esquema de perturbação de casos. $\quad$ . . . . . . 79

5.12 (a) Autovalores normalizados em módulo com valores de $q$, (b) influência devida à contribuição agregada do autovetor 1-influente e (c) influência devida à contribuição agregada de todos os autovetores, $m[q]_{j}$ para o esquema de perturbação de casos. . . 80

5.13 Gráficos de envelope simulado para (a) todas as observações e (b) quando retiramos a observação $\sharp 163$ que apresento maior desvio relativo percentual. . . . . . . . . . . . 82

A.1 Aproximação da integral por retângulos de base $c_{i}$ e altura $f\left(x_{i}\right)$. Adaptado da Figura 2 de Peixoto (2008). . . . . . . . . . . . . . . . . . . . 86 


\section{Lista de Tabelas}

2.1 Média, Viés e REQM das estimativas de Monte Carlo dos parâmetros com variância do erro de medida $\sigma_{e}^{2}=0,05$. Verdadeiros valores: $\alpha=2,0, \beta=-0,6, \gamma=2,5, \lambda=0,5$,

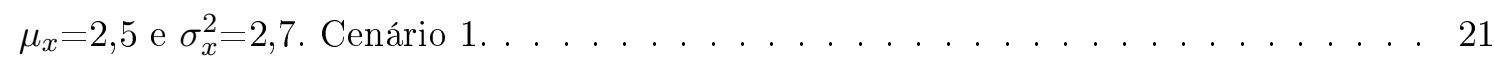

2.2 Média, Viés e REQM das estimativas de Monte Carlo dos parâmetros com variância do erro de medida $\sigma_{e}^{2}=0,50$. Verdadeiros valores: $\alpha=2,0, \beta=-0,6, \gamma=2,5, \lambda=0,5$,

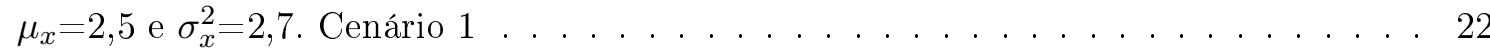

2.3 Média, Viés e REQM das estimativas de Monte Carlo dos parâmetros com variância do erro de medida $\sigma_{e}^{2}=1,0$. Verdadeiros valores: $\alpha=2,0, \beta=-0,6, \gamma=2,5, \lambda=0,5$,

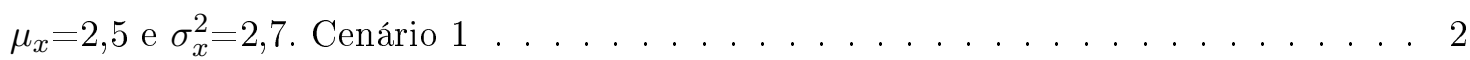

2.4 Média, Viés e REQM das estimativas de Monte Carlo dos parâmetros considerando três réplicas para $w_{t}$. Verdadeiros valores: $\alpha=2,0, \beta=-0,6, \gamma=2,5, \lambda=0,5, \mu_{x}=2,5$,

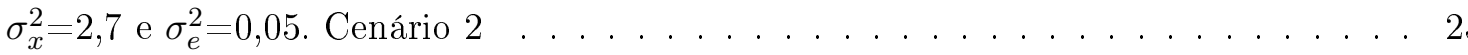

2.5 Média, Viés e REQM das estimativas de Monte Carlo dos parâmetros considerando três réplicas para $w_{t}$. Verdadeiros valores: $\alpha=2,0, \beta=-0,6, \gamma=2,5, \lambda=0,5, \mu_{x}=2,5$,

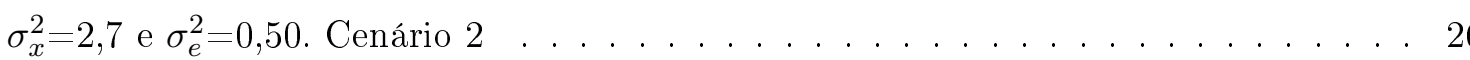

2.6 Média, Viés e REQM das estimativas de Monte Carlo dos parâmetros considerando três réplicas para $w_{t}$. Verdadeiros valores: $\alpha=2,0, \beta=-0,6, \gamma=2,5, \lambda=0,5, \mu_{x}=2,5$,

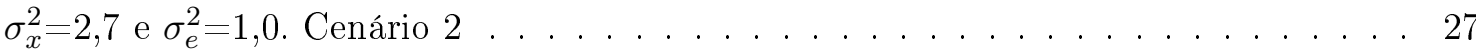

2.7 Média, Viés e REQM das estimativas de Monte Carlo dos parâmetros com variância do erro de medida $\sigma_{e}^{2}=0,05$ e duas covariáveis medidas com erro. Verdadeiros valores: $\alpha=2,0, \beta_{1}=-0,6, \beta_{2}=-1,0, \gamma=2,5, \lambda=0,5, \mu_{x 1}=\mu_{x 2}=2,5$ e $\sigma_{x 1}^{2}=\sigma_{x 2}^{2}=2$,7. Cenário 328

2.8 Média, Viés e REQM das estimativas de Monte Carlo dos parâmetros com variância do erro de medida $\sigma_{e}^{2}=0,50$ e duas covariáveis medidas com erro. Verdadeiros valores: $\alpha=2,0, \beta_{1}=-0,6, \beta_{2}=-1,0, \gamma=2,5, \lambda=0,5, \mu_{x 1}=\mu_{x 2}=2,5$ e $\sigma_{x 1}^{2}=\sigma_{x 2}^{2}=2,7$.

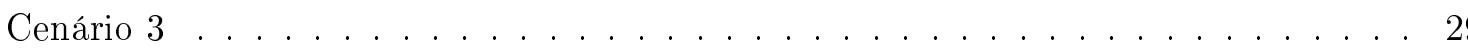

2.9 Média, Viés e REQM das estimativas de Monte Carlo dos parâmetros com variância do erro de medida $\sigma_{e}^{2}=1,0$ e duas covariáveis medidas com erro. Verdadeiros valores: $\alpha=2,0, \beta_{1}=-0,6, \beta_{2}=-1,0, \gamma=2,5, \lambda=0,5, \mu_{x 1}=\mu_{x 2}=2,5$ e $\sigma_{x 1}^{2}=\sigma_{x 2}^{2}=2,7$.

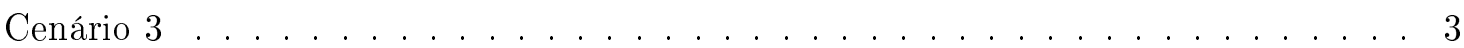

2.10 Média, Viés e REQM das estimativas de Monte Carlo dos parâmetros com variância do erro de medida $\sigma_{e}^{2}=0,05$. Verdadeiros valores: $\alpha=2,0, \beta=-0,6, \gamma=2,5, \lambda=0,5$,

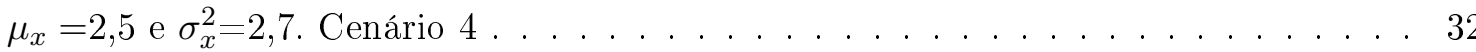

2.11 Média, Viés e REQM das estimativas de Monte Carlo dos parâmetros com variância do erro de medida $\sigma_{e}^{2}=0,5$. Verdadeiros valores: $\alpha=2,0, \beta=-0,6, \gamma=2,5, \lambda=0,5$, $\mu_{x}=2,5$ e $\sigma_{x}^{2}=2,7$. Cenário $4 \ldots \ldots \ldots \ldots \ldots \ldots \ldots$ 
2.12 Média, Viés e REQM das estimativas de Monte Carlo dos parâmetros com variância do erro de medida $\sigma_{e}^{2}=1,0$. Verdadeiros valores: $\alpha=2,0, \beta=-0,6, \gamma=2,5, \lambda=0,5$, $\mu_{x}=2,5$ e $\sigma_{x}^{2}=2,7$. Cenário $4 \ldots \ldots \ldots \ldots \ldots \ldots \ldots$

3.1 Taxas de cobertura dos intervalos de confiança. Método de máxima verossimilhança

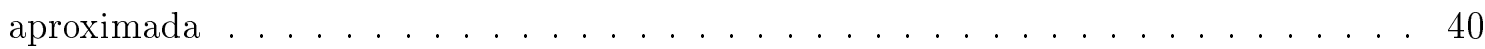

3.2 Taxas de cobertura dos intervalos de confiança. Método de máxima pseudo-verossimilhança

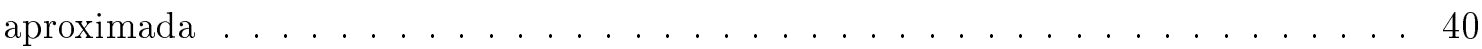

3.3 Taxas de cobertura dos intervalos de confiança. Método de calibração da regressão . 41

3.4 Taxas de cobertura dos intervalos de confiança. Método naïve. . . . . . . . . . . . . 41

3.5 Taxa de rejeição sob $\mathcal{H}_{0}: \beta=0$ dos testes da razão de verossimilhanças e gradiente. Método de máxima verossimilhança aproximada . . . . . . . . . . . . . . . . 44

3.6 Taxa de rejeição sob $\mathcal{H}_{0}: \beta=0$ dos testes da razão de verossimilhanças e gradiente. Método de máxima pseudo-verossimilhança aproximada . . . . . . . . . . . . . 44

3.7 Taxa de rejeição sob $\mathcal{H}_{0}: \beta=0$ dos testes da razão de verossimilhanças e gradiente. Método de calibração da regressão . . . . . . . . . . . . . . . . . . . 44

3.8 Taxa de rejeição sob $\mathcal{H}_{0}: \beta=0$ dos testes da razão de verossimilhanças e gradiente. Método naïve . . . . . . . . . . . . . . . . . . . . 45

3.9 Taxa de rejeição sob $\mathcal{H}_{0}: \lambda=0$ dos testes da razão de verossimilhança e gradiente, no modelo de regressão beta com erros de medida. Método de máxima verossimilhança

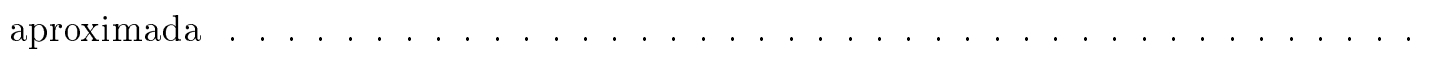

3.10 Taxa de rejeição sob $\mathcal{H}_{0}: \lambda=0$ dos testes da razão de verossimilhança e gradiente, no modelo de regressão beta com erros de medida. Método de máxima pseudoverossimilhança aproximada . . . . . . . . . . . . . . . . .

3.11 Taxa de rejeição sob $\mathcal{H}_{0}: \lambda=0$ dos testes da razão de verossimilhança e gradiente, no modelo de regressão beta com erros de medida. Método de calibração da regressão 46

3.12 Taxa de rejeição sob $\mathcal{H}_{0}: \lambda=0$ dos testes da razão de verossimilhança e gradiente, no modelo de regressão beta com erros de medida. Método naïve . . . . . . . . . 46

5.1 Medidas resumo das variáveis . . . . . . . . . . . . . . . . 61

5.2 Estimativas (E), erros padrão (EP), p-valores (p) e intervalos de confiança (IC) com coeficiente de confiança de $95 \%$ obtidos pelos métodos dados na Seção 2.2 e bootstrap. 62

5.3 Testes de $\mathcal{H}_{0}: \lambda=0$ baseados nas estatísticas da razão de verossimilhanças e gradiente $(\mathrm{p}$-valores entre parênteses) . . . . . . . . . . . . . . . 63

5.4 Estimativas (E), erros padrão (EP), p-valores (p) e intervalos de confiança (IC) com coeficiente de confiança de $95 \%$ obtidos pelos métodos dados na Seção 2.2 e bootstrap do modelo de regressão beta com erros de medida e dispersão constante. . . . . . . . . 63

5.5 Estimativas (E), erros padrão (EP) e desvio relativo percentual (DRP) obtidos pelo método de máxima pseudo-verossimilhança. . . . . . . . . . . . . . . 69

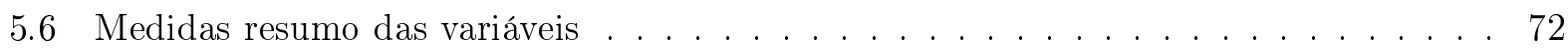

5.7 Estimativas (E), erros padrão (EP), valor-p (p) e intervalo de confiança (IC) obtidos pelos métodos de máxima verossimilhança, pseudo-verossimilhança e bootstrap. . . .

5.8 Testes de $\mathcal{H}_{0}: \gamma_{1}=0$ nas estatísticas da razão de verossimilhanças e gradiente, em parênteses os p-valores. . . . . . . . . . . . . . . . . . . . 
5.9 Estimativas (E), erros padrão (EP), p-valores (p) e intervalo de confiança (IC) obtidos pelos métodos de máxima verossimilhança, pseudo-verossimilhança e bootstrap. . . . 75

5.10 Estimativas (E), erros padrão (EP) e desvio relativo percentual (DRP) obtidos pelo método de máxima pseudo-verossimilhança. . . . . . . . . . . . . . . . 81 


\section{Capítulo 1}

\section{Introdução}

Os modelos de regressão são muito utilizados em diversas áreas do conhecimento, como medicina, economia, engenharia, etc., com o propósito de explicar a variabilidade da variável de interesse (variável resposta) através de uma função das variáveis explicativas (ou covariáveis). Existem diversas classes de modelos de regressão, como por exemplo, lineares ou não lineares, homocedásticos ou heterocedásticos, com ou sem erros nas variáveis. Cada classe de modelos é munida de suposições que devem ser respeitadas a fim de que o processo inferencial seja coerente. Neste trabalho objetivamos estudar uma classe de modelos de regressão não linear em que a variável resposta é contínua e restrita ao intervalo $(0,1)$ e em que uma ou mais variáveis explicativas apresentam erros de medição.

Os modelos com erros nas variáveis são utilizados quando as covariáveis não são observadas diretamente ou estão sujeitas a erros de medição. É importante enfatizar que, na ausência desta suposição, obteremos resultados inferenciais não confiáveis (Rosner e Spiegelman, 1989; Stefanski, 1985; Stefanski e Carroll, 1985). Assim, para compreendermos melhor em quais circunstâncias esta suposição deve ser acrescentada ao modelo, mencionamos a seguir o exemplo 1.2.1 de Fuller (1987). Nesse exemplo, o interesse é avaliar o rendimento da produção de determinado cereal, $y$, em função da porcentagem de nitrogênio no solo, $x$. Foram coletadas 11 diferentes amostras do solo do estado de Iowa nos Estados Unidos e levadas a um laboratório para serem analisadas quimicamente. Apesar dos constantes avanços tecnológicos, não é realista supor que tal análise química não esteja associada a erros de medição.

É comum na prática encontrar esta e outras circunstâncias nas quais os erros de medição podem ser considerados, ver, por exemplo, Fuller (1987), Cheng e Van Ness (1999), Carroll et al. (2006) e Buonaccorsi (2010). Alguns exemplos destas circunstâncias são:

- em métodos e técnicas de coleta de dados, como entrevistas e questionários, entre outros, os erros nas variáveis podem ser ocasionados por desonestidade, confusão, ignorância ou por falta de cuidado;

- erros causados pela incerteza inerente aos equipamentos de medição;

- falhas nos equipamentos ocasionadas por desgaste dos componentes, falta de calibração ou pelas condições ambientais, que geram variabilidade em instrumentos de leitura;

- pela observação indireta de uma variável considerada como substituta;

- processamento inadequado de dados. 
Nestas circunstâncias, os modelos de regressão com erros nas variáveis usualmente são definidos e estruturados de modo que a resposta $y$ seja uma função de preditores ou covariáveis $x$ cujas medições são imprecisas. Assim, no lugar do verdadeiro valor da variável preditora, $x$, considera-se o valor de uma outra variável preditora, $w$, que está associada a um erro de mensuração.

Três formas de modelagem podem ser abordadas para tratar o problema de mensuração de covariáveis com erro. A primeira, em que consideramos uma distribuição de probabilidade para as covariáveis não observadas, é chamada de modelo estrutural. A segunda, em que valores desconhecidos de $x$ são vistos como parâmetros, é denominada de modelo funcional. A terceira, que é uma generalização dos modelos estrutural e funcional, é conhecida como modelo ultraestrutural. No enfoque funcional, o número de parâmetros cresce com o tamanho da amostra. Nesse caso, dizemos que há parâmetros incidentais no modelo. As abordagens estrutural e funcional, como são consideradas em Kendall e Stuart (2009) e Moran (1971), apresentam problemas de identificabilidade e de verossimilhança ilimitada, respectivamente, além de outros problemas teóricos e computacionais; mais detalhes podem ser encontrados em Fuller (1987, Seção 1.1.3). A abordagem ultraestrutural que foi proposta por Dolby (1976) é caracterizada como um modelo estrutural quando as distribuições de probabilidade das covariáveis não observadas apresentam médias iguais aos verdadeiros valores de $x$. Por outro lado, se a variância do verdadeiro valor da covariável é nula, esta abordagem é caracterizada como modelo funcional.

Nessas três abordagens um dos objetivos da análise é encontrar estimadores consistentes para os parâmetros de interesse. Na literatura existe uma grande variedade de técnicas que conduzem a estimadores consistentes em modelos lineares considerando uma abordagem estrutural ou funcional. Algumas envolvem correções explícitas para o vício dos estimadores, enquanto outras propõem estimadores alternativos sob certas suposições como mostram Fuller (1987, Seção 1.0) e Cheng e Van Ness (1999, Seção 4.0). Para o caso dos modelos não lineares, alguns métodos propostos são apropriados somente para estimação no enfoque estrutural, pois requerem conhecimento da distribuição condicional de $x$ dado $w$ (Carroll et al., 2006, p.65). Entre eles podemos citar a estimação pelos métodos de máxima verossimilhança, máxima pseudo-verossimilhança e calibração da regressão. O método de máxima verossimilhança determina as estimativas maximizando diretamente o logaritmo da função de verossimilhança. O método de máxima pseudo-verossimilhança, proposto por Gong e Samaniego (1981), é utilizado quando a inferência em um determinado modelo envolve apenas alguns mas não todos os parâmetros, ou seja, quando o modelo apresenta tanto parâmetros de interesse como também de perturbação. Carroll et al. (1993), por exemplo, estudam os modelos de regressão logística com erros de medida nas variáveis preditoras e usam o método da pseudo-verossimilhança, Guolo (2011) utiliza o algoritmo numérico EM-Monte Carlo para estimar os parâmetros pelo método de máxima pseudo-verossimilhança. Whittemore e Keller (1988) e Armstrong (1985) propõem substituir a verdadeira covariável não observada, $x$, por uma estimativa da esperança condicional de $x$ dado $w, \mathrm{E}(x \mid w)$. Após a substituição, as estimativas dos parâmetros são obtidas por algum método confiável como, por exemplo, por máxima verossimilhança. Podemos citar trabalhos como Carroll et al. (2006), Freedman et al. (2008), Thurston et al. (2005), Guolo (2011), entre outros, que utilizam esta metodologia. Este método é conhecido como calibração da regressão.

É importante comentar que, em modelos com erros nas covariáveis, os estimadores obtidos por algum procedimento usual (mínimos quadrados, máxima verossimilhança, etc.), em que simples- 
mente as verdadeiras covariáveis não observadas são substituídas pelas covariáveis observadas, são chamados estimadores ingênuos (nä̈ve) e são, em geral, inconsistentes (Fuller, 1987, Seção 1.0).

Há vasta literatura acerca de modelos com erros nas variáveis. Entretanto, modelos de regressão com erros nas variáveis para dados contínuos e restritos ao intervalo $(0,1)$ ainda permanecem inexplorados. Esta tese se propõe a completar parte desta lacuna.

Existem varias propostas metodológicas para analisar variáveis dependentes $y$ restritas ao intervalo unitário, isto é $0<y<1$, como proporções. Kieschnick e McCullough (2003) apresentam sete tipos de modelos de regressão que têm sido utilizados para modelar a distribuição de dados de proporção. Segundo os autores, alguns destes modelos violam duas suposições essenciais, a saber:

1. como a variável de interesse é limitada em $(0,1)$, sua função esperança condicional deve ser não linear;

2. as variâncias dos erros aleatórios devem ser diferentes ao longo das observações.

Os sete modelos apresentados em Kieschnick e McCullough (2003) podem ser divididos em duas categorias. Na primeira encontram-se o modelo normal linear, o modelo logito, o modelo normal censurado e o modelo normal não linear. Na segunda categoria estão os modelos de regressão baseados nas distribuições beta e simplex e na metodologia de quase-verossimilhança. Os modelos especificados na primeira categoria caracterizam-se por violar ao menos uma das duas suposições dadas anteriormente. No entanto, os modelos da segunda categoria são aqueles em que nenhuma das suposições é violada.

Uma prática comum para modelar variáveis contínuas no intervalo $(0,1)$ é o uso do modelo normal. Mas, este método, segundo Kieschnick e McCullough (2003), apresenta vários inconvenientes, por exemplo, o fato de proporções não estarem definidas sob o domínio dos números reais, sugerindo que a suposição de normalidade é inadequada.

Transformações na variável dependente são tipicamente utilizados para que $y$ assuma valores na reta, sendo possível modelar a média da variável transformada. Segundo Kieschnick e McCullough (2003), na grande maioria dos trabalhos é utilizada a transformação logito. Essa abordagem apresenta inconvenientes, por exemplo, quando assume-se a transformação logito ou logarítmica, como recomenda Atkinson (1985), espera-se a estabilização da variância, o que pode não ocorrer. Outro inconveniente é que os parâmetros não são interpretáveis em termos da variável resposta original.

No que se refere aos modelos normais censurados, ou modelos tobit, Kieschnick e McCullough (2003) concluem que, para dados contínuos observados no intervalo $(0,1)$, a regressão tobit é observado equivalente à distribuição normal. Portanto, esta abordagem está sujeita às mesmas críticas referentes aos modelos normais lineares ou não lineares.

O modelo de regressão beta proposto por Kieschnick e McCullough (2003) limita-se ao uso da função de ligação logito para a esperança condicional. Outro modelo paramétrico utilizado para modelar proporções no intervalo (0,1), desenvolvido por Barndorff-Nielsen e Jørgensen (1991), é baseado na distribuição simplex.

Kieschnick e McCullough (2003), Papke e Wooldridge (1996) e Cox (1996) discutem o uso da técnica de quase-verossimilhança para a modelagem de proporções. Esta técnica nos permite realizar uma análise inferencial em que não é necessário supor uma distribuição aos dados.

Uma abordagem apropriada utilizada nos últimos anos para modelar variáveis contínuas limitadas ao intervalo $(0,1)$, como proporções medidas de forma contínua, que pode ser explicada por 
outras variáveis através de uma estrutura de regressão, é o modelo de regressão beta proposto por Ferrari e Cribari-Neto (2004). O modelo assume que a variável de interesse, $y$, tem distribuição beta ${ }^{1}$, cuja função de densidade é dada por

$$
\pi(y ; p, q)=\frac{\Gamma(p+q)}{\Gamma(p) \Gamma(q)} y^{p-1}(1-y)^{q-1}
$$

em que $p>0$ e $q>0, \Gamma(\cdot)$ sendo a função gama. A média e a variância de $y$ são, respectivamente, dadas por

$$
\begin{aligned}
\mathrm{E}(y) & =\frac{p}{p+q}, \\
\operatorname{Var}(y) & =\frac{p q}{(p+q)^{2}(p+q+1)} .
\end{aligned}
$$

A definição da estrutura de regressão é feita a partir de uma reparametrização da função densidade dada em (1.1). Sejam $\mu=p /(p+q)$ e $\phi=p+q$ a média e o parâmetro de precisão. Neste caso, temos que $p=\mu \phi$ e $q=(1-\mu) \phi$. A média e a variância de $y$ dadas em (1.2) e (1.3) podem ser escritas como

$$
\begin{aligned}
\mathrm{E}(y) & =\mu, \\
\operatorname{Var}(y) & =\frac{V(\mu)}{1+\phi},
\end{aligned}
$$

em que $V(\mu)=\mu(1-\mu)$ é chamada de função de variância. Reescrevendo (1.1) em termos de $\mu$ e $\phi$, tem-se

$$
f(y ; \mu, \phi)=\frac{\Gamma(\phi)}{\Gamma(\mu \phi) \Gamma[(1-\mu) \phi]} y^{\mu \phi-1}(1-y)^{(1-\mu) \phi-1},
$$

com $0<\mu<1$ e $\phi>0$.

Para uma amostra aleatória $y_{1}, y_{2}, \ldots, y_{n}$ da variável resposta, em que cada $y_{t}, t=1, \ldots, n$, segue a função densidade (1.4) com média $\mu_{t}$ e parâmetro de precisão desconhecido $\phi$, o modelo de regressão beta definido por Ferrari e Cribari-Neto (2004) assume que

$$
g\left(\mu_{t}\right)=\mathbf{z}_{t}^{\top} \boldsymbol{\alpha}
$$

em que $\boldsymbol{\alpha}=\left(\alpha_{1}, \ldots, \alpha_{p}\right)^{\top}$ é um vetor de parâmetros desconhecidos pertencentes ao espaço real $p$ dimensional $\mathbb{R}^{p}$, e $\mathbf{z}_{t}^{\top}=\left(z_{t 1}, \ldots, z_{t p}\right)$ é um vetor de observações fixas e conhecidas de $p$ covariáveis $(p<n)$. A função de ligação $g(\cdot):(0,1) \rightarrow \mathbb{R}$ é, por suposição, estritamente monótona, contínua e duas vezes diferenciável.

Existem várias possibilidades de escolha de $g(\cdot)$, por exemplo, a função de ligação logito $g(\mu)=$ $\log (\mu /(1-\mu))$, a ligação probito $g(\mu)=\Phi^{-1}(\mu)$, em que $\Phi(\cdot)$ é a função de distribuição normal padrão, a ligação complemento $\log -\log g(\mu)=\log \{-\log (1-\mu)\}$, entre outras. Suponhamos por exemplo, que a função de ligação escolhida é logito. Então, $\mu_{t}$ pode ser escrita como

$$
\mu_{t}=\frac{\exp \left(\mathbf{z}_{t}^{\top} \boldsymbol{\alpha}\right)}{1+\exp \left(\mathbf{z}_{t}^{\top} \boldsymbol{\alpha}\right)}, \quad t=1, \ldots, n
$$

\footnotetext{
${ }^{1}$ Mais detalhes sobre a distribuição beta podem ser obtidos ainda em Gupta e Nadarajah (2004).
} 
Supondo que o valor do $p$-ésimo regressor é acrescido de $c$ unidades, mantendo-se as demais covariáveis constantes, a interpretação do parâmetro $\alpha_{p}$ se dá pelo logaritmo da razão de chances

$$
c \alpha_{p}=\log \left(\frac{\mu^{*} /\left(1-\mu^{*}\right)}{\mu /(1-\mu)}\right)
$$

em que $\mu^{*}$ é a média da resposta sob os novos valores das covariáveis.

Extensões do modelo de regressão beta proposto por Ferrari e Cribari-Neto (2004), que permitem que o parâmetro de precisão $\phi$ varie ao longo das observações ou que permitem estruturas não lineares para os parâmetros, são apresentadas por Smithson e Verkuilen (2006) e Simas et al. (2010), entre outros. Um modelo de regressão beta bem geral assume que

$$
\begin{aligned}
& g\left(\mu_{t}\right)=f_{1}\left(\mathbf{z}_{t} ; \boldsymbol{\alpha}\right) \\
& h\left(\phi_{t}\right)=f_{2}\left(\mathbf{v}_{t} ; \boldsymbol{\gamma}\right)
\end{aligned}
$$

em que $f_{1}(\cdot ; \cdot)$ e $f_{2}(\cdot ; \cdot)$ representam funções lineares ou não lineares no segundo argumento, $\boldsymbol{\alpha}$, $\mathbf{z}_{t}^{\top}$ e $g(\cdot)$ foram definidos anteriormente, $\gamma$ é um vetor de parâmetros desconhecidos $\left(\gamma \in \mathbb{R}^{q}\right.$, $p+q<n), \mathbf{v}_{t}=\left(v_{t 1}, v_{t 2}, \cdots, v_{t q}\right)^{\top}$ é um vetor de covariáveis, assumidas também fixas e conhecidas, $h(\cdot):(0, \infty) \longrightarrow \mathbb{R}$ é uma função estritamente monótona e duas vezes diferenciavel. Existem diversas função de ligação para $h(\cdot)$, por exemplo, pode-se utilizar a especificação logarítmica, $h\left(\phi_{t}\right)=\log \left(\phi_{t}\right)$.

Baseado em (1.4), (1.7) e (1.8), $\operatorname{com} f_{1}(\cdot ; \cdot)$ e $f_{2}(\cdot ; \cdot)$ funções identidade por exemplo, segue que o logaritmo da função de verossimilhança é dado por

$$
\ell(\boldsymbol{\alpha}, \boldsymbol{\gamma})=\sum_{t=1}^{n} \ell_{t}\left(\mu_{t}, \phi_{t}\right)
$$

com

$$
\begin{aligned}
\ell_{t}\left(\mu_{t}, \phi_{t}\right)= & \log \Gamma\left(\phi_{t}\right)-\log \Gamma\left(\mu_{t} \phi_{t}\right)-\log \Gamma\left[\left(1-\mu_{t}\right) \phi_{t}\right]+\left(\mu_{t} \phi_{t}-1\right) \log \left(y_{t}\right) \\
& +\left[\left(1-\mu_{t}\right) \phi_{t}\right] \log \left(1-y_{t}\right) .
\end{aligned}
$$

Diferenciando o logaritmo da função de verossimilhança com respeito a $\boldsymbol{\alpha}$ e $\boldsymbol{\gamma}$ obtemos o vetor escore, $\boldsymbol{U}=\left(\boldsymbol{U}_{\boldsymbol{\alpha}}, \boldsymbol{U}_{\boldsymbol{\gamma}}\right)^{\top}$, cujas representações matriciais estão dadas por

$$
\begin{aligned}
\boldsymbol{U}_{\boldsymbol{\alpha}} & =\mathbf{Z}^{\top} \boldsymbol{\Phi} \mathbf{G}\left(\mathbf{y}^{*}-\boldsymbol{\mu}^{*}\right) \\
\boldsymbol{U}_{\boldsymbol{\gamma}} & =\mathbf{V}^{\top} \mathbf{H a}
\end{aligned}
$$

em que $\mathbf{Z}$ é uma matriz $n \times p, \mathbf{z}_{t}^{\top}$ é a $t$-ésima linha dessa matriz, $\mathbf{\Phi}=\operatorname{diag}\left(\phi_{1}, \ldots, \phi_{n}\right), \mathbf{G}=$ $\operatorname{diag}\left\{1 / g_{1}^{\prime}\left(\mu_{1}\right), \ldots, 1 / g_{1}^{\prime}\left(\mu_{n}\right)\right\}, \mathbf{y}^{*}=\left(y_{1}^{*}, \ldots, y_{n}^{*}\right)^{\top} \operatorname{com} y_{t}^{*}=\log \left(y_{t} /\left(1-y_{t}\right)\right), \boldsymbol{\mu}^{*}=\left(\mu_{1}^{*}, \ldots, \mu_{n}^{*}\right)^{\top}$ com $\mu_{t}^{*}=\psi\left(\mu_{t} \phi_{t}\right)-\psi\left[\left(1-\mu_{t}\right) \phi_{t}\right]$, em que $\psi(\cdot)$ é a função digama, $\mathbf{V}$ é uma matriz $n \times q$, cuja $t$-ésima linha é $\mathbf{v}_{t}^{\top}, \mathbf{H}=\operatorname{diag}\left\{1 / h^{\prime}\left(\phi_{1}\right), \ldots, 1 / h^{\prime}\left(\phi_{n}\right)\right\}$ e $\mathbf{a}=\left(a_{1}, \ldots, a_{n}\right)^{\top}$, em que $a_{t}=\mu_{t}\left(y_{t}^{*}-\right.$ $\left.\mu_{t}^{*}\right)+\log \left(1-y_{t}\right)-\psi\left[\left(1-\mu_{t}\right) \phi_{t}\right]+\psi(\phi)$, para todo $t=1, \ldots, n$. Solucionando simultaneamente $U_{\boldsymbol{\alpha}}=0$ e $U_{\boldsymbol{\gamma}}=0$ obtemos os estimadores de máxima verossimilhança de $\boldsymbol{\alpha}$ e $\boldsymbol{\gamma}$. A matriz de informação de Fisher, por sua vez, é o negativo do valor esperado das segundas derivadas do logaritmo da função 
de verossimilhança com relação aos parâmetros, sendo dado por

$$
\boldsymbol{K}=\left[\begin{array}{ll}
\boldsymbol{K}_{\alpha \alpha} & \boldsymbol{K}_{\alpha \gamma} \\
\boldsymbol{K}_{\gamma \alpha} & \boldsymbol{K}_{\gamma \gamma}
\end{array}\right],
$$

em que $\boldsymbol{K}_{\alpha \alpha}=\mathbf{Z}^{\top} \mathbf{\Phi} \mathbf{U Z}, \boldsymbol{K}_{\alpha \gamma}=\boldsymbol{K}_{\gamma \alpha}=\mathbf{Z}^{\top} \mathbf{C G H V}, \boldsymbol{K}_{\gamma \gamma}=\mathbf{V D V}, \operatorname{com} \mathbf{U}=\operatorname{diag}\left\{u_{1}, \ldots, u_{n}\right\}$, $\mathbf{C}=\operatorname{diag}\left\{c_{1}, \ldots, c_{n}\right\}, \mathbf{D}=\operatorname{diag}\left\{d_{1}, \ldots, d_{n}\right\}$, sendo que

$$
\begin{aligned}
u_{t} & =\phi_{t}\left\{\psi^{\prime}\left(\mu_{t} \phi_{t}\right)+\psi^{\prime}\left(\left(1-\mu_{t}\right) \phi_{t}\right)\right\} \frac{1}{\left\{g^{\prime}\left(\mu_{t}\right)\right\}^{2}} \\
c_{t} & =\phi_{t}\left\{\psi^{\prime}\left(\mu_{t} \phi_{t}\right) \mu_{t}-\psi^{\prime}\left[\left(1-\mu_{t}\right) \phi_{t}\right]\left(1-\mu_{t}\right)\right\} \\
d_{t} & =\left[\psi^{\prime}\left(\mu_{t} \phi_{t}\right) \mu_{t}^{2}+\psi^{\prime}\left[\left(1-\mu_{t}\right) \phi_{t}\right]\left(1-\mu_{t}^{2}\right)-\psi^{\prime}\left(\phi_{t}\right)\right] \frac{1}{\left\{h^{\prime}\left(\phi_{t}\right)\right\}^{2}}
\end{aligned}
$$

Sob condições de regularidade (Cox e Hinkley, 1974, Pag. 107), os estimadores de máxima verossimilhança $\widehat{\boldsymbol{\alpha}}$ e $\widehat{\boldsymbol{\gamma}}$ de $\boldsymbol{\alpha}$ e $\boldsymbol{\gamma}$ têm distribuição normal com médias $\boldsymbol{\alpha}$ e $\boldsymbol{\gamma}$ e matriz de variâncias e covariâncias $\boldsymbol{K}^{-1}$.

O objetivo central desta tese é estudar a classe de modelos de regressão beta definida acima assumindo que uma ou mais variáveis explicativas apresentam erros de medição.

\subsection{Motivação}

O interesse em estudar uma classe de modelos de regressão beta com erros de medida foi motivada por alguns casos práticos que são descritos a seguir.

\subsubsection{Motivação 1}

A Companhia de Gás de São Paulo (Comgás) é uma empresa brasileira, considerada pela ABEGAS, Associação Brasileira das Empresas Distribuidoras de Gás Canalizado, como a maior distribuidora de gás natural do Brasil. A Comgás distribui gás canalizado para mais de 1 milhão de consumidores nos segmentos residencial, comercial e industrial. Em 2004 a Comgás teve como objetivo determinar um novo modelo tarifário. Deste modo solicitou ao Centro de Metrologia de Fluidos do Instituto de Pesquisa Tecnológica (IPT) um estudo de caracterização de carga (demanda de gás em $m^{3} / h$ ) em cada segmento de mercado. A caracterização de carga do sistema Comgás é finalizada com o cálculo do fator de carga (FC) dos segmentos. O fator de carga representa o quociente entre a demanda média de um determinado segmento e a demanda máxima num período de tempo. Neste problema, a variável resposta, y, é o fator de carga, que pertence ao intervalo $(0,1)$, e a covariável observada, $w$, é o logaritmo do consumo médio mensal em $\left(\mathrm{m}^{3} / h\right)$. Nosso interesse será relacionar a variável resposta, $y$, com a covariável $x$, no segmento residencial. Assumimos que o logaritmo do consumo médio mensal, w, é uma covariável medida com erro. Podemos observar na Figura 1.1 o gráfico de dispersão do fator de carga versus o logaritmo dos consumos médios mensais para os dados do segmento residencial, sugerindo um comportamento não linear. Assim, neste problema prático vemos por conveniente o uso do modelo de regressão beta com erros de medida na covariável.

\subsubsection{Motivação 2}

Trabalhos na área médica foram a motivação para vários autores estudarem modelos com erros de medida, por exemplo, Carroll et al. (2006) e Buonaccorsi (2010). Nós também fomos motivados por um estudo médico, que foi desenvolvido pelo Departamento de Biometria da Universidade 


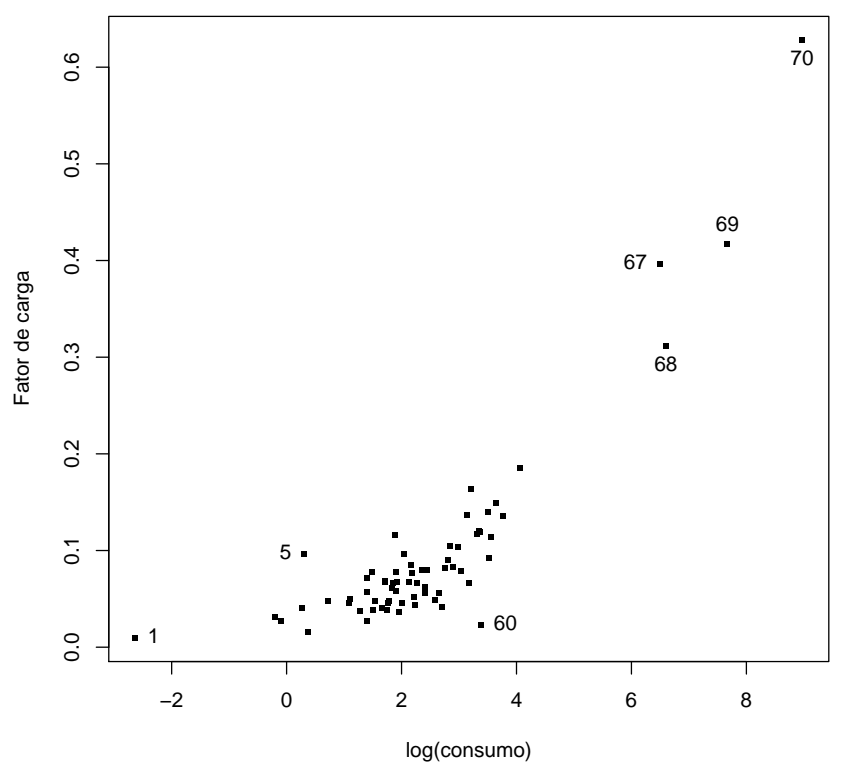

Figura 1.1: Gráfico do fator de carga versus $\log$ (consumo)

Estadual Paulista "Júlio de Mesquita Filho", cujo objetivo foi encontrar fatores de risco cardíaco em 182 mulheres fumantes com idades entre 50 e 87 anos $^{2}$. As variáveis pressão arterial sistólica (PAS), pressão arterial diastólica (PAD), índice de massa corporal (IMC), nível de colesterol total e nível de colesterol "ruim"(LDL), foram consideradas. A diferença de PAS e PAD resulta a pressão do pulso, $P P=P A S-P A D$. Por exemplo, se a pressão arterial for 12 por 8 milímetros de mercúrio (mmHg) a pressão de pulso será $4 \mathrm{mmHg}$, que é considerada uma pressão de pulso normal e saudável (Benetos et al., 2010). Uma PP elevada pode ser um forte indicador de problemas cardíacos. Para nossa análise, uma variável contínua, $y$, limitada ao intervalo $(0,1)$ é construída a partir de PAS e PAD como $y=(P A S-P A D) / P A S=P P / P A S$, que mede a diferença relativa entre as pressões sistólica e diastólica. É considerado que se $y$ for inferior ao 25\%, o indivíduo apresenta problemas cardíacos congênitos (American College of Surgeons, 2008, p. 58). Nesta aplicação o objetivo é estudar o comportamento de $y$ usando as variáveis IMC e LDL. No entanto, LDL é medido com alto custo e tempo, requerendo equipamentos especializados. Aqui utilizamos a variável colesterol total (CT) como uma variável substituta de LDL. Consideramos adequado utilizar o modelo de regressão beta com erros de medida a este conjunto de dados.

\subsection{Objetivos da tese}

A presente tese de doutorado tem como objetivo o estudo dos modelos de regressão beta na presença de erros de medida sob o enfoque estrutural. Serão estudados os seguintes métodos de estimação: máxima verossimilhança, máxima pseudo-verossimilhança e calibração da regressão. Com o intuito de avaliar o comportamento dos estimadores, será apresentado um estudo de simulação via Monte Carlo. Objetivamos também, encontrar a distribuição assintótica dos estimadores da forma como sugerem Gong e Samaniego (1981), Carroll et al. (2006, Seção A.6.6) e Guolo (2011), obter intervalos de confiança para os parâmetros e testar hipóteses. Para possibilitar diagnóstico da qualidade de ajuste do modelo, será proposta uma análise envolvendo resíduos e medidas de

\footnotetext{
${ }^{2}$ Não temos autorização para divulgar o conjunto de dados.
} 
influência global, local e total. Finalmente, os resultados obtidos serão aplicados a conjuntos de dados reais.

\subsection{Organização da tese}

Esta tese encontra-se dividida em seis capítulos. No segundo capítulo propomos o modelo de regressão beta na presença de erros de medida sob o enfoque estrutural. Desenvolvemos inferência baseada nos métodos de máxima verossimilhança, máxima pseudo-verossimilhança e calibração da regressão. Apresentamos expressões para o vetor escore e a matriz de informação observada. Realizamos um estudo de simulação para avaliar o comportamento dos estimadores. No Capítulo 3 encontramos a distribuição assintótica dos estimadores dos parâmetros do modelo de regressão beta com erros de medida. Realizamos testes de hipóteses e um estudo de simulação para avaliar a taxa de rejeição dos testes da razão de verossimilhança e gradiente.

No quarto capítulo tratamos do problema de diagnóstico para o modelo proposto no Capítulo 2. Derivamos resíduos, tornando possível a construção de bandas de confiança (envelopes simulados) para a detecção de possíveis afastamentos das suposições do modelo. Realizamos um estudo de simulação com o objetivo de conhecer a distribuição empírica dos resíduos. Em seguida, desenvolvemos metodologias de diagnóstico baseadas em análise de influência global, influência local e total via curvatura normal proposta por Cook (1986) e Poon e Poon (1999). Consideramos três esquemas de perturbação, a saber: ponderação de casos, perturbação da variável resposta e perturbação das covariáveis medidas com e sem erros de medida.

No quinto capítulo aplicamos os resultados aos conjuntos de dados descritos nas Seções 1.1.1 e 1.1.2. No Capítulo 6 apresentamos nossas conclusões e indicamos direções para novas pesquisas.

Em cada capítulo da tese é apresentado o suporte computacional para exemplificar numericamente a teoria desenvolvida. Mais detalhes sobre as ferramentas computacionais utilizada nesta tese são apresentados na seção seguinte.

\subsection{Suporte computacional}

A linguagem de programação ox (versão 6.2), criada por Jurgen Doornik, constitui a plataforma computacional utilizada no desenvolvimento da tese. Esta linguagem de programação foi desenvolvida por Jurgen Doornik e está disponível gratuitamente para uso acadêmico em http: //www.doornik.com. Essa linguagem permite a implementação de técnicas estatísticas com facilidade e atende a requisitos como precisão e eficiência, o que tem contribuído para sua ampla utilização no campo da computação numérica. Detalhes sobre tal linguagem de programação podem ser encontrados em Doornik (2011). Utilizamos também a linguagem de programação R (versão 2.13.1) com o ambiente TinnR (versão 2.3.7.1) para a realização de gráficos. R é um sistema integrado para computação estatística e geração de gráficos e encontra-se disponível gratuitamente em http://www.r-project.org; Murrell (2006) é uma boa referência. Ademais, este trabalho foi digitado no sistema tipográfico $\mathrm{LAT}_{\mathrm{E}} \mathrm{X}$, que consiste em uma série de macros e rotinas baseadas no sistema $\mathrm{T}_{\mathrm{E}} \mathrm{X}$. Em particular, esta tese foi tipografada nos padrões exigidos pelo Instituto de Matemática e Estatística da Universidade de São Paulo. Os arquivos com extensão .tex podem ser encontrados em http://www.vision.ime.usp.br/ ${ }^{\text {jmena/stuff/tese-exemplo/. }}$

Para o estudo de simulação utilizamos o cluster Puma, http://www.usp.br/lcca/tdi.html, administrado pelo Laboratório de Computação Científica Avançada (LCCA) da Universidade de São Paulo, constituído por 59 servidores DELL PowerEdge 1950, con dois processadores Xeon 5430 (8 
núcleos de 2,66 GHz), 16 GB de RAM DDR2-FBDIMM 667 MHz e com 300 GB de disco SAS cada. 


\section{Capítulo 2}

\section{Modelo de regressão beta com erros de medida}

A ideia central deste capítulo é introduzir os modelos de regressão beta em que uma ou mais variáveis preditoras são medidas com erros. Apresentamos e discutimos técnicas de estimação como máxima verossimilhança, pseudo-verossimilhança e calibração da regressão. Realizamos um estudo de simulação de Monte Carlo com o objetivo de avaliar o comportamento dos estimadores.

\subsection{Modelo de regressão beta com erros de medida}

Diferentes versões de modelos de regressão beta foram definidos por Ferrari e Cribari-Neto (2004), Smithson e Verkuilen (2006) e Simas et al. (2010). Aqui assumimos que $y_{1}, \ldots, y_{n}$ são variáveis aleatórias independentes tais que, para cada $t=1, \ldots, n, y_{t}$ tem distribuição beta com função densidade da forma

$$
f\left(y_{t} ; \mu_{t}, \phi_{t}\right)=\frac{\Gamma\left(\phi_{t}\right)}{\Gamma\left(\mu_{t} \phi_{t}\right) \Gamma\left[\left(1-\mu_{t}\right) \phi_{t}\right]} y_{t}^{\mu_{t} \phi_{t}-1}\left(1-y_{t}\right)^{\left(1-\mu_{t}\right) \phi_{t}-1}, \quad 0<y_{t}<1,
$$

em que $0<\mu_{t}<1$ e $\phi_{t}>0$. Como mencionado anteriormente, $\mathrm{E}\left(y_{t}\right)=\mu_{t}$ e $\operatorname{Var}\left(y_{t}\right)=\mu_{t}(1-$ $\left.\mu_{t}\right) /\left(1+\phi_{t}\right)$. Notemos que, uma vez fixada a média da variável resposta, $\mu_{t}$, quanto maior for o valor de $\phi_{t}$, menor será a variância de $y_{t}$. Assim, $\phi_{t}$ pode ser interpretado como um parâmetro de precisão. O modelo de regressão beta que consideramos aqui é dado pela função densidade (2.1) com as expressões em (1.7) e (1.8), dadas por

$$
\begin{aligned}
& g\left(\mu_{t}\right)=\mathbf{z}_{t}^{\top} \boldsymbol{\alpha}, \\
& h\left(\phi_{t}\right)=\mathbf{v}_{t}^{\top} \boldsymbol{\gamma},
\end{aligned}
$$

em que $\boldsymbol{\alpha} \in \mathbb{R}^{p}, \boldsymbol{\gamma} \in \mathbb{R}^{\breve{q}}, \mathbf{z}_{t}^{\top}=\left(z_{t 1}, \ldots, z_{t p}\right)$ e $\mathbf{v}_{t}^{\top}=\left(v_{t 1}, \ldots, v_{t \breve{q}}\right)$ são vetores de observações fixas e conhecidas de $p$ e $q$ covariáveis, $(p+\breve{q}<n)$.

$\mathrm{Na}$ prática é comum que algumas variáveis explicativas dos vetores $\mathbf{z}_{t}$ ou $\mathbf{v}_{t}$ em (2.2) e (2.3) não sejam observadas diretamente, mas sim obtidas com possíveis erros de medição. Neste caso, os resultados inferenciais não serão confiáveis se a suposição dos erros de medição não for incorporada ao modelo (Rosner e Spiegelman, 1989; Stefanski, 1985; Stefanski e Carroll, 1985). Neste trabalho estudaremos procedimentos inferenciais para o modelo de regressão beta em que uma ou mais variáveis preditoras são medidas com erros.

O modelo de regressão beta com erros de medida considerado aqui é dado por (2.1) com as 
equações (2.2) e (2.3) substituídas por

$$
\begin{aligned}
& g\left(\mu_{t}\right)=\mathbf{z}_{t}^{\top} \boldsymbol{\alpha}+\mathbf{x}_{t}^{\top} \boldsymbol{\beta}, \\
& h\left(\phi_{t}\right)=\mathbf{v}_{t}^{\top} \boldsymbol{\gamma}+\mathbf{m}_{t}^{\top} \boldsymbol{\lambda},
\end{aligned}
$$

em que $\boldsymbol{\beta} \in \mathbb{R}^{r}, \boldsymbol{\lambda} \in \mathbb{R}^{s}$ são vetores de parâmetros desconhecidos, $(p+r)+(\breve{q}+s)<n$, $\mathbf{x}_{t}=\left(x_{t 1}, x_{t 2}, \cdots, x_{t r}\right)^{\top}$ e $\mathbf{m}_{t}=\left(m_{t 1}, m_{t 2}, \cdots, m_{t s}\right)^{\top}$ são vetores de covariáveis não observados diretamente ou associados a erros de medição, podendo ser considerados como variáveis latentes no enfoque estrutural ou como parâmetros incidentais no enfoque funcional. Ambos os modelos, estrutural e funcional, devem ser tratados cuidadosamente devido aos problemas de identificabilidade e verossimilhança ilimitada, respectivamente. Usualmente, tais problemas são contornados assumindo que alguns parâmetros ou alguma relação entre eles é conhecida (Cheng e Van Ness, 1999; Fuller, 1987).

O foco deste trabalho está restrito a estudar o modelo de regressão beta com erros de medida sob o enfoque estrutural, deixando como linha de pesquisa futura o caso funcional.

Os vetores de variáveis preditoras medidas sem erro, $\mathbf{z}_{t}$ e $\mathbf{v}_{t}$, podem ou não conter variáveis em comum. O mesmo ocorre para as variáveis medidas com erros, $\mathbf{x}_{t}$ e $\mathbf{m}_{t}$. Entretanto, para facilitar a descrição das metodologias que serão apresentadas vamos considerar que $\mathbf{x}_{t}$ e $\mathbf{m}_{t}$ coincidem, ou seja, as mesmas variáveis medidas com erros são usadas na especificação da média e da precisão da variável resposta. Note-se que as metodologias abordadas neste trabalho podem ser facilmente estendidas para a situação em que $\mathbf{x}_{t}$ e $\mathbf{m}_{t}$ são distintos, ou mesmo em que há variáveis medidas com erros apenas na especificação da média ou da precisão da variável resposta.

No modelo de regressão beta com erros de medida definido acima, o vetor de variáveis aleatórias $\mathbf{x}_{t}$ não é observado diretamente, ou seja observamos $\mathbf{w}_{t}=\left(w_{t 1}, \ldots, w_{t r}\right)^{\top}$, assumindo ter a seguinte relação com $\mathbf{x}_{t}$ :

$$
\mathbf{w}_{t}=\Im_{1}\left(\mathbf{x}_{t} ; \boldsymbol{\tau}\right)+\mathbf{e}_{t},
$$

em que $\Im_{1}(\cdot ; \boldsymbol{\tau})$ representa uma função linear ou não linear do vetor de parâmetros $\boldsymbol{\tau}$ e $\mathbf{e}_{t}=$ $\left(e_{t 1}, e_{t 2}, \ldots, e_{t r}\right)^{\top}$ é um vetor de erros aleatórios. Um caso particular de (2.6) é dado por

$$
\mathbf{w}_{t}=\tau_{0}+\tau_{1} \circ \mathbf{x}_{t}+\mathbf{e}_{t}
$$

em que $\boldsymbol{\tau}_{0}=\left(\tau_{01}, \tau_{02}, \ldots, \tau_{0 r}\right)^{\top}$ e $\boldsymbol{\tau}_{1}=\left(\tau_{11}, \tau_{12}, \ldots, \tau_{1 r}\right)^{\top}$ são vetores de parâmetros desconhecidos $\left(\boldsymbol{\tau}_{0}, \boldsymbol{\tau}_{1} \in \mathbb{R}^{r}\right)$ e o representa o produto de Hadamard (elemento a elemento). Considerando que $\boldsymbol{\tau}_{0}$ é um vetor de zeros e $\boldsymbol{\tau}_{1}$ é um vetor de uns, temos que a equação anterior se reduz ao modelo clássico

$$
\mathbf{w}_{t}=\mathbf{x}_{t}+\mathbf{e}_{t} .
$$

Sob o enfoque estrutural, assumimos neste trabalho que a distribuição do vetor da variável não observada, $\mathbf{x}_{t}$, é normal. Especificamente, admitimos que $x_{t i} \sim N\left(\mu_{x i}, \sigma_{x i}^{2}\right)$, com $\mu_{x i} \in \mathbb{R}$ e $\sigma_{x i}^{2}>0$ representando a média e a variância da $i$-ésima variável preditora não observável, e são independentes. Supomos também que os erros aleatórios são independentes e $e_{t i} \sim N\left(0, \sigma_{e i}^{2}\right)$. Finalmente, assumimos independência entre $x_{t i}$ e $e_{t^{\prime} i^{\prime}}$, como $t, t^{\prime}=1,2, \ldots, n$ e $i, i^{\prime}=1,2, \ldots, r$.

Como comentado anteriormente, os modelos com erros de medida sob o enfoque estrutural devem 
ser tratados levando-se em conta o problema de identificabilidade. Para contornar tal problema é usual assumir conhecida alguma quantidade, por exemplo, as variâncias dos erros de medida, ou uma das razões $\sigma_{x i}^{2} / \sigma_{e i}^{2}$ ou $\sigma_{x i}^{2} /\left(\sigma_{x i}^{2}+\sigma_{e i}^{2}\right)$. Pode-se estimar em particular, as variâncias dos erros de medida e considerá-as conhecidas, sempre e quando há $n_{t i}$ réplicas para $w_{t i}, i=1,2, \ldots, r$ (Carroll et al., 2006, Cap. 4). Sob essa suposição, o modelo com erros de medida (2.7) tem a seguinte estrutura

$$
\mathbf{w}_{t i j}=\mathbf{x}_{t i}+\mathbf{e}_{t i}, \quad t=1,2, \ldots, n, \quad i=1, \ldots, r, \quad j=1,2, \ldots, n_{t i},
$$

em que $\mathbf{w}_{t i j}$ é a $j$-ésima observação da $i$-ésima covariável medida com erros para o indivíduo $t$. Na presença de réplicas, o desvio das observações em torno da média representa a variabilidade intrapessoal para cada t-ésimo indivíduo. Sendo assim, as variâncias dos erros de medida podem ser estimadas por

$$
\widehat{\sigma}_{e i}^{2}=\frac{1}{\sum_{t=1}^{n}\left(n_{t i}-1\right)} \sum_{t=1}^{n} \sum_{j=1}^{n_{t i}}\left(w_{t i j}-\bar{w}_{t i .}\right)^{2}
$$

em que

$$
\bar{w}_{t i .}=\frac{1}{n_{t i}} \sum_{j=1}^{n_{t i}} w_{t i j} .
$$

Uma propriedade importante deste estimador é que $\mathrm{E}\left[\widehat{\sigma}_{e i}^{2}\right]=\sigma_{e i}^{2}$, ou seja, o estimador de $\sigma_{e i}^{2}$ é não viesado.

Em resumo, o modelo a ser considerado neste trabalho é definido pelas variáveis aleatórias $y_{1}, y_{2}, \ldots, y_{n}$ independentes com função distribuição de probabilidade (2.1) em que $\mu_{t}$ e $\phi_{t}$ obedecem às relações (2.4) e (2.5). Para efeito da descrição das metodologias de inferência admitiremos que as variáveis observadas com erros na especificação da média e da precisão são as mesmas. Para cada $t, t=1,2, \ldots, n$, observa-se o par $\left(y_{t}, \mathbf{w}_{t}\right)$ além das covariáveis medidas sem erros $\mathbf{z}_{t}$ e $\mathbf{v}_{t}$, assumidas fixas e conhecidas. As variáveis aleatórias $x_{t 1}, x_{t 2}, \ldots, x_{t r}$, são não observáveis; em seu lugar observam-se $w_{t 1}, w_{t 2}, \ldots, w_{t r}$ com estrutura dada por (2.7), e as suposições dadas acima. Se há réplicas para as observações com erros de $x_{t}$, assumimos a estrutura dada em (2.8).

Com o intuito de realizar inferência apresentamos a seguir algumas técnicas de estimação dos parâmetros do modelo de regressão beta com erros de medida.

\subsection{Estimação}

Nesta seção abordaremos a estimação dos parâmetros por três métodos. O primeiro é o método de máxima verossimilhança, em que as estimativas dos parâmetros são obtidas a partir da maximização direta do logaritmo da função de verossimilhança. O segundo, por sua vez, é o método da máxima pseudo-verossimilhança, em que os parâmetros de perturbação são estimados previamente a partir de alguma metodologia usual, por exemplo, a de máxima verossimilhança, para depois serem substituídos no logaritmo da função de verossimilhança original. O terceiro método é o de calibração da regressão, em que os estimadores são obtidos fazendo a troca das covariáveis não observadas $\mathbf{x}_{t}$ por uma estimativa do valor esperado da distribuição condicional de $\mathbf{x}_{t}$ dado $\mathbf{w}_{t}$.

Considerando um conjunto finito de pares $\left(y_{1}, \mathbf{w}_{1}\right),\left(y_{2}, \mathbf{w}_{2}\right), \ldots,\left(y_{n}, \mathbf{w}_{n}\right)$ de variáveis observadas. Omitiremos na notação os vetores das variáveis observadas $\mathbf{z}_{t}$ e $\mathbf{v}_{t}$ dado que são fixas e 
conhecidas. Assim, podemos obter a função densidade conjunta de $\left(y_{t}, \mathbf{w}_{t}\right)$, que é o par de observações para o t-ésimo indivíduo, integrando a função densidade conjunta dos dados completos $\left(y_{t}, \mathbf{w}_{t}, \mathbf{x}_{t}\right)$, denotada por $f\left(y_{t}, \mathbf{w}_{t}, \mathbf{x}_{t} ; \mathbf{\Psi}\right)$, com respeito a $\mathbf{x}_{t}$. Omitimos na notação os vetores $\mathbf{z}_{t}$ e $\mathbf{v}_{t}$ dado que estas são consideradas variáveis conhecidas, fixas e livres de erros de medida. Aqui $\boldsymbol{\Psi}=\left(\boldsymbol{\theta}^{\top}, \boldsymbol{\xi}^{\top}, \boldsymbol{\sigma}_{e}^{2}\right)^{\top}$ é o vetor de parâmetros desconhecidos sendo que $\boldsymbol{\theta}=\left(\boldsymbol{\alpha}^{\top}, \boldsymbol{\beta}^{\top}, \boldsymbol{\gamma}^{\top}, \boldsymbol{\lambda}^{\top}\right)^{\top}$ representa o vetor de parâmetros de interesse e $\left(\boldsymbol{\xi}^{\top}, \boldsymbol{\sigma}_{e}^{2 \top}\right)^{\top}=\left(\boldsymbol{\mu}_{x}^{\top}, \boldsymbol{\sigma}_{x}^{2 \top}, \boldsymbol{\sigma}_{e}^{2 \top}\right)^{\top}$, o de parâmetros de perturbação. Aqui, $\boldsymbol{\mu}_{x}=\left(\mu_{x 1}, \mu_{x 2}, \ldots, \mu_{x r}\right)^{\top}, \boldsymbol{\sigma}_{x}^{2}=\left(\sigma_{x 1}^{2}, \sigma_{x 2}^{2}, \ldots, \sigma_{x r}^{2}\right)^{\top}$ e $\boldsymbol{\sigma}_{e}^{2}=\left(\sigma_{e 1}^{2}, \sigma_{e 2}^{2}, \ldots, \sigma_{e r}^{2}\right)^{\top}$. Assim, a função densidade conjunta de $\left(y_{t}, \mathbf{w}_{t}\right)$ é dada por

$$
\begin{aligned}
f\left(y_{t}, \mathbf{w}_{t} ; \boldsymbol{\Psi}\right) & =\int_{-\infty}^{\infty} \cdots \int_{-\infty}^{\infty} f\left(y_{t}, \mathbf{w}_{t}, \mathbf{x}_{t} ; \boldsymbol{\Psi}\right) d \mathbf{x}_{t} \\
& =\int_{-\infty}^{\infty} \cdots \int_{-\infty}^{\infty} f\left(y_{t} \mid \mathbf{w}_{t}, \mathbf{x}_{t} ; \boldsymbol{\theta}\right) f\left(\mathbf{w}_{t} \mid \mathbf{x}_{t} ; \boldsymbol{\sigma}_{e}^{2}\right) f\left(\mathbf{x}_{t} ; \boldsymbol{\xi}\right) d \mathbf{x}_{t} \\
& =\int_{-\infty}^{\infty} \cdots \int_{-\infty}^{\infty} f\left(y_{t} \mid \mathbf{x}_{t} ; \boldsymbol{\theta}\right) f\left(\mathbf{w}_{t} \mid \mathbf{x}_{t} ; \boldsymbol{\sigma}_{e}^{2}\right) f\left(\mathbf{x}_{t} ; \boldsymbol{\xi}\right) d \mathbf{x}_{t}
\end{aligned}
$$

em que $f\left(y_{t} \mid \mathbf{x}_{t} ; \boldsymbol{\theta}\right)$ é a função densidade da distribuição beta dada em (2.1), $f\left(\mathbf{w}_{t} \mid \mathbf{x}_{t} ; \boldsymbol{\sigma}_{e}^{2}\right)$ é a função densidade condicional de $\mathbf{w}_{t}$ dado $\mathbf{x}_{t}$, ou seja, a função densidade da distribuição $N_{r}\left(\mathbf{x}_{t}, \operatorname{diag}\left(\sigma_{e 1}^{2}\right.\right.$, $\left.\left.\sigma_{e 2}^{2}, \ldots, \sigma_{e r}^{2}\right)\right)$ e $f\left(\mathbf{x}_{t} ; \boldsymbol{\xi}\right)$ é a função densidade da variável explicativa $\mathbf{x}_{t}$, cuja distribuição é $N_{r}\left(\boldsymbol{\mu}_{x}\right.$, $\left.\operatorname{diag}\left(\sigma_{x 1}^{2}, \sigma_{x 2}^{2}, \ldots, \sigma_{x r}^{2}\right)\right)$. Note que, para a obtenção de $(2.9)$, assumimos que o erro de medida é não diferenciável (Carroll et al., 2006, p.36), isto é, que a distribuição de $y_{t}$ dado $\left(\mathbf{w}_{t}, \mathbf{x}_{t}\right)$ depende apenas de $\mathbf{x}_{t}$.

O logaritmo da função de verossimilhança das $n$ observações independentes correspondente à função de densidade conjunta (2.9) da amostra observada é dado por

$$
\begin{aligned}
\ell(\boldsymbol{\Psi}) & =\sum_{t=1}^{n} \log f\left(y_{t}, \mathbf{w}_{t} ; \mathbf{\Psi}\right) \\
& =\sum_{t=1}^{n} \log \int_{-\infty}^{\infty} \cdots \int_{-\infty}^{\infty} f\left(y_{t} \mid \mathbf{x}_{t} ; \boldsymbol{\theta}\right) f\left(\mathbf{w}_{t} \mid \mathbf{x}_{t} ; \boldsymbol{\sigma}_{e}^{2}\right) f\left(\mathbf{x}_{t} ; \boldsymbol{\xi}\right) d \mathbf{x}_{t}
\end{aligned}
$$

ou, equivalentemente,

$$
\begin{aligned}
\ell(\mathbf{\Psi}) & =\sum_{t=1}^{n} \log \int_{-\infty}^{\infty} \cdots \int_{-\infty}^{\infty} f\left(y_{t} \mid \mathbf{x}_{t} ; \boldsymbol{\theta}\right) f\left(\mathbf{x}_{t} \mid \mathbf{w}_{t} ; \boldsymbol{\xi}, \sigma_{e}^{2}\right) f\left(\mathbf{w}_{t} ; \boldsymbol{\xi}, \boldsymbol{\sigma}_{e}^{2}\right) d \mathbf{x}_{t}, \\
& =\sum_{t=1}^{n} \log f\left(\mathbf{w}_{t} ; \boldsymbol{\xi}, \boldsymbol{\sigma}_{e}^{2}\right)+\sum_{i=1}^{n} \log \int_{-\infty}^{\infty} \cdots \int_{-\infty}^{\infty} f\left(y_{t} \mid \mathbf{x}_{t} ; \boldsymbol{\theta}\right) f\left(\mathbf{x}_{t} \mid \mathbf{w}_{t} ; \boldsymbol{\xi}, \boldsymbol{\sigma}_{e}^{2}\right) d \mathbf{x}_{t},
\end{aligned}
$$

em que $f\left(\mathbf{x}_{t} \mid \mathbf{w}_{t} ; \boldsymbol{\xi}, \boldsymbol{\sigma}_{e}^{2}\right)$ é a função de densidade condicional de $\mathbf{x}_{t}$ dado $\mathbf{w}_{t}$, ou seja, a função densidade correspondente à distribuição $N_{r}\left(\boldsymbol{\mu}_{x_{t} \mid w_{t}} ; \boldsymbol{\sigma}_{x_{t} \mid w_{t}}^{2}\right)$, em que os elementos de $\boldsymbol{\mu}_{x_{t} \mid w_{t}}$ são $\mu_{x i}+\left[\sigma_{x i}^{2} /\left(\sigma_{x i}^{2}+\sigma_{e i}^{2}\right)\right]\left(w_{t i}-\mu_{x i}\right)$ e $\boldsymbol{\sigma}_{x_{t} \mid w_{t}}^{2}$ é uma matriz diagonal cujo $i$-ésimo elemento da diagonal é $\sigma_{x i}^{2} \sigma_{e i}^{2} /\left(\sigma_{x i}^{2}+\sigma_{e i}^{2}\right)$, para todo $i=1,2, \ldots, r$.

É conveniente utilizar a formulação em (2.11) para o logaritmo da função de verossimilhança, pois os desenvolvimentos computacionais tornam-se mais flexíveis. Notemos que o cálculo do logaritmo da função de verossimilhança é analiticamente inviável dada a presença de integrais múltiplas. Então, algumas metodologias de estimação numérica dos parâmetros são apresentadas a seguir. Con- 
sideramos uma única covariável medida com erro para exemplificar as metodologias abordadas nas subseções seguintes, no entanto, essas metodologias, podem ser generalizadas para $r$ covariáveis de maneira similar.

\subsubsection{Estimação por máxima verossimilhança aproximada}

A função de verossimilhança do modelo de regressão beta com erros de medida é analiticamente intratável devido à presença de integrais que não têm solução explícita. Na literatura existem métodos de integração numérica, por exemplo, os métodos de aproximação via Monte Carlo, Laplace e quadratura de Gauss (Legendre, de Chebyshev de $1^{\underline{a}}$ e $2^{\underline{a}}$ espécie, Leguerre generalizado e Hermite). A quadratura de Gauss-Hermite (QGH) tem sido frequentemente utilizada, como mostram Liu e Pierce (1994), Golub e Welsch (1967), Rabe-Hesketh et al. (2005) e Villegas et al. (2011), entre outros. Esta consiste em realizar a aproximação

$$
\int_{-\infty}^{\infty} e^{-x^{2}} f(x) d x \approx \sum_{q=1}^{Q} \nu_{q} f\left(s_{q}\right)
$$

em que $s_{q}$ e $\nu_{q}$ representam o $q$-ésimo zero e peso, respectivamente, do polinômio ortogonal de Hermite $^{1}$ de ordem $Q$ (número de pontos de quadratura). Mais detalhes sobre o método da quadratura gaussiana hermítica pode ser visto no Apêndice (A.1).

Sejam $\left(y_{1}, \mathbf{w}_{1}\right),\left(y_{2}, \mathbf{w}_{2}\right), \ldots,\left(y_{n}, \mathbf{w}_{n}\right)$ os $n$ pares de variáveis aleatórias observadas. O logaritmo da função de verossimilhança em (2.11), admitindo que uma única covariável é medida com erro, é dado por

$$
\ell(\boldsymbol{\Psi})=\sum_{t=1}^{n} \ell_{1 t}\left(\boldsymbol{\xi}, \sigma_{e}^{2}\right)+\sum_{t=1}^{n} \ell_{2 t}\left(\boldsymbol{\theta}, \boldsymbol{\xi}, \sigma_{e}^{2}\right)
$$

em que

$$
\begin{aligned}
\ell_{1 t}\left(\boldsymbol{\xi}, \sigma_{e}^{2}\right) & =\log f\left(w_{t} ; \boldsymbol{\xi}, \sigma_{e}^{2}\right)=-\frac{1}{2} \log \left[2 \pi\left(\sigma_{x}^{2}+\sigma_{e}^{2}\right)\right]-\frac{1}{2\left(\sigma_{x}^{2}+\sigma_{e}^{2}\right)}\left(w_{t}-\mu_{t}\right)^{2}, \\
\ell_{2 t}\left(\boldsymbol{\theta}, \boldsymbol{\xi}, \sigma_{e}^{2}\right) & =\log \int_{-\infty}^{\infty} f\left(y_{t} \mid x_{t} ; \boldsymbol{\theta}, \boldsymbol{\xi}, \sigma_{e}^{2}\right) f\left(x_{t} \mid w_{t} ; \boldsymbol{\xi}, \sigma_{e}^{2}\right) d x_{t} .
\end{aligned}
$$

O segundo componente do logaritmo da função de verossimilhança em (2.13), que depende da integral, pode ser aproximado pelo método da quadratura de Gauss-Hermite dado em (2.12). Como $f\left(x_{t} \mid w_{t} ; \boldsymbol{\xi}, \sigma_{e}^{2}\right)$ é a função densidade condicional de $x_{t}$ dado $w_{t}$, correspondente à distribuição normal com média $\mu_{x_{t} \mid w_{t}}=\mu_{x}+k_{x}\left(w_{t}-\mu_{x}\right)$ e variância $\sigma_{x_{t} \mid w_{t}}^{2}=\left(1-k_{x}\right) \sigma_{x}^{2}$, sendo $k_{x}=\sigma_{x}^{2} /\left(\sigma_{x}^{2}+\sigma_{e}^{2}\right)$, $\ell_{2 t}\left(\boldsymbol{\theta}, \boldsymbol{\xi}, \sigma_{e}^{2}\right)$ pode ser escrita como

$$
\ell_{2 t}\left(\boldsymbol{\theta}, \boldsymbol{\xi}, \sigma_{e}^{2}\right)=\log \int_{-\infty}^{\infty} f\left(y_{t} \mid x_{t} ; \boldsymbol{\theta}, \boldsymbol{\xi}, \sigma_{e}^{2}\right) \frac{1}{\sqrt{2 \pi \sigma_{x_{t} \mid w_{t}}^{2}}} \exp \left\{-\left(\frac{x_{t}-\mu_{x_{t} \mid w_{t}}}{\sqrt{2 \sigma_{x_{t} \mid w_{t}}^{2}}}\right)^{2}\right\} d x_{t} .
$$

Considerando a seguinte transformação de variáveis, $\check{x}_{t}=\left(x_{t}-\mu_{x_{t} \mid w_{t}}\right) / \sqrt{2 \sigma_{x_{t} \mid w_{t}}^{2}}$, temos $x_{t}=$

\footnotetext{
${ }^{1}$ Abramowitz e Stegun (1972, Seção 20.4.46)
} 
$\sqrt{2 \sigma_{x_{t} \mid w_{t}}^{2}} \check{x}_{t}+\mu_{x_{t} \mid w_{t}}$ e $d x_{t}=\sqrt{2 \sigma_{x_{t} \mid w_{t}}^{2}} d \check{x}_{t}$. Assim, a expressão em (2.14) pode ser escrita como

$$
\ell_{2 t}\left(\boldsymbol{\theta}, \boldsymbol{\xi}, \sigma_{e}^{2}\right)=\log \sum_{q=1}^{Q} \frac{\nu_{q}}{\sqrt{\pi}} \exp \left\{\ell_{t q}\left(\mu_{t q} ; \phi_{t q}\right)\right\}
$$

em que

$$
\begin{aligned}
\ell_{t q}\left(\mu_{t q} ; \phi_{t q}\right)= & \log \Gamma\left(\phi_{t q}\right)-\log \Gamma\left(\mu_{t q} \phi_{t q}\right)-\log \Gamma\left[\left(1-\mu_{t q}\right) \phi_{t q}\right]+\left(\mu_{t q} \phi_{t q}-1\right) \log \left(y_{t}\right)+ \\
& {\left[\left(1-\mu_{t q}\right) \phi_{t q}-1\right] \log \left(1-y_{t}\right) } \\
g\left(\mu_{t q}\right)= & \mathbf{z}_{t}^{\top} \boldsymbol{\alpha}+x_{t}^{*} \beta \\
h\left(\phi_{t q}\right)= & \mathbf{v}_{t}^{\top} \boldsymbol{\gamma}+x_{t}^{*} \lambda \\
x_{t}^{*}= & \mu_{x_{t} \mid w_{t}}+\sqrt{2 \sigma_{x_{t} \mid w_{t}}^{2}} s_{q} \\
\mu_{x_{t} \mid w_{t}}= & \mu_{x}+k_{x}\left(w_{t}-\mu_{x}\right) \\
\sigma_{x_{t} \mid w_{t}}^{2}= & \sigma_{e}^{2} k_{x} \\
k_{x}= & \frac{\sigma_{x}^{2}}{\sigma_{x}^{2}+\sigma_{e}^{2}}
\end{aligned}
$$

Para evitar problemas de identificabilidade nos modelos com erros de medida é usual assumir que a variância do erro de medida $\sigma_{e}^{2}$ é conhecida ou é estimada quando há réplicas para $w_{t}$. Mostrar que o modelo de regressão beta com erros de medida apresenta problemas de identificabilidade é inviável. Nesse sentido, assumiremos conhecida ou estimada quando há réplicas para $w_{t}$ a variância do erro. No que resta desta seção, omitiremos na notação o parâmetro $\sigma_{e}^{2}$. Derivando parcialmente o logaritmo da função de verossimilhança aproximada com respeito ao vetor de parâmetros $\Psi=$ $\left(\boldsymbol{\alpha}^{\top}, \beta, \boldsymbol{\gamma}^{\top}, \lambda, \mu_{x}, \sigma_{x}^{2}\right)^{\top}$, obtemos os componentes do vetor escore aproximado $U_{a}(\boldsymbol{\Psi})=\left(U_{\boldsymbol{\theta}}^{\top}, U_{\boldsymbol{\xi}}^{\top}\right)^{\top}=$ $\left(U_{\boldsymbol{\alpha}}^{\top}, U_{\beta}, U_{\boldsymbol{\gamma}}^{\top}, U_{\lambda}, U_{\mu_{x}}, U_{\sigma_{x}^{2}}\right)^{\top}$. Os elementos do vetor $U_{a}(\Psi)$ podem ser vistos no Apêndice A.2.1. O estimador de máxima verossimilhança aproximada de $\boldsymbol{\Psi}, \widehat{\Psi}$ digamos, é a solução do sistema de equações $U_{\boldsymbol{\alpha}}=\mathbf{0}, U_{\beta}=0, U_{\boldsymbol{\gamma}}=\mathbf{0}, U_{\lambda}=0, U_{\mu_{x}}=0$ e $U_{\sigma_{x}^{2}}=0$, em que a utilização de métodos iterativos de maximização são requeridos.

Para este método de estimação, utilizamos a linguagem de programação Ox para estimar os parâmetros. O programa a ser utilizado para estimar os parâmetros é dado no Apêndice B.1.

Abordaremos no Capítulo 3 algumas propriedades assintóticas dos estimadores de máxima verossimilhança aproximada com o objetivo de construir intervalos de confiança e testar hipóteses.

\subsubsection{Estimação por máxima pseudo-verossimilhança aproximada}

O método de máxima verossimilhança é um dos mais conhecidos e utilizados na estimação dos parâmetros de um modelo estatístico. Cramér (1999, Cap. 28) mostra que as equações de verossimilhança têm raízes fortemente consistentes, assintoticamente eficientes e distribuídas normalmente. No entanto, a aplicação deste método tem-se mostrado inviável em muitas situações particulares, especialmente na presença de parâmetros de perturbação. Vários procedimentos de estimação alternativos têm sido propostos. Dentre estes, há o método de máxima verossimilhança parcial (Cox, 1975), máxima verossimilhança condicional (Godambe, 1976) e máxima pseudo-verossimilhança (Gong e Samaniego, 1981). Em geral, as abordagens de estimação na presença de parâmetros de perturbação estão focadas em eliminá-los. Vários exemplos são apresentados por Pace e Salvan 
(1997, Cap. 4).

Nesta seção utilizamos o método de máxima pseudo-verossimilhança com o intuito de contornar a presença dos parâmetros de perturbação referentes às distribuições da variável medida com erros ou sua substituta. A ideia central deste método é substituir o vetor de parâmetros de perturbação por uma estimativa consistente na função de máxima verossimilhança original (2.13), gerando assim uma pseudo-verossimilhança que depende somente dos parâmetros de interesse. As estimativas de máxima pseudo-verossimilhança poderão ser calculadas por algum método confiável. Nosso objetivo é explorar esse método no modelo de regressão beta com erros nas variáveis tratado neste capítulo.

Sejam $\left(\boldsymbol{\xi}^{\top}, \sigma_{e}^{2}\right)^{\top}=\left(\mu_{x}, \sigma_{x}^{2}, \sigma_{e}^{2}\right)^{\top}$ o vetor de parâmetros de perturbação e $\boldsymbol{\theta}=\left(\boldsymbol{\alpha}^{\top}, \beta, \boldsymbol{\gamma}^{\top}, \lambda\right)^{\top}$ o vetor de parâmetros de interesse em (2.9). Como não parece possível eliminar o vetor $\boldsymbol{\xi}$ em (2.13) através de integração, utilizaremos a abordagem de pseudo-verossimilhança (Gong e Samaniego, 1981; Guolo, 2011). Assim, o logaritmo da função verossimilhança (2.13) é maximizado em duas etapas. Na primeira, como sugerem Buonaccorsi e Tosteson (1993) e Guolo (2011), estimamos o vetor de parâmetros de perturbação $\boldsymbol{\xi}$ a partir da maximização do logaritmo da função de verossimilhança reduzida

$$
\ell_{r}(\boldsymbol{\xi} ; w)=\sum_{t=1}^{n} \log f\left(w ; \boldsymbol{\xi}, \sigma_{e}^{2}\right)
$$

em que a variância do erro de medição, $\sigma_{e}^{2}$, é assumida conhecida ou é estimada quando existem réplicas para $w_{t}$. Na segunda etapa a estimativa $\widehat{\boldsymbol{\xi}}$ obtida da maximização de (2.15) é substituída no logaritmo da função de verossimilhança original (2.13), o que resulta na log-pseudo-verossimilhança

$$
\ell_{p}\left(\boldsymbol{\theta} ; \widehat{\boldsymbol{\xi}}, \widehat{\sigma}_{e}^{2}\right)=\sum_{t=1}^{n} \ell_{1 t}\left(\widehat{\boldsymbol{\xi}}, \widehat{\sigma}_{e}^{2}\right)+\sum_{t=1}^{n} \ell_{2 t}\left(\boldsymbol{\theta}, \widehat{\boldsymbol{\xi}}, \widehat{\sigma}_{e}^{2}\right)
$$

sendo que $\widehat{\sigma}_{e}^{2}$ representa o valor conhecido ou estimado de $\sigma_{e}^{2}$.

Podemos observar que, assim como $\ell_{a}(\Psi), \ell_{p}\left(\boldsymbol{\theta} ; \widehat{\boldsymbol{\xi}}, \widehat{\sigma}_{e}^{2}\right)$ é analiticamente intratável. No entanto, ao contrário do que ocorre $\operatorname{com} \ell_{a}(\boldsymbol{\Psi})$, em $\ell_{p}\left(\boldsymbol{\theta} ; \widehat{\boldsymbol{\xi}}, \widehat{\sigma}_{e}^{2}\right)$ a parcela que envolve uma integral depende apenas dos parâmetros de interesse. A maximização de $\ell_{p}\left(\boldsymbol{\theta} ; \widehat{\boldsymbol{\xi}}, \widehat{\sigma}_{e}^{2}\right)$ também será solucionado pelo método de quadratura de Gauss-Hermite como em (2.2.1). Assim, o logaritmo da pseudoverossimilhança aproximada para o modelo de regressão beta com erros de medida unidimensional é dado por (2.16), em que

$$
\begin{aligned}
\ell_{1 t}\left(\widehat{\boldsymbol{\xi}}, \widehat{\sigma}_{e}^{2}\right) & =-\frac{n}{2} \log \left[2 \pi\left(\widehat{\sigma}_{x}^{2}+\widehat{\sigma}_{e}^{2}\right)\right]-\frac{1}{2\left(\widehat{\sigma}_{x}^{2}+\widehat{\sigma}_{e}^{2}\right)} \sum_{t=1}^{n}\left(w_{t}-\widehat{\mu}_{x}\right)^{2}, \\
\ell_{2 t}\left(\boldsymbol{\theta}, \widehat{\boldsymbol{\xi}}, \widehat{\sigma}_{e}^{2}\right) & =\sum_{t=1}^{n} \log \left(\sum_{q=1}^{Q} \frac{\nu_{q}}{\sqrt{\pi}} \exp \left\{\ell_{t q}\left(\boldsymbol{\theta} ; \widehat{\boldsymbol{\xi}}, \widehat{\sigma}_{e}^{2}\right)\right\}\right),
\end{aligned}
$$


com

$$
\begin{aligned}
\ell_{t q}\left(\boldsymbol{\theta} ; \widehat{\boldsymbol{\xi}}, \widehat{\sigma}_{e}^{2}\right)= & \log \Gamma\left(\phi_{t q}\right)-\log \Gamma\left(\mu_{t q} \phi_{t q}\right)-\log \Gamma\left[\left(1-\mu_{t q}\right) \phi_{t q}\right]+\left(\mu_{t q} \phi_{t q}-1\right) \log \left(y_{t}\right)+ \\
& {\left[\left(1-\mu_{t q}\right) \phi_{t q}-1\right] \log \left(1-y_{t}\right) } \\
g\left(\mu_{t q}\right)= & \mathbf{z}_{t}^{\top} \boldsymbol{\alpha}+\widehat{x}_{t}^{*} \beta \\
h\left(\phi_{t q}\right)= & \mathbf{v}_{t}^{\top} \gamma+\widehat{x}_{t}^{*} \lambda \\
\widehat{x}_{t}^{*}= & \widehat{\mu}_{x_{t} \mid w_{t}}+\sqrt{2 \widehat{\sigma}_{x_{t} \mid w_{t}}^{2}} s_{q} \\
\widehat{\mu}_{x_{t} \mid w_{t}}= & \mu_{x}+\widehat{k}_{x}\left(w_{t}-\widehat{\mu}_{x}\right) \\
\widehat{\sigma}_{x_{t} \mid w_{t}}^{2}= & \left(1-\widehat{k}_{x}\right) \widehat{\sigma}_{x}^{2} \\
\widehat{k}_{x}= & \frac{\widehat{\sigma}_{x}^{2}}{\widehat{\sigma}_{x}^{2}+\widehat{\sigma}_{e}^{2}}
\end{aligned}
$$

em que $s_{q}$ e $\nu_{q}$ representam o $q$-ésimo zero e peso, respectivamente, do polinômio ortogonal de Hermite de ordem $Q$.

Derivando parcialmente $\ell_{p}\left(\boldsymbol{\theta} ; \widehat{\boldsymbol{\xi}}, \widehat{\sigma}_{e}^{2}\right)$ com respeito ao vetor de parâmetros de interesse $\boldsymbol{\theta}=$ $\left(\boldsymbol{\alpha}^{\top}, \beta, \boldsymbol{\gamma}^{\top}, \lambda\right)^{\top}$, obtemos os componentes do vetor pseudo-escore aproximado representado por $U_{p}(\boldsymbol{\theta})=\left(U_{\boldsymbol{\alpha}}^{\top}, U_{\beta}, U_{\boldsymbol{\gamma}}^{\top}, U_{\lambda}\right)^{\top}$, cujos elementos são similares aos obtidos no Apêndice A.2.1, sendo que $\widehat{\mu}_{x}$ e $\widehat{\sigma}_{x}^{2}$ são estimativas obtidas a partir do logaritmo da função de verossimilhança restrita $\ell_{r}(\boldsymbol{\xi} ; w)$. O estimador de máxima pseudo-verossimilhança de $\boldsymbol{\theta}, \widehat{\boldsymbol{\theta}}$, é solução simultânea do sistema de equações $U_{\boldsymbol{\alpha}}=\mathbf{0}, U_{\beta}=0, U_{\boldsymbol{\gamma}}=\mathbf{0}$ e $U_{\lambda}=0$, que requer também a utilização de métodos iterativos de maximização. Utilizando a linguagem de programação ox, apresentamos no Apêndice B.2 os passos para estimar os parâmetros.

No capítulo seguinte apresentaremos algumas propriedades assintóticas dos estimadores seguindo Gong e Samaniego (1981), Carroll et al. (2006, Seção A.6.6) e Guolo (2011).

\subsubsection{Estimação por calibração da regressão}

Nos últimos anos o método de calibração da regressão vem sendo muito utilizado por sua simplicidade na estimação de parâmetros quando se assume erros de medição nas variáveis preditoras, como em Carroll et al. (2006, Cap.4), Freedman et al. (2008), Thurston et al. (2005) e Guolo (2011). A ideia central deste método é substituir a variável não observável, $x_{t}$, por uma estimativa da esperança condicional de $x_{t}$ dado $w_{t}, \mathrm{E}\left(x_{t} \mid w_{t}\right)$. Após a substituição, as estimativas dos parâmetros são obtidas por algum método confiável, por exemplo, por máxima verossimilhança (Carroll et al., 2006, p.65). Seja $r\left(w_{t}, \boldsymbol{\psi}\right)=\mathrm{E}\left(x_{t} \mid w_{t}\right)$, a função de calibração, que depende de um vetor de parâmetros $\boldsymbol{\psi}$. A troca da variável não observada $x_{t}$ por $r\left(w_{t}, \widehat{\boldsymbol{\psi}}\right)$ estabelece um modelo modificado para os dados. Aqui, $\widehat{\boldsymbol{\psi}}$ é uma estimativa de $\boldsymbol{\psi}$.

Para o caso do modelo de regressão beta com erros de medida sob o enfoque estrutural dado pelas equações $(2.1),(2.4),(2.5)$ e (2.7) com uma única variável medida com erro, temos que a função de calibração é

$$
r\left(w_{t}, \boldsymbol{\psi}\right)=\mu_{x}+k_{x}\left(w_{t}-\mu_{x}\right)
$$

em que $\boldsymbol{\psi}=\left(\mu_{x}, \sigma_{x}^{2}, \sigma_{e}^{2}\right)^{\top}$ e o quociente $k_{x}=\sigma_{x}^{2} /\left(\sigma_{x}^{2}+\sigma_{e}^{2}\right)$ é conhecido como razão de confiabilidade. Como $w_{t} \sim N\left(\mu_{x}, \sigma_{x}^{2}+\sigma_{e}^{2}\right)$, então, $\bar{w}=\sum_{t=1}^{n} w_{t} / n$ e $s_{w}^{2}=\sum_{t=1}^{n}\left(w_{t}-\bar{w}\right)^{2} /(n-1)$ são estimadores 
ótimos de $\mu_{x}$ e $\sigma_{x}^{2}+\sigma_{e}^{2}$, respectivamente. Entretanto, não é possível estimar $\sigma_{x}^{2}$ através dos dados observados $w_{1}, \ldots, w_{n}$. Assumimos, então, que $k_{x}$ ou $\sigma_{e}^{2}$ é conhecido ou, alternativamente, que $\sigma_{e}^{2}$ pode ser estimado desde que seja possível observar réplicas de $w_{t}$.

Substituindo a função de calibração estimada na função densidade de probabilidade de $y_{t}$ dado $x_{t}$, ou seja, em $f\left(y_{t} \mid x_{t} ; \boldsymbol{\alpha}, \beta, \gamma, \lambda\right)$ dado em (2.1), obtemos o logaritmo da função de verossimilhança modificada

$$
\ell_{R C}(\boldsymbol{\theta})=\sum_{t=1}^{n} \ell_{t}\left(\mu_{t}, \phi_{t}\right),
$$

em que

$$
\begin{aligned}
\ell_{t}\left(\mu_{t}, \phi_{t}\right)= & \log \Gamma\left(\phi_{t}\right)-\log \Gamma\left(\mu_{t} \phi_{t}\right)-\log \Gamma\left[\left(1-\mu_{t}\right) \phi_{t}\right]+\left(\mu_{t} \phi_{t}-1\right) \log \left(y_{t}\right)+ \\
& {\left[\left(1-\mu_{t}\right) \phi_{t}-1\right] \log \left(1-y_{t}\right), } \\
g\left(\mu_{t}\right)= & \mathbf{z}_{t}^{\top} \boldsymbol{\alpha}+x_{t}^{*} \beta, \\
h\left(\phi_{t}\right)= & \mathbf{v}_{t}^{\top} \boldsymbol{\gamma}+x_{t}^{*} \lambda, \\
x_{t}^{*}= & \bar{w}+\widehat{k}_{x}\left(w_{t}-\bar{w}\right),
\end{aligned}
$$

em que $\widehat{k}_{x}$ é o valor conhecido ou estimado de $k_{x}$.

Nota-se que o logaritmo da função de verossimilhança modificada depende apenas do parâmetro de interesse $\boldsymbol{\theta}$ e que coincide com o do modelo de regressão beta usual, definido na Seção 2, uma vez que $x_{t}^{*}$ atua como uma variável explicativa, medida sem erro. Esse fato é relevante, pois a estimação dos parâmetros por máxima verossimilhança pode ser realizada sem dificuldade utilizando, por exemplo, o pacote betareg (Cribari-Neto e Zeiles, 2010) disponível na plataforma $\mathrm{R}$.

Derivando parcialmente $\ell_{R C}(\boldsymbol{\theta})$ com respeito ao vetor de parâmetros de interesse $\boldsymbol{\theta}$, obtemos os componentes do vetor escore $U_{R C}(\boldsymbol{\theta})=\left(U_{\boldsymbol{\alpha}}^{\top}, U_{\beta}, U_{\boldsymbol{\gamma}}^{\top}, U_{\lambda}\right)^{\top}$. O estimador de máxima verossimilhança de $\boldsymbol{\theta}, \widehat{\boldsymbol{\theta}}$, é solução simultânea do sistema de equações $U_{\boldsymbol{\alpha}}=\mathbf{0}, U_{\beta}=0, U_{\boldsymbol{\gamma}}=\mathbf{0}$ e $U_{\lambda}=0$, que requerem a utilização de métodos iterativos de maximização.

Como foi dito anteriormente, é possível utilizar o pacote betareg para estimar os parâmetros usando o sistema R (Cribari-Neto e Zeiles, 2010). Uma outra alternativa é a utilização da linguagem de programação Ox. Apresentamos no Apêndice B.3 o respectivo programa que estima o parâmetros. Neste programa utilizamos a função log_Naive.ox dada no Apêndice B.13.

No capítulo seguinte apresentaremos algumas propriedades assintóticas dos estimadores segundo Gong e Samaniego (1981). Estas propriedades são necessárias para a construção de intervalos de confiança e para testar hipóteses.

Com o objetivo de investigar o comportamento dos estimadores dos parâmetros do modelo de regressão beta na presença de erros de medida realizamos a seguir um estudo de simulação em cenários em que diferentes valores para a variância do erro e de tamanhos de amostra foram considerados.

\subsection{Estudo de simulação}

Realizamos um estudo de simulação de Monte Carlo com $R=10000$ réplicas objetivando investigar o comportamento dos estimadores dos parâmetros do modelo obtidos pelos métodos descritos 
nas seções anteriores. Para $\Psi=\left(\Psi_{1}, \Psi_{2}, \Psi_{3}, \Psi_{4}, \Psi_{5}, \Psi_{6}\right)^{\top}=\left(\alpha, \beta, \gamma, \lambda, \mu_{x}, \sigma_{x}^{2}\right)^{\top}$, temos que

$$
\bar{\Psi}_{j}=\frac{1}{R} \sum_{i=1}^{R} \widehat{\Psi}_{j}^{(i)}, \quad \operatorname{Viés}\left(\Psi_{j}\right)=\bar{\Psi}_{j}-\Psi_{j} \quad \text { e } \operatorname{REQM}\left(\Psi_{j}\right)=\sqrt{\frac{1}{R} \sum_{i=1}^{R}\left(\widehat{\Psi}_{j}^{(i)}-\Psi_{j}\right)^{2}},
$$

para $j=1, \ldots, 6$, são, respectivamente, estimativas da média, do viés e da raiz do erro quadrático médio dos estimadores dos parâmetros. Aqui $\widehat{\Psi}_{j}^{(i)}$ representa a estimativa de $\Psi_{j}$ obtida na $i$-ésima amostra simulada. Amostras de tamanhos $n=25, n=50$ e $n=100$ foram consideradas. Geramos, para cada réplica de Monte Carlo, a variável não observada $x_{t}$ com distribuição normal com média $\mu_{x}$ e variância $\sigma_{x}^{2}$. As ligações logito e logarítmica foram escolhidas, respectivamente, para os parâmetros $\mu_{t}$ e $\phi_{t}$ obtidos a partir das equações (2.4) e (2.5). Geramos a variável dependente $y_{t}$ a partir de uma distribuição beta com média $\mu_{t}$ e precisão $\phi_{t}$. Além disso, geramos a covariável $w_{t}$ a partir de sua distribuição condicional dado $x_{t}$, ou seja, com uma distribuição normal com média $x_{t}$ e variância $\sigma_{e}^{2}$. Consideramos que a variância do erro do modelo $\sigma_{e}^{2}$ é conhecida e atribuímos os seguintes valores a $\sigma_{e}^{2}: 0,05,0,50$ e 1,0. Para cada amostra simulada, os parâmetros do modelo foram estimados sob dois enfoques: primeiro, assumimos (erroneamente) que não há erros de medida na covariável $x_{t}$, ou seja, utilizamos o método naïve; segundo, reconhecemos que $x_{t}$ é medida com erros e, nesse caso, utilizamos os três métodos descritos nas seções anteriores, a saber: máxima verossimilhança aproximada, máxima pseudo-verossimilhança aproximada e calibração da regressão. Analisaremos quatro diferentes cenários descritos a seguir para o modelo de regressão beta com erros de medida sob o enfoque estrutural. Nos três primeiros cenários consideramos $Q=12$ como o número de pontos de quadratura para aproximar os métodos de estimação vistos na Seção 2.2. No entanto, no cenário 4 estudamos a influência do número de pontos de quadratura na estimação dos parâmetros; para tal efeito atribuímos os seguintes valores a $Q: 12,24$ e 48 .

\subsubsection{Cenário 1}

Neste cenário admitimos que o modelo de regressão beta com erros de medida sob o enfoque estrutural apresenta a estrutura

$$
\begin{aligned}
g\left(\mu_{t}\right) & =\alpha+\beta x_{t}, \\
h\left(\phi_{t}\right) & =\gamma+\lambda x_{t}, \\
w_{t} & =x_{t}+e_{t},
\end{aligned}
$$

em que $t=1,2, \ldots, n$. Escolhemos os seguintes valores para os parâmetros que permanecem fixos para todas as simulações: $\alpha=2,0, \beta=-0,6, \gamma=2,5, \lambda=0,5, \mu_{x}=2,5$ e $\sigma_{x}^{2}=2,7$. Estes valores conduzem a que a média da variável resposta pertença ao intervalo $(0,103 ; 0,954)$ e o grau de heterocedasticidade, $\varrho=\max \left(\phi_{t}\right) / \min \left(\phi_{t}\right)$, seja aproximadamente 75,704 .

As Tabelas 2.1, 2.2 e 2.3 mostram os resultados da simulação obtidos neste cenário para diferentes valores de $\sigma_{e}^{2}$, a variância do erro de medida. Notamos que os estimadores obtidos pelos métodos aproximados de máxima verossimilhança $\left(\ell_{a}\right)$ e pseudo-verossimilhança $\left(\ell_{p}\right)$ têm comportamentos semelhantes e são claramente melhores que os outros dois. Conforme esperado, à medida em que $n$ cresce, o viés e o erro quadrático médio de tais estimadores diminuem. Os estimadores naive ( $\left.\ell_{\text {naive }}\right)$ mantêm viés considerável mesmo em amostras grandes e especialmente quando a variância do erro de medida é grande. Esta mesma tendência, porém menos pronunciada, é observada para 
Tabela 2.1: Média, Viés e REQM das estimativas de Monte Carlo dos parâmetros com variância do erro de medida $\sigma_{e}^{2}=0,05$. Verdadeiros valores: $\alpha=2,0, \beta=-0,6, \gamma=2,5, \lambda=0,5, \mu_{x}=2,5$ e $\sigma_{x}^{2}=2,7$. Cenário 1 .

\begin{tabular}{|c|c|c|c|c|c|c|}
\hline$n$ & Método & & $\alpha$ & $\beta$ & $\gamma$ & $\lambda$ \\
\hline \multirow{12}{*}{25} & \multirow{3}{*}{$\ell_{a}$} & Média & 2,00 & $-0,60$ & 2,50 & 0,58 \\
\hline & & Viés & 0,00 & 0,00 & 0,00 & 0,08 \\
\hline & & REQM & 0,19 & 0,05 & 0,28 & 0,23 \\
\hline & \multirow{3}{*}{$\ell_{p}$} & Média & 2,00 & $-0,60$ & 2,51 & 0,58 \\
\hline & & Viés & 0,00 & 0,00 & 0,01 & 0,08 \\
\hline & & REQM & 0,19 & 0,05 & 0,27 & 0,22 \\
\hline & \multirow{3}{*}{$\ell_{R C}$} & Média & 1,99 & $-0,60$ & 2,47 & 0,47 \\
\hline & & Viés & $-0,01$ & 0,00 & $-0,03$ & $-0,03$ \\
\hline & & REQM & 0,45 & 0,17 & 0,45 & 0,22 \\
\hline & \multirow{3}{*}{$\ell_{\text {Nainve }}$} & Média & 1,97 & $-0,59$ & 2,49 & 0,45 \\
\hline & & Viés & $-0,03$ & 0,01 & $-0,01$ & $-0,05$ \\
\hline & & REQM & 0,19 & 0,05 & 0,29 & 0,21 \\
\hline \multirow{12}{*}{50} & \multirow{3}{*}{$\ell_{a}$} & Média & 2,00 & $-0,60$ & 2,50 & 0,54 \\
\hline & & Viés & 0,00 & 0,00 & 0,00 & 0,04 \\
\hline & & REQM & 0,13 & 0,03 & 0,25 & 0,13 \\
\hline & \multirow{3}{*}{$\ell_{p}$} & Média & 2,00 & $-0,60$ & 2,50 & 0,54 \\
\hline & & Viés & 0,00 & 0,00 & 0,00 & 0,04 \\
\hline & & REQM & 0,13 & 0,04 & 0,26 & 0,13 \\
\hline & \multirow{3}{*}{$\ell_{R C}$} & Média & 1,99 & $-0,59$ & 2,48 & 0,45 \\
\hline & & Viés & $-0,01$ & 0,01 & $-0,02$ & $-0,05$ \\
\hline & & REQM & 0,32 & 0,12 & 0,33 & 0,17 \\
\hline & \multirow{3}{*}{$\ell_{\text {Naïve }}$} & Média & 1,97 & $-0,59$ & 2,49 & 0,43 \\
\hline & & Viés & $-0,03$ & 0,01 & $-0,01$ & $-0,07$ \\
\hline & & REQM & 0,13 & 0,03 & 0,25 & 0,18 \\
\hline \multirow{12}{*}{100} & \multirow{3}{*}{$\ell_{a}$} & Média & 2,00 & $-0,60$ & 2,51 & 0,52 \\
\hline & & Viés & 0,00 & 0,00 & 0,01 & 0,02 \\
\hline & & REQM & 0,09 & 0,02 & 0,22 & 0,09 \\
\hline & \multirow{3}{*}{$\ell_{p}$} & Média & 2,00 & $-0,60$ & 2,51 & 0,52 \\
\hline & & Viés & 0,00 & 0,00 & 0,01 & 0,02 \\
\hline & & REQM & 0,09 & 0,02 & 0,23 & 0,09 \\
\hline & \multirow{3}{*}{$\ell_{R C}$} & Média & 1,99 & $-0,59$ & 2,48 & 0,44 \\
\hline & & Viés & $-0,01$ & 0,01 & $-0,02$ & $-0,06$ \\
\hline & & REQM & 0,23 & 0,09 & 0,26 & 0,13 \\
\hline & \multirow{3}{*}{$\ell_{\text {Nä̈ve }}$} & Média & 1,97 & $-0,59$ & 2,50 & 0,42 \\
\hline & & Viés & $-0,03$ & 0,01 & 0,00 & $-0,08$ \\
\hline & & REQM & 0,09 & 0,03 & 0,21 & 0,15 \\
\hline
\end{tabular}


Tabela 2.2: Média, Viés e REQM das estimativas de Monte Carlo dos parâmetros com variância do erro de medida $\sigma_{e}^{2}=0,50$. Verdadeiros valores: $\alpha=2,0, \beta=-0,6, \gamma=2,5, \lambda=0,5, \mu_{x}=2,5$ e $\sigma_{x}^{2}=2,7$. Cenário 1

\begin{tabular}{|c|c|c|c|c|c|c|}
\hline$n$ & Método & & $\alpha$ & $\beta$ & $\gamma$ & $\lambda$ \\
\hline \multirow{12}{*}{25} & \multirow{3}{*}{$\ell_{a}$} & Média & 1,99 & $-0,60$ & 2,47 & 0,70 \\
\hline & & Viés & 0,01 & 0,00 & $-0,03$ & 0,20 \\
\hline & & REQM & 0,29 & 0,08 & 0,40 & 0,41 \\
\hline & \multirow{3}{*}{$\ell_{p}$} & Média & 1,97 & $-0,59$ & 2,50 & 0,68 \\
\hline & & Viés & $-0,03$ & 0,01 & 0,00 & 0,18 \\
\hline & & REQM & 0,31 & 0,09 & 0,27 & 0,37 \\
\hline & \multirow{3}{*}{$\ell_{R C}$} & Média & 1,95 & $-0,58$ & 2,15 & 0,25 \\
\hline & & Viés & $-0,05$ & 0,02 & $-0,35$ & $-0,25$ \\
\hline & & REQM & 0,47 & 0,17 & 0,58 & 0,35 \\
\hline & \multirow{3}{*}{$\ell_{\text {Naïve }}$} & Média & 1,71 & $-0,49$ & 2,28 & 0,22 \\
\hline & & Viés & $-0,29$ & 0,11 & $-0,22$ & $-0,28$ \\
\hline & & REQM & 0,37 & 0,13 & 0,41 & 0,34 \\
\hline \multirow{12}{*}{50} & \multirow{3}{*}{$\ell_{a}$} & Média & 2,00 & $-0,60$ & 2,49 & 0,60 \\
\hline & & Viés & 0,00 & 0,00 & $-0,01$ & 0,10 \\
\hline & & REQM & 0,20 & 0,06 & 0,29 & 0,27 \\
\hline & \multirow{3}{*}{$\ell_{p}$} & Média & 1,98 & $-0,60$ & 2,50 & 0,60 \\
\hline & & Viés & $-0,02$ & 0,00 & 0,00 & 0,10 \\
\hline & & REQM & 0,21 & 0,06 & 0,27 & 0,26 \\
\hline & \multirow{3}{*}{$\ell_{R C}$} & Média & 1,95 & $-0,58$ & 2,16 & 0,23 \\
\hline & & Viés & $-0,05$ & 0,02 & $-0,34$ & $-0,27$ \\
\hline & & REQM & 0,34 & 0,12 & 0,47 & 0,32 \\
\hline & \multirow{3}{*}{$\ell_{\text {Naïve }}$} & Média & 1,72 & $-0,49$ & 2,27 & 0,20 \\
\hline & & Viés & $-0,28$ & 0,11 & $-0,23$ & $-0,30$ \\
\hline & & REQM & 0,32 & 0,12 & 0,35 & 0,32 \\
\hline \multirow{12}{*}{100} & \multirow{3}{*}{$\ell_{a}$} & Média & 2,00 & $-0,60$ & 2,50 & 0,56 \\
\hline & & Viés & 0,00 & 0,00 & 0,00 & 0,06 \\
\hline & & REQM & 0,14 & 0,04 & 0,25 & 0,19 \\
\hline & \multirow{3}{*}{$\ell_{p}$} & Média & 1,99 & $-0,59$ & 2,51 & 0,55 \\
\hline & & Viés & $-0,01$ & 0,00 & 0,01 & 0,05 \\
\hline & & REQM & 0,15 & 0,04 & 0,25 & 0,19 \\
\hline & \multirow{3}{*}{$\ell_{R C}$} & Média & 1,95 & $-0,58$ & 2,16 & 0,23 \\
\hline & & Viés & $-0,05$ & 0,02 & $-0,34$ & $-0,27$ \\
\hline & & REQM & 0,24 & 0,09 & 0,43 & 0,30 \\
\hline & \multirow{3}{*}{$\ell_{\text {Naïve }}$} & Média & 1,72 & $-0,49$ & 2,26 & 0,21 \\
\hline & & Viés & $-0,28$ & 0,11 & $-0,24$ & $-0,29$ \\
\hline & & REQM & 0,30 & 0,11 & 0,32 & 0,31 \\
\hline
\end{tabular}


Tabela 2.3: Média, Viés e REQM das estimativas de Monte Carlo dos parâmetros com variância do erro de medida $\sigma_{e}^{2}=1,0$. Verdadeiros valores: $\alpha=2,0, \beta=-0,6, \gamma=2,5, \lambda=0,5, \mu_{x}=2,5$ e $\sigma_{x}^{2}=2,7$. Cenário 1

\begin{tabular}{|c|c|c|c|c|c|c|}
\hline$n$ & Método & & $\alpha$ & $\beta$ & $\gamma$ & $\lambda$ \\
\hline \multirow{12}{*}{25} & \multirow{3}{*}{$\ell_{a}$} & Média & 2,01 & $-0,60$ & 2,45 & 0,72 \\
\hline & & Viés & 0,01 & 0,00 & $-0,05$ & 0,22 \\
\hline & & REQM & 0,37 & 0,12 & 0,54 & 0,41 \\
\hline & \multirow{3}{*}{$\ell_{p}$} & Média & 1,98 & $-0,60$ & 2,49 & 0,67 \\
\hline & & Viés & $-0,02$ & 0,00 & $-0,01$ & 0,17 \\
\hline & & REQM & 0,37 & 0,11 & 0,28 & 0,33 \\
\hline & \multirow{3}{*}{$\ell_{R C}$} & Média & 1,93 & $-0,58$ & 1,94 & 0,19 \\
\hline & & Viés & $-0,07$ & 0,02 & $-0,56$ & $-0,31$ \\
\hline & & REQM & 0,49 & 0,18 & 0,76 & 0,41 \\
\hline & \multirow{3}{*}{$\ell_{\text {Naïve }}$} & Média & 1,52 & $-0,41$ & 2,12 & 0,16 \\
\hline & & Viés & $-0,48$ & 0,19 & $-0,38$ & $-0,34$ \\
\hline & & REQM & 0,54 & 0,20 & 0,54 & 0,38 \\
\hline \multirow{12}{*}{50} & \multirow{3}{*}{$\ell_{a}$} & Média & 2,00 & $-0,60$ & 2,48 & 0,59 \\
\hline & & Viés & 0,00 & 0,00 & $-0,02$ & 0,09 \\
\hline & & REQM & 0,25 & 0,08 & 0,32 & 0,25 \\
\hline & \multirow{3}{*}{$\ell_{p}$} & Média & 1,98 & $-0,60$ & 2,49 & 0,57 \\
\hline & & Viés & $-0,02$ & 0,00 & $-0,01$ & 0,07 \\
\hline & & REQM & 0,26 & 0,07 & 0,27 & 0,23 \\
\hline & \multirow{3}{*}{$\ell_{R C}$} & Média & 1,92 & $-0,57$ & 1,94 & 0,16 \\
\hline & & Viés & $-0,08$ & 0,03 & $-0,56$ & $-0,34$ \\
\hline & & REQM & 0,35 & 0,13 & 0,66 & 0,40 \\
\hline & \multirow{3}{*}{$\ell_{\text {Naïve }}$} & Média & 1,52 & $-0,42$ & 2,09 & 0,14 \\
\hline & & Viés & $-0,48$ & 0,18 & $-0,41$ & $-0,36$ \\
\hline & & REQM & 0,51 & 0,19 & 0,50 & 0,38 \\
\hline \multirow{12}{*}{100} & \multirow{3}{*}{$\ell_{a}$} & Média & 1,99 & $-0,59$ & 2,50 & 0,53 \\
\hline & & Viés & $-0,01$ & 0,01 & 0,00 & 0,03 \\
\hline & & REQM & 0,17 & 0,06 & 0,27 & 0,17 \\
\hline & \multirow{3}{*}{$\ell_{p}$} & Média & 1,99 & $-0,60$ & 2,50 & 0,52 \\
\hline & & Viés & $-0,01$ & 0,00 & 0,00 & 0,02 \\
\hline & & REQM & 0,18 & 0,05 & 0,26 & 0,17 \\
\hline & \multirow{3}{*}{$\ell_{R C}$} & Média & 1,92 & $-0,57$ & 1,93 & 0,13 \\
\hline & & Viés & $-0,08$ & 0,03 & $-0,57$ & $-0,37$ \\
\hline & & REQM & 0,26 & 0,10 & 0,63 & 0,42 \\
\hline & \multirow{3}{*}{$\ell_{\text {Nä̈ve }}$} & Média & 1,53 & $-0,42$ & 2,07 & 0,14 \\
\hline & & Viés & $-0,47$ & 0,18 & $-0,43$ & $-0,36$ \\
\hline & & REQM & 0,49 & 0,19 & 0,48 & 0,37 \\
\hline
\end{tabular}


os estimadores obtidos por calibração da regressão $\left(\ell_{R C}\right)$.

\subsubsection{Cenário 2}

Neste cenário, assumimos que o modelo de regressão beta com erros de medida sob o enfoque estrutural tem a seguinte especificação:

$$
\begin{aligned}
g\left(\mu_{t}\right) & =\alpha+\beta x_{t}, \\
h\left(\phi_{t}\right) & =\gamma+\lambda x_{t}, \\
w_{t 1 j} & =x_{t}+e_{t},
\end{aligned}
$$

em que $t=1,2, \ldots, n$ e $j=1, \ldots, n_{t 1}$ é o número de réplicas da variável observada com erros. Assumimos que os verdadeiros valores dos parâmetros são $\alpha=2,0, \beta=-0,6, \gamma=2,5, \lambda=0,5$, $\mu_{x}=2,5$ e $\sigma_{x}^{2}=2,7$, iguais aos considerados no cenário 1 . Neste cenário, dada a presença de réplicas de $w_{t}$ podemos obter uma estimativas para $\sigma_{e}^{2}$ tal como é mostrado em (2.1). Consideramos $n_{t 1}=3$, ou seja, $w_{t 1}, w_{t 2}$ e $w_{t 3}$, geradas a partir da uma distribuição normal com média $x_{t}$ e variância $\sigma_{e}^{2}$. Os resultados obtidos neste cenário são apresentados nas Tabelas 2.4, 2.5 e 2.6 para diferentes valores de $\sigma_{e}^{2}\left(\sigma_{e}^{2}=0,05 ; 0,50 ; 1,0\right)$.

Notamos que os resultados obtidos são bem similares aos obtidos sob o cenário 1. Assim, os estimadores dos parâmetros obtidos pelos métodos aproximados de máxima verossimilhança e pseudoverossimilhança tiveram melhor desempenho que os outros dois (naïve e calibração da regressão).

\subsubsection{Cenário 3}

Neste cenário, supomos que o modelo de regressão beta com erros de medida segue a estrutura

$$
\begin{aligned}
g\left(\mu_{t}\right) & =\alpha+\beta_{1} x_{1 t}+\beta_{2} x_{2 t}, \\
h\left(\phi_{t}\right) & =\gamma+\lambda x_{1 t}, \\
w_{1 t} & =x_{1 t}+e_{1 t}, \\
w_{2 t} & =x_{2 t}+e_{2 t},
\end{aligned}
$$

em que $t=1,2, \ldots, n$. Note que assumimos a presença de duas covariáveis independentes medidas com erro, $w_{1 t}$ e $w_{2 t}$. Como nosso foco de trabalho é o enfoque estrutural suponhamos que a distribuição das covariáveis não observadas $x_{1 t}$ e $x_{2 t}$ estão normalmente distribuídas, ou seja, $x_{1 t}, \sim N\left(\mu_{x 1}, \sigma_{x 2 t}^{2}\right)$ e $x_{2 t} \sim N\left(\mu_{x 2}, \sigma_{x 2}^{2}\right)$. Consideramos que os valores verdadeiros dos parâmetros são: $\alpha=2,0, \beta_{1}=-0,6, \beta_{2}=-0,5, \gamma=2,5, \lambda=0,5, \mu_{x 1}=\mu_{x 2}=2,5, \sigma_{x 1}^{2}=\sigma_{x 2}^{2}=2,7$ e $\sigma_{e 1}^{2}=\sigma_{e 2}^{2}=\sigma_{e}^{2}$. Os resultados deste cenário são apresentados nas Tabelas 2.7, 2.8 e 2.9 para diferentes valores de $\sigma_{e}^{2}(0,05 ; 0,50 ; 1,0)$.

Observamos novamente que os estimadores obtidos pelos métodos aproximados de máxima verossimilhança e pseudo-verossimilhança apresentam desempenhos semelhantes e claramente superiores aos dos demais estimadores (naive e calibração da regressão).

\subsubsection{Cenário 4}

Vimos na Seção 2.2 que o logaritmo da função de verossimilhança requer uma integração, sugerindo a utilização do método de aproximação da quadratura de Gauss-Hermite para contornar 
Tabela 2.4: Média, Viés e REQM das estimativas de Monte Carlo dos parâmetros considerando três réplicas para $w_{t}$. Verdadeiros valores: $\alpha=2,0, \beta=-0,6, \gamma=2,5, \lambda=0,5, \mu_{x}=2,5, \sigma_{x}^{2}=2,7$ e $\sigma_{e}^{2}=0,05$. Cenário 2

\begin{tabular}{|c|c|c|c|c|c|c|}
\hline$n$ & Método & & $\alpha$ & $\beta$ & $\gamma$ & $\lambda$ \\
\hline \multirow{12}{*}{25} & \multirow{3}{*}{$\ell_{a}$} & Média & 2,00 & $-0,60$ & 2,50 & 0,59 \\
\hline & & Viés & 0,00 & 0,00 & 0,00 & 0,09 \\
\hline & & REQM & 0,19 & 0,05 & 0,28 & 0,23 \\
\hline & \multirow{3}{*}{$\ell_{p}$} & Média & 2,00 & $-0,60$ & 2,50 & 0,59 \\
\hline & & Viés & 0,00 & 0,00 & 0,00 & 0,09 \\
\hline & & REQM & 0,19 & 0,05 & 0,27 & 0,23 \\
\hline & \multirow{3}{*}{$\ell_{R C}$} & Média & 1,99 & $-0,59$ & 2,47 & 0,48 \\
\hline & & Viés & $-0,01$ & 0,01 & $-0,03$ & $-0,02$ \\
\hline & & REQM & 0,44 & 0,17 & 0,45 & 0,21 \\
\hline & \multirow{3}{*}{$\ell_{\text {Naïve }}$} & Média & 1,97 & $-0,58$ & 2,49 & 0,45 \\
\hline & & Viés & $-0,03$ & 0,02 & $-0,01$ & $-0,05$ \\
\hline & & REQM & 0,18 & 0,05 & 0,29 & 0,21 \\
\hline \multirow{12}{*}{50} & \multirow{3}{*}{$\ell_{a}$} & Média & 2,00 & $-0,60$ & 2,51 & 0,54 \\
\hline & & Viés & 0,00 & 0,00 & 0,01 & 0,04 \\
\hline & & REQM & 0,13 & 0,03 & 0,26 & 0,14 \\
\hline & \multirow{3}{*}{$\ell_{p}$} & Média & 2,00 & $-0,60$ & 2,51 & 0,54 \\
\hline & & Viés & 0,00 & 0,00 & 0,01 & 0,04 \\
\hline & & REQM & 0,13 & 0,03 & 0,26 & 0,14 \\
\hline & \multirow{3}{*}{$\ell_{R C}$} & Média & 1,98 & $-0,59$ & 2,49 & 0,45 \\
\hline & & Viés & $-0,02$ & 0,01 & $-0,01$ & $-0,05$ \\
\hline & & REQM & 0,33 & 0,12 & 0,33 & 0,16 \\
\hline & \multirow{3}{*}{$\ell_{\text {Nä̈ve }}$} & Média & 1,97 & $-0,59$ & 2,50 & 0,43 \\
\hline & & Viés & $-0,03$ & 0,01 & 0,00 & $-0,07$ \\
\hline & & REQM & 0,13 & 0,04 & 0,25 & 0,17 \\
\hline \multirow{12}{*}{100} & \multirow{3}{*}{$\ell_{a}$} & Média & 2,00 & $-0,60$ & 2,51 & 0,52 \\
\hline & & Viés & 0,00 & 0,00 & 0,01 & 0,02 \\
\hline & & REQM & 0,09 & 0,02 & 0,23 & 0,10 \\
\hline & \multirow{3}{*}{$\ell_{p}$} & Média & 2,00 & $-0,60$ & 2,51 & 0,52 \\
\hline & & Viés & 0,00 & 0,00 & 0,01 & 0,02 \\
\hline & & REQM & 0,09 & 0,02 & 0,23 & 0,10 \\
\hline & \multirow{3}{*}{$\ell_{R C}$} & Média & 1,99 & $-0,59$ & 2,49 & 0,43 \\
\hline & & Viés & $-0,01$ & 0,01 & $-0,01$ & $-0,07$ \\
\hline & & REQM & 0,24 & 0,09 & 0,27 & 0,14 \\
\hline & \multirow{3}{*}{$\ell_{\text {Naive }}$} & Média & 1,97 & $-0,59$ & 2,50 & 0,42 \\
\hline & & Viés & $-0,03$ & 0,01 & 0,00 & $-0,08$ \\
\hline & & REQM & 0,09 & 0,03 & 0,21 & 0,15 \\
\hline
\end{tabular}


Tabela 2.5: Média, Viés e REQM das estimativas de Monte Carlo dos parâmetros considerando três réplicas para $w_{t}$. Verdadeiros valores: $\alpha=2,0, \beta=-0,6, \gamma=2,5, \lambda=0,5, \mu_{x}=2,5, \sigma_{x}^{2}=2,7$ e $\sigma_{e}^{2}=0,50$. Cenário 2

\begin{tabular}{|c|c|c|c|c|c|c|}
\hline$n$ & Método & & $\alpha$ & $\beta$ & $\gamma$ & $\lambda$ \\
\hline \multirow{12}{*}{25} & \multirow{3}{*}{$\ell_{a}$} & Média & 1,99 & $-0,59$ & 2,47 & 0,68 \\
\hline & & Viés & $-0,01$ & 0,01 & $-0,03$ & 0,18 \\
\hline & & REQM & 0,29 & 0,09 & 0,39 & 0,40 \\
\hline & \multirow{3}{*}{$\ell_{p}$} & Média & 1,96 & $-0,59$ & 2,50 & 0,66 \\
\hline & & Viés & $-0,04$ & 0,01 & 0,00 & 0,16 \\
\hline & & REQM & 0,31 & 0,09 & 0,28 & 0,37 \\
\hline & \multirow{3}{*}{$\ell_{R C}$} & Média & 1,95 & $-0,58$ & 2,17 & 0,25 \\
\hline & & Viés & $-0,05$ & 0,02 & $-0,33$ & $-0,25$ \\
\hline & & REQM & 0,47 & 0,17 & 0,58 & 0,36 \\
\hline & \multirow{3}{*}{$\ell_{\text {Naïve }}$} & Média & 1,71 & $-0,49$ & 2,29 & 0,21 \\
\hline & & Viés & $-0,29$ & 0,11 & $-0,21$ & $-0,29$ \\
\hline & & REQM & 0,37 & 0,13 & 0,40 & 0,34 \\
\hline \multirow{12}{*}{50} & \multirow{3}{*}{$\ell_{a}$} & Média & 1,99 & $-0,60$ & 2,49 & 0,59 \\
\hline & & Viés & $-0,01$ & 0,00 & $-0,01$ & 0,09 \\
\hline & & REQM & 0,20 & 0,06 & 0,29 & 0,27 \\
\hline & \multirow{3}{*}{$\ell_{p}$} & Média & 1,98 & $-0,60$ & 2,50 & 0,58 \\
\hline & & Viés & $-0,02$ & 0,00 & 0,00 & 0,08 \\
\hline & & REQM & 0,21 & 0,06 & 0,27 & 0,25 \\
\hline & \multirow{3}{*}{$\ell_{R C}$} & Média & 1,95 & $-0,58$ & 2,16 & 0,23 \\
\hline & & Viés & $-0,05$ & 0,02 & $-0,34$ & $-0,27$ \\
\hline & & REQM & 0,34 & 0,13 & 0,47 & 0,33 \\
\hline & \multirow{3}{*}{$\ell_{\text {Naïve }}$} & Média & 1,72 & $-0,49$ & 2,27 & 0,20 \\
\hline & & Viés & $-0,28$ & 0,11 & $-0,23$ & $-0,30$ \\
\hline & & REQM & 0,32 & 0,12 & 0,35 & 0,32 \\
\hline \multirow{12}{*}{100} & \multirow{3}{*}{$\ell_{a}$} & Média & 2,00 & $-0,60$ & 2,50 & 0,55 \\
\hline & & Viés & 0,00 & 0,00 & 0,00 & 0,05 \\
\hline & & REQM & 0,14 & 0,04 & 0,26 & 0,19 \\
\hline & \multirow{3}{*}{$\ell_{p}$} & Média & 1,99 & $-0,60$ & 2,51 & 0,55 \\
\hline & & Viés & $-0,01$ & 0,00 & 0,01 & 0,05 \\
\hline & & REQM & 0,15 & 0,04 & 0,25 & 0,19 \\
\hline & \multirow{3}{*}{$\ell_{R C}$} & Média & 1,95 & $-0,58$ & 2,16 & 0,23 \\
\hline & & Viés & $-0,05$ & 0,02 & $-0,34$ & $-0,27$ \\
\hline & & REQM & 0,24 & 0,09 & 0,43 & 0,30 \\
\hline & \multirow{3}{*}{$\ell_{\text {Naïve }}$} & Média & 1,72 & $-0,49$ & 2,26 & 0,21 \\
\hline & & Viés & $-0,28$ & 0,11 & $-0,24$ & $-0,29$ \\
\hline & & REQM & 0,30 & 0,11 & 0,32 & 0,31 \\
\hline
\end{tabular}


Tabela 2.6: Média, Viés e REQM das estimativas de Monte Carlo dos parâmetros considerando três réplicas para $w_{t}$. Verdadeiros valores: $\alpha=2,0, \beta=-0,6, \gamma=2,5, \lambda=0,5, \mu_{x}=2,5, \sigma_{x}^{2}=2,7$ e $\sigma_{e}^{2}=1,0$. Cenário 2

\begin{tabular}{|c|c|c|c|c|c|c|}
\hline$n$ & Método & & $\alpha$ & $\beta$ & $\gamma$ & $\lambda$ \\
\hline \multirow{12}{*}{25} & \multirow{3}{*}{$\ell_{a}$} & Média & 1,99 & $-0,59$ & 2,47 & 0,68 \\
\hline & & Viés & $-0,01$ & 0,01 & $-0,03$ & 0,18 \\
\hline & & REQM & 0,37 & 0,12 & 0,55 & 0,41 \\
\hline & \multirow{3}{*}{$\ell_{p}$} & Média & 1,96 & $-0,59$ & 2,48 & 0,64 \\
\hline & & Viés & $-0,04$ & 0,01 & $-0,02$ & 0,14 \\
\hline & & REQM & 0,38 & 0,12 & 0,28 & 0,34 \\
\hline & \multirow{3}{*}{$\ell_{R C}$} & Média & 1,92 & $-0,57$ & 1,96 & 0,19 \\
\hline & & Viés & $-0,08$ & 0,03 & $-0,54$ & $-0,31$ \\
\hline & & REQM & 0,50 & 0,18 & 0,74 & 0,41 \\
\hline & \multirow{3}{*}{$\ell_{\text {Naïve }}$} & Média & 1,52 & $-0,41$ & 2,14 & 0,15 \\
\hline & & Viés & $-0,48$ & 0,19 & $-0,36$ & $-0,35$ \\
\hline & & REQM & 0,54 & 0,20 & 0,52 & 0,38 \\
\hline \multirow{12}{*}{50} & \multirow{3}{*}{$\ell_{a}$} & Média & 1,98 & $-0,59$ & 2,48 & 0,57 \\
\hline & & Viés & $-0,02$ & 0,01 & $-0,02$ & 0,07 \\
\hline & & REQM & 0,25 & 0,08 & 0,32 & 0,25 \\
\hline & \multirow{3}{*}{$\ell_{p}$} & Média & 1,96 & $-0,59$ & 2,49 & 0,56 \\
\hline & & Viés & $-0,04$ & 0,01 & $-0,01$ & 0,06 \\
\hline & & REQM & 0,27 & 0,08 & 0,27 & 0,24 \\
\hline & \multirow{3}{*}{$\ell_{R C}$} & Média & 1,91 & $-0,57$ & 1,95 & 0,16 \\
\hline & & Viés & $-0,09$ & 0,03 & $-0,55$ & $-0,34$ \\
\hline & & REQM & 0,36 & 0,13 & 0,66 & 0,40 \\
\hline & \multirow{3}{*}{$\ell_{\text {Naïve }}$} & Média & 1,52 & $-0,41$ & 2,10 & 0,14 \\
\hline & & Viés & $-0,48$ & 0,19 & $-0,40$ & $-0,36$ \\
\hline & & REQM & 0,51 & 0,19 & 0,48 & 0,38 \\
\hline \multirow{12}{*}{100} & \multirow{3}{*}{$\ell_{a}$} & Média & 1,99 & $-0,59$ & 2,49 & 0,52 \\
\hline & & Viés & $-0,01$ & 0,01 & $-0,01$ & 0,02 \\
\hline & & REQM & 0,17 & 0,06 & 0,27 & 0,18 \\
\hline & \multirow{3}{*}{$\ell_{p}$} & Média & 1,98 & $-0,59$ & 2,50 & 0,52 \\
\hline & & Viés & $-0,02$ & 0,01 & 0,00 & 0,02 \\
\hline & & REQM & 0,19 & 0,06 & 0,26 & 0,18 \\
\hline & \multirow{3}{*}{$\ell_{R C}$} & Média & 1,91 & $-0,57$ & 1,93 & 0,13 \\
\hline & & Viés & $-0,09$ & 0,03 & $-0,57$ & $-0,37$ \\
\hline & & REQM & 0,26 & 0,10 & 0,63 & 0,42 \\
\hline & \multirow{3}{*}{$\ell_{\text {Naive }}$} & Média & 1,53 & $-0,42$ & 2,07 & 0,14 \\
\hline & & Viés & $-0,47$ & 0,18 & $-0,43$ & $-0,36$ \\
\hline & & REQM & 0,49 & 0,18 & 0,48 & 0,37 \\
\hline
\end{tabular}


Tabela 2.7: Média, Viés e REQM das estimativas de Monte Carlo dos parâmetros com variância do erro de medida $\sigma_{e}^{2}=0,05$ e duas covariáveis medidas com erro. Verdadeiros valores: $\alpha=2,0, \beta_{1}=-0,6, \beta_{2}=-1,0$, $\gamma=2,5, \lambda=0,5, \mu_{x 1}=\mu_{x 2}=2,5$ e $\sigma_{x 1}^{2}=\sigma_{x 2}^{2}=2,7$. Cenário 3

\begin{tabular}{|c|c|c|c|c|c|c|c|}
\hline$n$ & Método & & $\alpha$ & $\beta_{1}$ & $\beta_{2}$ & $\gamma$ & $\lambda$ \\
\hline \multirow{12}{*}{25} & \multirow{3}{*}{$\ell_{a}$} & Média & 1,97 & $-0,59$ & $-0,99$ & 2,51 & 0,59 \\
\hline & & Viés & $-0,03$ & 0,01 & 0,01 & 0,01 & 0,09 \\
\hline & & REQM & 0,33 & 0,08 & 0,10 & 0,28 & 0,24 \\
\hline & \multirow{3}{*}{$\ell_{p}$} & Média & 1,97 & $-0,59$ & $-0,99$ & 2,51 & 0,59 \\
\hline & & Viés & $-0,03$ & 0,01 & 0,01 & 0,01 & 0,09 \\
\hline & & REQM & 0,33 & 0,08 & 0,09 & 0,28 & 0,23 \\
\hline & \multirow{3}{*}{$\ell_{R C}$} & Média & 1,82 & $-0,58$ & $-0,93$ & 2,30 & 0,51 \\
\hline & & Viés & $-0,18$ & 0,02 & 0,07 & $-0,20$ & 0,01 \\
\hline & & REQM & 0,86 & 0,18 & 0,29 & 0,51 & 0,28 \\
\hline & \multirow{3}{*}{$\ell_{\text {Naïve }}$} & Média & 1,86 & $-0,58$ & $-0,95$ & 2,31 & 0,53 \\
\hline & & Viés & $-0,14$ & 0,02 & 0,05 & $-0,19$ & 0,03 \\
\hline & & REQM & 0,35 & 0,08 & 0,10 & 0,36 & 0,18 \\
\hline \multirow{12}{*}{50} & \multirow{3}{*}{$\ell_{a}$} & Média & 1,97 & $-0,59$ & $-0,99$ & 2,50 & 0,55 \\
\hline & & Viés & $-0,03$ & 0,01 & 0,01 & 0,00 & 0,05 \\
\hline & & REQM & 0,22 & 0,05 & 0,06 & 0,26 & 0,14 \\
\hline & \multirow{3}{*}{$\ell_{p}$} & Média & 1,97 & $-0,59$ & $-0,99$ & 2,50 & 0,55 \\
\hline & & Viés & $-0,03$ & 0,01 & 0,01 & 0,00 & 0,05 \\
\hline & & REQM & 0,22 & 0,06 & 0,06 & 0,26 & 0,14 \\
\hline & \multirow{3}{*}{$\ell_{R C}$} & Média & 1,85 & $-0,58$ & $-0,94$ & 2,32 & 0,48 \\
\hline & & Viés & $-0,15$ & 0,02 & 0,06 & $-0,18$ & $-0,02$ \\
\hline & & REQM & 0,61 & 0,13 & 0,21 & 0,39 & 0,17 \\
\hline & \multirow{3}{*}{$\ell_{\text {Naïve }}$} & Média & 1,85 & $-0,57$ & $-0,95$ & 2,33 & 0,48 \\
\hline & & Viés & $-0,15$ & 0,03 & 0,05 & $-0,17$ & $-0,02$ \\
\hline & & REQM & 0,26 & 0,06 & 0,08 & 0,30 & 0,13 \\
\hline \multirow{12}{*}{100} & \multirow{3}{*}{$\ell_{a}$} & Média & 1,97 & $-0,59$ & $-0,99$ & 2,50 & 0,53 \\
\hline & & Viés & $-0,03$ & 0,01 & 0,01 & 0,00 & 0,03 \\
\hline & & REQM & 0,15 & 0,04 & 0,05 & 0,24 & 0,10 \\
\hline & \multirow{3}{*}{$\ell_{p}$} & Média & 1,97 & $-0,59$ & $-0,99$ & 2,50 & 0,53 \\
\hline & & Viés & $-0,03$ & 0,01 & 0,01 & 0,00 & 0,03 \\
\hline & & REQM & 0,15 & 0,04 & 0,05 & 0,24 & 0,10 \\
\hline & \multirow{3}{*}{$\ell_{R C}$} & Média & 1,88 & $-0,58$ & $-0,95$ & 2,33 & 0,46 \\
\hline & & Viés & $-0,12$ & 0,02 & 0,05 & $-0,17$ & $-0,04$ \\
\hline & & REQM & 0,44 & 0,09 & 0,15 & 0,32 & 0,13 \\
\hline & \multirow{3}{*}{$\ell_{\text {Naïve }}$} & Média & 1.85 & -0.57 & -0.94 & 2.34 & 0.46 \\
\hline & & Viés & -0.15 & 0.03 & 0.06 & -0.16 & -0.04 \\
\hline & & REQM & 0.20 & 0.05 & 0.07 & 0.27 & 0.12 \\
\hline
\end{tabular}


Tabela 2.8: Média, Viés e REQM das estimativas de Monte Carlo dos parâmetros com variância do erro de medida $\sigma_{e}^{2}=0,50$ e duas covariáveis medidas com erro. Verdadeiros valores: $\alpha=2,0, \beta_{1}=-0,6, \beta_{2}=-1,0$, $\gamma=2,5, \lambda=0,5, \mu_{x 1}=\mu_{x 2}=2,5$ e $\sigma_{x 1}^{2}=\sigma_{x 2}^{2}=2,7$. Cenário 3

\begin{tabular}{|c|c|c|c|c|c|c|c|}
\hline$n$ & Método & & $\alpha$ & $\beta_{1}$ & $\beta_{2}$ & $\gamma$ & $\lambda$ \\
\hline \multirow{12}{*}{25} & \multirow{3}{*}{$\ell_{a}$} & Média & 1,96 & $-0,59$ & $-1,00$ & 2,48 & 0,92 \\
\hline & & Viés & $-0,04$ & 0,01 & 0,00 & $-0,02$ & 0,42 \\
\hline & & REQM & 0,65 & 0,16 & 0,19 & 1,04 & 0,97 \\
\hline & \multirow{3}{*}{$\ell_{p}$} & Média & 1,96 & $-0,59$ & $-1,00$ & 2,49 & 0,86 \\
\hline & & Viés & $-0,04$ & 0,01 & 0,00 & $-0,01$ & 0,36 \\
\hline & & REQM & 0,63 & 0,16 & 0,19 & 0,28 & 0,73 \\
\hline & \multirow{3}{*}{$\ell_{R C}$} & Média & 1,61 & $-0,53$ & $-0,81$ & 1,58 & 0,32 \\
\hline & & Viés & $-0,39$ & 0,07 & 0,19 & $-0,92$ & $-0,18$ \\
\hline & & REQM & 0,95 & 0,21 & 0,32 & 1,08 & 0,39 \\
\hline & \multirow{3}{*}{$\ell_{\text {Naïve }}$} & Média & 1,12 & $-0,44$ & $-0,71$ & 1,75 & 0,29 \\
\hline & & Viés & $-0,88$ & 0,16 & 0,29 & $-0,75$ & $-0,21$ \\
\hline & & REQM & 0,99 & 0,19 & 0,32 & 0,87 & 0,33 \\
\hline \multirow{12}{*}{50} & \multirow{3}{*}{$\ell_{a}$} & Média & 1,99 & $-0,59$ & $-1,00$ & 2,48 & 0,72 \\
\hline & & Viés & $-0,01$ & 0,01 & 0,00 & $-0,02$ & 0,22 \\
\hline & & REQM & 0,42 & 0,11 & 0,13 & 0,48 & 0,49 \\
\hline & \multirow{3}{*}{$\ell_{p}$} & Média & 1,98 & $-0,59$ & $-1,00$ & 2,48 & 0,71 \\
\hline & & Viés & $-0,02$ & 0,01 & 0,00 & $-0,02$ & 0,21 \\
\hline & & REQM & 0,42 & 0,11 & 0,13 & 0,28 & 0,44 \\
\hline & \multirow{3}{*}{$\ell_{R C}$} & Média & 1,62 & $-0,53$ & $-0,81$ & 1,52 & 0,31 \\
\hline & & Viés & $-0,38$ & 0,07 & 0,19 & $-0,98$ & $-0,19$ \\
\hline & & REQM & 0,71 & 0,16 & 0,27 & 1,06 & 0,32 \\
\hline & \multirow{3}{*}{$\ell_{\text {Naïve }}$} & Média & 1,12 & $-0,44$ & $-0,69$ & 1,66 & 0,26 \\
\hline & & Viés & $-0,88$ & 0,16 & 0,31 & $-0,84$ & $-0,24$ \\
\hline & & REQM & 0,93 & 0,18 & 0,31 & 0,89 & 0,32 \\
\hline \multirow{12}{*}{100} & \multirow{3}{*}{$\ell_{a}$} & Média & 1,99 & $-0,59$ & $-1,00$ & 2,49 & 0,62 \\
\hline & & Viés & $-0,01$ & 0,01 & 0,00 & $-0,01$ & 0,12 \\
\hline & & REQM & 0,29 & 0,07 & 0,09 & 0,29 & 0,26 \\
\hline & \multirow{3}{*}{$\ell_{p}$} & Média & 1,99 & $-0,59$ & $-0,99$ & 2,49 & 0,62 \\
\hline & & Viés & $-0,01$ & 0,01 & 0,01 & $-0,01$ & 0,12 \\
\hline & & REQM & 0,28 & 0,07 & 0,09 & 0,27 & 0,25 \\
\hline & \multirow{3}{*}{$\ell_{R C}$} & Média & 1,62 & $-0,52$ & $-0,81$ & 1,49 & 0,28 \\
\hline & & Viés & $-0,38$ & 0,08 & 0,19 & $-1,01$ & $-0,22$ \\
\hline & & REQM & 0,56 & 0,13 & 0,23 & 1,05 & 0,33 \\
\hline & \multirow{3}{*}{$\ell_{\text {Naïve }}$} & Média & 1,12 & $-0,44$ & $-0,69$ & 1,62 & 0,24 \\
\hline & & Viés & $-0,88$ & 0,16 & 0,31 & $-0,88$ & $-0,26$ \\
\hline & & REQM & 0,90 & 0,17 & 0,32 & 0,90 & 0,32 \\
\hline
\end{tabular}


Tabela 2.9: Média, Viés e REQM das estimativas de Monte Carlo dos parâmetros com variância do erro de medida $\sigma_{e}^{2}=1,0$ e duas covariáveis medidas com erro. Verdadeiros valores: $\alpha=2,0, \beta_{1}=-0,6, \beta_{2}=-1,0$, $\gamma=2,5, \lambda=0,5, \mu_{x 1}=\mu_{x 2}=2,5$ e $\sigma_{x 1}^{2}=\sigma_{x 2}^{2}=2,7$. Cenário 3

\begin{tabular}{|c|c|c|c|c|c|c|c|}
\hline$n$ & Método & & $\alpha$ & $\beta_{1}$ & $\beta_{2}$ & $\gamma$ & $\lambda$ \\
\hline \multirow{12}{*}{25} & \multirow{3}{*}{$\ell_{a}$} & Média & 1,94 & $-0,58$ & $-1,00$ & 2,48 & 1,07 \\
\hline & & Viés & $-0,06$ & 0,02 & 0,00 & $-0,02$ & 0,57 \\
\hline & & REQM & 0,82 & 0,23 & 0,25 & 1,53 & 1,20 \\
\hline & \multirow{3}{*}{$\ell_{p}$} & Média & 1,96 & $-0,58$ & $-1,01$ & 2,50 & 0,93 \\
\hline & & Viés & $-0,04$ & 0,02 & $-0,01$ & 0,00 & 0,43 \\
\hline & & REQM & 0,83 & 0,22 & 0,26 & 0,29 & 0,81 \\
\hline & \multirow{3}{*}{$\ell_{R C}$} & Média & 1,47 & $-0,50$ & $-0,75$ & 1,28 & 0,27 \\
\hline & & Viés & $-0,53$ & 0,10 & 0,25 & $-1,22$ & $-0,23$ \\
\hline & & REQM & 1,02 & 0,24 & 0,36 & 1,36 & 0,42 \\
\hline & \multirow{3}{*}{$\ell_{\text {Naïve }}$} & Média & 0,66 & $-0,36$ & $-0,57$ & 1,51 & 0,20 \\
\hline & & Viés & $-1,34$ & 0,24 & 0,43 & $-0,99$ & $-0,30$ \\
\hline & & REQM & 1,41 & 0,26 & 0,45 & 1,09 & 0,38 \\
\hline \multirow{12}{*}{50} & \multirow{3}{*}{$\ell_{a}$} & Média & 2,02 & $-0,60$ & $-1,01$ & 2,50 & 0,85 \\
\hline & & Viés & 0,02 & 0,00 & $-0,01$ & 0,00 & 0,35 \\
\hline & & REQM & 0,59 & 0,15 & 0,18 & 0,85 & 0,68 \\
\hline & \multirow{3}{*}{$\ell_{p}$} & Média & 2,01 & $-0,60$ & $-1,01$ & 2,48 & 0,81 \\
\hline & & Viés & 0,01 & 0,00 & $-0,01$ & $-0,02$ & 0,31 \\
\hline & & REQM & 0,56 & 0,15 & 0,17 & 0,28 & 0,54 \\
\hline & \multirow{3}{*}{$\ell_{R C}$} & Média & 1,50 & $-0,50$ & $-0,75$ & 1,16 & 0,26 \\
\hline & & Viés & $-0,50$ & 0,10 & 0,25 & $-1,34$ & $-0,24$ \\
\hline & & REQM & 0,79 & 0,18 & 0,31 & 1,41 & 0,36 \\
\hline & \multirow{3}{*}{$\ell_{\text {Naïve }}$} & Média & 0,66 & $-0,36$ & $-0,55$ & 1,38 & 0,19 \\
\hline & & Viés & $-1,34$ & 0,24 & 0,45 & $-1,12$ & $-0,31$ \\
\hline & & REQM & 1,38 & 0,25 & 0,45 & 1,16 & 0,35 \\
\hline \multirow{12}{*}{100} & \multirow{3}{*}{$\ell_{a}$} & Média & 2,03 & $-0,60$ & $-1,01$ & 2,48 & 0,71 \\
\hline & & Viés & 0,03 & 0,00 & $-0,01$ & $-0,02$ & 0,21 \\
\hline & & REQM & 0,39 & 0,10 & 0,12 & 0,41 & 0,39 \\
\hline & \multirow{3}{*}{$\ell_{p}$} & Média & 2,01 & $-0,60$ & $-1,01$ & 2,48 & 0,68 \\
\hline & & Viés & 0,01 & 0,00 & $-0,01$ & $-0,02$ & 0,18 \\
\hline & & REQM & 0,37 & 0,09 & 0,11 & 0,27 & 0,34 \\
\hline & \multirow{3}{*}{$\ell_{R C}$} & Média & 1,49 & $-0,49$ & $-0,74$ & 1,10 & 0,25 \\
\hline & & Viés & $-0,51$ & 0,11 & 0,26 & $-1,40$ & $-0,25$ \\
\hline & & REQM & 0,66 & 0,15 & 0,29 & 1,44 & 0,34 \\
\hline & \multirow{3}{*}{$\ell_{\text {Naïve }}$} & Média & 0,66 & $-0,36$ & $-0,54$ & 1,33 & 0,19 \\
\hline & & Viés & $-1,34$ & 0,24 & 0,46 & $-1,17$ & $-0,31$ \\
\hline & & REQM & 1,36 & 0,25 & 0,46 & 1,20 & 0,35 \\
\hline
\end{tabular}


esse fato na estimação de parâmetros. Os métodos de estimação dos parâmetros, que precisam de aproximações numéricas para estimar os parâmetros são: máxima verossimilhança aproximada e pseudo-verossimilhança aproximada. Como todo método de aproximação, o método da quadratura de Gauss-Hermite utilizado neste trabalho apresenta algumas deficiências, entre elas a escolha adequada do número de pontos de quadratura. Assim, com o objetivo de avaliar a influência na escolha do número de pontos de quadratura, realizamos um estudo de simulação. Neste estudo, consideramos $Q=12,24$ e 48, pontos de quadratura. Assumimos que o modelo de regressão beta com erros de medida apresenta a mesma estrutura dada no cenário 1. Estimamos os parâmetros via máxima verossimilhança aproximada e pseudo-verossimilhança aproximada para cada réplica de Monte Carlo, e ao final obtemos estimativas da média, do viés e raiz do erro quadrático médio. As Tabelas 2.10, 2.11 e 2.12 apresentam os resultados neste cenário.

Notamos a partir destas tabelas que o desempenho dos estimadores dos parâmetros, em termos de viés e erro quadrático médio, melhora apenas levemente conforme o número de pontos de quadratura $Q$ aumenta. Deste modo, concluímos que neste cenário inferências no modelo de regressão beta com erros de medida parecem ser pouco afetadas pelo número de pontos de quadratura.

Do estudo de simulação realizado, concluímos que os métodos de estimação por máxima verossimilhança aproximada e pseudo-verossimilhança aproximada tiveram melhor desempenho relativamente aos outros dois (nä̈ve e calibração da regressão). Como os dois primeiros métodos apresentam resultados semelhantes, recomendamos a utilização do método de máxima pseudo-verossimilhança aproximada, pois é computacionalmente mais rápido, eficiente e o número de iterações até conseguir a convergência foi inferior ao do método de máxima verossimilhança aproximada.

Inicialmente foram utilizadas as linguagens de programação ox (versão 6.2), R (versão 2.3.7.1) e SAS (versão 9.2). Programas de estimação foram escritos utilizando rotinas de maximização como MaxSQPF em Ox, optim em R e nlmixed em SAS. Encontramos algumas deficiências computacionais, por exemplo, imprecisão e alto tempo da execução nas linguagens SAS e R, respectivamente. Recorde que simulamos 10000 réplicas de Monte Carlo e em cada observação da amostra gerada consideramos 12 pontos de quadratura para aproximar a integral. Assim, supondo um tamanho de amostra $n=100$ teremos um total de 1200 avaliações de quadraturas de Gauss-Hermite. Esta quantidade multiplicada pelo número de réplicas torna o programa extremamente impreciso e demorado (72 horas aproximadamente) na linguagem de programação SAS e R respectivamente. No entanto, a linguagem de programação $\mathrm{Ox}$ mostrou melhores resultados quanto à precisão e ao tempo de execução (duas horas aproximadamente).

Por essas razões, utilizamos a linguagem de programação ox como plataforma computacional para o estudo de simulação e, posteriormente, para os casos práticos. 
Tabela 2.10: Média, Viés e REQM das estimativas de Monte Carlo dos parâmetros com variância do erro de medida $\sigma_{e}^{2}=0,05$. Verdadeiros valores: $\alpha=2,0, \beta=-0,6, \gamma=2,5, \lambda=0,5, \mu_{x}=2,5$ e $\sigma_{x}^{2}=2,7$. Cenário 4

\begin{tabular}{|c|c|c|c|c|c|c|c|}
\hline $\bar{n}$ & $\overline{Q Q}$ & Método & & $\overline{\alpha \alpha}$ & $\bar{\beta}$ & $\overline{\bar{c} \gamma}$ & $\overline{\bar{\lambda}}$ \\
\hline \multirow{18}{*}{25} & \multirow{6}{*}{12} & \multirow{3}{*}{$\ell_{a}$} & Média & 1,92 & $-0,60$ & 2,56 & 0,53 \\
\hline & & & Viés & $-0,08$ & 0,00 & 0,06 & 0,03 \\
\hline & & & REQM & 0,61 & 0,06 & 0,86 & 0,35 \\
\hline & & \multirow{3}{*}{$\ell_{p}$} & Média & 1,94 & $-0,60$ & 2,58 & 0,54 \\
\hline & & & Viés & $-0,06$ & 0,00 & 0,08 & 0,04 \\
\hline & & & REQM & 0,55 & 0,06 & 0,82 & 0,35 \\
\hline & \multirow{6}{*}{24} & \multirow{3}{*}{$\ell_{a}$} & Média & 1,92 & $-0,60$ & 2,58 & 0,53 \\
\hline & & & Viés & $-0,08$ & 0,00 & 0,08 & 0,03 \\
\hline & & & REQM & 0,59 & 0,06 & 0,81 & 0,36 \\
\hline & & \multirow{3}{*}{$\ell_{p}$} & Média & 1,95 & $-0,59$ & 2,59 & 0,54 \\
\hline & & & Viés & $-0,05$ & 0,01 & 0,09 & 0,04 \\
\hline & & & REQM & 0,49 & 0,18 & 0,78 & 0,34 \\
\hline & \multirow{6}{*}{48} & & Média & 1,94 & $-0,60$ & 2,58 & 0,53 \\
\hline & & \multirow[t]{2}{*}{$\ell_{a}$} & Viés & $-0,06$ & 0,00 & 0,08 & 0,03 \\
\hline & & & REQM & 0,53 & 0,05 & 0,83 & 0,34 \\
\hline & & \multirow{3}{*}{$\ell_{p}$} & Média & 1,94 & $-0,60$ & 2,61 & 0,52 \\
\hline & & & Viés & 0,06 & 0,00 & 0,11 & 0,02 \\
\hline & & & REQM & 0,53 & 0,06 & 0,76 & 0,34 \\
\hline \multirow{18}{*}{50} & \multirow{6}{*}{12} & \multirow{3}{*}{$\ell_{a}$} & Média & 1,89 & $-0,59$ & 2,50 & 0,49 \\
\hline & & & Viés & $-0,11$ & 0,01 & 0,00 & $-0,01$ \\
\hline & & & REQM & 0,67 & 0,18 & 0,69 & 0,24 \\
\hline & & & Média & 1,88 & $-0,60$ & 2,52 & 0,49 \\
\hline & & \multirow[t]{2}{*}{$\ell_{p}$} & Viés & $-0,12$ & 0,00 & 0,02 & $-0,01$ \\
\hline & & & REQM & 0,69 & 0,04 & 0,63 & 0,25 \\
\hline & \multirow{6}{*}{24} & \multirow{3}{*}{$\ell_{a}$} & Média & 1,92 & $-0,60$ & 2,54 & 0,51 \\
\hline & & & Viés & $-0,08$ & 0,00 & 0,04 & 0,01 \\
\hline & & & REQM & 0,55 & 0,04 & 0,55 & 0,21 \\
\hline & & \multirow{3}{*}{$\ell_{p}$} & Média & 1,94 & $-0,60$ & 2,55 & 0,50 \\
\hline & & & Viés & $-0,06$ & 0,00 & 0,05 & 0,00 \\
\hline & & & REQM & 0,48 & 0,04 & 0,47 & 0,20 \\
\hline & & \multirow{3}{*}{$\ell_{a}$} & Média & 1,93 & $-0,60$ & 2,54 & 0,50 \\
\hline & & & Viés & $-0,07$ & 0,00 & 0,04 & 0,00 \\
\hline & & & REQM & 0,52 & 0,03 & 0,54 & 0,21 \\
\hline & 48 & & Média & 1,92 & $-0,60$ & 2,53 & 0,50 \\
\hline & & $\ell_{p}$ & Viés & $-0,08$ & 0,00 & 0,03 & 0,00 \\
\hline & & & REQM & 0,51 & 0,02 & 0,43 & 0,10 \\
\hline & & & Média & 1,88 & $-0,60$ & 2,47 & 0,48 \\
\hline & & $\ell_{a}$ & Viés & $-0,12$ & 0,00 & $-0,03$ & $-0,02$ \\
\hline & & & REQM & 0,70 & 0,02 & 0,61 & 0,19 \\
\hline & 12 & & Média & 1,91 & $-0,59$ & 2,59 & 0,47 \\
\hline & & $\ell_{p}$ & Viés & $-0,09$ & 0,01 & $-0,01$ & $-0,03$ \\
\hline & & & REQM & 0,61 & 0,18 & 0,55 & 0,21 \\
\hline & & & Média & 1,94 & $-0,60$ & 2,50 & 0,49 \\
\hline & & $\ell_{a}$ & Viés & $-0,06$ & 0,00 & 0,00 & $-0,01$ \\
\hline 100 & & & REQM & 0,51 & 0,02 & 0,47 & 0,19 \\
\hline & 24 & & Média & 1,94 & $-0,60$ & 2,50 & 0,48 \\
\hline & & $\ell_{p}$ & Viés & $-0,06$ & 0,00 & 0,01 & $-0,02$ \\
\hline & & & REQM & 0,52 & 0,02 & 0,47 & 0,19 \\
\hline & & & Média & 1,94 & $-0,60$ & 2,49 & 0,49 \\
\hline & & $\ell_{a}$ & Viés & $-0,06$ & 0,00 & $-0,01$ & $-0,01$ \\
\hline & & & REQM & 0,52 & 0,02 & 0,54 & 0,18 \\
\hline & 48 & & Média & 1,94 & $-0,60$ & 2,51 & 0,48 \\
\hline & & $\ell_{p}$ & Viés & $-0,06$ & 0,00 & 0,01 & $-0,02$ \\
\hline & & & REQM & 0,52 & 0,02 & 0,43 & 0,20 \\
\hline
\end{tabular}


Tabela 2.11: Média, Viés e REQM das estimativas de Monte Carlo dos parâmetros com variância do erro de medida $\sigma_{e}^{2}=0,5$. Verdadeiros valores: $\alpha=2,0, \beta=-0,6, \gamma=2,5, \lambda=0,5, \mu_{x}=2,5$ e $\sigma_{x}^{2}=2,7$. Cenário 4

\begin{tabular}{|c|c|c|c|c|c|c|c|}
\hline$n$ & $\overline{Q Q}$ & Método & & $\overline{\alpha \alpha}$ & $\overline{\beta \beta}$ & $\gamma$ & $\overline{\bar{\lambda}}$ \\
\hline \multirow{18}{*}{25} & \multirow{6}{*}{12} & \multirow{3}{*}{$\ell_{a}$} & Média & 2,02 & $-0,61$ & 2,74 & 0,65 \\
\hline & & & Viés & 0,02 & $-0,01$ & 0,24 & 0,15 \\
\hline & & & REQM & 0,36 & 0,09 & 1,15 & 0,49 \\
\hline & & \multirow{3}{*}{$\ell_{p}$} & Média & 1,99 & $-0,61$ & 2,77 & 0,62 \\
\hline & & & Viés & $-0,01$ & $-0,01$ & 0,27 & 0,12 \\
\hline & & & REQM & 0,38 & 0,09 & 1,20 & 0,49 \\
\hline & \multirow{6}{*}{24} & \multirow{3}{*}{$\ell_{a}$} & Média & 2,01 & $-0,61$ & 2,71 & 0,65 \\
\hline & & & Viés & 0,01 & $-0,01$ & 0,21 & 0,15 \\
\hline & & & REQM & 0,39 & 0,10 & 1,20 & 0,49 \\
\hline & & \multirow{3}{*}{$\ell_{p}$} & Média & 2,00 & $-0,61$ & 2,76 & 0,66 \\
\hline & & & Viés & 0,00 & 0,00 & 0,24 & 0,16 \\
\hline & & & REQM & 0,40 & 0,10 & 1,20 & 0,50 \\
\hline & \multirow{6}{*}{48} & \multirow{3}{*}{$\ell_{a}$} & Média & 2,01 & $-0,61$ & 2,71 & 0,65 \\
\hline & & & Viés & 0,01 & $-0,01$ & 0,21 & 0,15 \\
\hline & & & REQM & 0,38 & 0,10 & 1,20 & 0,49 \\
\hline & & \multirow{3}{*}{$\ell_{p}$} & Média & 2,01 & $-0,61$ & 2,74 & 0,65 \\
\hline & & & Viés & 0,01 & 0,00 & 0,26 & 0,15 \\
\hline & & & REQM & 0,39 & 0,09 & 1,19 & 0,49 \\
\hline \multirow{18}{*}{50} & \multirow{6}{*}{12} & \multirow{3}{*}{$\ell_{a}$} & Média & 2,00 & $-0,60$ & 2,61 & 0,57 \\
\hline & & & Viés & 0,00 & 0,00 & 0,11 & 0,07 \\
\hline & & & REQM & 0,32 & 0,06 & 0,68 & 0,32 \\
\hline & & \multirow{3}{*}{$\ell_{p}$} & Média & 2,00 & $-0,60$ & 2,62 & 0,57 \\
\hline & & & Viés & 0,00 & 0,00 & 0,12 & 0,07 \\
\hline & & & REQM & 0,28 & 0,06 & 0,69 & 0,31 \\
\hline & \multirow{6}{*}{24} & \multirow{3}{*}{$\ell_{a}$} & Média & 2,00 & $-0,60$ & 2,59 & 0,52 \\
\hline & & & Viés & 0,00 & 0,00 & 0,09 & 0,02 \\
\hline & & & REQM & 0,33 & 0,07 & 0,70 & 0,30 \\
\hline & & \multirow{3}{*}{$\ell_{p}$} & Média & 1,99 & $-0,60$ & 2,60 & 0,52 \\
\hline & & & Viés & $-0,01$ & 0,00 & 0,10 & 0,02 \\
\hline & & & REQM & 0,30 & 0,06 & 0,69 & 0,29 \\
\hline & & \multirow{3}{*}{$\ell_{a}$} & Média & 2,00 & $-0,61$ & 2,59 & 0,51 \\
\hline & & & Viés & 0,00 & $-0,01$ & 0,09 & 0,01 \\
\hline & & & REQM & 0,33 & 0,07 & 0,69 & 0,28 \\
\hline & 48 & & Média & 2,01 & $-0,60$ & 2,60 & 0,52 \\
\hline & & $\ell_{p}$ & Viés & 0,01 & 0,00 & 0,10 & 0,02 \\
\hline & & & REQM & 0,29 & 0,06 & 0,63 & 0,29 \\
\hline & & & Média & 2,00 & $-0,60$ & 2,52 & 0,54 \\
\hline & & $\ell_{a}$ & Viés & 0,00 & 0,00 & 0,02 & 0,04 \\
\hline & & & REQM & 0,22 & 0,04 & 0,40 & 0,23 \\
\hline & 12 & & Média & 1,99 & $-0,60$ & 2,53 & 0,53 \\
\hline & & $\ell_{p}$ & Viés & $-0,01$ & 0,00 & 0,03 & 0,03 \\
\hline & & & REQM & 0,23 & 0,04 & 0,41 & 0,23 \\
\hline & & & Média & 1,99 & $-0,60$ & 2,49 & 0,52 \\
\hline & & $\ell_{a}$ & Viés & $-0,01$ & 0,00 & $-0,01$ & 0,02 \\
\hline 100 & & & REQM & 0,25 & 0,04 & 0,40 & 0,19 \\
\hline & 24 & & Média & 2,00 & $-0,60$ & 2,53 & 0,53 \\
\hline & & $\ell_{p}$ & Viés & 0,00 & 0,00 & 0,03 & 0,03 \\
\hline & & & REQM & 0,23 & 0,04 & 0,43 & 0,20 \\
\hline & & & Média & 2,00 & $-0,60$ & 2,51 & 0,51 \\
\hline & & $\ell_{a}$ & Viés & 0,00 & 0,00 & 0,01 & 0,01 \\
\hline & & & REQM & 0,19 & 0,04 & 0,42 & 0,19 \\
\hline & 48 & & Média & 2,00 & $-0,60$ & 2,51 & 0,52 \\
\hline & & $\ell_{p}$ & Viés & 0,00 & 0,00 & 0,01 & 0,02 \\
\hline & & & REQM & 0,20 & 0,02 & 0,43 & 0,19 \\
\hline
\end{tabular}


Tabela 2.12: Média, Viés e REQM das estimativas de Monte Carlo dos parâmetros com variância do erro de medida $\sigma_{e}^{2}=1,0$. Verdadeiros valores: $\alpha=2,0, \beta=-0,6, \gamma=2,5, \lambda=0,5, \mu_{x}=2,5$ e $\sigma_{x}^{2}=2,7$. Cenário 4

\begin{tabular}{|c|c|c|c|c|c|c|c|}
\hline$\overline{c n}$ & $\overline{Q Q}$ & Método & & $\overline{\alpha \alpha}$ & $\overline{\bar{\beta}}$ & $\bar{\gamma} \gamma$ & $\lambda$ \\
\hline \multirow{18}{*}{25} & \multirow{6}{*}{12} & \multirow{3}{*}{$\ell_{a}$} & Média & 2,00 & $-0,61$ & 2,68 & 0,60 \\
\hline & & & Viés & 0,00 & $-0,01$ & 0,18 & 0,10 \\
\hline & & & REQM & 0,59 & 0,14 & 1,14 & 0,52 \\
\hline & & \multirow{3}{*}{$\ell_{p}$} & Média & 1,99 & $-0,61$ & 2,68 & 0,56 \\
\hline & & & Viés & 0,00 & $-0,01$ & 0,18 & 0,06 \\
\hline & & & REQM & 0,46 & 0,13 & 1,13 & 0,49 \\
\hline & \multirow{6}{*}{24} & & Média & 2,00 & $-0,60$ & 2,69 & 0,57 \\
\hline & & \multirow[t]{2}{*}{$\ell_{a}$} & Viés & 0,00 & 0,00 & 0,19 & 0,07 \\
\hline & & & REQM & 0,59 & 0,15 & 1,18 & 0,42 \\
\hline & & \multirow{3}{*}{$\ell_{p}$} & Média & 2,00 & $-0,61$ & 2,65 & 0,51 \\
\hline & & & Viés & 0,00 & $-0,00$ & 0,15 & 0,01 \\
\hline & & & REQM & 0,46 & 0,13 & 1,17 & 0,41 \\
\hline & \multirow{6}{*}{48} & \multirow{3}{*}{$\ell_{a}$} & Média & 2,00 & $-0,60$ & 2,57 & 0,51 \\
\hline & & & Viés & 0,00 & 0,00 & 0,07 & 0,01 \\
\hline & & & REQM & 0,40 & 0,15 & 1,12 & 0,21 \\
\hline & & \multirow{3}{*}{$\ell_{p}$} & Média & 2,00 & $-0,60$ & 2,52 & 0,51 \\
\hline & & & Viés & 0,00 & 0,00 & 0,02 & 0,01 \\
\hline & & & REQM & 0,39 & 0,14 & 1,17 & 0,21 \\
\hline \multirow{18}{*}{50} & \multirow{6}{*}{12} & \multirow{3}{*}{$\ell_{a}$} & Média & 2,00 & $-0,60$ & 2,60 & 0,52 \\
\hline & & & Viés & 0,00 & 0,00 & 0,10 & 0,02 \\
\hline & & & REQM & 0,56 & 0,09 & 0,88 & 0,35 \\
\hline & & \multirow{3}{*}{$\ell_{p}$} & Média & 1,97 & $-0,60$ & 2,66 & 0,52 \\
\hline & & & Viés & $-0,03$ & 0,00 & 0,16 & 0,02 \\
\hline & & & REQM & 0,36 & 0,08 & 0,83 & 0,30 \\
\hline & \multirow{6}{*}{24} & \multirow{3}{*}{$\ell_{a}$} & Média & 2,00 & $-0,60$ & 2,53 & 0,51 \\
\hline & & & Viés & 0,00 & 0,00 & 0,03 & 0,01 \\
\hline & & & REQM & 0,49 & 0,10 & 0,83 & 0,33 \\
\hline & & \multirow{3}{*}{$\ell_{p}$} & Média & 2,00 & $-0,60$ & 2,53 & 0,51 \\
\hline & & & Viés & 0,00 & 0,00 & 0,03 & 0,01 \\
\hline & & & REQM & 0,29 & 0,08 & 0,73 & 0,29 \\
\hline & & \multirow{3}{*}{$\ell_{a}$} & Média & 2,00 & $-0,60$ & 2,51 & 0,51 \\
\hline & & & Viés & 0,00 & 0,00 & 0,01 & 0,02 \\
\hline & & & REQM & 0,49 & 0,02 & 0,62 & 0,30 \\
\hline & 48 & & Média & 2,00 & $-0,60$ & 2,51 & 0,52 \\
\hline & & $\ell_{p}$ & Viés & 0,00 & 0,00 & 0,01 & 0,02 \\
\hline & & & REQM & 0,09 & 0,02 & 0,63 & 0,20 \\
\hline & & & Média & 2,00 & $-0,60$ & 2,52 & 0,49 \\
\hline & & $\ell_{a}$ & Viés & 0,00 & 0,00 & 0,02 & $-0,01$ \\
\hline & & & REQM & 0,49 & 0,07 & 0,60 & 0,24 \\
\hline & 12 & & Média & 2,00 & $-0,60$ & 2,55 & 0,51 \\
\hline & & $\ell_{p}$ & Viés & 0,00 & 0,00 & 0,05 & 0,01 \\
\hline & & & REQM & 0,31 & 0,05 & 0,54 & 0,21 \\
\hline & & & Média & 2,00 & $-0,59$ & 2,52 & 0,48 \\
\hline & & $\ell_{a}$ & Viés & 0,00 & 0,00 & 0,02 & $-0,02$ \\
\hline 100 & & & REQM & 0,39 & 0,08 & 0,53 & 0,23 \\
\hline & 24 & & Média & 2,00 & $-0,60$ & 2,52 & 0,52 \\
\hline & & $\ell_{p}$ & Viés & 0,00 & 0,00 & 0,01 & 0,02 \\
\hline & & & REQM & 0,29 & 0,05 & 0,43 & 0,18 \\
\hline & & & Média & 2,02 & $-0,59$ & 2,49 & 0,51 \\
\hline & & $\ell_{a}$ & Viés & 0,02 & 0,01 & $-0,01$ & $-0,01$ \\
\hline & & & REQM & 0,30 & 0,02 & 0,52 & 0,20 \\
\hline & 48 & & Média & 1,99 & $-0,60$ & 2,51 & 0,52 \\
\hline & & $\ell_{p}$ & Viés & $-0,01$ & 0,00 & 0,01 & 0,02 \\
\hline & & & REQM & 0,29 & 0,04 & 0,41 & 0,20 \\
\hline
\end{tabular}




\section{Capítulo 3}

\section{Distribuições assintóticas e testes de hipóteses}

Propriedades assintóticas dos estimadores são necessárias para construir intervalos de confiança e testar hipóteses. Neste capítulo apresentamos distribuições assintóticas dos estimadores obtidos pelos métodos estudados na Seção 2.2. Desenvolvemos, além disso, estatísticas para testes de hipóteses sobre o vetor de parâmetros de interesse e um estudo de simulação para comparar desempenhos dos testes da razão de verossimilhanças e gradiente para o modelo de regressão beta com erros de medida.

\subsection{Distribuições assintóticas dos estimadores}

\subsubsection{Máxima verossimilhança aproximada}

O método de máxima verossimilhança é um dos mais utilizados em estimação parâmétrica. Os estimadores obtidos a partir deste método apresentam tipicamente boas propriedades como consistência, eficiência e normalidade assintótica (Cramér, 1999, Cap. 28). Para grandes amostras a distribuição aproximada de $\widehat{\Psi}$, estimador de máxima verossimilhança do vetor de parâmetros $\mathbf{\Psi}$, é normal multivariada de média $\boldsymbol{\Psi}$ e matriz de variâncias e covariâncias $I(\widehat{\Psi})^{-1}$ em que $I(\boldsymbol{\Psi})=$ $E\left[-\partial^{2} \ell(\boldsymbol{\Psi}) / \partial \boldsymbol{\Psi} \partial \boldsymbol{\Psi}^{\top}\right]$ é a matriz de informação esperada. O conceito de informação está associado à curvatura média do logaritmo da função de verossimilhança, no sentido de que quanto maior a curvatura mais precisa é a informação contida na verossimilhança, ou equivalentemente, maior o valor de $I(\Psi)$. Podemos considerar $I(\Psi)$ como uma medida de informação global, mas em muitas situações não é possível encontrar os valores esperados, por exemplo, em modelos associados a tempos de vida na presença de censura. É recomendado, nesses casos, o uso de uma medida de informação local, a matriz de informação observada, que é dada por $J(\Psi)=-\partial^{2} \ell(\mathbf{\Psi}) / \partial \mathbf{\Psi} \partial \mathbf{\Psi}^{\top}$. Em modelos não lineares com erros de medida, utilizar a matriz de informação observada no lugar da matriz de informação esperada se torna útil (Buonaccorsi e Tosteson, 1993).

Em muitos casos, o logaritmo da função de verossimilhança depende de integrais difíceis de serem resolvidas. Isto é comum em modelos mistos, modelos com efeitos aleatórios e modelos com erros de medida, como é o caso do modelo tratado nesta tese. Como mencionado anteriormente, existem técnicas numéricas para contornar tal problema de integração. Neste trabalho, usamos o método de quadratura de Gauss-Hermite. A partir do logaritmo da função de verossimilhança aproximada é obtido o vetor escore aproximado, $U_{a}=\partial \ell_{a}(\boldsymbol{\Psi}) / \partial \Psi$, e a matriz de informação observada aproximada, $J_{a}(\boldsymbol{\Psi})=-\partial^{2} \ell_{a}(\boldsymbol{\Psi}) / \partial \mathbf{\Psi} \partial \boldsymbol{\Psi}^{\top}$. Ambas são apresentadas nos Apêndices A.2.1 e A.2.2 para o modelo de regressão beta com erros de medida, respectivamente. A distribuição assintótica do estimador de máxima verossimilhança aproximada é normal com média $\boldsymbol{\Psi}$ e matriz 
de variâncias e covariâncias $J_{a}^{-1}(\Psi)$, isto é, para $n$ e $Q$ suficientemente grande,

$$
n^{1 / 2}(\widehat{\mathbf{\Psi}}-\mathbf{\Psi}) \sim \mathbb{N}\left(\mathbf{0} ; J_{a}^{-1}(\mathbf{\Psi})\right)
$$

aproximadamente (Guolo, 2011). Utilizamos a linguagem de programação Ox para encontrar a matriz de variâncias e covariâncias e erros padrão dos estimadores de máxima verossimilhança aproximada bem como $p$-valores de testes tipo-Wald que usam tais estimadores, tal como mostra o Apêndice B.4.

\subsubsection{Máxima pseudo-verossimilhança aproximada}

Uma técnica usual na estimação dos parâmetros quando o modelo em estudo apresenta parâmetros de interesse e perturbação é a de máxima pseudo-verossimilhança, como vimos na Seção 2.2.2. Nesta seção utilizamos a teoria de equações de estimação para encontrar a distribuição assintótica dos estimadores de máxima pseudo-verossimilhança aproximada tal como é sugerido em Carroll et al. (2006, Seção A.6.6) e Guolo (2011). Carroll e Ruppert (1988) utilizam a teoria de equações de estimação para analisar vários métodos de transformação e ponderação em modelos de regressão; outra boa referência é Godambe (1991).

Na Seção 2.2.2 denotamos os vetores de parâmetros de interesse e perturbação por $\boldsymbol{\theta}$ e $\left(\boldsymbol{\xi}^{\top}, \sigma_{e}^{2}\right)^{\top}$, respectivamente. Como a variância do erro $\sigma_{e}^{2}$ é conhecida ou é estimada à parte quando há réplicas de $w_{t}$, omitiremos o parâmetro $\sigma_{e}^{2}$ na notação. Sejam $\left(y_{t}, \mathbf{w}_{t}\right)$, para $t=1, \ldots, n$, os $n$ pares de variáveis aleatórias observadas, em que a cada t-ésima unidade amostral associa-se uma função de estimação $^{1} \psi_{t}$. O conceito de função de estimação para uma amostra é dada através da seguinte expressão

$$
\Psi_{n}(\mathbf{y}, \mathbf{w} ; \boldsymbol{\theta}, \boldsymbol{\xi})=\sum_{t=1}^{n} \psi_{t}\left(y_{t}, w_{t} ; \boldsymbol{\theta}, \boldsymbol{\xi}\right)
$$

em que $\mathbf{y}=\left(y_{1}, y_{2}, \ldots, y_{n}\right)^{\top}$. Restringimos $(3.1)$ de modo que $\Psi_{n}(y, w ; \widehat{\boldsymbol{\theta}}, \widehat{\boldsymbol{\xi}})=\mathbf{0}$, a qual é denominada de equação de estimação.

Na estimação por máxima pseudo-verossimilhança, conforme apresentado anteriormente, o vetor de parâmetros de perturbação, $\boldsymbol{\xi}$, é estimado a partir de uma equação de estimação,

$$
\sum_{t=1}^{n} \psi_{1 t}\left(w_{t} ; \boldsymbol{\xi}\right)=\mathbf{0}
$$

digamos. Observa-se que a função de estimação $\sum_{t=1}^{n} \psi_{1 t}\left(w_{t} ; \boldsymbol{\xi}\right)$ depende apenas de $w_{t}$ e $\boldsymbol{\xi}$. Após a estimação de $\boldsymbol{\xi}$, estima-se o vetor de parâmetros de interesse a partir de outra equação de estimação da forma

$$
\sum_{t=1}^{n} \psi_{2 t}\left(y_{t}, w_{t} ; \boldsymbol{\theta}, \widehat{\boldsymbol{\xi}}\right)=\mathbf{0}
$$

em que $\widehat{\boldsymbol{\xi}}$ é fixo e obtido em (3.2). Como o par $(\widehat{\boldsymbol{\theta}}, \widehat{\boldsymbol{\xi}})$ é solução simultânea de $(3.2)$ e $(3.3)$, a

\footnotetext{
${ }^{1}$ Seja $\Omega$ um espaço amostral sobre o qual definimos uma familia $\mathfrak{P}$ de distribuições de probabilidade indexadas por um parâmetro $\boldsymbol{\theta}$ desconhecido. Por definição, uma função $\psi: \Omega \times \Theta \longrightarrow \mathbb{R}^{p+r}$ é uma função de estimação se para todo $\boldsymbol{\theta} \in \Theta, \psi(\cdot, \boldsymbol{\theta})$ é uma variável aleatória.
} 
distribuição assintótica de $(\widehat{\boldsymbol{\theta}}, \widehat{\boldsymbol{\xi}})$ é determinada a partir de uma equação de estimação única dada por

$$
\left(\begin{array}{l}
0 \\
0
\end{array}\right)=n^{-1} \sum_{t=1}^{n}\left(\begin{array}{l}
\psi_{1 t}\left(w_{t} ; \boldsymbol{\xi}\right) \\
\psi_{2 t}\left(y_{t}, w_{t} ; \boldsymbol{\theta}, \boldsymbol{\xi}\right)
\end{array}\right)
$$

para mais detalhes, ver Carroll et al. (2006, Seção A.6.6). O objetivo aqui é obter a distribuição assintótica do estimador do vetor de parâmetros de interesse $\boldsymbol{\theta}$. Sob condições de regularidade dadas em Gong e Samaniego (1981), pode-se mostrar (Carroll et al., 2006, Seção A.6.6), que a distribuição assintótica de $\sqrt{n}(\widehat{\boldsymbol{\theta}}-\boldsymbol{\theta})$ é normal com média 0 e matriz de variâncias e covariâncias $\Sigma$ (Carroll et al., 2006; Guolo, 2011), em que

$$
\begin{aligned}
& \Sigma=\mathrm{I}_{\boldsymbol{\theta}}^{-1} \mathrm{I}_{p} \mathrm{I}_{\boldsymbol{\theta}}^{-1},
\end{aligned}
$$

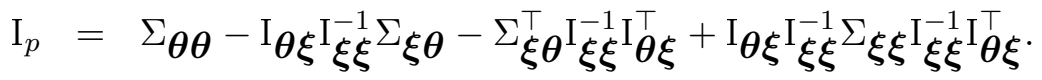

Os componentes da matriz $\Sigma$, como mostra Guolo (2011), são dados por

$$
\begin{aligned}
\mathrm{I}_{\boldsymbol{\theta} \boldsymbol{\theta}} & =-\left.\sum_{t=1}^{n} \mathrm{E}\left\{\frac{\partial^{2} \ell_{p t}(\boldsymbol{\theta}, \boldsymbol{\xi})}{\partial \boldsymbol{\theta} \partial \boldsymbol{\theta}^{\top}}\right\}\right|_{\boldsymbol{\xi}=\widehat{\boldsymbol{\xi}}}, \\
\mathrm{I}_{\boldsymbol{\xi} \boldsymbol{\xi}} & =-\sum_{t=1}^{n} \mathrm{E}\left\{\frac{\partial^{2} \ell_{r t}(\boldsymbol{\xi})}{\left.\partial \boldsymbol{\xi} \partial \boldsymbol{\xi}^{\top}\right\}},\right. \\
\mathrm{I}_{\boldsymbol{\theta} \boldsymbol{\xi}} & =-\left.\sum_{t=1}^{n} \mathrm{E}\left\{\frac{\partial^{2} \ell_{p t}(\boldsymbol{\theta}, \boldsymbol{\xi})}{\partial \boldsymbol{\theta} \partial \boldsymbol{\xi}^{\top}}\right\}\right|_{\boldsymbol{\xi}=\widehat{\boldsymbol{\xi}}}, \\
\Sigma_{\boldsymbol{\theta} \boldsymbol{\theta}} & =\left.\sum_{t=1}^{n} \frac{\partial \ell_{p t}(\boldsymbol{\theta}, \boldsymbol{\xi})}{\partial \boldsymbol{\theta}}\right|_{\boldsymbol{\xi}=\widehat{\boldsymbol{\xi}}}\left\{\left.\frac{\partial \ell_{p t}(\boldsymbol{\theta}, \boldsymbol{\xi})}{\partial \boldsymbol{\theta}}\right|_{\boldsymbol{\xi}=\widehat{\boldsymbol{\xi}}}\right\}^{\top}, \\
\Sigma_{\boldsymbol{\xi} \boldsymbol{\theta}} & =\sum_{t=1}^{n} \frac{\partial \ell_{r t}(\boldsymbol{\xi})}{\partial \boldsymbol{\xi}}\left\{\left.\frac{\partial \ell_{p t}(\boldsymbol{\theta}, \boldsymbol{\xi})}{\partial \boldsymbol{\theta}}\right|_{\boldsymbol{\xi}=\widehat{\boldsymbol{\xi}}}\right\}^{\top}, \\
\Sigma_{\boldsymbol{\xi} \boldsymbol{\xi}} & =\sum_{t=1}^{n} \frac{\partial \ell_{r t}(\boldsymbol{\xi})}{\partial \boldsymbol{\xi}}\left\{\frac{\partial \ell_{r t}(\boldsymbol{\xi})}{\partial \boldsymbol{\xi}}\right\}^{\top},
\end{aligned}
$$

em que $\ell_{r t}(\boldsymbol{\xi})$ e $\ell_{p t}(\boldsymbol{\theta}, \boldsymbol{\xi})$ são as $t$-ésimas parcelas do logaritmo da função de verossimilhança $\ell_{r}(\boldsymbol{\xi})$ e $\ell_{p}(\boldsymbol{\theta}, \boldsymbol{\xi})$ respectivamente, vistos na Seção 2.2.2. Para o caso do modelo de regressão beta com erros de medida, as matrizes $\mathrm{I}_{\boldsymbol{\theta} \boldsymbol{\theta}}, \mathrm{I}_{\boldsymbol{\xi} \boldsymbol{\xi}}$ e I $\boldsymbol{\theta} \boldsymbol{\xi}$ não apresentam formas explícitas. Guolo (2011) sugere utilizar aproximações de Monte Carlo por meio do método de Louis (Louis, 1982; Tanner, 1996, Cap. 4). Neste trabalho, dado que aproximamos numericamente uma integral, sugerimos utilizar simplesmente as segundas derivadas, ou seja, substituir a matriz de informação esperada pela matriz de informação observada (Buonaccorsi e Tosteson, 1993). Deixamos como trabalho futuro investigar a sugestão de Guolo (2011).

As derivadas primeiras e segundas para obter os elementos das matrizes $\mathrm{I}_{\boldsymbol{\theta} \boldsymbol{\theta}}, \mathrm{I}_{\boldsymbol{\xi} \boldsymbol{\xi}}, \mathrm{I}_{\boldsymbol{\theta} \boldsymbol{\xi}}, \Sigma_{\boldsymbol{\theta} \boldsymbol{\theta}}, \Sigma_{\boldsymbol{\xi} \boldsymbol{\theta}}$ e $\Sigma_{\boldsymbol{\xi} \boldsymbol{\xi}}$, do modelo de regressão beta com erros de medida, quando assumimos que uma covariável é medida com erro são dadas nos Apêndices A.2.1, A.2.2 e A.3. No entanto, podemos generalizar facilmente para o caso de $r$ covariáveis medidas com erro. 
Apresentamos no Apêndice B.5 os passos utilizados na linguagem de programação ox para encontrar a matriz de variâncias e covariâncias assintótica, erros padrão e $p$-valores.

\subsubsection{Calibração da regressão}

O método de calibração da regressão visto na Seção 2.2.3 é amplamente utilizado em modelos com erros de medida por sua simplicidade. Recordemos que a ideia central deste método é substituir a variável não observada $x_{t}$ por alguma estimativa da esperança condicional de $x_{t}$ dado $w_{t}$, $E\left(x \mid w_{t}\right)$. O logaritmo da função de verossimilhança modificada (2.19) apresenta vetores de parâmetros de perturbação e interesse, $\left(\boldsymbol{\xi}, \sigma_{e}^{2}\right)^{\top}$ e $\boldsymbol{\theta}$ respectivamente. Assim, concluímos que o logaritmo da função de verossimilhança modificada $\ell_{R C}$ é uma pseudo-verossimilhança da forma como é apresentado em Gong e Samaniego (1981). Diferentemente da abordagem proposta por Carroll et al. (2006, Seção A.6.6) e Guolo (2011), Gong e Samaniego (1981) abordam o método de estimação por máxima pseudo-verossimilhança da seguinte forma. Dada uma amostra de tamanho $n$ com função de distribuição de probabilidade $f(\cdot ; \boldsymbol{\theta}, \boldsymbol{\xi})$, uma estimativa $\widehat{\boldsymbol{\xi}}$ de $\boldsymbol{\xi}$ é obtida por alguma técnica ou alguma outra abordagem distinta da máxima verossimilhança. Então, o estimador de máxima pseudo-verossimilhança, $\widehat{\boldsymbol{\theta}}$, é obtido maximizando o logaritmo da função de verossimilhança $\ell(\cdot ; \boldsymbol{\theta}, \widehat{\boldsymbol{\xi}})$. Espera-se que $\widehat{\boldsymbol{\theta}}$ tenha boas propriedades para grandes amostras quando $\widehat{\boldsymbol{\xi}}$ também tem. Mais detalhes sobre propriedades de consistência e distribuição assintótica de $\boldsymbol{\theta}$ podem ser encontrados em Delgado (1995). Gong e Samaniego (1981) mostram também que, sob condições de regularidade, a distribuição assintótica de $\sqrt{n}(\widehat{\boldsymbol{\theta}}-\boldsymbol{\theta})$ é normal com média 0 e matriz de variâncias e covariâncias $\Sigma^{*}$, em que

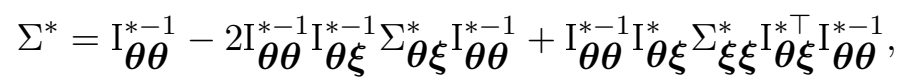

sendo

$$
\begin{aligned}
& \mathrm{I}_{\boldsymbol{\theta} \boldsymbol{\theta}}^{*}=\left.\sum_{t=1}^{n} \mathrm{E}\left\{\left[\frac{\partial \ell_{R C t}(\boldsymbol{\theta}, \boldsymbol{\xi})}{\partial \boldsymbol{\theta}}\right]^{\top}\left[\frac{\partial \ell_{R C t}(\boldsymbol{\theta}, \boldsymbol{\xi})}{\partial \boldsymbol{\theta}}\right]\right\}\right|_{\boldsymbol{\xi}=\widehat{\boldsymbol{\xi}}}, \\
& \mathrm{I}_{\boldsymbol{\theta} \boldsymbol{\xi}}^{*}=\left.\sum_{t=1}^{n} \mathrm{E}\left\{\left[\frac{\partial \ell_{R C t}(\boldsymbol{\theta}, \boldsymbol{\xi})}{\partial \boldsymbol{\theta}}\right]^{\top}\left[\frac{\partial \ell_{R C t}(\boldsymbol{\theta}, \boldsymbol{\xi})}{\partial \boldsymbol{\xi}}\right]\right\}\right|_{\boldsymbol{\xi}=\widehat{\boldsymbol{\xi}}},
\end{aligned}
$$

$\Sigma_{\boldsymbol{\xi} \boldsymbol{\xi}}^{*}$ é a matriz de variâncias e covariâncias do vetor de parâmetros de perturbação e $\ell_{R C t}(\boldsymbol{\theta}, \boldsymbol{\xi})$ é o $t$-ésimo elemento de $\ell_{R C}(\boldsymbol{\theta}, \boldsymbol{\xi})$. Uma análise mais didática e de fácil entendimento sobre a proposta de Gong e Samaniego (1981) é dada por Parke (1986). Parke (1986) mostra que o componente $\Sigma_{\boldsymbol{\theta} \boldsymbol{\xi}}^{*}$ da matriz de variâncias e covariâncias $\Sigma^{*}$ é a matriz nula (Delgado, 1995, Seção 2.2). Considerando o resultado de Parke (1986), a matriz de variâncias e covariâncias $\Sigma^{*}$ dada por Gong e Samaniego (1981) pode ser escrita como

$$
\begin{aligned}
\Sigma^{*} & =\mathrm{I}_{\boldsymbol{\theta} \boldsymbol{\theta}}^{*-1} \mathrm{I}_{R C} \mathrm{I}_{\boldsymbol{\theta} \boldsymbol{\theta}}^{*-1}, \\
\mathrm{I}_{R C} & =\mathrm{I}_{\boldsymbol{\theta} \boldsymbol{\theta}}^{*}+\mathrm{I}_{\boldsymbol{\theta} \boldsymbol{\xi}^{*}}^{\Sigma_{\boldsymbol{\xi}}^{*} \mathrm{I}^{*} \boldsymbol{\theta} \boldsymbol{\xi}}
\end{aligned}
$$

Diferentemente da seção anterior, em que a matriz de informação esperada é aproximada pela matriz de informação observada, aqui para o caso do modelo de regressão beta com erros de medida foi possível calcular os elementos de $\mathbf{I}_{\boldsymbol{\theta} \boldsymbol{\theta}}^{*}$ e $\mathbf{I}_{\boldsymbol{\theta} \boldsymbol{\xi}}^{*}$, pois o logaritmo da função de verossimilhança não 
depende de uma integração. Após alguma álgebra, obtemos

$$
\mathbf{I}_{\boldsymbol{\theta} \boldsymbol{\theta}}^{*}=\left[\begin{array}{rrrr}
\mathbf{Z}^{\top} \Phi \mathbf{U Z} & \mathbf{Z}^{\top} \Phi \mathbf{U x} & \mathbf{Z}^{\top} \mathbf{C G H v} & \mathbf{Z}^{\top} \mathbf{C G H x} \\
& \mathbf{x}^{\top} \Phi \mathbf{U} \mathbf{x} & \mathbf{x}^{\top} \mathbf{C G H v} & \mathbf{x}^{\top} \mathbf{C G H x} \\
& & \mathbf{v}^{\top} \mathbf{D} \mathbf{v} & \mathbf{v}^{\top} \mathbf{D} \mathbf{x} \\
& & & \mathbf{x}^{\top} \mathbf{D} \mathbf{x}
\end{array}\right]_{n(\boldsymbol{\psi}) \times n(\boldsymbol{\psi})},
$$

em que $n(\boldsymbol{\psi})$ representa a dimensão do vetor de parâmetros de interesse, $\mathbf{U}=\operatorname{diag}\left\{u_{1}, \ldots, u_{n}\right\}$, $\Phi=\operatorname{diag}\left\{\phi_{1}, \ldots, \phi_{n}\right\}, \mathbf{H}=\operatorname{diag}\left\{1 / h^{\prime}\left(\phi_{1}\right), \ldots, 1 / h^{\prime}\left(\phi_{n}\right)\right\}, \mathbf{G}=\operatorname{diag}\left\{1 / g^{\prime}\left(\mu_{1}\right), \ldots, 1 / g^{\prime}\left(\mu_{n}\right)\right\}, \mathbf{C}=$ $\operatorname{diag}\left\{c_{1}, \ldots, c_{n}\right\}$ e $\mathbf{D}=\operatorname{diag}\left\{d_{1}, \ldots, d_{n}\right\}, \operatorname{com}$

$$
\begin{aligned}
u_{t} & =\phi_{t}\left\{\psi^{\prime}\left(\mu_{t} \phi_{t}\right)+\psi^{\prime}\left(\left(1-\mu_{t}\right) \phi_{t}\right)\right\} \frac{1}{\left\{g^{\prime}\left(\mu_{t}\right)\right\}^{2}}, \\
c_{t} & =\phi_{t}\left\{\psi^{\prime}\left(\mu_{t} \phi_{t}\right) \mu_{t}-\psi^{\prime}\left[\left(1-\mu_{t}\right) \phi_{t}\right]\left(1-\mu_{t}\right)\right\} \\
d_{t} & =\left[\psi^{\prime}\left(\mu_{t} \phi_{t}\right) \mu_{t}^{2}+\psi^{\prime}\left[\left(1-\mu_{t}\right) \phi_{t}\right]\left(1-\mu_{t}\right)^{2}-\psi^{\prime}\left(\phi_{t}\right)\right] \frac{1}{\left\{h^{\prime}\left(\phi_{t}\right)\right\}^{2}} .
\end{aligned}
$$

De forma análoga, os elementos da matriz I ${ }_{\boldsymbol{\theta}}^{*}$ são dados por

$$
\mathrm{I}_{\boldsymbol{\theta} \boldsymbol{\xi}}^{*}=\left[\begin{array}{rr}
\mathbf{Z}^{\top} \Phi \mathbf{U}^{*} & \mathbf{Z}^{\top} \Phi \mathbf{U}^{\star} \\
\mathbf{x}^{\top} \Phi \mathbf{U}^{*} & \mathbf{x}^{\top} \Phi \mathbf{U}^{\star} \\
\mathbf{v}^{\top} \mathbf{D}^{*} & \mathbf{v}^{\top} \mathbf{D}^{\star} \\
\mathbf{x}^{\top} \mathbf{D}^{*} & \mathbf{x}^{\top} \mathbf{D}^{\star}
\end{array}\right]_{n(\boldsymbol{\psi}) \times 2}
$$

em que $\mathbf{U}^{*}=\operatorname{diag}\left\{u_{1}^{*}, \ldots, u_{n}^{*}\right\}, \mathbf{U}^{\star}=\operatorname{diag}\left\{u_{1}^{\star}, \ldots, u_{n}^{\star}\right\}, \mathbf{D}^{*}=\operatorname{diag}\left\{d_{1}^{*}, \ldots, d_{n}^{*}\right\}, \mathbf{D}^{\star}=\operatorname{diag}\left\{d_{1}^{\star}, \ldots, d_{n}^{\star}\right\}$, $\operatorname{com} u_{t}^{*}=\beta\left(1-k_{x}\right) u_{t}, u_{t}^{\star}=\left(\sigma_{x}^{2}+\sigma_{e}^{2}\right)^{-1} u_{t}^{*}, d_{t}^{*}=\lambda\left(1-k_{x}\right) d_{t}, d_{t}^{\star}=\left(\sigma_{x}^{2}+\sigma_{e}^{2}\right)^{-1} d_{t}^{*}$ e $k_{x}=\sigma_{x}^{2} /\left(\sigma_{x}^{2}+\sigma_{e}^{2}\right)$.

Encontramos a matriz de variâncias e covariâncias dos estimadores, além dos erros padrão e p-valores, tal como mostra o Apêndice B.6.

\subsubsection{Estudo de simulação}

Verificamos o desempenho dos estimadores dos parâmetros do modelo de regressão beta com erros de medida via um estudo de simulação. Consideramos $R=10000$ réplicas de Monte Carlo. Admitimos que o modelo de regressão beta com erros de medida sob o enfoque estrutural apresenta a seguinte estrutura

$$
\begin{aligned}
g\left(\mu_{t}\right) & =\alpha+\beta x_{t}, \\
h\left(\phi_{t}\right) & =\gamma+\lambda x_{t}, \\
w_{t} & =x_{t}+e_{t},
\end{aligned}
$$

em que $t=1,2, \ldots, n$. Selecionamos os seguintes valores para os parâmetros que permanecem fixos para todas as simulações $\alpha=2,0, \beta=-0,6, \gamma=2,5, \lambda=0,5, \mu_{x}=2,5$ e $\sigma_{x}^{2}=2,7$. As ligações logito e logarítmica foram escolhidas, respectivamente, para os parâmetros $\mu_{t}$ e $\phi_{t}$ obtidos a partir das equações (2.4) e (2.5). Geramos a variável dependente $y_{t}$ a partir de uma distribuição beta com média $\mu_{t}$ e precisão $\phi_{t}$. Além disso, geramos a covariável $w_{t}$ a partir de sua distribuição condicional dado $x_{t}$, ou seja, com uma distribuição normal com média $x_{t}$ e variância $\sigma_{e}^{2}$. Consideramos que a variância do erro do modelo $\sigma_{e}^{2}$ é conhecida e atribuímos os seguintes valores a $\sigma_{e}^{2}: 0,05,0,50$ e 1,0 . 
Nas Tabelas 3.1 a 3.4 apresentamos as taxas de cobertura (TC) dos intervalos de confiança com coeficientes de confiança nominal 90\%, 95\% e 99\%. Esta medida foi estimada da seguinte forma

$$
\mathrm{TC}=\frac{\sharp\left\{\Psi_{j} \in \mathrm{IC}\left[\Psi_{j} ; 1-\alpha\right]\right\}}{R},
$$

em que $\Psi_{j}$ é o $j$-ésimo elemento de $\boldsymbol{\Psi}, R$ é o número de réplicas de Monte Carlo e $\operatorname{IC}\left[\Psi_{j} ; 1-\alpha\right]$ é o intervalo de confiança para $\Psi_{j}$ com coeficiente nominal $1-\alpha$.

Tabela 3.1: Taxas de cobertura dos intervalos de confiança. Método de máxima verossimilhança aproximada

\begin{tabular}{c|c|r|r|r|r|r|r|r|r|r}
\cline { 3 - 10 } \multicolumn{2}{c|}{} & \multicolumn{3}{c|}{$n=25$} & \multicolumn{3}{c|}{$n=50$} & \multicolumn{3}{c}{$n=100$} \\
\hline$\sigma_{e}^{2}$ & Parâmetros & $90 \%$ & $95 \%$ & $99 \%$ & $90 \%$ & $95 \%$ & $99 \%$ & $90 \%$ & $95 \%$ & $99 \%$ \\
\hline \multirow{4}{*}{0,05} & $\alpha$ & 83,90 & 89,58 & 92,06 & 88,11 & 93,35 & 95,62 & 88,90 & 94,46 & 96,17 \\
& $\beta$ & 83,44 & 89,01 & 92,17 & 88,24 & 93,51 & 95,87 & 88,96 & 94,18 & 96,68 \\
& $\gamma$ & 87,71 & 92,71 & 96,09 & 89,50 & 94,34 & 97,38 & 89,70 & 94,51 & 97,61 \\
& $\lambda$ & 86,58 & 90,75 & 92,62 & 90,26 & 95,20 & 96,88 & 89,87 & 95,17 & 97,02 \\
\hline \multirow{5}{*}{0,5} & $\alpha$ & 73,42 & 79,86 & 83,94 & 81,05 & 87,24 & 90,80 & 83,77 & 90,19 & 93,08 \\
& $\beta$ & 70,44 & 77,26 & 81,73 & 76,27 & 82,85 & 86,66 & 78,56 & 84,86 & 89,13 \\
& $\gamma$ & 86,17 & 90,66 & 92,63 & 89,41 & 94,61 & 96,83 & 89,12 & 94,72 & 97,34 \\
& $\lambda$ & 63,59 & 71,92 & 82,00 & 68,31 & 75,87 & 84,99 & 70,81 & 78,46 & 87,05 \\
\hline \multirow{5}{*}{1,0} & $\alpha$ & 72,20 & 79,46 & 84,37 & 81,62 & 88,13 & 91,47 & 83,97 & 90,25 & 92,82 \\
& $\beta$ & 69,99 & 77,07 & 81,72 & 77,45 & 84,91 & 88,83 & 80,01 & 86,65 & 90,73 \\
& $\gamma$ & 81,62 & 86,25 & 89,13 & 88,89 & 93,28 & 95,19 & 89,00 & 94,61 & 96,98 \\
& $\lambda$ & 67,10 & 75,52 & 84,37 & 73,71 & 82,39 & 89,58 & 76,63 & 84,73 & 90,79 \\
\hline
\end{tabular}

Tabela 3.2: Taxas de cobertura dos intervalos de confiança. Método de máxima pseudo-verossimilhança aproximada

\begin{tabular}{c|c|r|r|r|r|r|r|r|r|r}
\cline { 3 - 10 } \multicolumn{2}{c|}{} & \multicolumn{3}{c|}{$n=25$} & \multicolumn{3}{c|}{$n=50$} & \multicolumn{3}{|c}{$n=100$} \\
\hline$\sigma_{e}^{2}$ & Parâmetros & $90 \%$ & $95 \%$ & $99 \%$ & $90 \%$ & $95 \%$ & $99 \%$ & $90 \%$ & $95 \%$ & $99 \%$ \\
\hline \multirow{4}{*}{0,05} & $\alpha$ & 81,33 & 87,50 & 90,61 & 84,53 & 90,17 & 92,93 & 84,28 & 90,48 & 93,13 \\
& $\beta$ & 79,36 & 85,06 & 88,55 & 82,16 & 88,16 & 91,34 & 82,47 & 88,28 & 91,67 \\
& $\gamma$ & 77,67 & 83,95 & 89,18 & 81,26 & 87,60 & 92,47 & 83,31 & 89,48 & 93,80 \\
& $\lambda$ & 78,28 & 84,05 & 87,46 & 82,60 & 88,75 & 91,83 & 83,71 & 90,19 & 92,57 \\
\hline \multirow{5}{*}{0,5} & $\alpha$ & 71,80 & 77,75 & 81,09 & 74,43 & 80,54 & 83,88 & 74,09 & 80,40 & 84,16 \\
& $\beta$ & 71,94 & 77,57 & 82,06 & 73,04 & 78,62 & 82,94 & 72,72 & 78,59 & 83,14 \\
& $\gamma$ & 77,02 & 81,93 & 86,29 & 81,77 & 87,56 & 91,36 & 82,91 & 88,75 & 93,24 \\
& $\lambda$ & 62,34 & 69,47 & 75,49 & 62,85 & 70,17 & 76,66 & 65,20 & 72,34 & 77,47 \\
\hline \multirow{5}{*}{1,0} & $\alpha$ & 73,33 & 79,65 & 83,28 & 75,03 & 80,60 & 84,10 & 72,95 & 79,06 & 82,69 \\
& $\beta$ & 74,64 & 80,42 & 84,30 & 73,69 & 79,95 & 83,90 & 71,64 & 77,73 & 82,19 \\
& $\gamma$ & 76,49 & 81,30 & 85,01 & 82,02 & 87,04 & 90,56 & 84,09 & 89,11 & 93,01 \\
& $\lambda$ & 66,42 & 73,62 & 78,68 & 67,44 & 74,47 & 79,98 & 68,16 & 75,38 & 79,46 \\
\hline
\end{tabular}

Observamos nas Tabelas 3.1 e 3.2 que as taxas de cobertura dos intervalos de confiança se aproximam dos coeficientes de confiança nominais à medida em que o tamanho da amostra aumenta, ou seja, a estimação intervalar destes parâmetros apresenta bom desempenho. Já quando utilizamos os métodos de calibração da regressão e naïve (Tabelas 3.3 e 3.4), notamos que as taxas de cobertura foram consideravelmente bem menores do que os coeficientes de confiança nominais considerados aqui, em especial quando a variância do erro aumenta. 
Tabela 3.3: Taxas de cobertura dos intervalos de confiança. Método de calibração da regressão

\begin{tabular}{c|c|r|r|r|r|r|r|r|r|r}
\cline { 3 - 10 } \multicolumn{2}{c|}{} & \multicolumn{3}{c|}{$n=25$} & \multicolumn{3}{c|}{$n=50$} & \multicolumn{3}{c}{$n=100$} \\
\hline$\sigma_{e}^{2}$ & Parâmetros & $90 \%$ & $95 \%$ & $99 \%$ & $90 \%$ & $95 \%$ & $99 \%$ & $90 \%$ & $95 \%$ & $99 \%$ \\
\hline \multirow{4}{*}{0,05} & $\alpha$ & 83,66 & 90,03 & 92,78 & 87,39 & 92,79 & 95,05 & 88,93 & 93,82 & 95,62 \\
& $\beta$ & 82,80 & 89,13 & 92,48 & 86,82 & 92,48 & 95,72 & 88,53 & 93,76 & 96,64 \\
& $\gamma$ & 83,13 & 89,12 & 93,61 & 86,50 & 91,95 & 95,59 & 88,14 & 93,29 & 96,34 \\
& $\lambda$ & 79,59 & 86,17 & 88,86 & 80,61 & 87,84 & 89,10 & 73,85 & 83,05 & 83,45 \\
\hline \multirow{5}{*}{0,5} & $\alpha$ & 81,93 & 88,64 & 91,31 & 85,00 & 91,26 & 93,02 & 85,27 & 91,55 & 92,48 \\
& $\beta$ & 79,70 & 86,56 & 91,27 & 83,51 & 89,72 & 94,53 & 83,01 & 89,75 & 95,55 \\
& $\gamma$ & 79,48 & 86,85 & 89,72 & 79,81 & 87,48 & 89,00 & 74,30 & 82,98 & 83,62 \\
& $\lambda$ & 55,92 & 65,38 & 66,00 & 35,09 & 44,88 & 44,96 & 10,97 & 17,58 & 17,58 \\
\hline \multirow{5}{*}{1,0} & $\alpha$ & 77,62 & 84,78 & 88,22 & 80,26 & 87,58 & 89,94 & 79,56 & 87,24 & 88,27 \\
& $\beta$ & 74,83 & 82,37 & 87,93 & 77,41 & 85,43 & 91,70 & 76,97 & 84,91 & 92,43 \\
& $\gamma$ & 74,64 & 82,79 & 85,01 & 68,87 & 78,59 & 79,46 & 52,63 & 64,42 & 64,64 \\
& $\lambda$ & 50,60 & 59,60 & 60,17 & 27,57 & 36,62 & 36,70 & 6,71 & 10,99 & 10,99 \\
\hline
\end{tabular}

Tabela 3.4: Taxas de cobertura dos intervalos de confiança. Método naïve.

\begin{tabular}{c|c|r|r|r|r|r|r|r|r|r}
\cline { 3 - 10 } \multicolumn{2}{c|}{} & \multicolumn{3}{c|}{$n=25$} & \multicolumn{3}{c|}{$n=50$} & \multicolumn{3}{c}{$n=100$} \\
\hline$\sigma_{e}^{2}$ & Parâmetros & $90 \%$ & $95 \%$ & $99 \%$ & $90 \%$ & $95 \%$ & $99 \%$ & $90 \%$ & $95 \%$ & $99 \%$ \\
\hline \multirow{5}{*}{0,05} & $\alpha$ & 85,37 & 91,08 & 92,88 & 86,96 & 92,46 & 93,62 & 86,23 & 92,20 & 92,96 \\
& $\beta$ & 83,41 & 89,69 & 94,37 & 84,88 & 90,98 & 96,12 & 82,65 & 89,31 & 96,34 \\
& $\gamma$ & 87,02 & 92,63 & 96,47 & 88,05 & 93,19 & 96,99 & 87,98 & 93,54 & 97,25 \\
& $\lambda$ & 84,90 & 91,02 & 92,76 & 82,27 & 89,20 & 90,01 & 73,29 & 82,09 & 82,25 \\
\hline \multirow{5}{*}{0,5} & $\alpha$ & 61,33 & 71,29 & 71,42 & 43,58 & 55,92 & 55,92 & 18,74 & 28,45 & 28,45 \\
& $\beta$ & 47,45 & 58,08 & 75,18 & 23,49 & 33,06 & 55,88 & 4,60 & 8,55 & 21,69 \\
& $\gamma$ & 85,59 & 91,65 & 94,55 & 84,75 & 91,67 & 93,40 & 80,86 & 88,41 & 89,33 \\
& $\lambda$ & 46,96 & 57,60 & 57,82 & 17,73 & 26,58 & 26,58 & 2,05 & 3,49 & 3,49 \\
\hline \multirow{5}{*}{1,0} & $\alpha$ & 35,52 & 46,74 & 46,76 & 13,51 & 20,67 & 20,67 & 1,24 & 2,64 & 2,64 \\
& $\beta$ & 18,49 & 26,81 & 45,66 & 2,94 & 5,39 & 14,63 & 0,04 & 0,12 & 0,60 \\
& $\gamma$ & 81,43 & 88,75 & 90,63 & 74,00 & 83,06 & 83,83 & 57,05 & 68,54 & 68,78 \\
& $\lambda$ & 30,20 & 39,65 & 39,72 & 5,37 & 9,09 & 9,09 & 0,14 & 0,35 & 0,35 \\
\hline
\end{tabular}

\section{$3.2 \quad$ Testes de hipóteses}

Na Seção 2.2 estudamos metodologias para estimar os parâmetros do modelo de regressão beta com erros de medida. Em geral, realizam-se também testes a fim de determinar se hipóteses feitas sobre os parâmetros são suportadas por evidências a partir de dados amostrais. Os procedimentos mais frequentes utilizados na literatura são os testes da razão de verossimilhanças (Wilks, 1938), escore (Rao, 1948) e Wald (Wald, 1943). Uma proposta recente chamada de teste gradiente é dada por Terrell (2002). Uma propriedade em comum destes quatro testes é que são assintoticamente equivalentes sob a hipótese nula e sob alternativas locais (Lemonte e Ferrari, 2012).

A estatística gradiente, foi derivada por Terrell (2002) a partir da estatística escore e da estatística de Wald modificada (Hayakawa e Puri, 1985). A combinação destas duas estatísticas resulta em uma estatística muito simples de ser calculada, não envolvendo nenhum cálculo matricial como produto ou inversa de matrizes. Adicionalmente, ao contrário das estatísticas de Wald e escore, não há necessidade de se obter a matriz de informação esperada ou observada. Lemonte e Ferrari (2012) apresentam uma expansão assintótica para a distribuição da estatística gradiente sob uma 
sequência de alternativas de Pitman convergindo para a hipótese nula a uma taxa de convergência de $n^{-1 / 2}$. Lemonte e Ferrari (2011a,b) mostram que o teste gradiente tem melhor performance que o teste da razão de verossimilhanças para a distribuição Birnbaum-Saunders (BS) sob censura tipo II e em modelo de regressão BS sem censura.

Seja $\boldsymbol{\Psi}=\left(\boldsymbol{\theta}^{\top}, \boldsymbol{\xi}^{\top}, \sigma_{e}^{2}\right)^{\top}$ o vetor de parâmetros do modelo de regressão beta com erros de medida, em que $\boldsymbol{\theta}=\left(\boldsymbol{\alpha}^{\top}, \beta, \boldsymbol{\gamma}^{\top}, \lambda\right)^{\top}$ é o vetor de parâmetros correspondente à média e precisão, $\boldsymbol{\xi}=\left(\mu_{x}, \sigma_{x}^{2}\right)^{\top}$ é o vetor de parâmetros de perturbação e $\sigma_{e}^{2}$ a variância do erro aleatório considerada conhecida ou estimável sempre e quando tenhamos réplicas de $w_{t}$. A seguir estudamos testes de hipóteses sobre o vetor de parâmetros de interesse $\boldsymbol{\theta}$.

Consideremos as seguintes hipóteses:

$$
\begin{aligned}
& \mathcal{H}_{0}: \quad \boldsymbol{\theta}_{(o)}=\boldsymbol{\theta}_{0}, \\
& \mathcal{H}_{1}: \quad \boldsymbol{\theta}_{(o)} \neq \boldsymbol{\theta}_{0},
\end{aligned}
$$

em que $\boldsymbol{\theta}_{(o)}$ é um vetor $o$-dimensional de parâmetros a serem testados e $\boldsymbol{\theta}_{0}$ é um vetor $o$-dimensional cujos elementos são conhecidos. Neste contexto, as estatística a serem estudadas são a razão de verossimilhanças e gradiente. As estatísticas escore e Wald não são consideradas neste estudo, devido ao fato de que se torna inviável o cálculo da matriz de informação de Fisher. As estatísticas da razão de verossimilhanças e gradiente são dadas por

$$
\begin{aligned}
S_{R V} & =2\left\{\ell\left(\widehat{\boldsymbol{\theta}}, \widehat{\boldsymbol{\xi}}, \widehat{\sigma}_{e}^{2}\right)-\ell\left(\widetilde{\boldsymbol{\theta}}_{1}, \widetilde{\boldsymbol{\xi}}, \widehat{\sigma}_{e}^{2}\right)\right\}, \\
S_{G} & =U\left(\widetilde{\boldsymbol{\theta}}_{(o)}\right)^{\top}\left(\widehat{\boldsymbol{\theta}}_{(o)}-\boldsymbol{\theta}_{0}\right),
\end{aligned}
$$

em que $\ell\left(\boldsymbol{\theta}, \boldsymbol{\xi}, \sigma_{e}^{2}\right)$ é o logaritmo da função de verossimilhança, $\left(\widehat{\boldsymbol{\theta}}^{\top}, \widehat{\boldsymbol{\xi}}^{\top}, \widehat{\sigma}_{e}^{2}\right)^{\top}$ e $\left(\widetilde{\boldsymbol{\theta}}^{\top}, \widetilde{\boldsymbol{\xi}}^{\top}, \widehat{\sigma}_{e}^{2}\right)^{\top}$ são, respectivamente, os estimadores de máxima verossimilhança de $\left(\boldsymbol{\theta}^{\top}, \boldsymbol{\xi}^{\top}, \sigma_{e}^{2}\right)^{\top}$ irrestrito e restrito. Recorde que $\widehat{\sigma}_{e}^{2}$ é conhecida ou estimada quando temos réplicas de $w_{t}$. O estimador restrito é obtido pela imposição da hipótese nula. $U\left(\widetilde{\boldsymbol{\theta}}_{(o)}\right)$ é o vetor coluna $o$-dimensional contendo os $o$-elementos da função escore de $\widetilde{\boldsymbol{\theta}}$, til indica que as quantidades são avaliadas no estimador de máxima verossimilhança restrito. Notemos que testar a hipótese nula de homocedasticidade,

$$
\mathcal{H}_{0}: \phi_{1}=\phi_{2}=\ldots=\phi_{n}=\phi
$$

é equivalente a testar $\mathcal{H}_{0}: \gamma_{1}=\gamma_{2}=\ldots=\gamma_{q}=\lambda=0$ no modelo definido em (2.5) com $v_{t 1}=1$, para todo $t=1,2, \ldots, n$.

Para realizar testes de hipóteses em modelos de regressão beta com erros de medida, em que os parâmetros são obtidos via máxima verossimilhança aproximada, pseudo-verossimilhança e calibração da regressão, sugerimos substituir $\ell_{a}, \ell_{p}$ e $\ell_{R C}$ no lugar de $\ell$ em (3.6). Desta forma teremos os testes da razão de verossimilhanças aproximada $\left(S_{R V a}\right)$, razão de pseudo-verossimilhanças $\left(S_{R V p}\right)$ e razão de verossimilhanças de calibração da regressão $\left(S_{R V R C}\right)$, respectivamente. De forma análoga substituímos os vetores escores $U_{a}, U_{p}$ e $U_{R C}$ no lugar de $U$ em (3.7), e assim teremos as estatísticas gradiente aproximada $S_{G a}$, pseudo-gradiente $S_{G p}$ e gradiente de calibração da regressão $S_{G R C}$ respectivamente.

Sob condições de regularidade e sob $\mathcal{H}_{0}$, as estatísticas $S_{R V a}$ e $S_{G a}$ convergem em distribuição para $\chi_{o}^{2}$. Desse modo, os testes podem ser realizados usando valores críticos aproximados obti- 
dos como quantis da distribuição qui-quadrado com $o$ graus de liberdade. No entanto, segundo Liang e Self (1996) e Geys et al. (1999) as estatísticas $S_{R V p}$ e $S_{R V R C}$ convergem em distribuição para

$$
\chi^{*}=\sum_{j=1}^{P} \tau_{j} \chi_{j},
$$

em que $P=(p+1)+(q+1), \chi_{j^{\prime} s}$ são independentes com distribuição $\chi_{1}^{2}$ e $\tau_{1} \geq \ldots \geq \tau_{P}$ são autovalores de $\mathrm{I}_{\boldsymbol{\theta} \boldsymbol{\theta}}^{-1} \mathrm{I}_{p}$ ou $\mathrm{I}_{\boldsymbol{\theta} \boldsymbol{\theta}}^{-1} \mathrm{I}_{R C}$ conforme o caso, em que $\mathrm{I}_{p}$ e $\mathrm{I}_{R C}$ são dadas em (3.4) e (3.5). Consideramos $\mathrm{I}_{\boldsymbol{\theta} \boldsymbol{\theta}}^{-1} \mathrm{I}_{p}$ se estimamos os parâmetros via máxima pseudo-verossimilhança e $\mathrm{I}_{\boldsymbol{\theta} \boldsymbol{\theta}}^{-1} \mathrm{I}_{R C}$ se estimamos via calibração da regressão. Analogamente, é fácil mostrar que as estatísticas $S_{G p}$ e $S_{G R C}$ convergem em distribuição para (3.8). Geys et al. (1999) sugerem considerar $S_{R V p}^{a}=S_{R V p} / \bar{\tau}$ que representa a estatística de teste da razão pseudo-verossimilhanças ajustada, em que $\bar{\tau}$ é a média aritmética dos autovalores $\tau_{j}$, para $j=1, \ldots, P$. Desse modo, os autores mostram que, $S_{R V p}^{a}$ converge em distribuição para $\chi_{P}^{2}$. De forma análoga podemos encontrar $S_{R V R C}^{a}, S_{G p}^{a}$ e $S_{G R C}^{a}$.

Estudos de simulação são muito importantes na validação teórica; a seguir apresentamos um estudo que objetiva avaliar o desempenho dos testes descritos acima.

\subsubsection{Estudo de simulação}

Estudos de simulação são conduzidos aqui com o fim de avaliar os tamanhos dos testes, comparando os desempenhos dos testes da razão de verossimilhanças e gradiente. Os desempenhos são avaliados em função da proximidade das probabilidades de rejeição da hipótese nula, sendo esta verdadeira, aos respectivos níveis nominais dos testes. Consideramos o modelo de regressão beta com erros de medida em que $g\left(\mu_{t}\right)=\alpha+\beta x_{t}, h\left(\phi_{t}\right)=\gamma+\lambda x_{t}, w_{t}=x_{t}+e_{t}$, sendo $x_{t} \sim N\left(\mu_{x}, \sigma_{x}^{2}\right)$ e $e_{t} \sim N\left(0, \sigma_{e}^{2}\right)$. Realizamos testes das seguintes hipóteses sobre o parâmetro de interesse $\boldsymbol{\theta}=(\alpha, \beta, \gamma, \lambda)^{\top}$, sob os seguintes cenários:

Cenário $1 \mathcal{H}_{0}: \beta=0(o=1)$,

Cenário $2 \mathcal{H}_{0}: \lambda=0(o=1)$,

contra as alternativas bilaterais. Assumimos que os valores verdadeiros dos parâmetros são $\alpha=2,0$, $\beta=0,0, \gamma=2,5, \lambda=0,6, \mu_{x}=2,5$ e $\sigma_{e}^{2}=2,7$, para o cenário 1 , e $\alpha=2,0, \beta=-0,6, \gamma=2,5, \lambda=0,0$, $\mu_{x}=2,5$ e $\sigma_{x}^{2}=2,7$, para o cenário 2 . Consideramos que a variância do erro de medição em $x_{t}, \sigma_{e}^{2}$, é conhecida a qual atribuímos os seguintes valores: 0,05, 0,50 e 1,0. Amostras de tamanhos $n=25,50$ e 100 foram consideradas. Para avaliar numericamente os testes, foram realizadas 10000 réplicas de Monte Carlo, considerando três níveis nominais 1\%, 5\% e 10\%. Geramos amostras de Monte Carlo como na Seção 2.3. Para cada tamanho amostral e cada nível nominal considerado, calculamos as taxas de rejeição de cada teste (razão da verossimilhanças e gradiente), isto é, estimamos, via simulação, $P\left(S_{R V}>x_{\alpha}\right), P\left(S_{G}>x_{\alpha}\right)$, em que $S_{R V}$ e $S_{G}$ foram dadas em (3.6) e (3.7) e $x_{\alpha}$ representa o quantil de ordem $1-\alpha$ da distribuição $\chi_{1}^{2}$ quando são estimados os parâmetros via máxima verossimilhança aproximada e naïve. No entanto, quando estimamos os parâmetros via pseudo-máxima verossimilhança e calibração da regressão $x_{\alpha}$ representa o quantil de ordem $1-\alpha$ da distribuição $\chi_{P}^{2}$.

As Tabelas 3.5 a 3.8 mostram os resultados obtidos no cenário 1 para os diferentes valores de $\sigma_{e}^{2}$. Notamos uma redução na distorção do tamanho do teste à medida em que o número de observações 
cresce, quando utilizamos os métodos de máxima verossimilhança aproximada e máxima pseudoverossimilhança aproximada independente da variância do erro. Os testes que usam estimadores obtidos via calibração da regressão e naïve também têm bom desempenho mas são ligeiramente mais afetados pela presença de erros de medida.

Tabela 3.5: Taxa de rejeição sob $\mathcal{H}_{0}: \beta=0$ dos testes da razão de verossimilhanças e gradiente. Método de máxima verossimilhança aproximada

\begin{tabular}{c|c|r|r|r|r|r|r|r|r|r}
\cline { 3 - 11 } \multicolumn{2}{c|}{} & \multicolumn{3}{c|}{$n=25$} & \multicolumn{3}{c|}{$n=50$} & \multicolumn{3}{c}{$n=100$} \\
\hline$\sigma_{e}^{2}$ & Testes & $1 \%$ & $5 \%$ & $10 \%$ & $1 \%$ & $5 \%$ & $10 \%$ & $1 \%$ & $5 \%$ & $10 \%$ \\
\hline \multirow{2}{*}{0,05} & RV & 1,81 & 7,54 & 13,43 & 1,32 & 6,29 & 11,61 & 1,12 & 5,13 & 10,54 \\
\cline { 2 - 11 } & Gradiente & 0,85 & 5,72 & 11,79 & 0,74 & 5,41 & 10,85 & 0,93 & 4,69 & 10,11 \\
\hline \multirow{2}{*}{0,5} & RV & 1,78 & 7,65 & 13,92 & 1,34 & 6,21 & 11,61 & 1,17 & 5,57 & 10,77 \\
\cline { 2 - 10 } & Gradiente & 0,64 & 5,28 & 11,01 & 0,73 & 4,84 & 9,99 & 0,82 & 4,84 & 9,84 \\
\hline \multirow{2}{*}{1,0} & RV & 1,70 & 7,48 & 13,91 & 1,19 & 5,63 & 11,35 & 1,08 & 5,66 & 11,36 \\
\cline { 2 - 10 } & Gradiente & 0,87 & 4,71 & 10,11 & 0,68 & 4,05 & 9,30 & 0,62 & 4,51 & 10,01 \\
\hline
\end{tabular}

Tabela 3.6: Taxa de rejeição sob $\mathcal{H}_{0}: \beta=0$ dos testes da razão de verossimilhanças e gradiente. Método de máxima pseudo-verossimilhança aproximada

\begin{tabular}{c|c|r|r|r|r|r|r|r|r|r}
\cline { 3 - 11 } \multicolumn{2}{c|}{} & \multicolumn{3}{c|}{$n=25$} & \multicolumn{3}{|c|}{$n=50$} & \multicolumn{3}{|c}{$n=100$} \\
\hline$\sigma_{e}^{2}$ & Testes & $1 \%$ & $5 \%$ & $10 \%$ & $1 \%$ & $5 \%$ & $10 \%$ & $1 \%$ & $5 \%$ & $10 \%$ \\
\hline \multirow{2}{*}{0,05} & RV & 1,92 & 6,98 & 13,36 & 1,24 & 6,22 & 11,99 & 1,13 & 5,32 & 10,52 \\
\cline { 2 - 11 } & Gradiente & 0,88 & 5,46 & 11,58 & 0,75 & 5,32 & 11,15 & 0,85 & 4,83 & 10,16 \\
\hline \multirow{2}{*}{0,5} & RV & 1,67 & 7,53 & 14,02 & 1,37 & 6,61 & 12,16 & 1,14 & 5,44 & 10,69 \\
\cline { 2 - 11 } & Gradiente & 0,78 & 5,05 & 11,09 & 0,71 & 5,31 & 10,75 & 0,88 & 4,83 & 9,90 \\
\hline \multirow{2}{*}{1,0} & RV & 2,32 & 7,98 & 14,34 & 1,42 & 6,44 & 12,28 & 1,19 & 5,61 & 10,84 \\
\cline { 2 - 10 } & Gradiente & 0,89 & 4,98 & 10,72 & 0,86 & 4,78 & 10,50 & 0,83 & 4,69 & 9,89 \\
\hline
\end{tabular}

Tabela 3.7: Taxa de rejeição sob $\mathcal{H}_{0}: \beta=0$ dos testes da razão de verossimilhanças e gradiente. Método de calibração da regressão

\begin{tabular}{c|c|r|r|r|r|r|r|r|r|r}
\cline { 3 - 10 } \multicolumn{2}{c|}{} & \multicolumn{3}{c|}{$n=25$} & \multicolumn{3}{|c|}{$n=50$} & \multicolumn{3}{c}{$n=100$} \\
\hline$\sigma_{e}^{2}$ & Testes & $1 \%$ & $5 \%$ & $10 \%$ & $1 \%$ & $5 \%$ & $10 \%$ & $1 \%$ & $5 \%$ & $10 \%$ \\
\hline \multirow{2}{*}{0,05} & RV & 1,82 & 7,27 & 13,20 & 1,30 & 5,93 & 11,47 & 1,17 & 5,59 & 10,65 \\
\cline { 2 - 11 } & Gradiente & 0,84 & 5,67 & 11,57 & 0,84 & 5,24 & 10,73 & 1,02 & 5,25 & 10,30 \\
\hline \multirow{2}{*}{0,5} & RV & 1,82 & 6,93 & 13,12 & 1,63 & 6,31 & 11,34 & 0,94 & 5,28 & 10,63 \\
\cline { 2 - 10 } & Gradiente & 0,77 & 5,61 & 11,41 & 1,09 & 5,51 & 10,63 & 0,82 & 4,89 & 10,17 \\
\hline \multirow{2}{*}{1,0} & RV & 1,93 & 7,78 & 13,73 & 1,55 & 5,81 & 11,30 & 1,17 & 5,68 & 11,02 \\
\cline { 2 - 10 } & Gradiente & 0,99 & 6,10 & 11,96 & 1,13 & 5,14 & 10,40 & 0,92 & 5,24 & 10,52 \\
\hline
\end{tabular}

Com relação às Tabelas 3.9 a 3.12, que representam os resultados de simulação do cenário 2, observamos que os tamanhos empíricos dos testes aproximam-se dos níveis de significância nominais com o aumento do tamanho da amostra, quando utilizamos os métodos de estimação por máxima verossimilhança aproximada e pseudo-verossimilhança aproximada. Novamente, os testes que usam estimadores de calibração da regressão e naïve são um pouco mais afetados pela presença de erros de medição. Na maioria dos casos os testes são considerados liberais, ou seja, seus tamanhos empíricos estão sempre acima do nível nominal adotado. 
Tabela 3.8: Taxa de rejeição sob $\mathcal{H}_{0}: \beta=0$ dos testes da razão de verossimilhanças e gradiente. Método naïve

\begin{tabular}{c|c|r|r|r|r|r|r|r|r|r}
\cline { 3 - 10 } \multicolumn{2}{c|}{} & \multicolumn{3}{c|}{$n=25$} & \multicolumn{3}{c|}{$n=50$} & \multicolumn{3}{c}{$n=100$} \\
\hline$\sigma_{e}^{2}$ & Testes & $1 \%$ & $5 \%$ & $10 \%$ & $1 \%$ & $5 \%$ & $10 \%$ & $1 \%$ & $5 \%$ & $10 \%$ \\
\hline \multirow{2}{*}{0,05} & RV & 1,82 & 7,30 & 13,22 & 1,18 & 5,92 & 11,26 & 1,17 & 5,57 & 11,01 \\
\cline { 2 - 11 } & Gradiente & 0,84 & 5,69 & 11,60 & 0,81 & 5,19 & 10,60 & 1,04 & 5,21 & 10,55 \\
\hline \multirow{2}{*}{0,5} & RV & 1,82 & 7,27 & 13,17 & 1,64 & 6,26 & 11,33 & 1,05 & 5,49 & 10,76 \\
\cline { 2 - 10 } & Gradiente & 0,85 & 5,84 & 11,56 & 1,10 & 5,46 & 10,62 & 0,89 & 5,10 & 10,43 \\
\hline \multirow{2}{*}{1,0} & RV & 1,97 & 7,79 & 14,05 & 1,45 & 6,15 & 11,41 & 1,08 & 5,52 & 10,74 \\
\cline { 2 - 10 } & Gradiente & 0,97 & 6,08 & 12,53 & 0,88 & 5,44 & 10,58 & 0,86 & 5,14 & 10,34 \\
\hline
\end{tabular}

Tabela 3.9: Taxa de rejeição sob $\mathcal{H}_{0}: \lambda=0$ dos testes da razão de verossimilhança e gradiente, no modelo de regressão beta com erros de medida. Método de máxima verossimilhança aproximada

\begin{tabular}{c|c|r|r|r|r|r|r|r|r|r}
\cline { 3 - 11 } \multicolumn{2}{c|}{} & \multicolumn{3}{c|}{$n=25$} & \multicolumn{3}{|c|}{$n=50$} & \multicolumn{3}{|c}{$n=100$} \\
\hline$\sigma_{e}^{2}$ & Testes & $1 \%$ & $5 \%$ & $10 \%$ & $1 \%$ & $5 \%$ & $10 \%$ & $1 \%$ & $5 \%$ & $10 \%$ \\
\hline \multirow{2}{*}{0,05} & RV & 1,54 & 6,42 & 12,07 & 1,20 & 5,64 & 11,31 & 0,99 & 4,73 & 9,67 \\
\cline { 2 - 11 } & Gradiente & 1,43 & 6,11 & 11,85 & 1,19 & 5,53 & 11,25 & 0,96 & 4,69 & 9,62 \\
\hline \multirow{2}{*}{0,5} & RV & 3,19 & 9,11 & 14,98 & 1,30 & 5,62 & 11,03 & 0,94 & 5,03 & 10,64 \\
\cline { 2 - 11 } & Gradiente & 2,87 & 8,24 & 14,02 & 1,56 & 5,83 & 11,14 & 1,02 & 5,18 & 10,74 \\
\hline \multirow{2}{*}{1,0} & RV & 5,96 & 14,22 & 20,87 & 2,25 & 7,82 & 13,33 & 1,26 & 5,89 & 11,37 \\
\cline { 2 - 10 } & Gradiente & 3,76 & 9,94 & 15,60 & 2,29 & 7,53 & 12,57 & 1,42 & 6,04 & 11,41 \\
\hline
\end{tabular}

Nas condições do estudo de simulação apresentado nesta seção, podemos concluir que testes de hipóteses no modelo de regressão beta com erros de medida são pouco afetados pela presença de erros de medição. Esse resultado é de muito interessante nos casos práticos, dado que podemos inicialmente realizar testes de hipóteses para selecionar um modelo adequado utilizando simplesmente o método naïve. 
Tabela 3.10: Taxa de rejeição sob $\mathcal{H}_{0}: \lambda=0$ dos testes da razão de verossimilhança e gradiente, no modelo de regressão beta com erros de medida. Método de máxima pseudo-verossimilhança aproximada

\begin{tabular}{c|c|r|r|r|r|r|r|r|r|r}
\cline { 2 - 10 } \multicolumn{2}{c|}{} & \multicolumn{3}{c|}{$n=25$} & \multicolumn{3}{|c|}{$n=50$} & \multicolumn{3}{|c}{$n=100$} \\
\hline$\sigma_{e}^{2}$ & Testes & $1 \%$ & $5 \%$ & $10 \%$ & $1 \%$ & $5 \%$ & $10 \%$ & $1 \%$ & $5 \%$ & $10 \%$ \\
\hline \multirow{2}{*}{0,05} & RV & 1,35 & 6,36 & 12,21 & 1,26 & 5,54 & 10,79 & 0,93 & 5,03 & 10,17 \\
\cline { 2 - 11 } & Gradiente & 1,28 & 6,18 & 11,87 & 1,21 & 5,50 & 10,66 & 0,92 & 4,97 & 10,17 \\
\hline \multirow{2}{*}{0,5} & RV & 3,19 & 9,16 & 15,27 & 1,14 & 5,30 & 10,83 & 0,85 & 4,59 & 9,33 \\
\cline { 2 - 10 } & Gradiente & 2,93 & 8,33 & 14,46 & 1,33 & 5,46 & 10,98 & 0,89 & 4,72 & 9,58 \\
\hline \multirow{2}{*}{1,0} & RV & 4,38 & 12,51 & 19,15 & 1,87 & 6,81 & 12,47 & 0,96 & 4,88 & 9,72 \\
\cline { 2 - 10 } & Gradiente & 2,94 & 8,70 & 14,62 & 1,77 & 6,57 & 12,06 & 1,17 & 5,08 & 10,16 \\
\hline
\end{tabular}

Tabela 3.11: Taxa de rejeição sob $\mathcal{H}_{0}: \lambda=0$ dos testes da razão de verossimilhança e gradiente, no modelo de regressão beta com erros de medida. Método de calibração da regressão

\begin{tabular}{c|c|r|r|r|r|r|r|r|r|r}
\cline { 3 - 11 } \multicolumn{2}{c|}{} & \multicolumn{3}{c|}{$n=25$} & \multicolumn{3}{|c|}{$n=50$} & \multicolumn{3}{|c}{$n=100$} \\
\hline$\sigma_{e}^{2}$ & Testes & $1 \%$ & $5 \%$ & $10 \%$ & $1 \%$ & $5 \%$ & $10 \%$ & $1 \%$ & $5 \%$ & $10 \%$ \\
\hline \multirow{2}{*}{0,05} & RV & 1,43 & 6,51 & 12,16 & 1,27 & 5,71 & 10,93 & 0,95 & 5,03 & 10,19 \\
\cline { 2 - 11 } & Gradiente & 1,27 & 6,23 & 11,88 & 1,21 & 5,63 & 10,79 & 0,96 & 5,01 & 10,17 \\
\hline \multirow{2}{*}{0,5} & RV & 1,73 & 7,67 & 13,70 & 1,22 & 5,84 & 11,21 & 1,03 & 5,52 & 11,08 \\
\cline { 2 - 10 } & Gradiente & 1,53 & 7,42 & 13,29 & 1,15 & 5,72 & 11,04 & 1,01 & 5,53 & 11,08 \\
\hline \multirow{2}{*}{1,0} & RV & 1,89 & 7,27 & 13,29 & 1,48 & 6,34 & 11,90 & 1,56 & 6,33 & 12,18 \\
\cline { 2 - 10 } & Gradiente & 1,58 & 7,04 & 12,87 & 1,44 & 6,15 & 11,85 & 1,47 & 6,31 & 12,13 \\
\hline
\end{tabular}

Tabela 3.12: Taxa de rejeição sob $\mathcal{H}_{0}: \lambda=0$ dos testes da razão de verossimilhança e gradiente, no modelo de regressão beta com erros de medida. Método naïve

\begin{tabular}{c|c|r|r|r|r|r|r|r|r|r}
\cline { 3 - 10 } \multicolumn{2}{c|}{} & \multicolumn{3}{c|}{$n=25$} & \multicolumn{3}{|c|}{$n=50$} & \multicolumn{3}{c}{$n=100$} \\
\hline$\sigma_{e}^{2}$ & Testes & $1 \%$ & $5 \%$ & $10 \%$ & $1 \%$ & $5 \%$ & $10 \%$ & $1 \%$ & $5 \%$ & $10 \%$ \\
\hline \multirow{2}{*}{0,05} & RV & 1,50 & 6,47 & 12,31 & 1,08 & 5,49 & 10,99 & 1,02 & 5,08 & 9,72 \\
\cline { 2 - 11 } & Gradiente & 1,24 & 6,10 & 11,97 & 1,02 & 5,38 & 10,83 & 1,02 & 5,08 & 9,73 \\
\hline \multirow{2}{*}{0,5} & RV & 1,67 & 6,51 & 12,65 & 1,34 & 5,94 & 11,51 & 1,22 & 5,93 & 11,38 \\
\cline { 2 - 10 } & Gradiente & 1,38 & 6,23 & 12,36 & 1,26 & 5,89 & 11,40 & 1,18 & 5,88 & 11,32 \\
\hline \multirow{2}{*}{1,0} & RV & 1,78 & 6,97 & 12,68 & 1,61 & 6,50 & 11,99 & 1,72 & 6,84 & 12,67 \\
\cline { 2 - 10 } & Gradiente & 1,67 & 6,69 & 12,20 & 1,50 & 6,38 & 11,78 & 1,75 & 6,78 & 12,65 \\
\hline
\end{tabular}




\section{Capítulo 4}

\section{Análise de diagnóstico}

\subsection{Introdução}

A análise de diagnóstico é uma etapa importante na análise de um modelo ajustado para verificar a existência de possíveis afastamentos das suposições do modelo. A metodologia de diagnóstico iniciou-se com a análise de resíduos para detectar a presença de pontos extremos e avaliar a adequação da distribuição proposta para a variável resposta. A análise de resíduos num modelo estatístico pode ser baseada nos resíduos ordinários, em versões padronizadas, em resíduos construídos a partir dos componentes da função desvio (McCullagh e Nelder, 1999, Cap.2) ou em resíduos generalizados (Cox e Snell, 1968).

Muitos trabalhos têm sido publicados acerca de análise de resíduos em modelos de regressão, destacando-se o trabalho de Pregibon (1981), que propõe o componente do desvio como resíduo na classe dos modelos lineares generalizados; McCullagh (1987, Cap. 6) apresenta uma padronização do componente do desvio em que se procura corrigir os efeitos de assimetria e curtose; Atkinson (1985) propõe a construção por simulação de Monte Carlo de uma banda de confiança para os resíduos da regressão normal linear, a qual denominou envelope, e que permite melhor avaliar se os resíduos têm a distribuição esperada sob as suposições do modelo; Williams (1987) discute a construção de envelopes em modelo lineares generalizados; Ferrari e Cribari-Neto (2004), Espinheira et al. (2008a) e Espinheira et al. (2008b) apresentam resíduos padronizados para modelos de regressão beta; Fuller (1987, Seção 2.2.2), Carroll e Spiegelman (1992) e Buonaccorsi (2010, Seção 4.7) apresentam resíduos na presença de erros de medição, sugerindo a construção de gráficos dos resíduos versus as estimativas dos valores preditos da variável não observada.

A estratégia de eliminar pontos é uma técnica usual para avaliar o impacto da retirada de uma observação particular nas estimativas dos parâmetros. A distância de Cook (1977), originalmente desenvolvida para modelos normais lineares, foi rapidamente assimilada e estendida para diversas classes de modelos. A eliminação individual de pontos pode ocasionar um problema conhecido como efeito de mascaramento, ou seja, deixar de detectar pontos conjuntamente discrepantes.

Outro aspecto importante na análise de diagnóstico é a detecção de observações influentes. Cook (1986) propõe avaliar a influência de pontos por meio do estudo da curvatura de seç̧ões normais. Embora o método de Cook (1986) seja de grande utilidade, ele apresenta alguns inconvenientes. Por exemplo, a curvatura normal pode assumir valores nos reais positivos e não é invariante sob reparametrizações de escala. Com o objetivo de contornar estas dificuldades Poon e Poon (1999) propõem uma nova medida da curvatura, sendo esta de fácil interpretação e invariante sob reparametrizações de escala. Zhu e Zhang (2004) apresentam, por sua vez, testes de especificidade do modelo com base na curvatura de secções normais como também a equivalência assintótica de algumas medidas de 
eliminação de pontos. Medidas de influência local conforme (Poon e Poon, 1999) foram estudadas por Ospina (2008) em modelo de regressão beta inflacionado.

Este capítulo encontra-se organizado da seguinte forma. Na Seção 4.2 adaptamos os resíduos ponderados padronizados propostos por Espinheira et al. (2008a,b) para os modelos de regressão beta na presença de erros de medição na covariável. Na Seção 4.3 desenvolvemos a análise de influência global, definindo medidas tais como a distância de Cook generalizada e o afastamento da verossimilhança. Na Seção 4.4 desenvolvemos a análise de influência local baseada nas propostas de Cook (1986) e Poon e Poon (1999). Consideramos três esquemas de perturbação, a saber: a ponderação de casos, perturbação individual da variável resposta e a perturbação individual de covariáveis com ou livre de erros de medição.

\subsection{Análise de Resíduos}

Quando se adota um modelo de regressão e este é ajustado a um conjunto de dados, a avaliação desse ajuste pode ser realizada por meio da análise dos resíduos. A partir dessa análise identificam-se observações discrepantes, além de avaliar a existência de afastamentos sérios das suposições inerentes ao modelo. Se o modelo for adequado, gráficos de resíduos versus ordens das observações ou valores preditos devem apresentar um comportamento aleatório em torno de zero. Define-se como resíduo uma medida cujo objetivo é identificar discrepância entre o modelo ajustado e os dados. Assim, a maioria das formulações dos resíduos baseia-se na diferença $y_{t}-\widehat{E\left(y_{t}\right)}$. No entanto, respeitando o formato da distribuição de probabilidade da variável resposta, é mais interessante pensar no resíduo como uma função $r\left(y_{t}, \widehat{\mathrm{E}\left(y_{t}\right)}\right)$, definição geral de resíduos proposta por Cox e Snell (1968). Sob este ponto de vista, Ferrari e Cribari-Neto (2004), Espinheira et al. (2008a)e Espinheira et al. (2008b) propõem resíduos para o modelo de regressão beta. Com base nessas referências, adaptamos o resíduo ponderado padronizado proposto por Espinheira et al. (2008a) ao modelo de regressão beta na presença de erros de medição.

\subsubsection{Resíduos ponderados padronizados}

Para a classe de modelos de regressão beta com dispersão constante, Ferrari e Cribari-Neto (2004) definem inicialmente o resíduo ordinário, dado por

$$
r_{t}=\frac{y_{t}-\widehat{\mu}_{t}}{\sqrt{\widehat{\operatorname{Var}}\left(y_{t}\right)}},
$$

em que $\widehat{\mu}_{t}=g^{-1}(\cdot)$ e $\widehat{\operatorname{Var}}\left(y_{t}\right)=\widehat{\mu}_{t}\left(1-\widehat{\mu_{t}}\right) /(1+\widehat{\phi})$. Posteriormente, Espinheira et al. (2008a) sugerem utilizar resíduos padronizados obtidos da convergência do processo iterativo escore de Fisher para estimação dos parâmetros de regressão. Sob este ponto de vista, propõem os chamados resíduos ponderados padronizados 1 e 2 e constatam, via estudo de simulação, que o resíduo ponderado padronizado 2 apresenta desempenho superior, especialmente no sentido de identificar observações influentes. O resíduo ponderado padronizado 2 para o modelo de regressão beta com dispersão variável, segundo Espinheira et al. (2008a), é dado por

$$
r_{t}^{p p}=\frac{y_{t}^{*}-\widehat{\mu}_{t}^{*}}{\sqrt{v_{t}\left(1-h_{t t}^{*}\right)}}
$$


para $t=1, \ldots, n$, sendo $\mu_{t}^{*}=\psi\left(\mu_{t} \phi_{t}\right)-\psi\left[\left(1-\mu_{t}\right) \phi_{t}\right], v_{t}=\psi^{\prime}\left(\mu_{t} \phi_{t}\right)+\psi^{\prime}\left[\left(1-\mu_{t}\right) \phi_{t}\right]$ e $h_{t t}^{*}$ é o $t$-ésimo elemento da diagonal principal de

$$
\mathbf{H}^{*}=(\widehat{\mathbf{M}} \boldsymbol{\Phi})^{1 / 2} \mathbf{W}\left(\mathbf{W}^{\top} \mathbf{\Phi} \widehat{\mathbf{M}} \mathbf{W}\right)^{-1} \mathbf{W}^{\top}(\mathbf{\Phi} \widehat{\mathbf{M}})^{1 / 2},
$$

em que $\mathbf{M}=\operatorname{diag}\left\{m_{1}, \ldots, m_{n}\right\} \operatorname{com} m_{t}=\phi_{t} v_{t} /\left[g^{\prime}\left(\mu_{t}\right)\right]^{2}, \mathbf{\Phi}=\operatorname{diag}\left\{\phi_{1}, \ldots, \phi_{n}\right\}$ e $\mathbf{W}$ é a matriz de variáveis preditoras $n \times[(p+1)+(\breve{q}+1)]$.

Quando os modelos de regressão apresenta erros de medição, é sugerido por Fuller (1987, Seção 2.2.2), Carroll e Spiegelman (1992) e Buonaccorsi (2010, Seção 4.7) avaliar o $t$-ésimo resíduo baseado na estimativa corrigida (estimativa obtida quando consideramos a presença de erros de medição na variável) e nos valores observados. Desta forma, propomos o uso dos resíduos padronizados padronizados 2, expressão (4.1), com as estimativas obtidas pelos métodos de máxima verossimilhança aproximada, máxima pseudo-verossimilhança aproximada e calibração da regressão.

Gráficos dos resíduos, $r_{t}^{p p}$, versus índice das observações não são sempre adequados para verificar a existência de possíveis afastamentos das suposições intrínsecas ao modelo quando há presença de erros de medição; ver, por exemplo, Fuller (1987, Seção 2.2.2), Carroll e Spiegelman (1992) e Buonaccorsi (2010, Seção 4.7). Sob modelos com erros de medição é recomendado realizar gráficos dos resíduos, versus valores preditos, $\widehat{x}_{t}$, da variável não observada, $x_{t}$. Em modelos não lineares na presença de erros de medição, é extremamente complicada a obtenção de $\widehat{x}_{t}$, de modo que Fuller (1987, Seção 2.2.2) sugere utilizar uma estimativa consistente do valor esperado de $x_{t}$ dado $w_{t}$, $\mathrm{E} \widehat{\left(x_{t} \mid w_{t}\right)}$, no lugar de $\widehat{x}_{t}$.

Os passos para encontrar os resíduos ponderados padronizados quando a covariável é medida com erro na linguagem de programação Ox são dados no Apêndice B.7.

Com o intuito de investigar a distribuição empírica dos resíduos ponderados padronizados 2 na presença de erros de medição, realizamos um estudo de simulação de Monte Carlo com 10000 réplicas. Consideramos o modelo de regressão beta com

$$
\begin{array}{r}
g\left(\mu_{t}\right)=\alpha+\beta x_{t}, \\
h\left(\phi_{t}\right)=\gamma+\lambda x_{t}, \\
w_{t}=x_{t}+e_{t},
\end{array}
$$

para $t=1, \ldots, n$, sendo $g(\cdot)$ e $h(\cdot)$ funções de ligação logito e logarítmica. Consideramos $\alpha=0,4$, $\beta=0,08, \gamma=3,48, \lambda=0,01$, admitimos que $x_{t} \sim N(4,37 ; 2,05)$ e $e_{t} \sim N\left(0 ; \sigma_{e}^{2}\right)$. Atribuímos os seguintes valores a $\sigma_{e}^{2}: 0,05,0,5$ e 1,0 . Construímos gráficos normais de probabilidade com o objetivo de confrontar os quantis empíricos dos resíduos ponderados padronizados 2 com os quantis teóricos da distribuição normal padrão. Nas Figuras 4.1, 4.2 e 4.3 mostram-se os gráficos normais de probabilidade para os três métodos de estimação estudados no Capítulo 2.2, a saber: máxima verossimilhança aproximada, máxima pseudo-verossimilhança aproximada e calibração da regressão. A partir do estudo de simulação podemos concluir que o resíduo ponderado padronizado 2 na presença de erros de medida apresenta um comportamento adequado em relação à concordância com a distribuição normal padrão quando utilizamos os métodos de estimação por máxima verossimilhança aproximada e máxima pseudo-verossimilhança aproximada. A superioridade mostrada pelos métodos anteriormente mencionados independe do tamanho da amostra e dos valores atribuídos à variância do erro de medição. No entanto, para o caso do método de calibração da regressão, observa- 
se que, para tamanhos de amostra pequeno e variância do erro grande, existem alguns casos que não pertencem ao intervalo $[-3,3]$. Assim, encontramos um indício a mais do bom comportamento dos de métodos máxima verossimilhança aproximada e máxima pseudo-verossimilhança aproximada na estimação dos parâmetros.

\subsubsection{Envelope simulado}

Atkinson (1985) sugere que, para poder interpretar melhor o gráfico normal de probabilidades do resíduo proposto, este deve ser auxiliado pela construção de envelopes. Os envelopes simulados são bandas de confiança obtidas pelo método de Monte Carlo a partir do modelo ajustado para avaliar a existência de afastamentos sérios da distribuição proposta. Em um gráfico de probabilidade meionormal, dispomos o $t$-ésimo $(t=1, \ldots, n)$ valor ordenado dos resíduos versus os valores esperados das estatísticas de ordem, em valor absoluto, da distribuição normal padrão $N(0,1)$, dados por

$$
\Phi^{-1}\left(\frac{t+n-1 / 8}{2 n+1 / 2}\right),
$$

sendo $\Phi(\cdot)$ a função de distribuição acumulada da distribuição $N(0,1)$. Esse gráfico pode ser utilizado mesmo que os resíduos não tenham distribuição de probabilidade normal. Quando isso ocorre, não esperamos que os mesmos estejam próximos da reta identidade. A seguir apresentamos os passos para a construção do gráfico meio-normal com envelope simulado:

1. ajuste o modelo da amostra original e calcular o resíduo proposto;

2. gere uma amostra simulada de $n$ observações independentes utilizando o modelo ajustado;

3. ajuste o modelo para a amostra gerada, e calcule os valores ordenados dos resíduos ponderados padronizados 2 ;

4. repita os passos (1) e (2) $R$ vezes;

5. do grupo de $R$ valores de resíduos obtidos, calcule suas respectivas médias, valores mínimos e máximos;

6. construa um gráfico desses valores e do resíduo ordenado da amostra original contra percentis esperados da distribuição normal padrão.

Os valores mínimos e máximos das $R$ réplicas produzem o envelope. Segundo Atkinson (1985), é suficiente usar $R=19$. Deste modo, a probabilidade de qualquer resíduo em valor absoluto de um envelope exceder o limite superior fica sendo aproximadamente igual a $1 / 20=0,05$.

\subsection{Análise de influência global}

Esta metodologia consiste em estudar o efeito de retirar a t-ésima observação de um conjunto de dados. Seja uma variável aleatória contínua y com logaritmo da função de verossimilhança com parâmetro $\boldsymbol{\Psi}$ denotado por $\ell(\boldsymbol{\Psi})$. Seja $\hat{\boldsymbol{\Psi}}_{(t)}$ o estimador de $\boldsymbol{\Psi}$ utilizado, em que o subscrito $(t)$ indica que a $t$-ésima observação foi eliminado. A influência do t-ésimo indivíduo eliminado no estimador de $\hat{\mathbf{\Psi}}$ é avaliada pela diferença entre $\widehat{\mathbf{\Psi}}_{(t)}$ e $\widehat{\boldsymbol{\Psi}}$.

Se a eliminação de uma observação provoca variações significativas nas estimativas, mais atenção deve ser dada para essa observação. Se $\widehat{\Psi}_{(t)}$ está distante de $\widehat{\boldsymbol{\Psi}}$, então, o t-ésimo indivíduo eliminado 

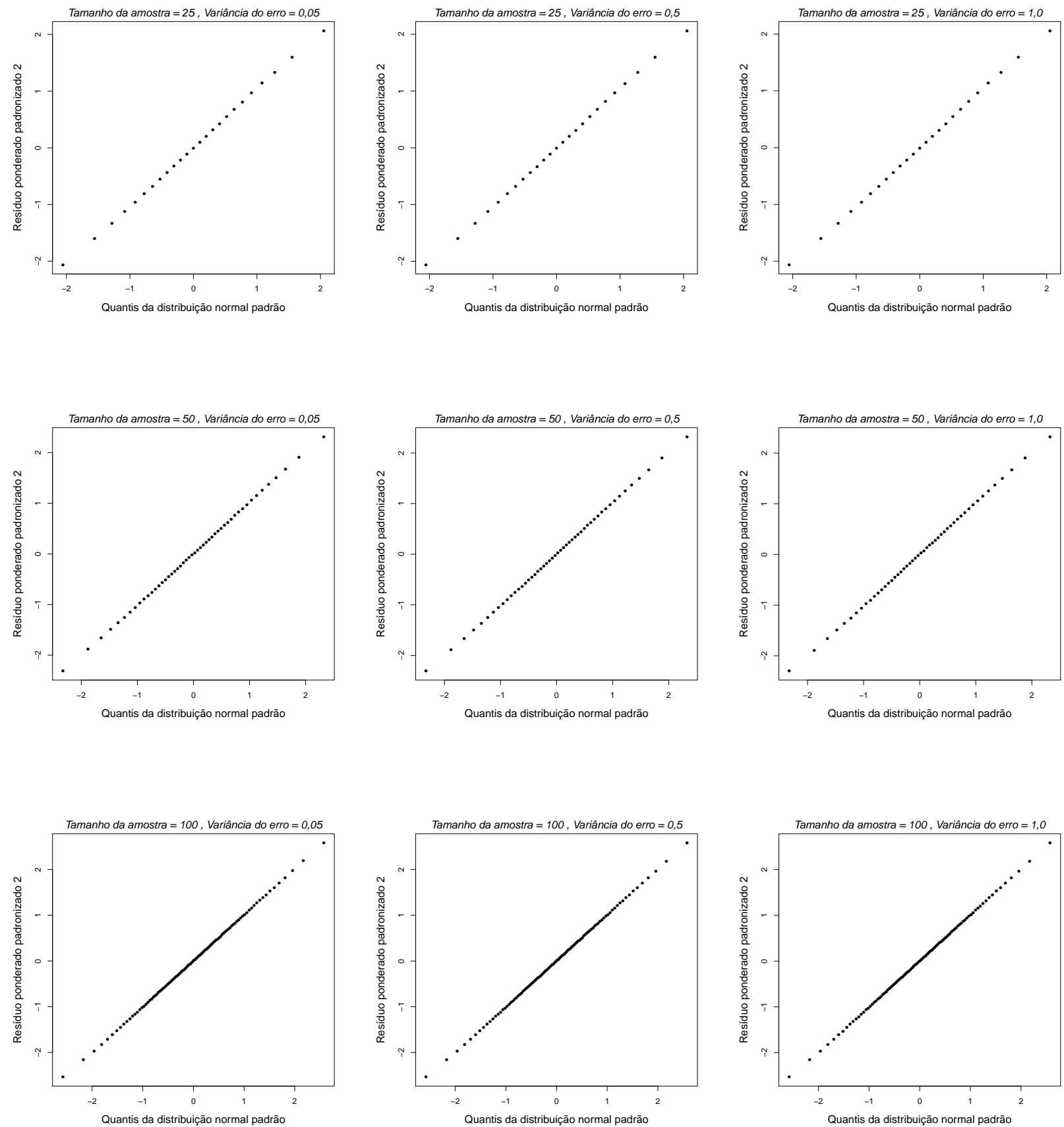

Figura 4.1: Gráficos normais de probabilidade para os resíduos ponderados padronizados 2 obtidos utilizando o método de máxima verossimilhança para estimar os parâmetros 

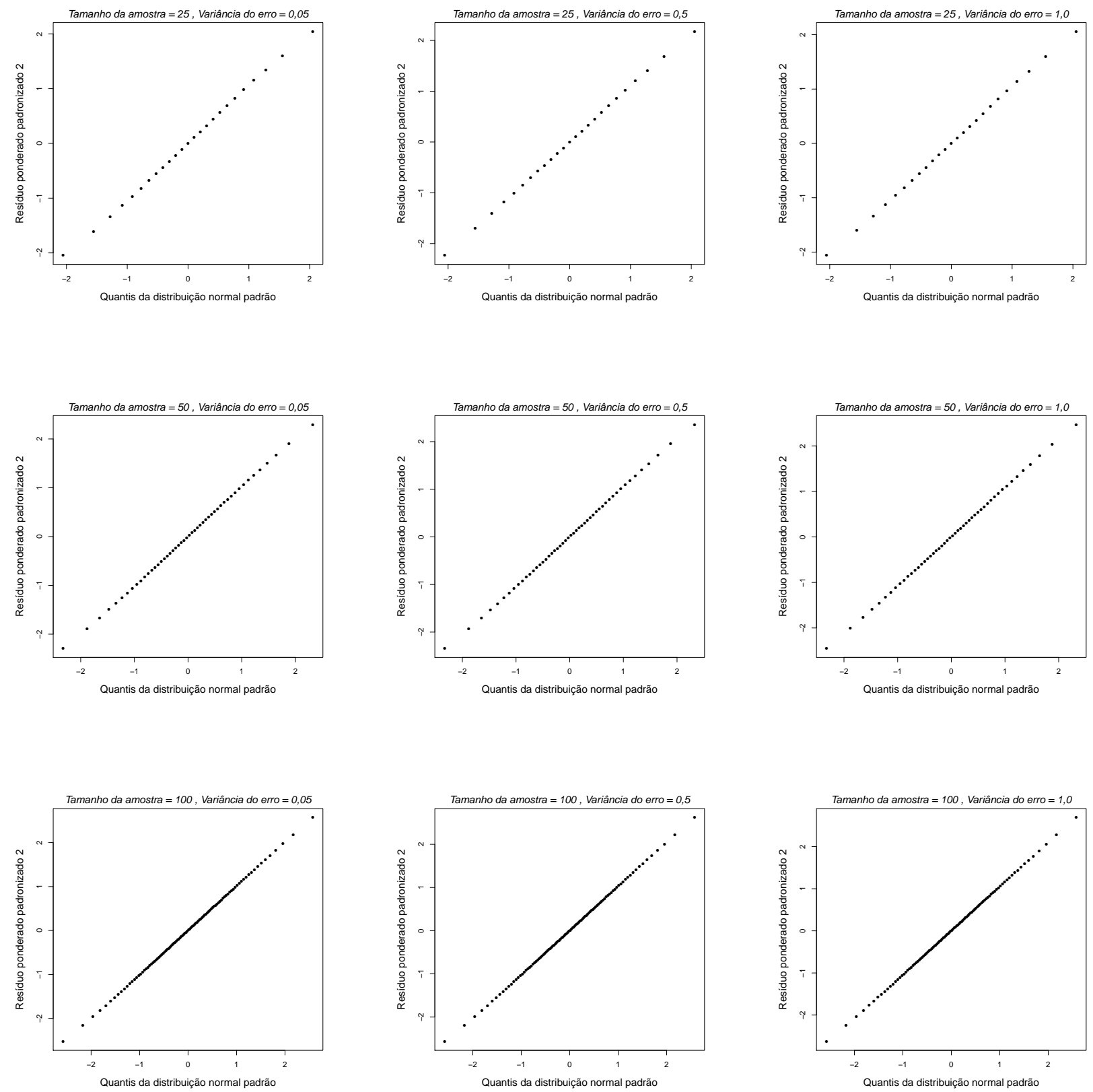

Figura 4.2: Gráficos normais de probabilidade para os resíduos ponderados padronizados 2 obtidos utilizando o método de máxima pseudo-verossimilhança aproximada para estimar os parâmetros 

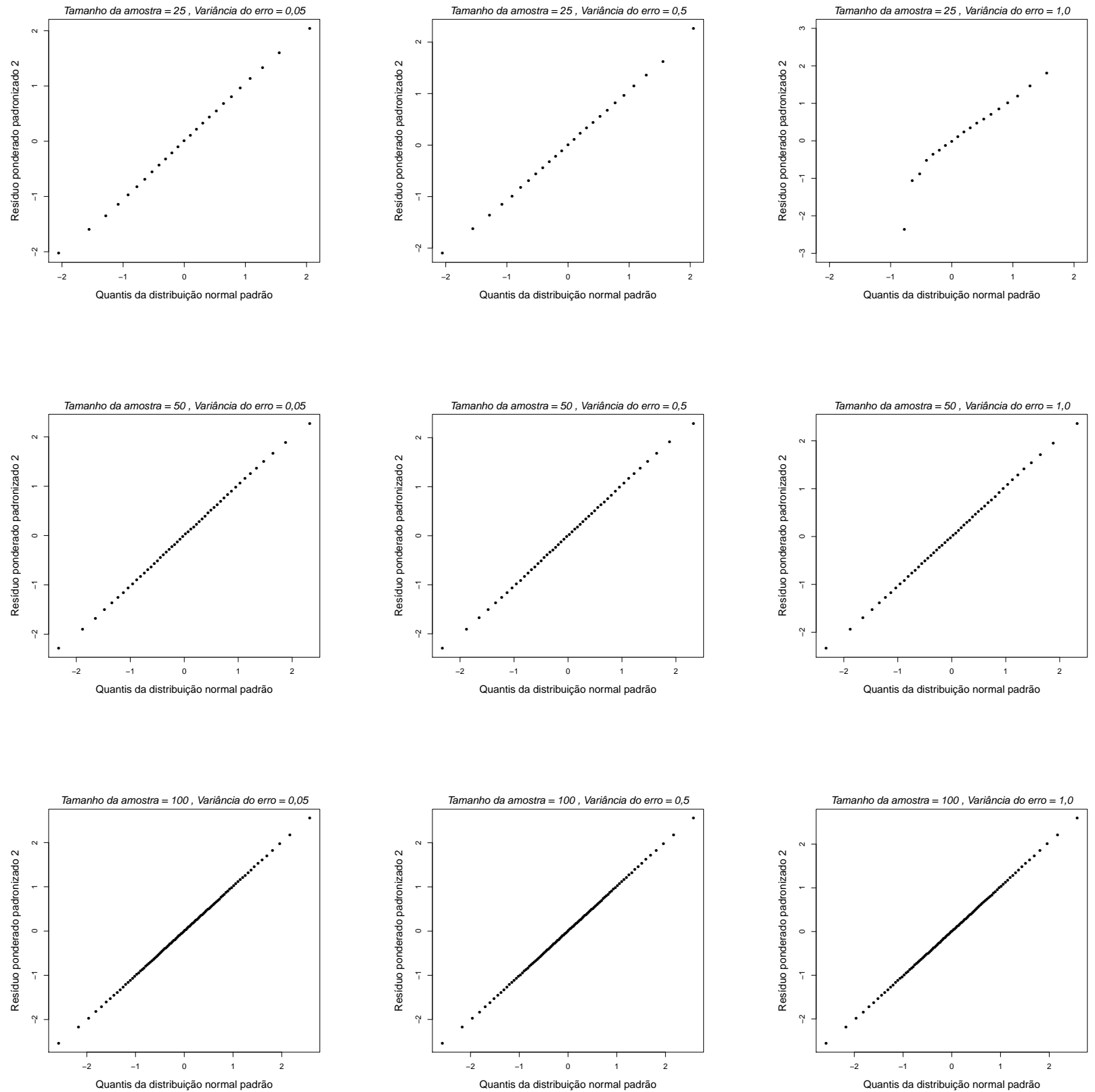

Figura 4.3: Gráficos normais de probabilidade para os resíduos ponderados padronizados 2 obtidos utilizando o método de calibração da regressão para estimar os parâmetros 
é considerado como uma observação influente. Uma primeira medida é definida como a norma padronizada de $\widehat{\Psi}_{(t)}-\widehat{\Psi}$, e é conhecida também como a distância de Cook generalizada, definida por

$$
C D_{t}(\mathbf{\Psi})=\left(\widehat{\mathbf{\Psi}}_{(t)}-\widehat{\mathbf{\Psi}}\right)^{\top}[-\ddot{\ell}(\mathbf{\Psi})]\left(\widehat{\mathbf{\Psi}}_{(t)}-\widehat{\mathbf{\Psi}}\right) .
$$

Podemos utilizar também uma outra medida para verificar a existência de pontos influentes. Esta medida é conhecida na literatura como afastamento da verossimilhança, que é dada por

$$
L D_{t}(\mathbf{\Psi})=2\left\{\ell(\widehat{\mathbf{\Psi}})-\ell\left(\widehat{\boldsymbol{\Psi}}_{(t)}\right)\right\}
$$

Como as estimativas dos parâmetros do modelo de regressão beta com erros de medida podem ser obtidas a partir dos métodos tratados em (2.2), sugerimos substituir $\ell(\boldsymbol{\Psi})$ em (4.3) e (4.4), por $\ell_{a}(\cdot), \ell_{p}(\cdot)$ ou $\ell_{R C}(\cdot)$. Recorde que $\ell_{p}(\cdot)$ e $\ell_{R C}(\cdot)$ dependem unicamente do vetor de parâmetros de interesse.

Utilizamos a função dropr (Doornik, 2011, Pag. 114) da linguagem de programação Ox para eliminar a $t$-ésima observação. Os passos para encontrar as medidas globais de Cook generalizadas e afastamento da verossimilhança na linguagem de programação utilizada são dados no Apêndice B.8.

\subsection{Análise de influência local}

Os métodos de diagnóstico visam a verificação de possíveis afastamentos das suposições formuladas para o modelo, bem como a identificação da existência de observações extremas. Dentre os métodos que auxiliam a detectar observações extremas, destacamos a influência local proposta por Cook (1986). Este método consiste em verificar a existência de pontos que, sob pequenas modificações (perturbações), causam variações significativas nos resultados do ajuste. O método sugerido por Cook (1986) baseia-se na função denominada afastamento pela verossimilhança, definida por

$$
L D(\boldsymbol{\delta})=2\left[\ell(\widehat{\mathbf{\Psi}})-\ell\left(\widehat{\mathbf{\Psi}}_{\delta}\right)\right]
$$

em que $\ell(\cdot)$ é o logaritmo da função de verossimilhança correspondente ao modelo postulado ou modelo assumido correto, $\hat{\boldsymbol{\Psi}}$ e $\widehat{\boldsymbol{\Psi}}_{\delta}$ são estimadores de máxima verossimilhança em $\ell(\boldsymbol{\Psi})$ e no logaritmo da função de verossimilhança do modelo perturbado $\ell_{\delta}(\boldsymbol{\Psi})$, respectivamente, $\delta=\left(\delta_{1}, \delta_{2}, \ldots, \delta_{n}\right)^{\top}$ representa o vetor de perturbações de dimensão $n \times 1$, assumindo valores no conjunto aberto $\mathfrak{D}$. Suponhamos a existência de um vetor $\delta_{0} \in \mathfrak{D}$ de tal forma que $\ell\left(\boldsymbol{\Psi}_{\delta_{0}}\right)=\ell(\boldsymbol{\Psi})$, aqui $\delta_{0}$ representa a ausência de perturbação no modelo postulado.

A proposta de Cook (1986) é estudar o comportamento local de $L D(\delta)$ em torno de $\delta_{0}$, que consiste em avaliar como a superfície ${ }^{1}$ geométrica

$$
\check{\alpha}(\delta)=\left(\delta_{1}, \delta_{2}, \ldots, \delta_{n}, L D(\delta)\right)^{\top}
$$

desvia-se de seu plano tangente em $\boldsymbol{\delta}_{0}$ à medida que $\boldsymbol{\delta}$ se afasta lentamente de $\boldsymbol{\delta}_{0}$, ou seja, quando pequenas perturbações são introduzidas ao modelo. Essa análise é feita através do estudo das curvaturas de secções normais da superfície $\check{\alpha}(\delta)$ ao redor de $\delta_{0}$ numa direção d. Cook (1986)

\footnotetext{
${ }^{1}$ A superfície é chamada de gráfico de influência.
} 
mostra que a curvatura de secções normais, $C_{\mathbf{d}}(\boldsymbol{\Psi})$, numa direção $\mathbf{d}$ é dada por

$$
C_{\mathbf{d}}(\Psi)=2\left|\mathbf{d}^{\top} \ddot{F}(\Psi) \mathbf{d}\right|
$$

em que $\ddot{F}(\boldsymbol{\Psi})=\Delta^{\top}[-\ddot{\ell}(\boldsymbol{\Psi})]^{-1} \Delta, \ddot{\ell}(\boldsymbol{\Psi})=\partial^{2} \ell(\boldsymbol{\Psi}) / \partial \boldsymbol{\Psi} \partial \boldsymbol{\Psi}^{\top}, \Delta=\partial^{2} \ell_{\delta}(\boldsymbol{\Psi}) / \partial \delta \partial \boldsymbol{\Psi}^{\top}$ é uma matriz de dimensão $n(\boldsymbol{\Psi}) \times n$ avaliadas em $\boldsymbol{\Psi}=\hat{\boldsymbol{\Psi}}$ e $\delta=\delta_{0}$, sendo que $n(\boldsymbol{\Psi})$ representa a dimensão de $\boldsymbol{\Psi}$. Pode-se expressar $C_{\mathbf{d}}(\boldsymbol{\Psi})$ como

$$
C_{\mathbf{d}}(\boldsymbol{\Psi})=2 \sum_{m=1}^{n} \lambda_{m} \mathbf{v}_{m} \mathbf{v}_{m}^{\top}
$$

em que $\lambda_{1} \geq \lambda_{2} \geq \ldots \geq \lambda_{n(\boldsymbol{\Psi})} \geq \lambda_{n(\boldsymbol{\Psi})+1} \geq \ldots \geq \lambda_{n}$ são os autovalores ordenados da matriz $\ddot{F}(\boldsymbol{\Psi})$ e $\mathbf{v}_{1}, \mathbf{v}_{2}, \ldots, \mathbf{v}_{n}$ são seus respectivos autovetores. O interesse está na direção que produz a maior influência local. Essa direção, $\mathbf{d}_{\max }$, é o autovetor normalizado correspondente ao maior autovalor de $\ddot{F}(\boldsymbol{\Psi})$. O gráfico de componentes do autovetor $\mathbf{d}_{\max }$ versus o índice das observações é útil para identificar observações influentes.

Se o interesse é avaliar a influência local em parte do vetor de parâmetros $\boldsymbol{\Psi}=\left(\boldsymbol{\theta}^{\top}, \boldsymbol{\xi}^{\top}, \boldsymbol{\sigma}_{e}^{2 \top}\right)^{\top}$, como por exemplo o vetor de parâmetros de interesse, o afastamento da verossimilhança (4.5) é dado por

$$
L D_{\boldsymbol{\theta}}(\boldsymbol{\delta})=2\left\{\ell(\widehat{\boldsymbol{\theta}}, \widehat{\boldsymbol{\xi}})-\ell\left(\widehat{\boldsymbol{\theta}}_{\delta}, \ell^{*}\left(\widehat{\boldsymbol{\theta}}_{\delta}\right)\right)\right\}
$$

em que $\ell^{*}\left(\widehat{\boldsymbol{\theta}}_{\delta}\right)$ é uma função que maximiza $\ell(\boldsymbol{\theta}, \boldsymbol{\xi})$ para cada $\boldsymbol{\theta}$ fixo, $\widehat{\boldsymbol{\theta}}_{\delta}$ é obtida de $\left(\widehat{\boldsymbol{\theta}}_{\delta}, \widehat{\boldsymbol{\xi}}_{\delta}\right)^{\top}$ e $\ell\left(\boldsymbol{\theta}_{\rho}, \ell^{*}\left(\boldsymbol{\theta}_{\delta}\right)\right)$ é o logaritmo da função de verossimilhança perfilada para $\boldsymbol{\theta}$. A curvatura normal na direção do vetor $\mathbf{d}$ fica dada por

$$
C_{\mathbf{d}}(\boldsymbol{\theta})=2\left|\mathbf{d}^{\top} \Delta^{\top}\left\{[\ddot{\ell}(\Psi)]^{-1}-\mathbf{D}_{1}\right\} \Delta \mathbf{d}\right|,
$$

em que

$$
\begin{aligned}
\mathbf{D}_{1} & =\left[\begin{array}{cc}
0 & 0 \\
0 & {[\ddot{\ell}(\boldsymbol{\xi})]^{-1}}
\end{array}\right], \\
\ddot{\ell}(\boldsymbol{\xi}) & =\left.\frac{\partial^{2} \ell_{\delta}(\boldsymbol{\Psi})}{\partial \boldsymbol{\xi}^{\top} \partial \boldsymbol{\xi}}\right|_{\boldsymbol{\Psi}=\widehat{\boldsymbol{\Psi}}} .
\end{aligned}
$$

O gráfico do autovetor associado ao maior autovalor da matriz $-\Delta^{\top}\left\{[\ddot{\ell}(\boldsymbol{\theta})]^{-1}-\mathbf{D}_{1}\right\} \Delta$ versus os índices de observações pode revelar quais destas estão influenciando $\widehat{\boldsymbol{\theta}}$. Recorde que a variância do erro de medida é conhecida ou estimada se há réplicas de $w_{t}$.

Lesaffre e Verbeke (1998) sugerem considerar a direção da t-ésima observação, que é o vetor $\mathbf{d}_{t}=(0, \ldots, 1, \ldots, 0)$, sendo que o $t$-ésimo elemento é um. Nesse sentido, a curvatura normal chamada de influência local total do t-ésimo indivíduo é dada por

$$
C_{\mathbf{d}, t}(\boldsymbol{\Psi})=2\left|\Delta_{t}^{\top}[-\ddot{\ell}(\mathbf{\Psi})]^{-1} \Delta_{t}\right|
$$

em que $\Delta_{t}$ denota a $t$-ésima linha da matriz $\Delta$. Os autores sugerem estudar o gráfico de $C_{\mathbf{d}, t}(\Psi)$ 
contra a ordem das observações para detectar pontos influentes. É sugerido também pelos autores utilizar como ponto de corte nos gráficos de $C_{\mathbf{d}, t}(\Psi)$, duas vezes o valor médio desta medida, ou seja, observações com $C_{\mathbf{d}, t}(\Psi)$ maiores que $2 n^{-1} \sum_{t=1}^{n} C_{\mathbf{d}, t}(\Psi)$ podem ser classificadas como influentes.

O método proposto por Cook (1986) apresenta alguns inconvenientes. Por exemplo, não é invariante sob reparametrizações de escala. Para contornar esse inconveniente, Poon e Poon (1999) definem a curvatura normal conforme no ponto $\delta_{0}$ na direção $\mathbf{d}$ como

$$
B_{\mathbf{d}}(\boldsymbol{\Psi})=\frac{2\left|\mathbf{d}^{\top} \Delta^{\top}[\ddot{\ell}(\Psi)]^{-1} \Delta \mathbf{d}\right|}{\sqrt{\operatorname{tr}\left(A^{\top} A\right)}},
$$

em que $A=2 \Delta^{\top}[\ddot{\ell}(\Psi)]^{-1} \Delta$. Notemos que o cálculo da expressão (4.8) não requer mais esforço que o de $C_{\mathbf{d}}(\boldsymbol{\Psi})$. Para qualquer direção $\mathbf{d}, B_{\mathbf{d}}(\boldsymbol{\Psi})$ satisfaz a condição $0<B_{\mathbf{d}}(\boldsymbol{\Psi})<1$, e desta forma, $B_{\mathbf{d}}(\Psi)$ é uma medida normalizada, o que torna mais fácil a interpretação de seu valor. $\mathrm{O}$ valor da curvatura normal conforme $B_{\mathbf{d}}(\boldsymbol{\Psi})$ é igual ao autovalor normalizado

$$
\hat{\lambda}_{t}=\frac{\lambda_{t}}{\sqrt{\sum_{t=1}^{n} \lambda_{t}^{2}}},
$$

sendo que $\sum_{t=1}^{n} B_{\mathbf{v}_{t}}^{2}(\boldsymbol{\Psi})=1$. Se a curvatura normal conforme é a mesma para todos os autovetores, então o valor comum é $1 / \sqrt{n}$ e este pode ser usado como referência para decidir se uma observação é influente ou não (Poon e Poon, 1999). Poon e Poon (1999) sugerem a utilização de um valor de referência para comparar os efeitos de $B_{\mathbf{v}_{t}}(\mathbf{\Psi}), t=1, \ldots, n$ e $B_{\mathbf{d}_{\text {max }}}(\mathbf{\Psi})$ em vários níveis, dando a seguinte definição.

Definição 4.4.1. Um autovetor $\mathbf{v}_{t}$ será $q$-influente se $\left|B_{\mathbf{v}_{t}}(\mathbf{\Psi})\right| \geq q / \sqrt{n}$.

Escolhemos valores para $q \in \mathbb{R}^{+}$; uma direção é influente de acordo com a magnitude de seu correspondente autovalor normalizado. De forma geral, se tomamos o módulo dos autovalores normalizados, $\lambda_{t}^{*}=\left|\hat{\lambda}_{t}\right|$, de forma que

$$
\lambda_{\max }=\lambda_{t}^{*} \geq \ldots \geq \lambda_{k}^{*} \geq q / \sqrt{n}>\lambda_{k+1}^{*} \ldots \lambda_{n}^{*} \geq 0,
$$

e denotamos por $a_{t j}$ a $j$-ésima coordenada do autovetor correspondente a $\lambda_{t}^{*}$, podemos obter a contribuição agregada do $j$-ésimo vetor básico para todos os autovetores $q$-influentes em $\mathbb{R}^{n}$ ao tomar

$$
m[q]_{j}=\sqrt{\sum_{t=1}^{k} \lambda_{t}^{*} a_{t j}^{2}}
$$

Para interpretar a contribuição agregada, podemos construir um gráfico dos valores de $m[q]_{j}$ contra os índices $j=1, \ldots, n$ e verificar se existem pontos que se distanciam dos demais.

Dado que $\sum_{j=1}^{n} m[q]_{j}^{2}=\sum_{t=1}^{k} \lambda_{t}^{*}$, temos que a contribuição de todos os vetores de perturbação básicos é a mesma, então cada uma é igual a $\bar{m}[q]=\sqrt{n^{-1} \sum_{t=1}^{k} \lambda_{i}^{*}}$. Desta forma, para determinar a magnitude da contribuição individual dos vetores de perturbação básicos usamos $\bar{m}[q]$. Assim, podemos considerar dois casos. O primeiro é permitir que $q$ seja suficientemente grande para agregar somente o maior autovalor $\lambda_{\max }$. Nesse caso $\bar{m}[q]_{j}=\sqrt{\lambda_{\max }}\left|a_{1 j}\right|$ e este método é equivalente a 
comparar $\left|a_{1 j}\right| \operatorname{com} 1 / \sqrt{n}$. De outro lado, se admitimos que $q=0$, então todos os autovalores serão incluídos na análise. Aqui, $m[0]_{j}$ é dita ser a contribuição total, e escrevemos $m_{j}=\sqrt{\sum_{t=1}^{n} \lambda_{t}^{*} a_{t j}^{2}}$. Se a contribuição de todas as perturbações básicas for a mesma, então cada uma será igual a

$$
\bar{m}=\bar{m}[0]=\sqrt{n^{-1} \sum_{t=1}^{n} \lambda_{t}^{*}}=\sqrt{n^{-1} \sum_{t=1}^{n}\left|\lambda_{t}\right| / \sum_{t=1}^{n} \lambda_{t}^{2}} .
$$

A contribuição total $m_{j}$ e a curvatura normal conforme $B_{E_{j}}(\Psi)$ do vetor de perturbação básico ${ }^{2}$ $E_{j}$ estão altamente relacionadas. Se todos os autovalores são não negativos, $B_{E_{j}}(\Psi)$ é igual ao quadrado da contribuição total do $j$-ésimo vetor de perturbação básico. Ou seja, dado que a matriz $-\ddot{F}$ é semi-definida positiva e todos os autovalores são não negativos, temos que

$$
B_{E_{j}}(\Psi)=m_{j}^{2}
$$

para todo $j$. Agora, se a contribuição total de todos os $B_{E_{j}}(\Psi)$ é a mesma, então cada uma é igual a

$$
b=\frac{\operatorname{tr}[\ddot{F}(\Psi)]}{n \sqrt{\operatorname{tr}\left[\ddot{F}(\Psi)^{2}\right]}} .
$$

Para decidir se um determinado ponto é influente ou não, considerando a contribuição agregada dos $k$ autovalores que são maiores do que $q / \sqrt{n}$, é utilizada a contribuição média $\bar{m}[q]_{j}$, e para decidir se um caso é ou não influente, utilizamos o valor $b$ dado em (4.10). Poon e Poon (1999) propõem utilizar $\sqrt{2} \bar{m}[q]$ como ponto de corte para a contribuição agregada dos $k$ autovalores e $2 b$ como ponto de corte da contribuição agregada de todos os autovalores.

Outro procedimento de diagnóstico baseado em influência local é proposto em Zhu e Zhang (2004). Esta proposta tem como objetivo avaliar modelos mal especificados para uma amostra e detectar pontos influentes. Os autores observam também que a medida de influência para detetar pontos influentes utilizada por Lesaffre e Verbeke (1998) e Steen et al. (2001), dada por

$$
C_{\mathbf{d}, t}(\Psi)=2 \sum_{m=1}^{n(\boldsymbol{\Psi})} \lambda_{m} v_{t m}^{2}
$$

é pouco explorada, apresentando, assim, algumas propriedades para $C_{\mathbf{d}, t}(\boldsymbol{\Psi})$. Uma das propriedades destacadas por Zhu e Zhang (2004) com respeito a $C_{\mathbf{d}, t}(\boldsymbol{\Psi})$ é a equivalência assintótica desta com as medidas de eliminação de casos vistas na Seção 4.3, isto é

$$
n C_{\mathbf{d}, t}(\mathbf{\Psi})=2 n C D_{t}(\boldsymbol{\Psi})+o_{p}\left(n^{-1 / 2}\right)=2 n L D_{t}(\mathbf{\Psi})+o_{p}\left(n^{-1 / 2}\right)
$$

Deixamos como pesquisa futura a verificação da má especificação do modelo aos dados tal como propõem Zhu e Zhang (2004). A seguir, apresentamos a análise de influência local para o modelo de regressão beta com erros de medida sob os seguintes esquemas de perturbação: perturbação de casos, perturbação da variável resposta e perturbação de uma covariável com e sem erros de medida.

\footnotetext{
${ }^{2}$ Poon e Poon (1999) definem $E_{j}, j$-ésimo vetor, como o vetor de perturbação básico do espaço de perturbações, cujo $j$-ésimo elemento é 1 e todos os restantes são iguais a 0.
} 


\subsection{Influência local no modelo de regressão beta com erros de medida}

Seja $\boldsymbol{\Psi}=\left(\boldsymbol{\theta}^{\top}, \boldsymbol{\xi}^{\top}, \sigma_{e}^{2 \top}\right)^{\top}$ o vetor de parâmetros do modelo de regressão beta com erros de medida, sendo que $\boldsymbol{\sigma}_{e}^{2 \top}$ pode ser conhecido ou estimável se há réplicas para w. Os elementos da matriz $\Delta=\Delta(\boldsymbol{\theta}, \boldsymbol{\xi})=\left(\Delta_{\boldsymbol{\theta}}^{\top}, \Delta_{\boldsymbol{\xi}}^{\top}\right)^{\top}$ de dimensão $n(\boldsymbol{\Psi}) \times n$, para calcular as secções normais, são dados por $\Delta_{\boldsymbol{\theta}}=\partial^{2} \ell(\boldsymbol{\Psi}) / \partial \boldsymbol{\theta} \partial \delta^{\top}$ e $\Delta_{\boldsymbol{\xi}}=\partial^{2} \ell(\boldsymbol{\Psi}) / \partial \boldsymbol{\xi} \partial \delta^{\top}$, sendo estas avaliadas em $\widehat{\boldsymbol{\Psi}}=\left(\widehat{\boldsymbol{\theta}}^{\top}, \widehat{\boldsymbol{\xi}}^{\top}\right)^{\top}$ e $\delta=\delta_{0}$. Derivamos as curvaturas das seções normais para o modelo de regressão beta com erros de medida considerando os esquemas de perturbação mencionados acima. Para exemplificar esta metodologia restringimos o estudo ao caso em que os parâmetros são estimados via máxima pseudo-verossimilhança aproximada, dado que este método mostrou indícios de superioridade computacional e analítica em relação aos outros métodos.

\subsubsection{Ponderação de casos}

O logaritmo da função pseudo-verossimilhança perturbada, quando apresenta uma covariável medida com erro, é dada por

$$
\ell_{p \delta}\left(\boldsymbol{\theta} ; \widehat{\boldsymbol{\xi}}, \widehat{\sigma}_{e}^{2}\right)=\sum_{t=1}^{n} \delta_{t} \ell_{1 t}\left(\widehat{\boldsymbol{\xi}}, \widehat{\sigma}_{e}^{2}\right)+\sum_{t=1}^{n} \delta_{t} \ell_{2 t}\left(\boldsymbol{\theta}, \widehat{\boldsymbol{\xi}}, \widehat{\sigma}_{e}^{2}\right)
$$

em que $\ell_{1 t}\left(\widehat{\boldsymbol{\xi}}, \widehat{\sigma}_{e}^{2}\right)$ e $\ell_{2 t}\left(\boldsymbol{\theta}, \widehat{\boldsymbol{\xi}}, \widehat{\sigma}_{e}^{2}\right)$ são dadas em (2.17) e (2.18), $\widehat{\sigma}_{e}^{2}$ é o valor conhecido de $\sigma_{e}^{2}$ ou é a estimativa de $\sigma_{e}^{2}$ obtida de réplicas de $w_{t}, \widehat{\boldsymbol{\xi}}$ obtida a partir de $(2.15)$ e $\delta_{t} \in[0,1]$. Neste esquema de perturbação o vetor de não perturbação $\delta_{0}$ é dado pelo vetor de uns de dimensão $n \times 1$. A matriz $\Delta(\boldsymbol{\theta}, \widehat{\boldsymbol{\xi}})$ é dada por

$$
\begin{aligned}
\Delta(\boldsymbol{\theta}, \widehat{\boldsymbol{\xi}}) & =\left.[\Delta(\boldsymbol{\alpha}), \Delta(\beta), \Delta(\boldsymbol{\gamma}), \Delta(\lambda)]^{\top}\right|_{\boldsymbol{\theta}=\widehat{\boldsymbol{\theta}}_{, \delta=\delta_{0},},} \\
& =\left.\left[A_{\boldsymbol{\alpha}}^{\top} B, A_{\beta}^{\top} B, A_{\boldsymbol{\gamma}}^{\top} B, A_{\lambda}^{\top} B\right]^{\top}\right|_{\boldsymbol{\theta}=\widehat{\boldsymbol{\theta}}, \delta=\delta_{0},}
\end{aligned}
$$

em que as expressões de $A_{\boldsymbol{\alpha}}^{\top}, A_{\beta}^{\top}, A_{\boldsymbol{\gamma}}^{\top}, A_{\lambda}^{\top}$ e $B$ encontram-se no Apêndice A.2.1, sendo que as estimativas de $\mu_{x}$ e $\sigma_{x}^{2}$ são obtidas a partir de (2.15).

\subsubsection{Perturbação da variável resposta}

Consideramos o esquema aditivo de perturbação da variável resposta em que $y_{1}, y_{2}, \ldots, y_{n}$ são transformados através da adição de um vetor de pequenas perturbações da forma

$$
y_{t}^{\dagger}=y_{t}+\delta_{t} S_{y}
$$

em que $S_{y}$ é um fator de escala dado pela estimativa do desvio padrão da variável $y_{t}$, isto é, $S_{y}=$ $\sqrt{\widehat{\mu}_{t}\left(1-\widehat{\mu}_{t}\right) /\left(1+\widehat{\phi}_{t}\right)}$, o vetor de não perturbação é dado por $\delta_{0}=(0, \ldots, 0)^{\top}$. Assim, o logaritmo da função pseudo-verossimilhança do modelo perturbado quando temos uma única covariável medida com erro é dado por

$$
\ell_{p \delta}\left(\boldsymbol{\theta} ; \widehat{\boldsymbol{\xi}}, \widehat{\sigma}_{e}^{2}\right)=\sum_{t=1}^{n} \ell_{1 t}\left(\widehat{\boldsymbol{\xi}}, \widehat{\sigma}_{e}^{2}\right)+\sum_{t=1}^{n} \ell_{2 t}^{\dagger}\left(\boldsymbol{\theta}, \widehat{\boldsymbol{\xi}}, \widehat{\sigma}_{e}^{2}\right)
$$


em que

$$
\begin{aligned}
\ell_{2 t}^{\dagger}\left(\boldsymbol{\theta}, \widehat{\boldsymbol{\xi}}, \widehat{\sigma}_{e}^{2}\right)= & \log \Gamma\left(\phi_{t}\right)-\log \Gamma\left(\mu_{t} \phi_{t}\right)-\log \Gamma\left[\left(1-\mu_{t}\right) \phi_{t}\right]+\left(\mu_{t} \phi_{t}-1\right) \log \left(y_{t}^{\dagger}\right) \\
& +\left[\left(1-\mu_{t}\right) \phi_{t}-1\right] \log \left(1-y_{t}^{\dagger}\right) .
\end{aligned}
$$

A matriz $\Delta(\boldsymbol{\theta}, \widehat{\boldsymbol{\xi}})$ neste esquema é dada por

$$
\begin{aligned}
\Delta(\boldsymbol{\theta}, \widehat{\boldsymbol{\xi}}) & =\left.[\Delta(\boldsymbol{\alpha}), \Delta(\beta), \Delta(\boldsymbol{\gamma}), \Delta(\lambda)]^{\top}\right|_{\boldsymbol{\theta}=\widehat{\boldsymbol{\theta}}, \delta=\delta_{0},} \\
& =\left.\left[\frac{\partial^{2} \ell_{p \delta}(\boldsymbol{\theta})}{\partial \delta \partial \boldsymbol{\alpha}^{\top}}, \frac{\partial^{2} \ell_{p \delta}(\boldsymbol{\theta})}{\partial \delta \partial \beta}, \frac{\partial^{2} \ell_{p \delta}(\boldsymbol{\theta})}{\partial \delta \partial \boldsymbol{\gamma}^{\top}}, \frac{\partial^{2} \ell_{p \delta}(\boldsymbol{\theta})}{\partial \boldsymbol{\delta} \partial \lambda}\right]^{\top}\right|_{\boldsymbol{\theta}=\widehat{\boldsymbol{\theta}}_{, \delta=\delta_{0}}}
\end{aligned}
$$

Observe que estes esquemas de perturbação pode facilmente ser adaptado quando temos mais covariáveis medidas com erro.

\subsubsection{Perturbação de covariáveis}

Aqui consideremos um esquema de perturbação aditivo de uma covariável contínua. A covariável selecionada para ser perturbada é transformada através do incremento de um vetor de perturbação vezes a estimativa do desvio padrão da variável perturbada. No casso em que o parâmetro de precisão e a média da variável resposta são modelados simultaneamente, mediante as expressões (2.4) e (2.5), consideramos aqui os seguintes esquemas de perturbação:

- quando assumimos simultaneamente que $\mathbf{z}_{t}^{\top} \neq \mathbf{v}_{t}^{\top}$ e $\mathbf{x}_{t}^{\top} \neq \mathbf{m}_{t}^{\top}$,

- quando $\mathbf{z}_{t}^{\top} \neq \mathbf{v}_{t}^{\top}$ e $\mathbf{x}_{t}^{\top}=\mathbf{m}_{t}^{\top}$,

- quando $\mathbf{z}_{t}^{\top}=\mathbf{v}_{t}^{\top}$ e $\mathbf{x}_{t}^{\top} \neq \mathbf{m}_{t}^{\top}$ e

- quando $\mathbf{z}_{t}^{\top}=\mathbf{v}_{t}^{\top}$ e $\mathbf{x}_{t}^{\top}=\mathbf{m}_{t}^{\top}$,

em que $\mathbf{z}_{t}$ e $\mathbf{x}_{t}$ são as matrizes de regressores, sem e com erros de medida, para o modelo da média e $\mathbf{v}_{t}$ e $\mathbf{m}_{t}$ da mesma forma são matrizes de regressores, sem e com erros de medida, para o modelo da precisão. O vetor de não perturbação é dado por $\boldsymbol{\delta}_{0}=(0, \ldots, 0)^{\top}$. Os elementos da matriz $\Delta$ poderiam ser determinados algebricamente, mas neste trabalho utilizamos técnicas numéricas para encontrar tais elementos.

O cálculo das seções normais para os diferentes esquemas de perturbação foram determinados numericamente utilizando a linguagem de programação ox, tal como mostra o Apêndice B.9. 


\section{Capítulo 5}

\section{Aplicações}

Este capítulo objetiva ilustrar as metodologias desenvolvidas nesta tese para o modelo de regressão beta com erros de medida. Consideramos aqui os conjuntos de dados referidos na Seção 1.1 .

\subsection{Aplicação I}

Consideramos o conjunto de dados referente à distribuição de gás natural da Comgás. A Comgás distribui gás natural atendendo a seis segmentos do mercado: residencial, comercial, industrial, automotivo, cogeração e termogeração. Consideraremos aqui, unicamente o segmento residencial para ilustrar as metodologias apresentadas.

Analisamos o conjunto de dados de forma que para cada unidade experimental foram observados o fator de carga, variável resposta $y_{t}$, e uma única covariável com aparente erro de medida, $w_{t}$, representada pelo logaritmo do consumo médio mensal registrado durante o ano 2004. O erro de medição na covariável está associado à imprecisão do aparelho (medidor de gás) no registro do consumo mensal de gás. Foi selecionada e monitorada uma amostra de 70 unidades (prédios). O período de monitoramento iniciou-se em dezembro do 2002 e finalizou em maio do 2003, considerando sete dias de monitoramento completos para cada unidade.

Inicialmente realizamos uma análise descritiva das variáveis envolvidas no problema. Na Tabela 5.1 mostramos algumas medidas resumo. Observamos que o fator de carga médio amostral é 0,0932. Notamos também uma acentuada assimetria da variável resposta. Em termos da covariável observamos que, em média, o consumo mensal de gás é aproximadamente $\exp (2,3930)=10,946 \mathrm{~m}^{3} / \mathrm{h}$. Construímos o gráfico de dispersão entre o fator de carga e logaritmo natural do consumo médio

Tabela 5.1: Medidas resumo das variáveis

\begin{tabular}{c|rcccccc}
\hline Variável & Mínimo & $Q_{1}$ & Mediana & Média & $Q_{3}$ & Máximo & Desvio padrão \\
\hline$y$ & 0,0098 & 0,0474 & 0,0671 & 0,0932 & 0,0965 & 0,6278 & 0,0967 \\
$\log ($ consumo $)$ & $-2,6300$ & 1,6725 & 2,1750 & 2,3930 & 3,0200 & 8,9700 & 1,6703 \\
\hline
\end{tabular}

anual, $\log ($ consumo $)$, com o objetivo de avaliar a relação entre as duas variáveis. A Figura 1.1 mostra uma clara relação entre estas duas variáveis, sugerindo um comportamento não linear. O gráfico sugere um crescimento exponencial à medida em que o consumo mensal aumenta. Mostra também a existência de cinco observações isoladas da massa de dados. A unidade $\sharp 1$ corresponde à residência com menor consumo médio anual e menor fator de carga e as unidades $\sharp 67, \sharp 68, \sharp 69$ e $\sharp 70$ correspondem às residenciais com maior consumo médio anual e maior fator de carga. Observa-se que o fator de carga resulta em valores no intervalo $(0,009 ; 0,627)$ e o consumo médio anual considerado 
como variável independente está associado a um erro de medição. Assim, é adequado considerar o modelo de regressão beta com erros de medida. Admitimos que $y_{1}, y_{2}, \ldots, y_{n}$ são variáveis aleatórias independentes tais que $y_{t}$, para todo $t=1, \ldots, n$, tem distribuição beta de média $\mu_{t}$ e parâmetro de precisão $\phi_{t}$, e ainda,

$$
\begin{aligned}
\log \left(\frac{\mu_{t}}{1-\mu_{t}}\right) & =\alpha+\beta x_{t}, \\
\log \left(\phi_{t}\right) & =\gamma+\lambda x_{t}, \\
w_{t} & =x_{t}+e_{t},
\end{aligned}
$$

em que $t=1, \ldots, n, x_{t} \sim N\left(\mu_{x}, \sigma_{x}^{2}\right)$ e $e_{t} \sim N\left(0, \sigma_{e}^{2}\right)$. Consideramos inicialmente um modelo em que a covariável $w_{t}$ é modelada pela média e a dispersão. A partir dos consumos mensais, doze medições para cada unidade amostral, é possível determinar a estimativa da variância do erro de medida, $\sigma_{e}^{2}$. Obtemos $\widehat{\sigma}_{e}^{2}=0,012\left(\mathrm{~m}^{3} / \mathrm{h}\right)^{2}$.

Determinamos as estimativas dos parâmetros a partir dos métodos de máxima verossimilhança aproximada, pseudo-verossimilhança aproximada, calibração da regressão e naïve. A Tabela 5.2 mostra as estimativas, erros padrão e níveis descritivos para os métodos anteriormente mencionados. Nesta tabela apresentamos também resultados inferenciais utilizando técnicas de reamostragem bootstrap não paramétrico. Mais detalhes sobre a técnica bootstrap não paramétrico utilizada nesta tese encontram-se no Apêndice A.4.

\begin{tabular}{|c|c|c|c|c|c|c|c|c|}
\hline \multirow{2}{*}{ Método } & \multirow[b]{2}{*}{$\widehat{\theta}$} & \multirow[b]{2}{*}{$E$} & \multirow[b]{2}{*}{$E P$} & \multirow[b]{2}{*}{$p$} & \multicolumn{4}{|c|}{ bootstrap } \\
\hline & & & & & $E$ & $E P$ & $p$ & $I C$ \\
\hline \multirow{4}{*}{$\ell_{a}$} & $\alpha$ & $-3,519$ & 0,087 & 0,000 & $-3,560$ & 0,089 & 0,000 & $(-3,662 ;-3,377)$ \\
\hline & $\beta$ & 0,428 & 0,028 & 0,000 & 0,422 & 0,032 & 0,000 & $(0,371 ; 0,471)$ \\
\hline & $\gamma$ & 5,097 & 0,358 & 0,000 & 5,282 & 0,606 & 0,000 & $(4,110 ; 5,976)$ \\
\hline & $\lambda$ & $-0,156$ & 0,136 & 0,250 & $-0,187$ & 0,250 & 0,190 & $(-0,578 ; 0,204)$ \\
\hline \multirow{4}{*}{$\ell_{p}$} & $\alpha$ & $-3,519$ & 0,090 & 0,000 & -3.513 & 0,082 & 0,000 & $(-3,652 ;-3,389)$ \\
\hline & $\beta$ & 0,428 & 0,034 & 0,000 & 0,423 & 0,031 & 0,000 & $(0,376 ; 0,469)$ \\
\hline & $\gamma$ & 5,097 & 0,552 & 0,000 & 5,277 & 0,650 & 0,000 & $(4,105 ; 6,067)$ \\
\hline & $\lambda$ & $-0,156$ & 0,241 & 0,517 & $-0,186$ & 0,273 & 0,200 & $(-0,634 ; 0,236)$ \\
\hline \multirow{4}{*}{$\ell_{R C}$} & $\alpha$ & $-3,516$ & 0,081 & 0,000 & $-3,506$ & 0,082 & 0,000 & $(-3,647 ;-3,391)$ \\
\hline & $\beta$ & 0,427 & 0,025 & 0,000 & 0,420 & 0,031 & 0,000 & $(0,377 ; 0,466)$ \\
\hline & $\gamma$ & 5,097 & 0,296 & 0,000 & 5,268 & 0,637 & 0,000 & $(4,131 ; 6,103)$ \\
\hline & $\lambda$ & $-0,164$ & 0,101 & 0,105 & $-0,192$ & 0,264 & 0,184 & $(-0,635 ; 0,222)$ \\
\hline \multirow{4}{*}{$\ell_{\text {Nave }}$} & $\alpha$ & $-3,511$ & 0,086 & 0,000 & -3.506 & 0,082 & 0,000 & $(-3,641 ;-3,381)$ \\
\hline & $\beta$ & 0,425 & 0,027 & 0,000 & 0.421 & 0,031 & 0,000 & $(0,372 ; 0,465)$ \\
\hline & $\gamma$ & 5,095 & 0,345 & 0,000 & 5.263 & 0,635 & 0,000 & $(4,123 ; 6,096)$ \\
\hline & $\lambda$ & $-0,163$ & 0,128 & 0,205 & -0.189 & 0,265 & 0,184 & $(-0,634 ; 0,222)$ \\
\hline
\end{tabular}

Tabela 5.2: Estimativas (E), erros padrão (EP), p-valores (p) e intervalos de confiança (IC) com coeficiente de confiança de $95 \%$ obtidos pelos métodos dados na Seção 2.2 e bootstrap.

Observamos na Tabela 5.2 que as estimativas dos parâmetros obtidas por máxima verossimilhança aproximada, pseudo-verossimilhança aproximada, calibração da regressão e naïve são muito 
próximas. Resultados semelhantes foram obtidos quando utilizamos o método de reamostragem bootstrap. Em termos do erro padrão, o método de máxima pseudo-verossimilhança apresentou um ligeiro incremento frente aos demais. Observamos também que a covariável medida com erro, $w_{t}$, resultou como não significativa quando é usada para modelar o parâmetro de precisão. De fato, os $p$-valores para os testes de $\mathcal{H}_{0}: \lambda=0$ versus $\mathcal{H}_{1}: \lambda \neq 0$ baseados nas estimativas de $\lambda$ mostrados na Tabela 5.3 são todos superiores a 10\%. Portanto, concluímos que não existem evidências para rejeitar $\mathcal{H}_{0}$ aos níveis de significância usuais.

Tabela 5.3: Testes de $\mathcal{H}_{0}: \lambda=0$ baseados nas estatísticas da razão de verossimilhanças e gradiente (p-valores entre parênteses).

\begin{tabular}{c|rrrr}
\hline Teste & $\ell_{a}$ & $\ell_{p}$ & $\ell_{R C}$ & $\ell_{\text {Naive }}$ \\
\hline Razão de verossimilhanças & 1,396 & 0,902 & 1,703 & 1,703 \\
& $(0,236)$ & $(0,825)$ & $(0,636)$ & $(0,192)$ \\
Gradiente & 1,440 & 0,930 & 1,765 & 1,765 \\
& $(0,230)$ & $(0,818)$ & $(0,622)$ & $(0,184)$ \\
\hline
\end{tabular}

Consideramos agora o modelo de regressão beta com erros de medida em que o parâmetro de precisão é constante. Mostramos na Tabela 5.4 os resultados inferenciais. Nesta tabela pode-

Tabela 5.4: Estimativas (E), erros padrão (EP), p-valores (p) e intervalos de confiança (IC) com coeficiente de confiança de $95 \%$ obtidos pelos métodos dados na Seção 2.2 e bootstrap do modelo de regressão beta com erros de medida e dispersão constante.

\begin{tabular}{c|c|rrr|rrrr}
\cline { 5 - 8 } \multicolumn{1}{c}{} & \multicolumn{1}{c}{} & \multicolumn{1}{c}{ bootstrap } \\
\hline Método & $\widehat{\boldsymbol{\theta}}$ & \multicolumn{1}{c}{$E$} & $E P$ & \multicolumn{1}{c}{$p$} & \multicolumn{1}{c}{$E$} & \multicolumn{1}{c}{$E P$} & $p$ & \multicolumn{1}{c}{$I C$} \\
\hline \multirow{4}{*}{$\ell_{a}$} & $\alpha$ & $-3,545$ & 0,087 & 0,000 & $-3,560$ & 0,089 & 0,000 & $(-3,662 ;-3,377)$ \\
& $\beta$ & 0,438 & 0,028 & 0,000 & 0,422 & 0,032 & 0,000 & $(0,371 ; 0,471)$ \\
& $\phi$ & 112,366 & 20,493 & 0,000 & 126,261 & 40,901 & 0,002 & $(68,015 ; 187,227)$ \\
\hline \multirow{4}{*}{$\ell_{p}$} & $\alpha$ & $-3,545$ & 0,070 & 0,000 & $-3,539$ & 0,073 & 0,000 & $(-3,656 ;-3,425)$ \\
& $\beta$ & 0,438 & 0,018 & 0,000 & 0,435 & 0,023 & 0,000 & $(0,399 ; 0,467)$ \\
& $\phi$ & 112,377 & 30,187 & 0,001 & 119,327 & 36,321 & 0,000 & $(61,727 ; 165,348)$ \\
\hline \multirow{4}{*}{$\ell_{R C}$} & $\alpha$ & $-3,543$ & 0,077 & 0,000 & $-3,530$ & 0,073 & 0,000 & $(-3,437 ;-3,438)$ \\
& $\beta$ & 0,437 & 0,019 & 0,000 & 0,432 & 0,023 & 0,000 & $(0,468 ; 0,468)$ \\
& $\phi$ & 109,105 & 18,169 & 0,000 & 116,889 & 32,892 & 0,003 & $(64,788 ; 160,651)$ \\
\hline \multirow{6}{*}{$\ell_{\text {Nave }}$} & $\alpha$ & $-3,539$ & 0,077 & 0,000 & $-3,529$ & 0,072 & 0,000 & $(-3,651 ;-3,424)$ \\
& $\beta$ & 0,436 & 0,020 & 0,000 & 0,431 & 0,023 & 0,000 & $(0,401 ; 0,464)$ \\
& $\phi$ & 109,104 & 17,215 & 0,000 & 116,505 & 32,555 & 0,002 & $(65,076 ; 158,781)$ \\
\hline
\end{tabular}

mos observar que as estimativas e os erros padrões dos estimadores do parâmetro de precisão tiveram um ligeiro incremento quando este é estimado via máxima verossimilhança aproximada e pseudo-verossimilhança aproximada. Resultados semelhantes foram obtidos quando utilizamos o método bootstrap. Para dar continuidade à nossa análise utilizaremos apenas o método de estimação via máxima pseudo-verossimilhança aproximada, dado que este apresentou melhores resultados no comportamento dos estimadores (estudo de simulação) e é computacionalmente mais eficiente.

Com o intuito de verificar possíveis afastamentos das suposições feitas para o modelo construí- 
mos o gráfico do resíduos ponderados padronizados 2 dado na Seção 4.2.1 contra os índices das observações e contra os valores preditos da variável não observada $x_{t}$. Na Figura 5.1 apresentamos os gráficos dos resíduos ponderados padronizados 2 versus índice das observações (Figura 5.1(a)) e versus os valores preditos da variável não observada $x_{t}$ (Figura 5.1(b)). Observamos que os resíduos se distribuem aleatoriamente em torno de zero, as observações, $\sharp 5$ e $\sharp 60$ destacando-se como possíveis pontos aberrantes.

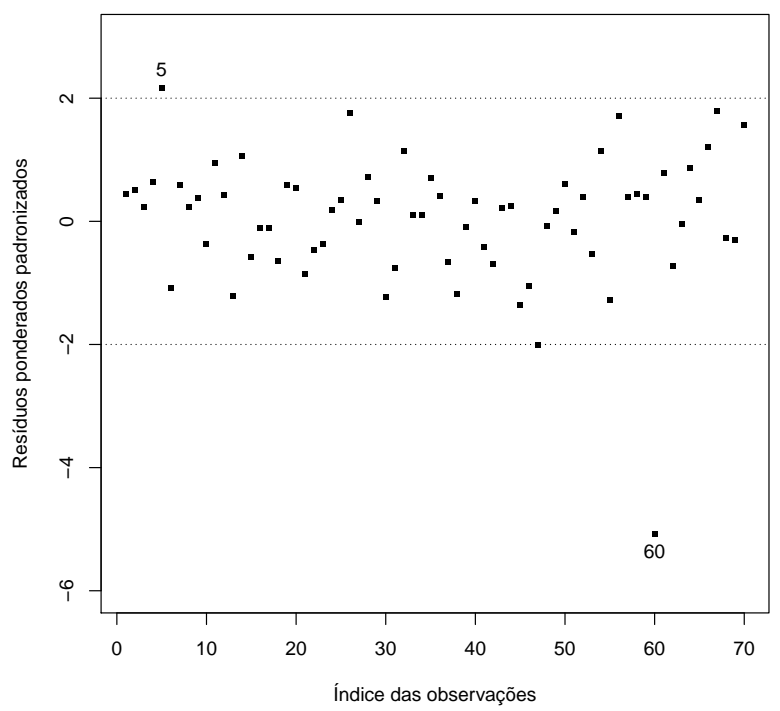

(a)

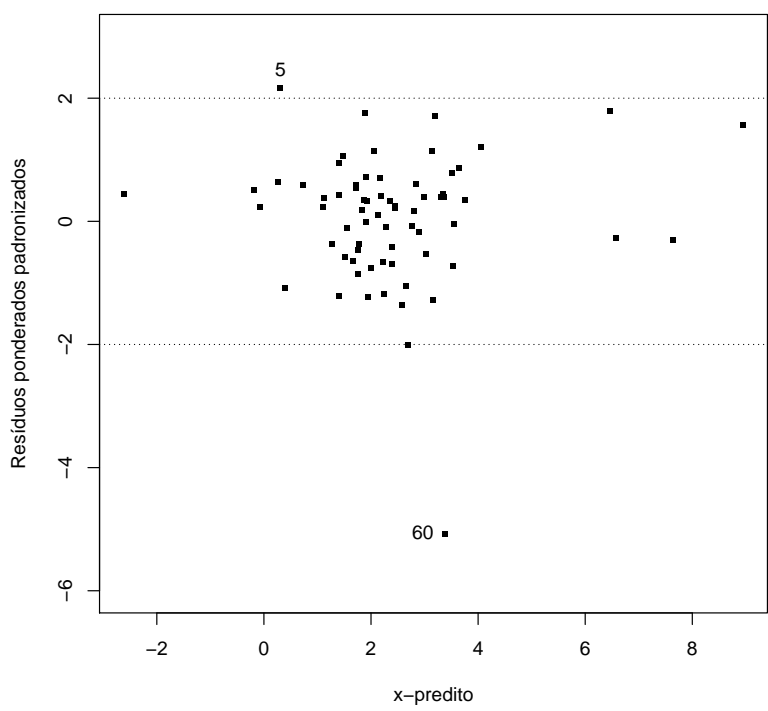

(b)

Figura 5.1: Gráfico do resíduo ponderado padronizado versus (a) indice das observações e (b) valores preditos de $x$.

Complementamos a análise de diagnóstico através de uma análise de influência com o objetivo de verificar a existência de possíveis pontos influentes nos dados. Aplicamos as técnicas de influência global e local vistas nas Seções 4.3 e 4.4, respectivamente. No que diz respeito à medida de influência local, utilizamos a proposta de Poon e Poon (1999) sob os esquemas de perturbação de casos, da resposta e da covariável medida com erro.

A Figura 5.2 mostra os gráficos das medidas de influência global, distância de Cook generalizada $C D_{t}(\mathbf{\Psi})$ e afastamento da verossimilhança $L D_{t}(\mathbf{\Psi})$, contra o índice das observações. Estes gráficos mostram a existência de possíveis pontos influentes: $\sharp 1, \sharp 5, \sharp 60, \sharp 67, \sharp 68, \sharp 69$ e $\sharp 70$. Continuando com a análise de influência, construímos gráficos de influência local conformal. Consideramos $q=1$ e 2 para determinar quais autovalores estão associados às variações máximas. Podemos observar nos gráficos de autovalores normalizados (Figuras 5.3(a) e 5.4(a)) que para $q=1$ há um único autovalor acima da linha de referência $q / \sqrt{n}$. No entanto, a Figura 5.5(a) mostra dois autovalores acima de $q / \sqrt{n}$. A máxima curvatura normal conformal, $B_{d \max }$, para os esquemas de perturbação de casos, da resposta e da covariável medida com erro são dados por 0,996, 0,998 e 0,983, respectivamente. As curvaturas normais conformais máximas podem ser interpretadas como a proporção do afastamento da verossimilhança que é explicada pelos autovetores q-influentes. As Figuras 5.3(b) e 5.3(c) mostram as contribuições agregadas de um autovetor $(q=1)$ e a contribuição agregada de todos os autovetores $(q=0)$. Como é sugerido por Poon e Poon (1999) determinamos o valor de corte $2 b$, 


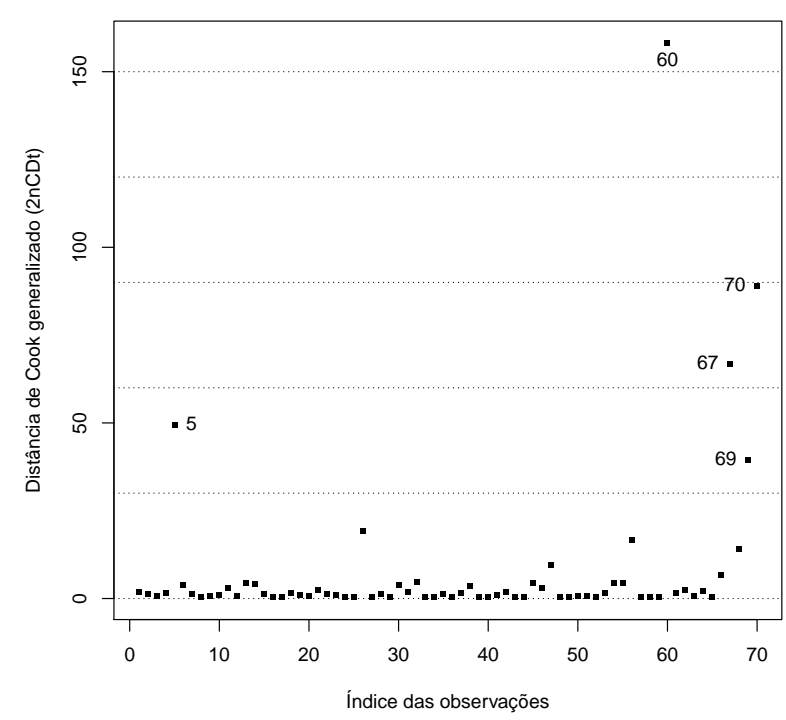

(a)

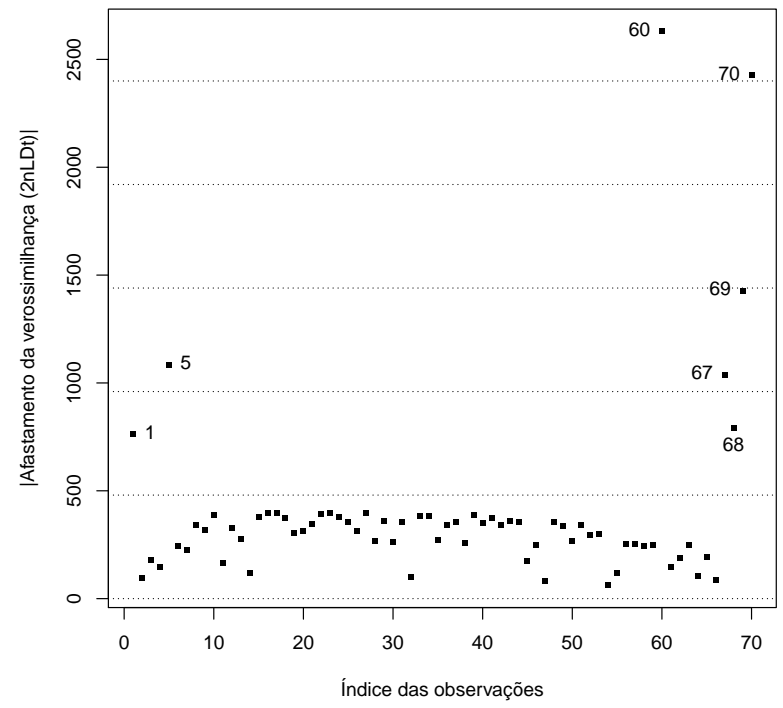

(b)

Figura 5.2: Medidas de influência global, (a) distância de Cook generalizado e (b) afastamento da verossimilhança.

ou seja as observações com medida maiores do que $2 b$ são consideradas influentes. Para o caso do esquema de perturbação de casos o valor de corte é $2 b=0,1037$. Acima do valor de corte existem muitas observações. Para nossa análise selecionamos aquelas que estão muito distantes, ou seja, as observações $\sharp 5, \sharp 60, \sharp 67$ e $\sharp 70$.

Para o caso dos esquemas de perturbação da resposta e da covariável temos como ponto de corte 0,097 e 0,153, respectivamente. As observações consideradas como possíveis pontos influentes foram $\sharp 5, \sharp 60, \sharp 67$ e $\sharp 70$.

Realizamos a retirada das observações detectadas como aberrantes na análise de resíduos e influentes nos esquemas de perturbação, uma a uma e conjuntamente, com a finalidade de verificar o impacto causado no ajuste do modelo. Utilizamos o desvio relativo percentual (DRP) para avaliar a magnitude do impacto exercido pelas observações. Para $\boldsymbol{\Psi}=\left(\Psi_{1}, \Psi_{2}, \Psi_{3}\right)^{\top}=(\alpha, \beta, \phi)^{\top}$, temos que o desvio relativo percentual é dado por

$$
D R P_{j}=\frac{\widehat{\mathbf{\Psi}}_{j}-\widehat{\mathbf{\Psi}}_{j}^{*}}{\widehat{\mathbf{\Psi}}_{j}} \times 100 \%
$$

em que $\widehat{\mathbf{\Psi}}_{j}^{*}$ é o estimador do parâmetro $\boldsymbol{\Psi}_{j}$ encontrado ao retirar uma ou várias observações atípicas para $j=1,2,3$. Na Tabela 5.5 apresentamos as estimativas, o erro padrão e o desvio relativo percentual das estimativas dos parâmetros ao retirar uma a uma e conjuntamente as observações consideradas como influentes e aberrantes. Notamos que as estimativas do parâmetro de precisão $\phi$ sofreram um forte impacto quando as observações $\sharp 5$ e $\sharp 60$ foram retiradas uma a uma e conjuntamente. Não ocorre o mesmo com o intercepto e o parâmetro associado à variável medida com erro. Desta maneira, podemos concluir que, entre as observações detectadas como aberrantes ou influentes, as que afetam alguns resultados inferenciais do modelo são $\sharp 5$ e $\sharp 60$.

Apresentamos a seguir os gráficos normais de probabilidades com envelope simulado para avaliar 


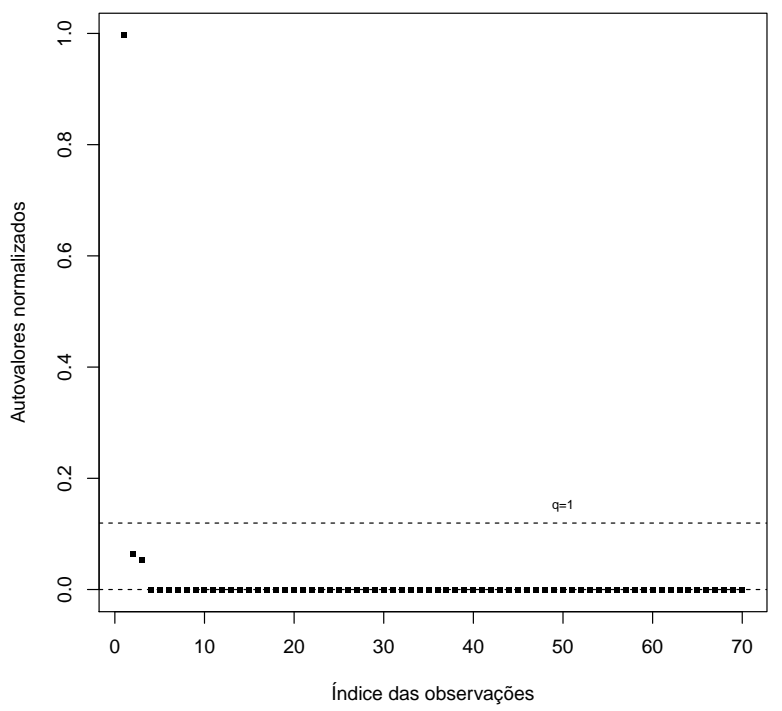

(a)

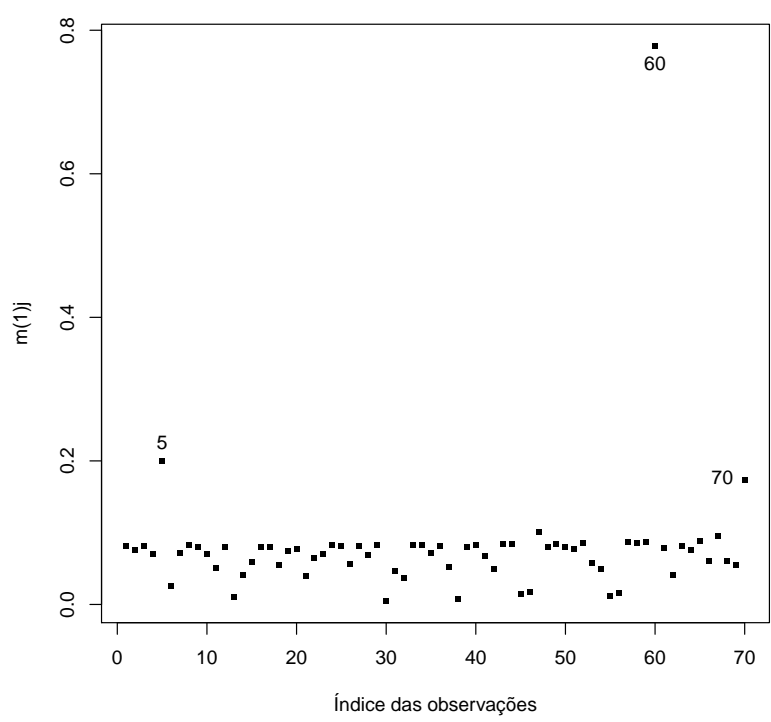

(b)

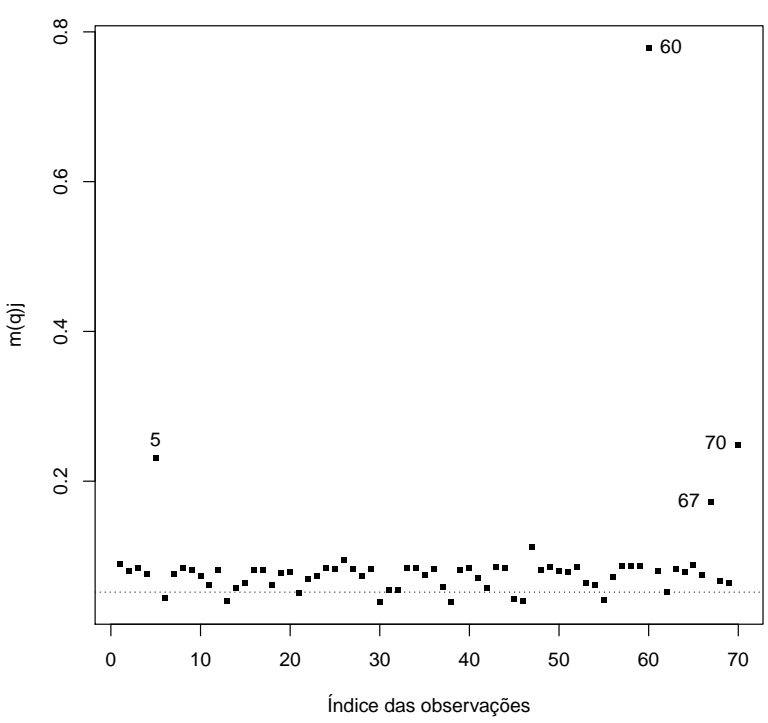

(c)

Figura 5.3: (a) Autovalores normalizados em módulo com valores de q, (b) influência devida à contribuição agregada do autovetor 1-influente e (c) influência devida à contribuição agregada de todos os autovetores, $m[q]_{j}$ para o esquema de perturbação de casos. 


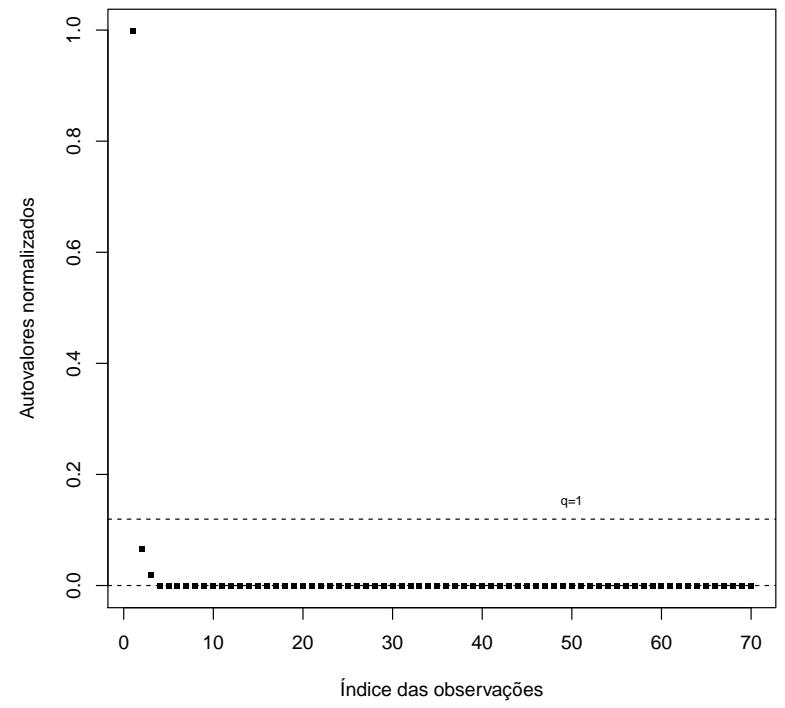

(a)

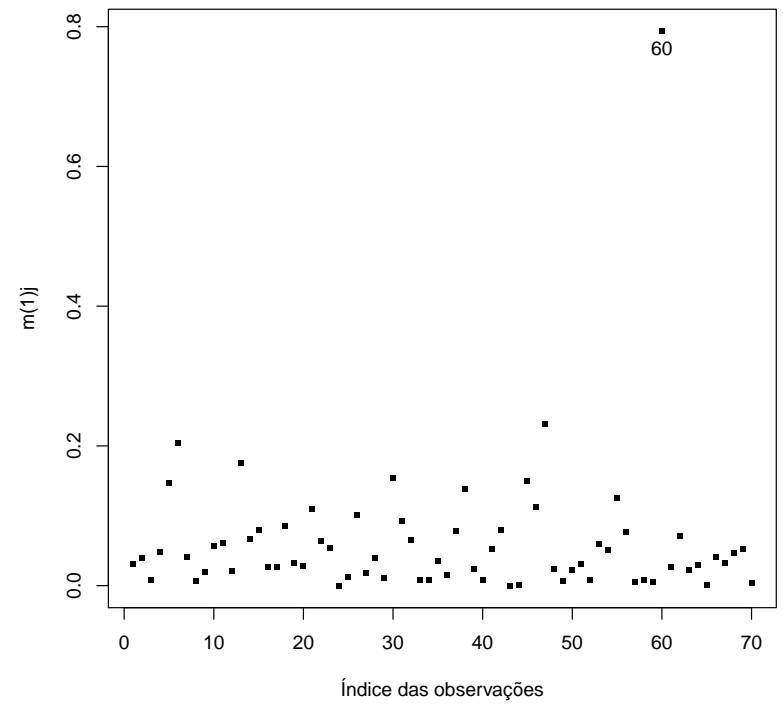

(b)

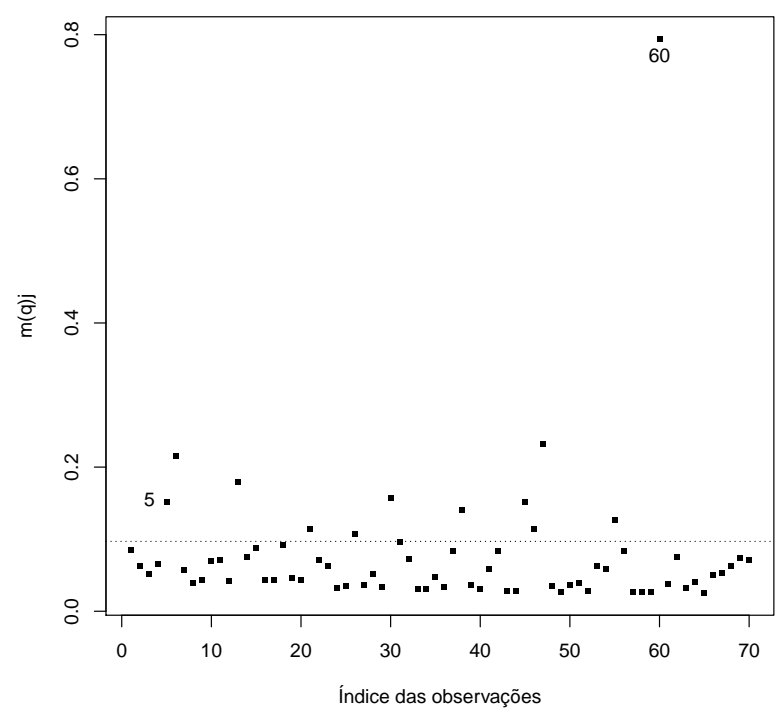

(c)

Figura 5.4: (a) Autovalores normalizados em módulo com valores de q, (b) influência devida à contribuição agregada do autovetor 1-influente e (c) influência devida à contribuição agregada de todos os autovetores, $m[q]_{j}$ para o esquema de perturbação da variável resposta. 


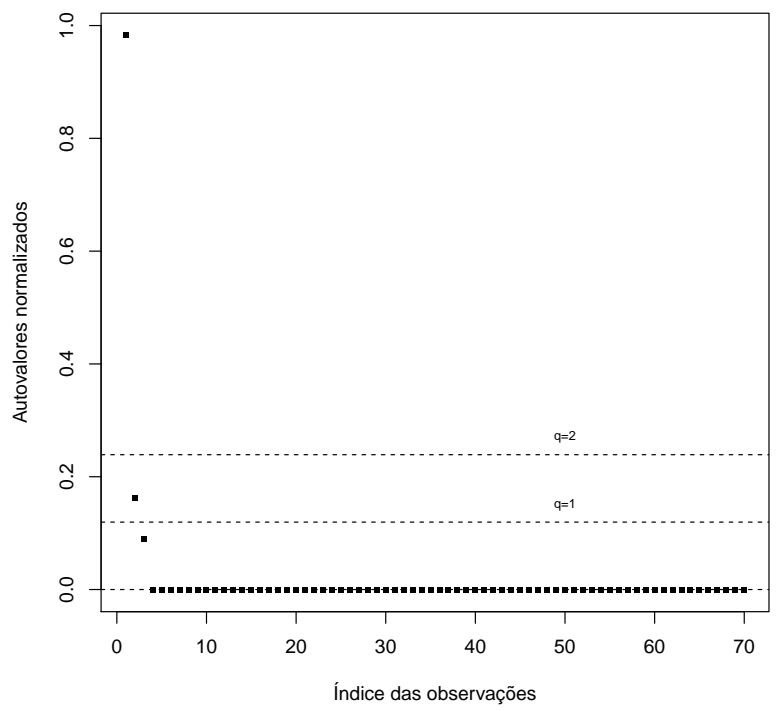

(a)

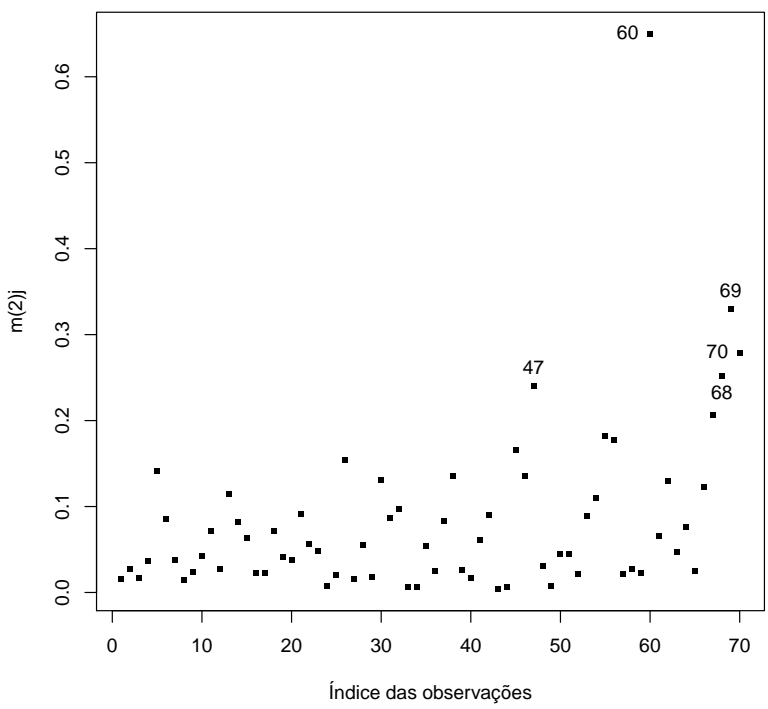

(b)

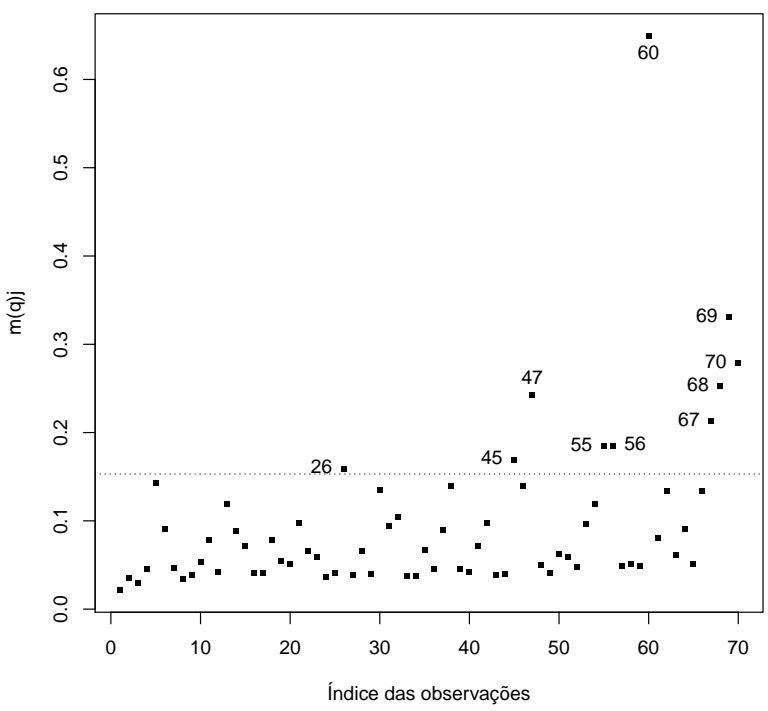

(c)

Figura 5.5: (a) Autovalores normalizados em módulo com valores de q, (b) influência devida à contribuição agregada do autovetor 2-influente e (c) influência devida à contribuição agregada de todos os autovetores, $m[q]_{j}$ para o esquema de perturbação da variável resposta. 
Tabela 5.5: Estimativas (E), erros padrão (EP) e desvio relativo percentual (DRP) obtidos pelo método de máxima pseudo-verossimilhança.

\begin{tabular}{c|c|rrr}
\hline Exclusão & $\widehat{\boldsymbol{\theta}}$ & \multicolumn{1}{|c}{$E$} & \multicolumn{1}{c}{$E P$} & $D R P(\%)$ \\
\hline \multirow{4}{*}{ Nenhuma } & $\alpha$ & $-3,545$ & 0,070 & - \\
& $\beta$ & 0,438 & 0,018 & - \\
& $\phi$ & 112,377 & 33,963 & - \\
\hline \multirow{3}{*}{$\sharp 5$} & $\alpha$ & $-3,581$ & 0,062 & 1,016 \\
& $\beta$ & 0,445 & 0,016 & 1,598 \\
& $\phi$ & 125,664 & 40,029 & 11,824 \\
\hline \multirow{4}{*}{$\sharp 60$} & $\alpha$ & $-3,542$ & 0,069 & $-0,085$ \\
& $\beta$ & 0,442 & 0,017 & 0,913 \\
$\sharp 67$ & $\phi$ & 153,026 & 31,095 & 36,172 \\
\hline \multirow{4}{*}{$\sharp 68$} & $\alpha$ & $-3,528$ & 0,072 & $-0,479$ \\
& $\beta$ & 0,428 & 0,018 & $-2,283$ \\
& $\phi$ & 114,497 & 34,815 & 1,887 \\
\hline \multirow{3}{*}{$\sharp 69$} & $\alpha$ & $-3,553$ & 0,072 & 0,226 \\
& $\beta$ & 0,442 & 0,019 & 0,913 \\
& $\phi$ & 111,526 & 34,139 & $-0,757$ \\
\hline \multirow{4}{*}{$\sharp 60$} & $\beta$ & $-3,564$ & 0,072 & 0,536 \\
& $\phi$ & 0,446 & 0,019 & 1,826 \\
& $\alpha$ & 112,108 & 34,579 & $-0,240$ \\
\hline 70 & $\alpha$ & $-3,512$ & 0,079 & $-0,931$ \\
& $\phi$ & 0,422 & 0,025 & $-3,653$ \\
& $\phi$ & 111,456 & 33,135 & $-0,820$ \\
\hline & $\alpha$ & $-3,577$ & 0,062 & 0,902 \\
& 0,449 & 0,016 & 2,511 \\
& 179,217 & 30,917 & 59,478 \\
\hline & & &
\end{tabular}

a adequabilidade do modelo de regressão beta com erros de medida para os dados. A Figura 5.6 mostra os gráficos de envelope simulado quando consideramos os dados completos, retirando a observação $\sharp 60$ como também retirando simultaneamente as observações $\sharp 5$ e $\sharp 60$. A Figura 5 .6(a) mostra que os resíduos ponderados padronizados localizam-se no interior dos envelopes simulados com exceção da observação $\sharp 60$. No entanto, as Figuras 5.6(b) e 5.6(c) mostram uma leve influência na falta de ajuste do modelo aos dados quando são retiradas as observações consideradas como influentes ou aberrantes. Concluímos que o modelo de regressão beta com erros de medida se ajusta bem ao conjunto de dados mesmo tendo como observação aberrante e influente os casos $\sharp 5$ e $\sharp 60$.

\subsection{Aplicação II}

Nesta seção analisamos o conjunto de dados referente à doença arterial coronária (aterosclerose) mencionado na Seção 1.1.2. Esta doença é diagnosticada pelo endurecimento dos vasos sanguíneos causado pelo depósito de placas de gordura. O colesterol é uma substância gordurosa que está presente em todas as células do nosso corpo. Ele trafega em nosso sangue através de partículas chamadas de lipoproteínas. Três das lipoproteínas comuns são: lipoproteínas de baixa densidade 


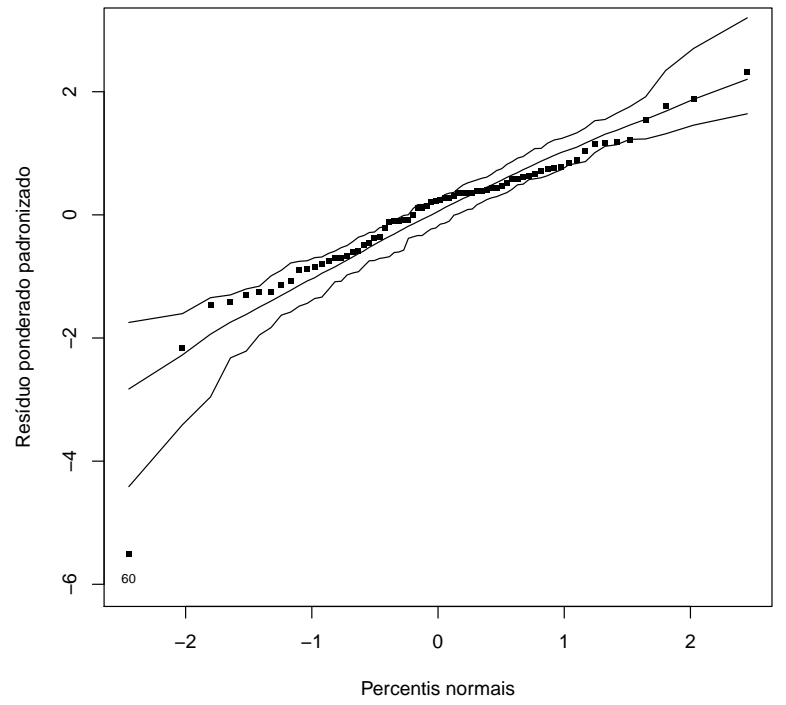

(a)

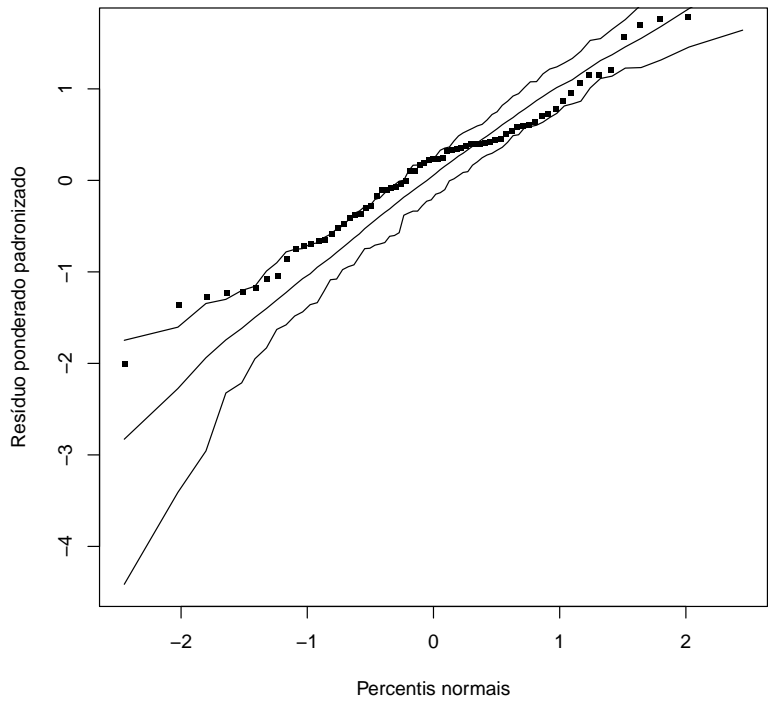

(b)

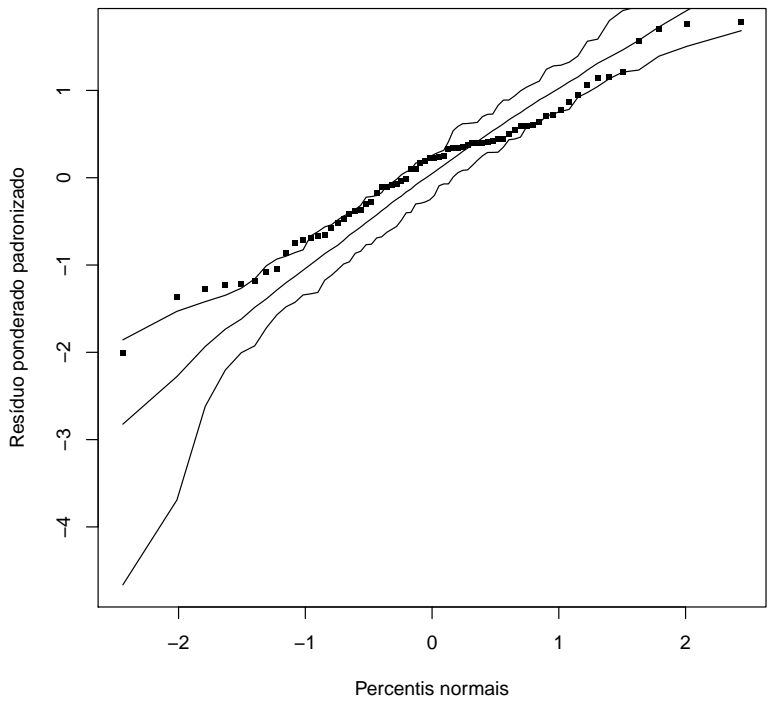

(c)

Figura 5.6: Gráfico normal de probabilidades com envelope simulado com (a) dados completos, (b) retirando a observação $\sharp 60$ e (c) retirando as observações $\sharp 5$ e $\sharp 60$. 
(LDL), lipoproteínas de alta densidade (HDL) e lipoproteínas de muita baixa densidade (VLDL). Estudos médicos têm mostrado que níveis elevados de colesterol LDL estão associados ao desenvolvimento de placas nas artérias coronárias, os vasos que irrigam o coração, enquanto níveis elevados de colesterol HDL reduzem esse risco. Assim, muitos referem-se ao LDL como o colesterol ruim e ao HDL como o colesterol bom. A forma mais comum de medir o colesterol de um indivíduo é medindo o colesterol total presente no sangue. O nível de colesterol total poderá ser verificado através de uma pequena amostra de sangue colhida no dedo ou no braço. Se o colesterol total for igual ou superior a $190 \mathrm{mg} / \mathrm{dl}$ (miligramas por decilitro) será conveniente coletar uma amostra sanguínea em jejum. A análise dessa amostra é feita após um período de 10 a 12 horas, dando as seguintes informações: colesterol total (CT), colesterol HDL, colesterol LDL e trigliceridos. O colesterol total é constituído do colesterol LDL, colesterol HDL e colesterol VLDL, ou seja, CT=LDL + HDL + VLDL.

A American Heart Association ${ }^{1}$ relaciona os seguintes níveis de colesterol total ao risco de doenças cardíacas: níveis desejados com menor risco de doença cardíaca quando $\mathrm{CT}<200 \mathrm{mg} / \mathrm{dl}$, e considera-se de alto risco quando CT $>240 \mathrm{mg} / \mathrm{dl}$. No entanto, o nível desejável de colesterol LDL é menor do que $100 \mathrm{mg} / \mathrm{dl}$.

Pessoas fumantes possuem chances maiores de desenvolver aterosclerose coronária do que pessoas não fumantes; o risco se eleva com a profundidade da inalação do fumo e com o número total de anos em que fumou, pois o ato de fumar aumenta a adesividade das plaquetas, além de contribuir com o aumento da pressão arterial. Níveis elevados da pressão arterial (hipertensão arterial) são indicadores de risco cardíaco. Há duas medidas que caracterizam a pressão arterial: pressão arterial sistólica e diastólica. Valores de $14 \mathrm{mmHg}$ para a pressão sistólica e $9 \mathrm{mmHg}$ para a diastólica podem ser aceitas como normais. A pressão do pulso (PP), que é a diferença entre as pressões arterial sistólica e diastólica, é também uma medida que indica problemas cardíacos. Segundo o American College of Surgeons (2008), a pressão de pulso é considerada anormalmente baixa se for inferior ao $25 \%$ do valor sistólico, sendo a causa a insuficiência cardíaca congênita.

Por outro lado, pesquisas médicas mostram que o excesso de peso está intimamente associado aos fatores de risco cardíaco, pois eleva a pressão arterial. Medidas como o índice de massa corporal (IMC) caracterizam as pessoas segundo seu peso e altura. Segundo a Organização Mundial da Saúde ${ }^{2}$ (OMS) uma pessoa com IMC $<18,5 \mathrm{~kg} / \mathrm{m}^{2}$ apresenta baixo peso, se 18,6 $<\mathrm{IMC}<24,9$ é saudável, se $25<\mathrm{IMC}<29,9$ encontra-se com sobrepeso, se $30<\mathrm{IMC}<34,9$ considera-se o indivíduo com obesidade grau I, se $35<\mathrm{IMC}<39,9$ com obesidade grau II e se o IMC $>40 \mathrm{~kg} / \mathrm{m}^{2}$ com obesidade grau III.

Consideramos aqui o estudo desenvolvido pelo Departamento de Biometria da Universidade Estadual Paulista "Júlio de Mesquita Filho" que objetiva encontrar alguns fatores de risco cardíaco em 182 mulheres fumantes com idades entre 50 e 87 anos, tal como foi mostrado na Seção 1.1.2. Nosso objetivo, é explicar o comportamento de $y=(P A S-P A D) / P A S=P P / P A S$ usando as variáveis IMC e LDL. Assumiremos nesta aplicação que as variáveis $y$, IMC e LDL encontram-se livres de erros de medição. Como comentado acima a variável LDL é uma medida obtida sob algumas restrições, por exemplo, a coleta da amostra sanguínea tem que ser feita em jejum e os resultados da análise ficam prontos após 10 a 12 horas, além disso é requerido equipamentos especializados. Como o colesterol LDL é uma parcela do colesterol total (CT), utilizamos a variável CT (variável rápida e fácil de ser obtida) como uma variável substituta de LDL para ilustrar uma aplicação dos

\footnotetext{
${ }^{1}$ http://www.heart.org

${ }^{2}$ http://www.who.int/
} 
resultados obtidos nesta tese.

Aqui, a variável de interesse é contínua em $(0,1)$ e a covariável CT é considerada como uma variável substituta de LDL. É portanto, adequado considerar um modelo de regressão beta com erros de medida. Note que o erro de medição de CT não é de interesse primário; mas sim, das quantidades desconhecidas dos outros componentes (HDL e VLDL) que produzem erro de medição (Roeder et al., 1996). Consideramos as transformações seguintes LDL/100 e TC/100 para $x$ e $w$.

Realizamos uma análise descritiva do conjunto de dados. Na Tabela 5.6 observa-se que a variável resposta pertence ao intervalo $[0,208 ; 0,588]$ e que, em média, as mulheres fumantes apresentam sobrepeso. Considerando os níveis de colesterol segundo a American Heart Association, mais da metade das mulheres fumantes da amostra encontram-se em risco cardíaco, uma vez que, os níveis medianos de colesterol total e ruim são 214,5 e $130,6 \mathrm{mg} / \mathrm{dl}$, respectivamente.

Tabela 5.6: Medidas resumo das variáveis

\begin{tabular}{c|rrrrrrr}
\hline Variáveis & Mínimo & \multicolumn{1}{c}{$Q_{1}$} & Mediana & Média & \multicolumn{1}{c}{$Q_{3}$} & Máximo & Desvio padrão \\
\hline$y$ & 0,208 & 0,348 & 0,375 & 0,385 & 0,416 & 0,588 & 0,059 \\
$I M C$ & 18,189 & 25,206 & 27,680 & 28,206 & 30,559 & 44,266 & 4,423 \\
$C T$ & 1,070 & 1,893 & 2,145 & 2,142 & 2,330 & 3,460 & 0,394 \\
$L D L$ & 0,436 & 1,105 & 1,306 & 1,323 & 1,508 & 2,594 & 0,357 \\
\hline
\end{tabular}

A Figura 5.7 mostra a existência de uma relação linear entre o colesterol total e LDL. Essa característica é útil para a escolha do modelo com erros de medida dada em (2.6).

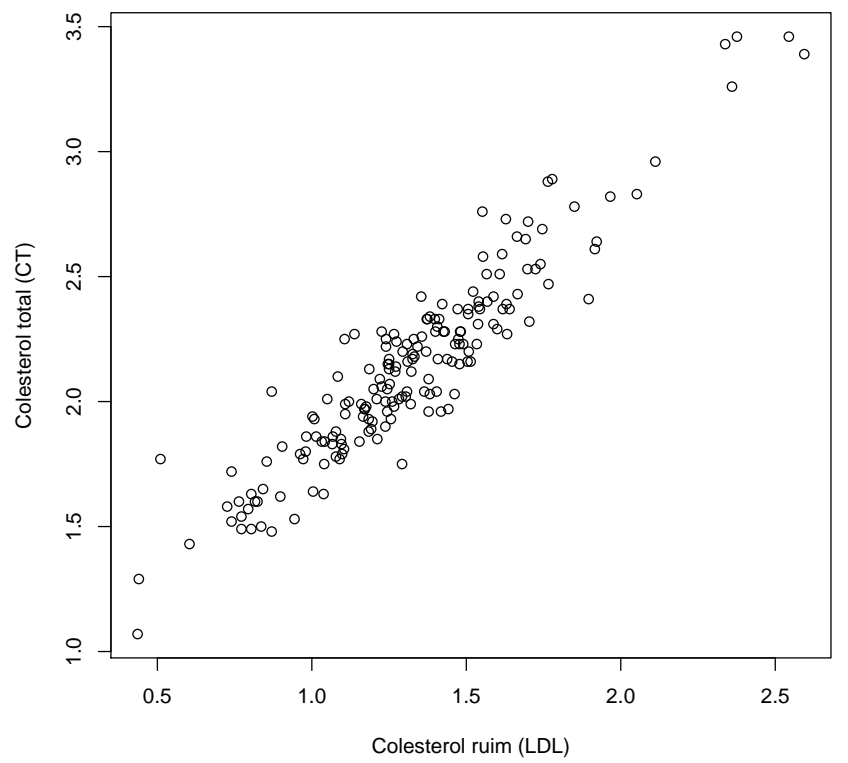

Figura 5.7: Gráfico de dispersão do colesterol total (CT) e colesterol ruim (LDL) ambas medições em $m g / d l$.

Admitimos agora que $y_{1}, y_{2}, \ldots, y_{n}$ são variáveis aleatórias independentes tais que $y_{t}$, para todo 
$t=1, \ldots, n$, tem distribuição beta de média $\mu_{t}$ e parâmetro de precisão $\phi_{t}$. Ademais,

$$
\begin{aligned}
\log \left(\frac{\mu_{t}}{1-\mu_{t}}\right) & =\alpha_{0}+\alpha_{1} \mathrm{IMC}_{t}+\beta \mathrm{LDL}_{t} \\
\log \left(\phi_{t}\right) & =\gamma_{0}+\gamma_{1} \mathrm{IMC}_{t}+\lambda \mathrm{LDL}_{t} \\
\mathrm{CT}_{t} & =\tau_{0}+\tau_{1} \mathrm{LDL}_{t}+e_{t}
\end{aligned}
$$

em que $t=1, \ldots, n, L D L_{t} \sim N\left(\mu_{x}, \sigma_{x}^{2}\right)$ e $e_{t} \sim N\left(0, \sigma_{e}^{2}\right)$. Aqui, o vetor de parâmetros de interesse é $\boldsymbol{\theta}=\left(\alpha_{0}, \alpha_{1}, \beta, \gamma_{0}, \gamma_{1}, \lambda\right)^{\top}$ e o vetor de parâmetros de perturbação é $\boldsymbol{\xi}=\left(\tau_{0}, \tau_{1}, \mu_{x}, \sigma_{x}^{2}, \sigma_{e}^{2}\right)^{\top}$. É possível determinar as estimativas de $\tau_{0}, \tau_{1}$ e $\sigma_{e}^{2}$ a partir do modelo (5.2), dado que nosso conjunto de dados contém as medições do colesterol total e ruim para todos os indivíduos. As estimativas dos parâmetros e respectivos erros padrão são: $\widehat{\tau}_{0}=0,776(0,039), \widehat{\tau}_{1}=1,033(0,028)$ e $\widehat{\sigma}_{e}^{2}=0,019$. As variáveis $C T$ e $L D L$ apresentam uma correlação de 0.937 . As estimativas, erros padrão e $p$-valor dos parâmetros obtidos a partir dos métodos de máxima verossimilhança aproximada, pseudoverossimilhança aproximada, calibração da regressão e naïve são apresentados na Tabela 5.7. Nesta tabela, determinamos também resultados inferenciais utilizando técnicas de reamostragem bootstrap não paramétrico. Para comparação, utilizamos o modelo considerado sem erros, isto é, quando a covariável medida com erro é o próprio LDL.

Da Tabela 5.7 concluímos que os métodos de estimação utilizando $\ell_{a}$ e $\ell_{p}$ apresentam resultados mais próximos daqueles do modelo sem erros. Consideramos o modelo sem erros quando utilizamos diretamente a variável $L D L$. Observamos também que os resultados obtidos quando utilizamos $\ell_{R C}$ e $\ell_{\text {Naive }}$ apresentam incoerências frente ao modelo sem erros, por exemplo, em ambos casos existem indícios para não rejeitar a hipótese $\mathcal{H}_{0}: \lambda=0$ a um nível de significância de $5 \%$ ao contrário do que ocorre no modelo sem erros e quando os parâmetros são estimados via máxima verossimilhança aproximada ou máxima pseudo-verossimilhança aproximada. Consideramos agora, o teste das hipóteses $\mathcal{H}_{0}: \gamma_{1}=0$ e $\mathcal{H}_{1}: \gamma_{1} \neq 0$ para verificar a significância ou não da variável IMC quando é considerada no modelo de precisão. A Tabela 5.8 mostra os valores das estatísticas da razão de verossimilhanças e gradiente com seus respectivos p-valores entre parênteses. Dos resultados da tabela, concluímos que não existem evidências suficientes para rejeitar $\mathcal{H}_{0}$ com os níveis de significância usuais. Consideramos agora o modelo sem a variável IMC associada ao modelo de precisão. Obtemos as estimativas, erros padrão e $p$-valores para o conjunto de dados, como mostra a Tabela 5.9.

Dos resultados apresentados na Tabela 5.9 concluímos que os métodos de regressão da calibração e naïve continuam apresentando diferenças significativas com respeito ao modelo sem erros. No entanto, as estimativas dos parâmetros e erros padrão aproximam-se mais do modelo sem erros para $\ell_{a}$ e $\ell_{p}$. Utilizaremos o método de máxima pseudo-verossimilhança para as próximas análises, pois este apresenta menor esforço computacional.

Verificamos a existência de possíveis afastamentos das suposições inerentes ao modelo, construindo gráficos do resíduos ponderados padronizados, tal como mostra a Figura 5.8. O gráfico da Figura 5.8(a) é construído a partir dos resíduos ponderados padronizados versus o índice das observações e o gráfico 5.8(b) é construído a partir dos resíduos ponderados padronizados versus valores preditos da variável $L D L_{t}$. Observa-se nesses gráficos a existência de evidências fortes para afirmar que o resíduo proposto se distribui aleatoriamente em torno de zero no intervalo [-2;2]. Ademais, existe a presença de possíveis quatro pontos aberrantes, $\sharp 31, \sharp 74, \sharp 131$ e $\sharp 163$. Do conjunto de dados 
Tabela 5.7: Estimativas (E), erros padrão (EP), valor-p (p) e intervalo de confiança (IC) obtidos pelos métodos de máxima verossimilhança, pseudo-verossimilhança e bootstrap.

\begin{tabular}{|c|c|c|c|c|c|c|c|c|}
\hline & \multirow{2}{*}{\multicolumn{4}{|c|}{ bootstrap }} \\
\hline & & & & & & & & \\
\hline Método & $\widehat{\theta}$ & $E$ & $E P$ & $p$ & $E$ & $E P$ & $p$ & $I C$ \\
\hline \multirow{6}{*}{ Sem erros } & $\alpha_{0}$ & $-0,354$ & 0,124 & 0,004 & $-0,369$ & 0,130 & 0,002 & $(-0,535 ;-0,117)$ \\
\hline & $\alpha_{1}$ & $-0,009$ & 0,004 & 0,023 & $-0,009$ & 0,004 & 0,014 & $(-0,016 ;-0,005)$ \\
\hline & $\beta$ & 0,107 & 0,050 & 0,034 & 0,107 & 0,060 & 0,046 & $(0,010 ; 0,206)$ \\
\hline & $\gamma_{0}$ & 5,905 & 0,764 & 0,000 & 5,840 & 1,065 & 0,000 & $(4,247 ; 7,857)$ \\
\hline & $\gamma_{1}$ & $-0,022$ & 0,024 & 0,356 & $-0,018$ & 0,038 & 0,268 & $(-0,092 ; 0,036)$ \\
\hline & $\lambda$ & $-0,729$ & 0,292 & 0,013 & $-0,734$ & 0,279 & 0,010 & $(-1,145 ;-0,245)$ \\
\hline \multirow{6}{*}{$\ell_{a}$} & $\alpha_{0}$ & $-0,344$ & 0,129 & 0,008 & $-0,358$ & 0,129 & 0,004 & $(-0,541 ;-0,107)$ \\
\hline & $\alpha_{1}$ & $-0,009$ & 0,004 & 0,024 & $-0,009$ & 0,004 & 0,018 & $(-0,017 ;-0,004)$ \\
\hline & $\beta$ & 0,103 & 0,055 & 0,064 & 0,101 & 0,064 & 0,056 & $(0,000 ; 0,209)$ \\
\hline & $\gamma_{0}$ & 5,951 & 0,879 & 0,000 & 5,812 & 1,148 & 0,000 & $(4,209 ; 8,107)$ \\
\hline & $\gamma_{1}$ & $-0,028$ & 0,028 & 0,310 & $-0,021$ & 0,040 & 0,249 & $(-0,098 ; 0,037)$ \\
\hline & $\lambda$ & $-0,628$ & 0,290 & 0,030 & $-0,637$ & 0,354 & 0,043 & $(-1,173 ;-0,041)$ \\
\hline \multirow{6}{*}{$\ell_{p}$} & $\alpha_{0}$ & $-0,344$ & 0,125 & 0,006 & -0.358 & 0,130 & 0,005 & $(-0.541 ;-0.107)$ \\
\hline & $\alpha_{1}$ & $-0,009$ & 0,004 & 0,013 & -0.009 & 0,004 & 0,018 & $(-0.017 ;-0.004)$ \\
\hline & $\beta$ & 0,103 & 0,060 & 0,088 & 0.101 & 0,065 & 0,056 & $(0.000 ; 0.209)$ \\
\hline & $\gamma_{0}$ & 5,931 & 0,970 & 0,000 & 5.812 & 1,095 & 0,000 & $(4.209 ; 8,107)$ \\
\hline & $\gamma_{1}$ & $-0,028$ & 0,036 & 0,435 & -0.021 & 0,039 & 0,249 & $(-0,098 ; 0,037)$ \\
\hline & $\lambda$ & $-0,628$ & 0,283 & 0,026 & -0.637 & 0,351 & 0,043 & $(-1.173 ;-0.041)$ \\
\hline \multirow{6}{*}{$\ell_{R C}$} & $\alpha_{0}$ & $-0,340$ & 0,129 & 0,009 & $-0,356$ & 0,128 & 0,004 & $(-0,539 ;-0,097)$ \\
\hline & $\alpha_{1}$ & $-0,009$ & 0,004 & 0,023 & $-0,009$ & 0,004 & 0,018 & $(-0,017 ;-0,005)$ \\
\hline & $\beta$ & 0,100 & 0,055 & 0,068 & 0,098 & 0,064 & 0,064 & $(-0,001 ; 0,210)$ \\
\hline & $\gamma_{0}$ & 5,849 & 0,776 & 0,000 & 5,732 & 1,057 & 0,000 & $(4,293 ; 7,935)$ \\
\hline & $\gamma_{1}$ & $-0,028$ & 0,024 & 0,239 & $-0,023$ & 0,039 & 0,244 & $(-0,097 ; 0,033)$ \\
\hline & $\lambda$ & $-0,577$ & 0,312 & 0,064 & $-0,568$ & 0,321 & 0,056 & $(-1,047 ; 0,001)$ \\
\hline \multirow{6}{*}{$\ell_{\text {Naive }}$} & $\alpha_{0}$ & $-0,389$ & 0,144 & 0,007 & $-0,405$ & 0,149 & 0,002 & $(-0,635 ;-0,139)$ \\
\hline & $\alpha_{1}$ & $-0,009$ & 0,004 & 0,023 & 0,009 & 0,004 & 0,018 & $(-0,017 ;-0,005)$ \\
\hline & $\beta$ & 0,085 & 0,047 & 0,068 & 0,083 & 0,055 & 0,064 & $(-0,001 ; 0,179)$ \\
\hline & $\gamma_{0}$ & 6,136 & 0,864 & 0,000 & 6,015 & 1,104 & 0,000 & $(4,585 ; 8,257)$ \\
\hline & $\gamma_{1}$ & $-0,028$ & 0,024 & 0,239 & $-0,023$ & 0,039 & 0,244 & $(-0,097 ; 0,033)$ \\
\hline & $\lambda$ & $-0,490$ & 0,265 & 0,064 & $-0,483$ & 0,273 & 0,056 & $(-0,890 ; 0,001)$ \\
\hline
\end{tabular}

temos que a observação $\sharp 31$ corresponde a uma mulher com pressão de pulso igual a $25 \mathrm{mmHg}$ $\left(y_{31}=0,208\right)$, IMC igual a $36,736 \mathrm{~kg} / \mathrm{m}^{2} \mathrm{com}$ CT e LDL iguais a 175 e $129,2 \mathrm{mg} / \mathrm{dl}$, respectivamente. O indivíduo correspondente ao caso $\sharp 74$ apresenta um PP igual a $30 \mathrm{mmHg}\left(y_{74}=0,214\right)$, IMC igual a $31,11 \mathrm{~kg} / \mathrm{m}^{2}$ e $223,146,4 \mathrm{mg} / \mathrm{dl}$ de CT e LDL, respectivamente. Em ambos os casos, a pressão de pulso é inferior a $25 \%$ da pressão arterial sistólica. Temos também a presença do caso $\sharp 163$ que apresenta PP igual a $85 \mathrm{mmHg}\left(y_{163}=0,531\right)$, IMC igual a $27,827 \mathrm{Kg} / \mathrm{m}^{2}, 179$ e 109,8 $m g / d l$ de CT e LDL, respectivamente. Esta mulher fumante que tem 70 anos apresenta um sobrepeso considerável. Finalmente, a observação $\sharp 163$ representa uma mulher fumante com a maior PP, 
Tabela 5.8: Testes de $\mathcal{H}_{0}: \gamma_{1}=0$ nas estatísticas da razão de verossimilhanças e gradiente, em parênteses os p-valores.

\begin{tabular}{c|rrrr}
\hline Teste & $\ell_{a}$ & $\ell_{p}$ & $\ell_{R C}$ & $\ell_{\text {Naive }}$ \\
\hline Razão de verossimilhanças & 1,062 & 0,916 & 1,042 & 1,042 \\
& $(0,303)$ & $(0,969)$ & $(0,922)$ & $(0,314)$ \\
Gradiente & 1,051 & 0,919 & 1,052 & 1,053 \\
& $(0,305)$ & $(0.968)$ & $(0,911)$ & $(0,304)$ \\
\hline
\end{tabular}

Tabela 5.9: Estimativas (E), erros padrão (EP), p-valores (p) e intervalo de confiança (IC) obtidos pelos métodos de máxima verossimilhança, pseudo-verossimilhança e bootstrap.

\begin{tabular}{|c|c|c|c|c|c|c|c|c|}
\hline \multirow[b]{2}{*}{ Método } & \multirow[b]{2}{*}{$\theta$} & \multirow[b]{2}{*}{$E$} & \multirow[b]{2}{*}{$E P$} & \multirow[b]{2}{*}{$p$} & \multicolumn{4}{|c|}{ bootstrap } \\
\hline & & & & & $E$ & $E P$ & $p$ & $I C$ \\
\hline \multirow{5}{*}{ Sem erros } & $\alpha_{0}$ & $-0,343$ & 0,124 & 0,005 & $-0,369$ & 0,130 & 0,002 & $(-0,535 ;-0,117)$ \\
\hline & $\alpha_{1}$ & $-0,009$ & 0,004 & 0,015 & $-0,009$ & 0,004 & 0,014 & $(-0,016 ;-0,005)$ \\
\hline & $\beta$ & 0,106 & 0,051 & 0,039 & 0,107 & 0,060 & 0,046 & $(0,010 ; 0,206)$ \\
\hline & $\gamma_{0}$ & 5,318 & 0,400 & 0,000 & 5,840 & 1,065 & 0,000 & $(4,247 ; 7,857)$ \\
\hline & $\lambda$ & $-0,752$ & 0,292 & 0,010 & $-0,734$ & 0,279 & 0,010 & $(-1,145 ;-0,245)$ \\
\hline \multirow{5}{*}{$\ell_{a}$} & $\alpha_{0}$ & $-0,330$ & 0,128 & 0,010 & $-0,337$ & 0,119 & 0,003 & $(-0,514 ;-0,106)$ \\
\hline & $\alpha_{1}$ & $-0,010$ & 0,004 & 0,013 & $-0,009$ & 0,004 & 0,010 & $(-0,017 ;-0,005)$ \\
\hline & $\beta$ & 0,102 & 0,056 & 0,068 & 0,101 & 0,063 & 0,060 & $(0,000 ; 0,209)$ \\
\hline & $\gamma_{0}$ & 5,127 & 0,408 & 0,000 & 5,138 & 0,517 & 0,000 & $(4,159 ; 5,868)$ \\
\hline & $\lambda$ & $-0,620$ & 0,294 & 0,035 & $-0,594$ & 0,361 & 0,054 & $(-1,157 ;-0,013)$ \\
\hline \multirow{5}{*}{$\ell_{p}$} & $\alpha_{0}$ & $-0,330$ & 0,119 & 0,005 & -0.337 & 0,119 & 0,003 & $(-0.514 ;-0.107)$ \\
\hline & $\alpha_{1}$ & $-0,010$ & 0,004 & 0,008 & -0.009 & 0,004 & 0,010 & $(-0.017 ;-0.005)$ \\
\hline & $\beta$ & 0,102 & 0,060 & 0,091 & 0.101 & 0,063 & 0,060 & $(0.000 ; 0.209)$ \\
\hline & $\gamma_{0}$ & 5,127 & 0,449 & 0,000 & 5.137 & 0,517 & 0,000 & $(4.159 ; 5,868)$ \\
\hline & $\lambda$ & $-0,620$ & 0,305 & 0,042 & -0.594 & 0,360 & 0,050 & $(-1.157 ;-0.017)$ \\
\hline \multirow{5}{*}{$\ell_{R C}$} & $\alpha_{0}$ & $-0,327$ & 0,130 & 0,012 & $-0,333$ & 0,117 & 0,003 & $(-0,509 ;-0,105)$ \\
\hline & $\alpha_{1}$ & $-0,010$ & 0,004 & 0,014 & $-0,010$ & 0,004 & 0,012 & $(-0,017 ;-0,005)$ \\
\hline & $\beta$ & 0,100 & 0,057 & 0,076 & 0,098 & 0,063 & 0,066 & $(0,000 ; 0,207)$ \\
\hline & $\gamma_{0}$ & 5,039 & 0,733 & 0,000 & 5,046 & 0,480 & 0,000 & $(4,158 ; 5,735)$ \\
\hline & $\lambda$ & $-0,562$ & 0,317 & 0,076 & $-0,535$ & 0,334 & 0,059 & $(-1,080 ; 0,013)$ \\
\hline \multirow{5}{*}{$\ell_{\text {Naive }}$} & $\alpha_{0}$ & $-0,377$ & 0,144 & 0,009 & $-0,381$ & 0,136 & 0,000 & $(-0,592 ;-0,131)$ \\
\hline & $\alpha_{1}$ & $-0,010$ & 0,004 & 0,014 & 0,010 & 0,004 & 0,012 & $(-0,017 ;-0,005)$ \\
\hline & $\beta$ & 0,085 & 0,047 & 0,070 & 0,083 & 0,054 & 0,066 & $(-0,001 ; 0,177)$ \\
\hline & $\gamma_{0}$ & 5,320 & 0,577 & 0,000 & 5,310 & 0,641 & 0,000 & $(4,195 ; 6,322)$ \\
\hline & $\lambda$ & $-0,477$ & 0,265 & 0,071 & $-0,454$ & 0,283 & 0,060 & $(-0,918 ; 0,014)$ \\
\hline
\end{tabular}

sendo $y_{131}=0,588$, com IMC $31,74 \mathrm{~kg} / \mathrm{m}^{2}, 203$ e 146,2 $\mathrm{mg} / \mathrm{dl}$ de CT e LDL, respectivamente.

Continuamos com nossa análise e construímos gráficos de influência global: distância de Cook generalizada e afastamento da verossimilhança, como mostra a Figura 5.9. Além das observações detectadas como aberrantes, a Figura 5.9 mostra a existência de mais pontos potencialmente influ- 


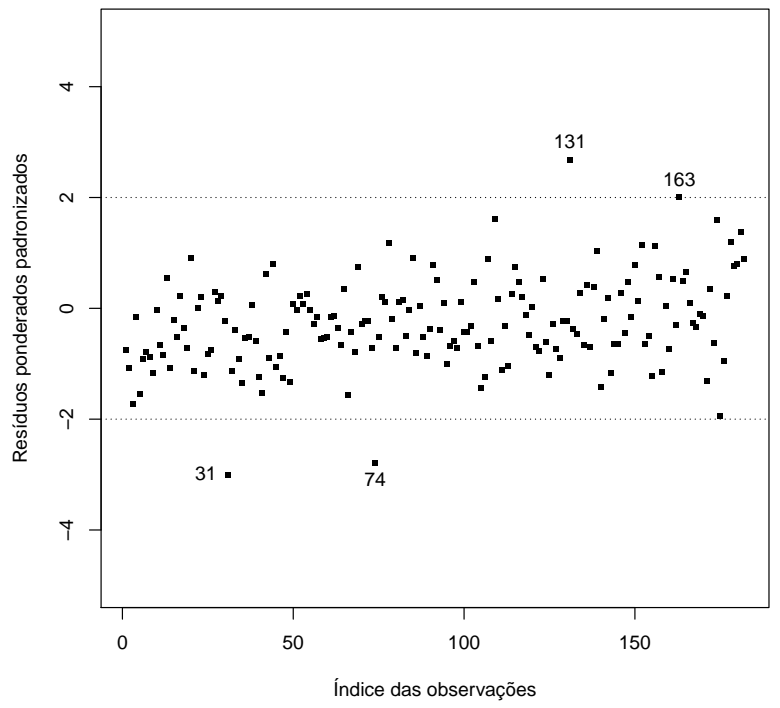

(a)

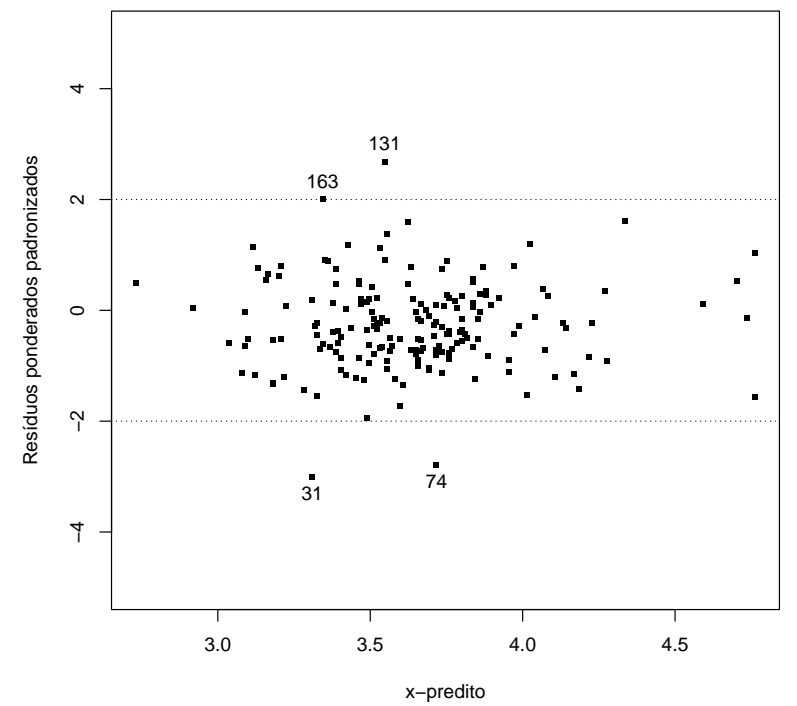

(b)

Figura 5.8: Gráfico do resíduo ponderado padronizado versus (a) indice das observações e (b) valores estimados de $x$.

entes, $\sharp 62, \sharp 66, \sharp 99, \sharp 109, \sharp 123$ e $\sharp 139$. Para verificar se os pontos detectados são influentes, tal como propõe Poon e Poon (1999), construímos gráficos de influência local conformal considerando valores $q=1,2,3,4,5,6$ para determinar quais autovalores estão associados às variações máximas Consideramos três esquemas de perturbação: ponderação de casos como mostra a Figura 5.10, perturbação da resposta, Figura 5.11 e perturbação da variável IMC, dado na Figura 5.12.

As Figuras 5.10, 5.11 e 5.12 detectaram como possíveis pontos influentes os casos $\sharp 5, \sharp 24, \sharp 31$, $\sharp 66, \sharp 74, \sharp 109, \sharp 131, \sharp 140, \sharp 163$ e $\sharp 175$. Para os esquemas de perturbação de casos e resposta, podemos observar nos gráficos de autovalores normalizados que para $q=6$ temos um único autovalor acima de $q / \sqrt{n}$, e para $q=1$ temos cinco. Os valores da curvatura normal conformal máxima para esses esquemas de perturbação são: 0,856 e 0,884, respectivamente. Recorde que esses valores podem ser interpretados como a proporção do afastamento da verossimilhança que é explicada pelos autovetores q-influentes. Os valores de corte 2b, para estes casos são 0,037 e 0,076, respectivamente. Por outro lado, a curvatura normal conformal máxima quando consideramos o esquema de perturbação da covariável IMC é 0,997. Podemos observar na Figura 5.12(a) que para $q=1$ existe um único autovalor normalizado acima de $q / \sqrt{n}$, sendo que os gráficos 5.12 (b) e 5.12 (c) mostram as contribuições agregadas de um $(q=1)$ e de todos $(q=0)$ os autovetores. O ponto de corte tal como é sugerido por Poon e Poon (1999) para este esquema de perturbação é 0,084.

A partir do análise de resíduos e de influência concluímos que existem quatro pontos aberrantes e/o influentes, $\sharp 31, \sharp 74, \sharp 131$ e $\sharp 163$. Retiramos uma a uma e conjuntamente as observações consideradas como influentes e aberrantes, obtendo as estimativas, o erros padrão e o desvio relativo percentual das estimativas dos parâmetros estimados, tal como mostra a Tabela 5.10. A partir dos resultados na Tabela 5.10 notamos que as estimativas dos parâmetros tiveram um forte impacto quando a observação $\sharp 163$ é retirada. Em consequência disso, também quando retiramos conjuntamente os casos $\sharp 131$ e $\sharp 163$. Concluímos que a observação detectada como aberrante ou influente 


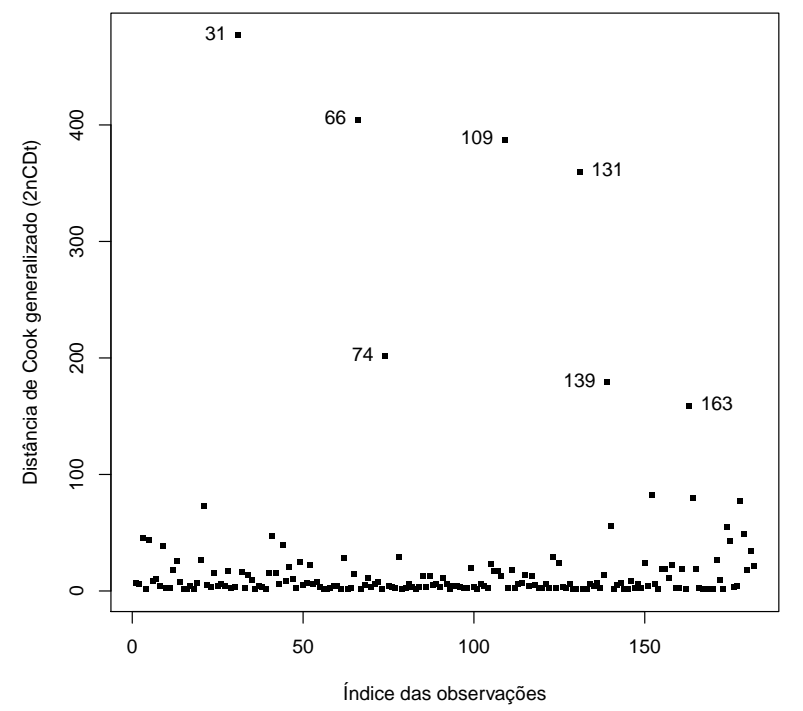

(a)

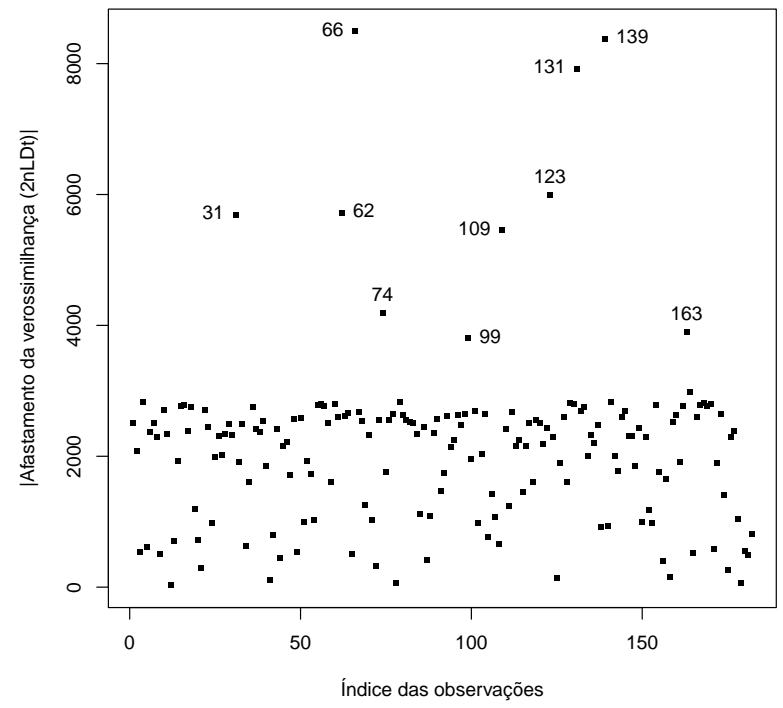

(b)

Figura 5.9: Medidas de influência global, (a) distância de Cook generalizado e (b) afastamento da verossimilhança.

que afeta resultados inferenciais do modelo é o caso $\sharp 163$.

Para avaliar a adequabilidade do modelo de regressão beta com erros de medida aos dados aqui, apresentamos a seguir, gráficos normais de probabilidades com envelope simulado. O gráfico da Figura 5.13(a) é construído considerando todas as observações. O gráfico da Figura 5.13(b) é construído retirando a observação $\sharp 163$, sendo esta aquela que apresentou maior desvio relativo percentual. Como era de se esperar o modelo de regressão beta com erros de medida ajusta-se bem ao conjunto de dados, quando consideramos todos os dados e quando retiramos a observação $\sharp 163$. Deixamos a critério do pesquisador a retirada ou não da observação $\sharp 163$. 


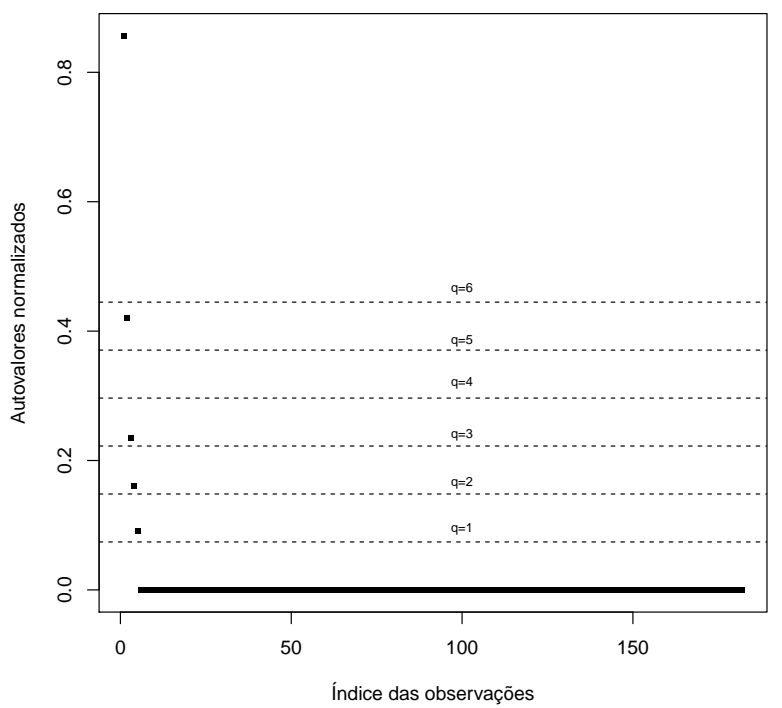

(a)

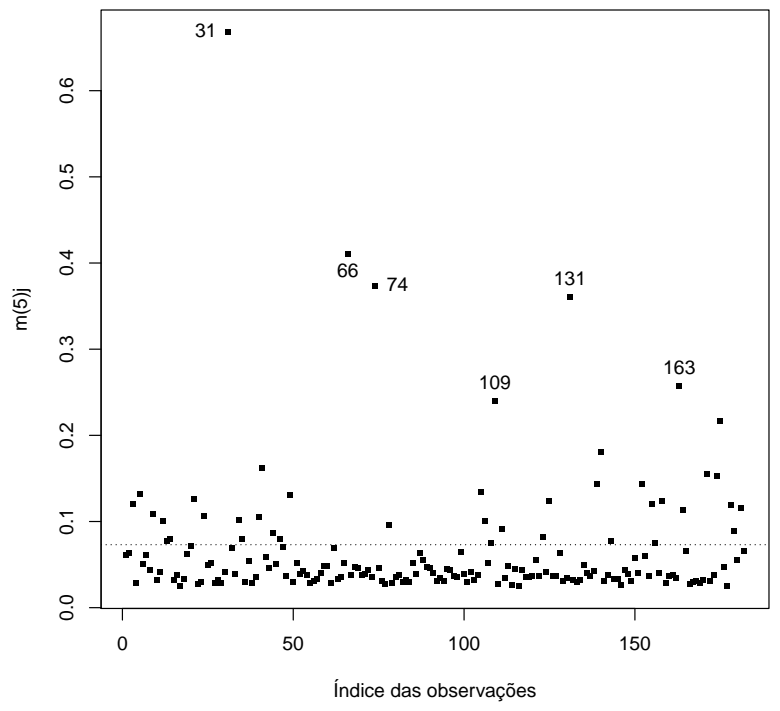

(c)

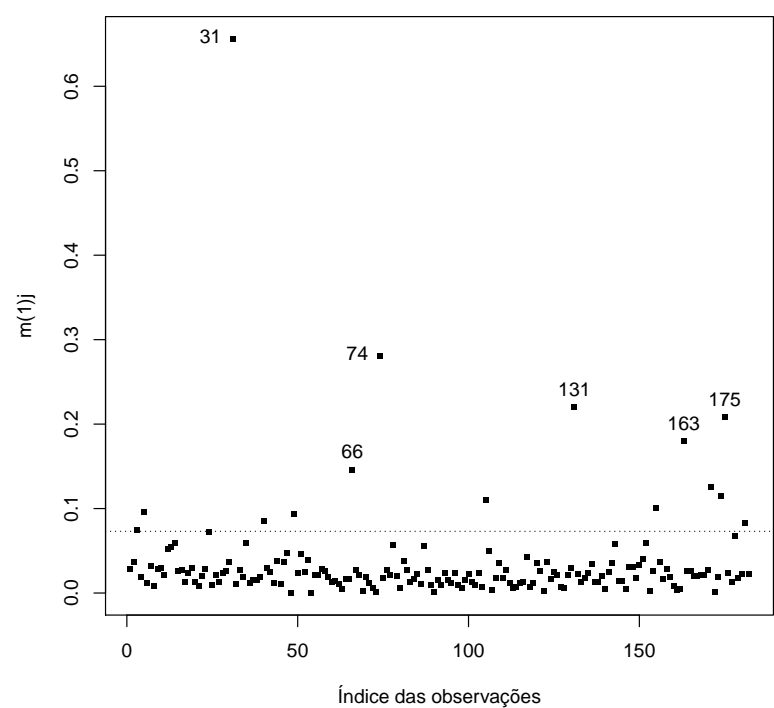

(b)

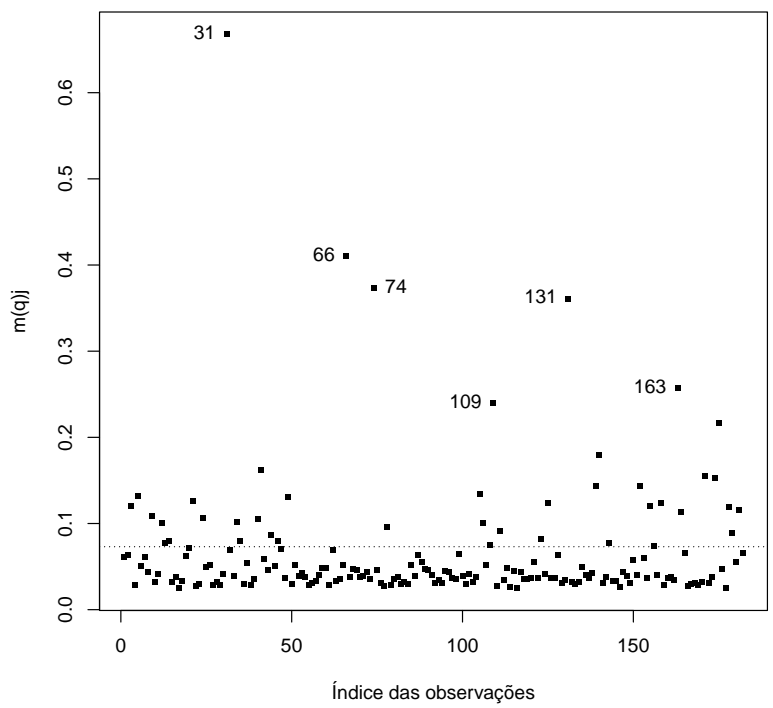

(d)

Figura 5.10: (a) Autovalores normalizados em módulo com valores de q, (b) influência devida à contribuição agregada do autovetor 1-influente, influência devida à contribuição agregada do autovetor 5-influente (c) e (d) influência devida à contribuição agregada de todos os autovetores, $m[q]_{j}$ para o esquema de perturbação de casos. 


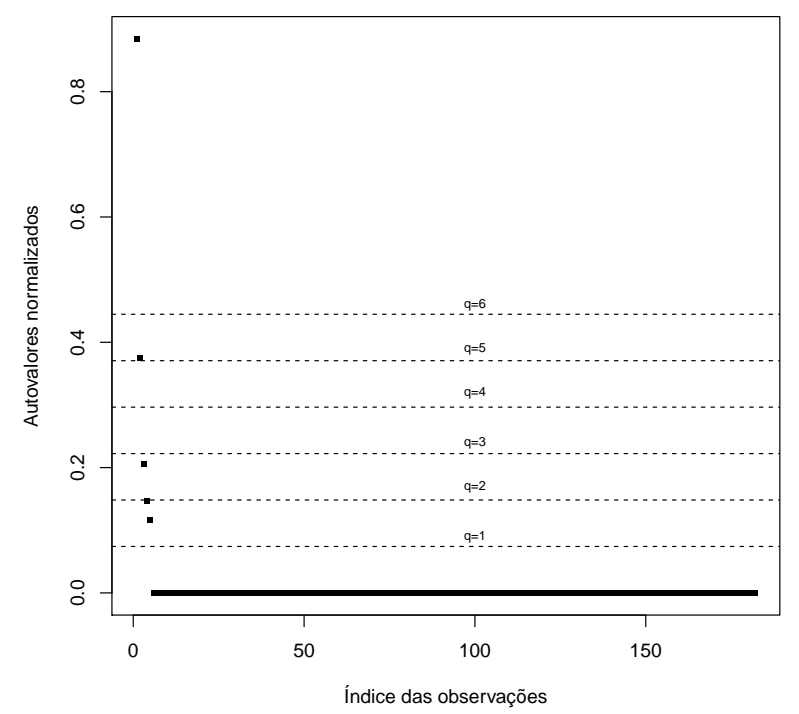

(a)

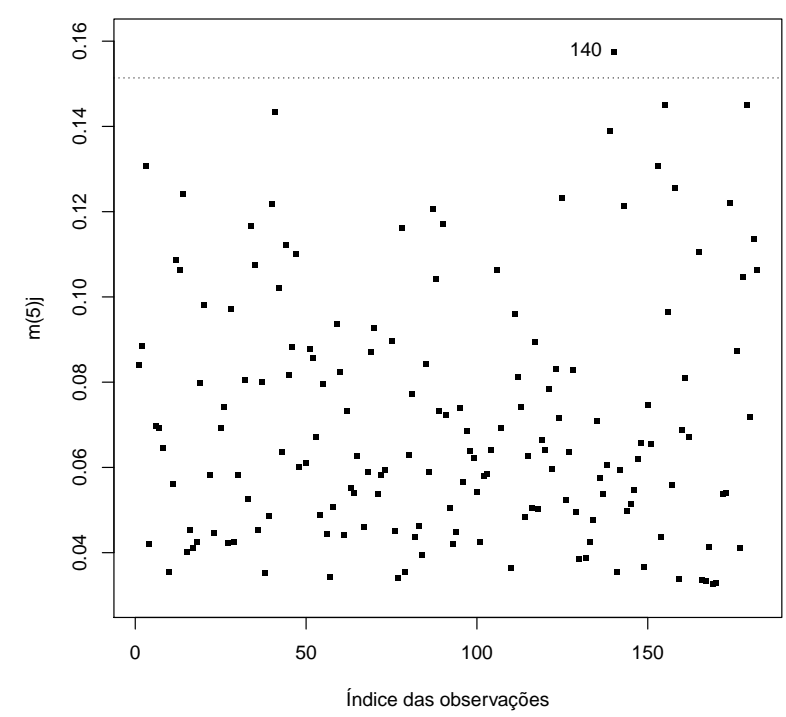

(c)

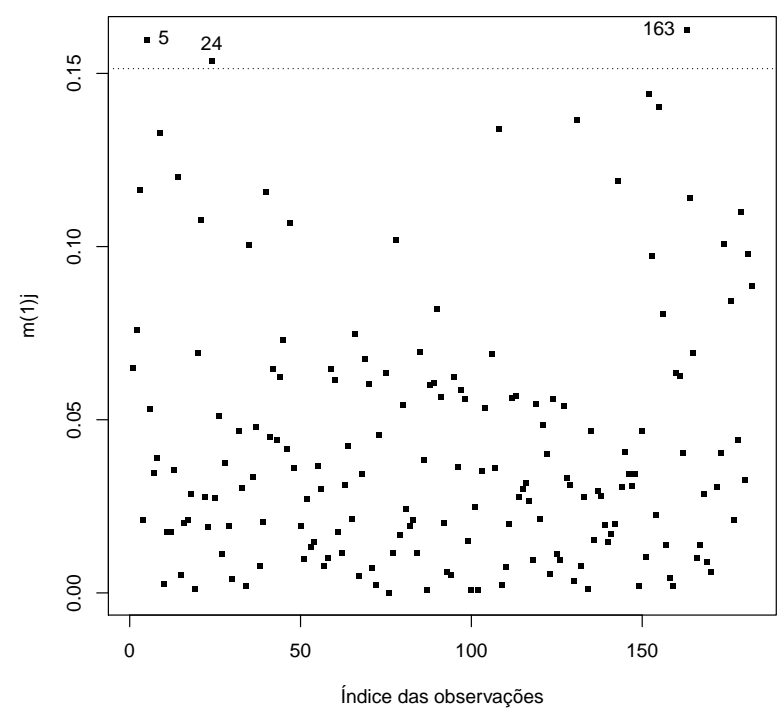

(b)

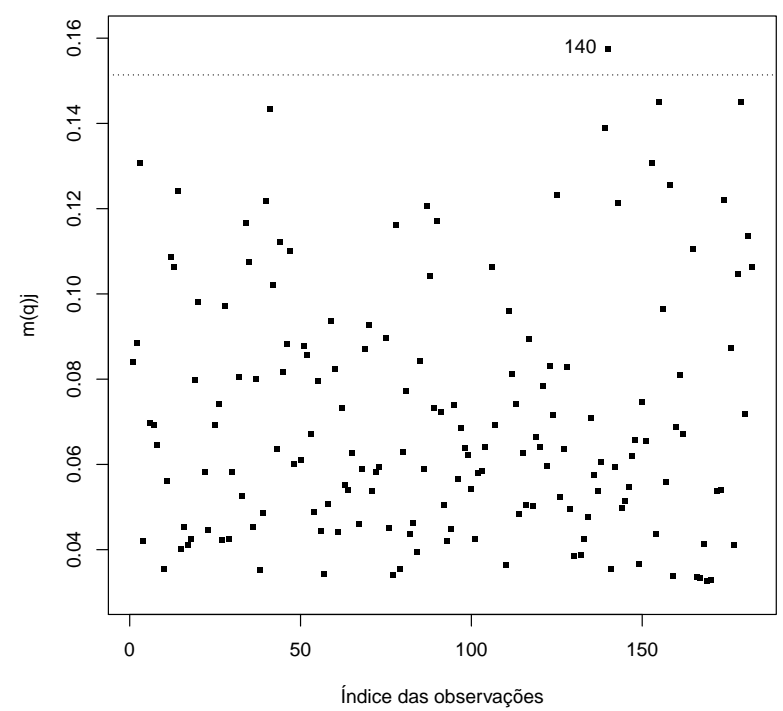

(d)

Figura 5.11: (a) Autovalores normalizados em módulo com valores de q, (b) influência devida à contribuição agregada do autovetor 1-influente, influência devida à contribuição agregada do autovetor 5-influente (c) e (d) influência devida à contribuição agregada de todos os autovetores, $m[q]_{j}$ para o esquema de perturbação de casos. 


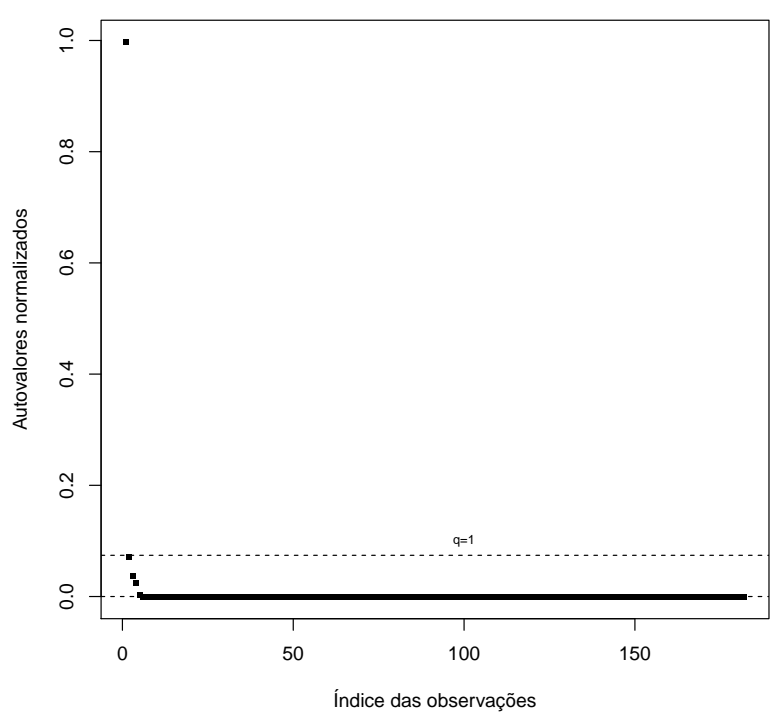

(a)

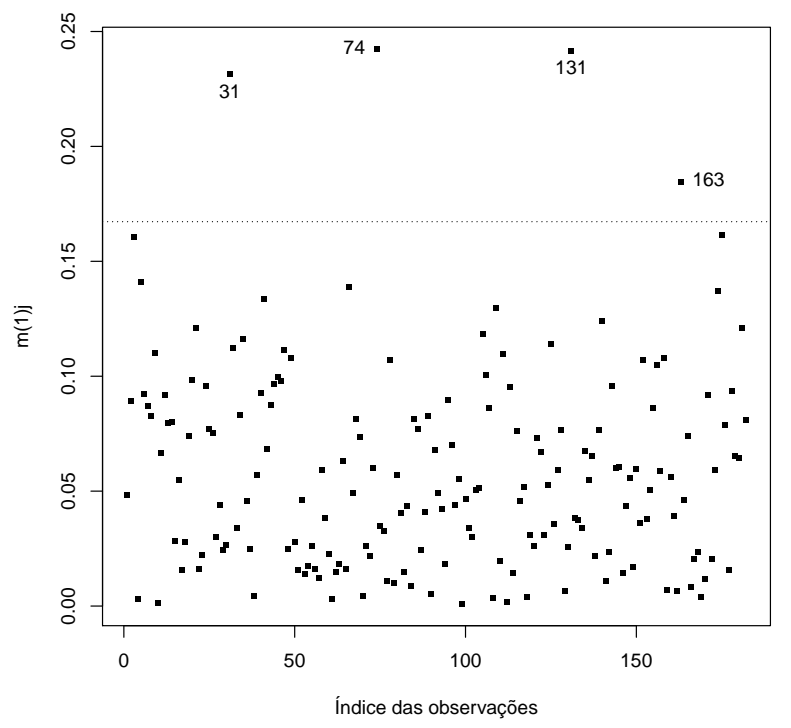

(b)

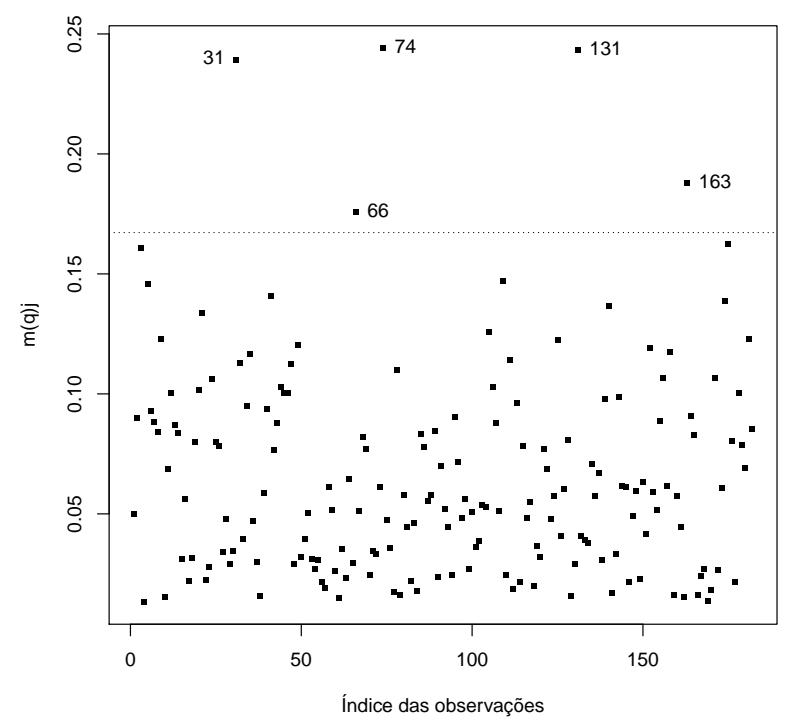

(c)

Figura 5.12: (a) Autovalores normalizados em módulo com valores de q, (b) influência devida à contribuição agregada do autovetor 1-influente e (c) influência devida à contribuição agregada de todos os autovetores, $m[q]_{j}$ para o esquema de perturbação de casos. 
Tabela 5.10: Estimativas (E), erros padrão (EP) e desvio relativo percentual (DRP) obtidos pelo método de máxima pseudo-verossimilhança.

\begin{tabular}{|c|c|c|c|c|}
\hline Exclusão & $\widehat{\boldsymbol{\theta}}$ & $E$ & $E P$ & $\operatorname{DRP}(\%)$ \\
\hline \multirow{5}{*}{ Nenhuma } & $\alpha_{0}$ & $-0,330$ & 0,119 & - \\
\hline & $\alpha_{1}$ & 0,010 & 0,004 & - \\
\hline & $\beta$ & 0,102 & 0,060 & - \\
\hline & $\gamma_{0}$ & 5,127 & 0,449 & - \\
\hline & $\lambda$ & $-0,620$ & 0,305 & - \\
\hline \multirow{5}{*}{$\sharp 31$} & $\alpha_{0}$ & $-0,322$ & 0,119 & 2,424 \\
\hline & $\alpha_{1}$ & 0,010 & 0,004 & 0,000 \\
\hline & $\beta$ & 0,105 & 0,060 & $-2,941$ \\
\hline & $\gamma_{0}$ & 5,125 & 0,449 & 0,039 \\
\hline & $\lambda$ & $-0,617$ & 0,305 & 0,484 \\
\hline \multirow{5}{*}{$\sharp 74$} & $\alpha_{0}$ & $-0,328$ & 0,119 & 0,606 \\
\hline & $\alpha_{1}$ & 0,010 & 0,004 & 0,000 \\
\hline & $\beta$ & 0,101 & 0,061 & 0,989 \\
\hline & $\gamma_{0}$ & 5,100 & 0,450 & 0,527 \\
\hline & $\lambda$ & $-0,603$ & 0,305 & 2,742 \\
\hline \multirow{5}{*}{$\sharp 31$ e $\sharp 74$} & $\alpha_{0}$ & $-0,320$ & 0,120 & 3,030 \\
\hline & $\alpha_{1}$ & 0,010 & 0,004 & 0,000 \\
\hline & $\beta$ & 0,103 & 0,061 & $-0,980$ \\
\hline & $\gamma_{0}$ & 5,099 & 0,450 & 0,546 \\
\hline & $\lambda$ & $-0,601$ & 0,305 & 3,065 \\
\hline \multirow{5}{*}{$\sharp 131$} & $\alpha_{0}$ & $-0,330$ & 0,119 & 0,000 \\
\hline & $\alpha_{1}$ & 0,010 & 0,004 & 0,000 \\
\hline & $\beta$ & 0,102 & 0,060 & 0,000 \\
\hline & $\gamma_{0}$ & 5,127 & 0,449 & 0,000 \\
\hline & $\lambda$ & $-0,624$ & 0,304 & $-0,645$ \\
\hline \multirow{5}{*}{$\sharp 163$} & $\alpha_{0}$ & $-0,364$ & 0,122 & $-10,303$ \\
\hline & $\alpha_{1}$ & 0,009 & 0,004 & 10,000 \\
\hline & $\beta$ & 0,117 & 0,061 & $-14,706$ \\
\hline & $\gamma_{0}$ & 5,120 & 0,474 & 0,137 \\
\hline & $\lambda$ & $-0,614$ & 0,320 & 0,968 \\
\hline \multirow{5}{*}{$\sharp 131$ e $\sharp 163$} & $\alpha_{0}$ & $-0,364$ & 0,122 & $-10,303$ \\
\hline & $\alpha_{1}$ & 0,009 & 0,004 & 10,000 \\
\hline & $\beta$ & 0,117 & 0,061 & $-14,706$ \\
\hline & $\gamma_{0}$ & 5,120 & 0,473 & 0,137 \\
\hline & $\lambda$ & $-0,618$ & 0,319 & 0,323 \\
\hline
\end{tabular}




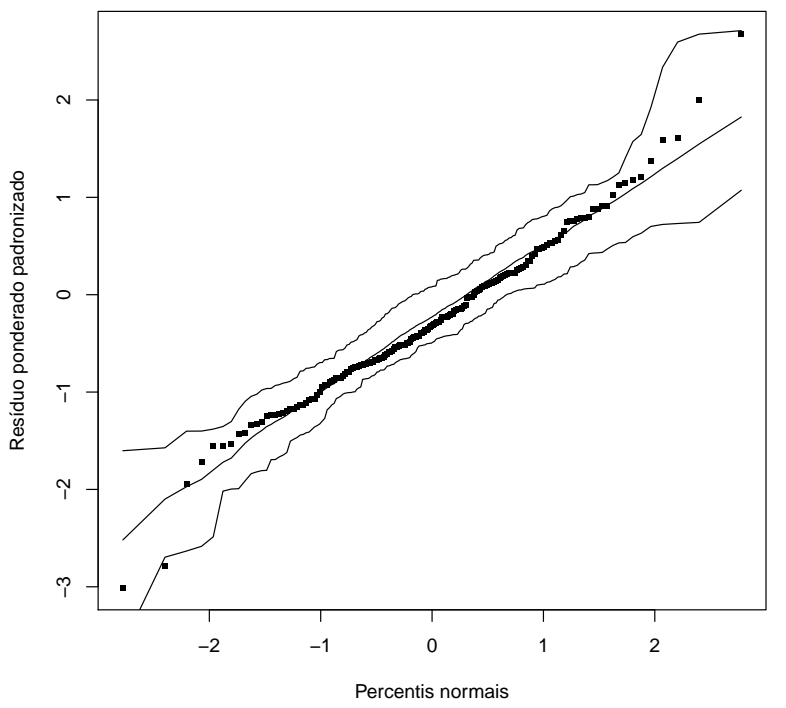

(a)

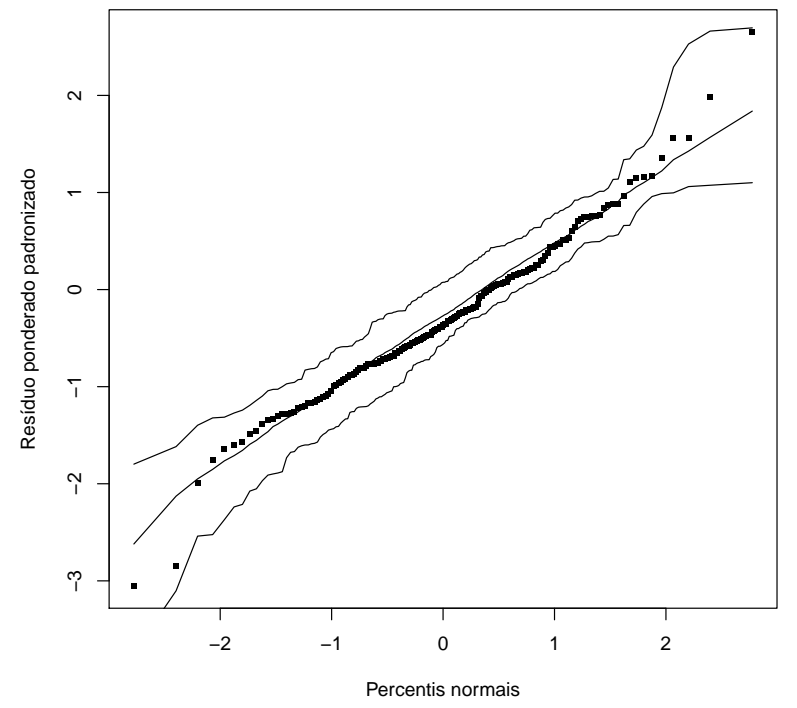

(b)

Figura 5.13: Gráficos de envelope simulado para (a) todas as observações e (b) quando retiramos a observação $\sharp 163$ que apresento maior desvio relativo percentual. 


\section{Capítulo 6}

\section{Considerações finais}

Neste trabalho desenvolvemos vários aspectos de inferência e diagnóstico para o modelo de regressão beta com erros de medida nas covariáveis. Apresentamos a seguir as principais conclusões.

- No Capítulo 2 apresentamos um modelo de regressão beta na presença de erros de medida nas covariáveis. Apresentamos os métodos de máxima verossimilhança aproximada, pseudoverossimilhança e calibração da regressão para estimar os parâmetros. Avaliamos numericamente o comportamento dos estimadores dos parâmetros calculando a média, viés e raiz do erro quadrático médio de 10000 réplicas Monte Carlo. Os resultados evidenciaram superioridade dos métodos de máxima verossimilhança aproximada e pseudo-verossimilhança aproximada. Em termos computacionais o método da pseudo-verossimilhança requer menos esforço computacional e é mais flexível.

- No Capítulo 3 encontramos as distribuições assintóticas dos estimadores obtidos pelos métodos de máxima verossimilhança aproximada, pseudo-verossimilhança e calibração da regressão. Utilizamos as estatísticas da razão de verossimilhanças e gradiente para testar hipóteses.

- No Capítulo 4 desenvolvemos o resíduo ponderado padronizado proposto por Espinheira et al. (2008a) para os modelos de regressão beta com erros de medida. Avaliamos numericamente o desempenho do resíduo proposto na presença de erros de medida. A distribuição empírica destes resíduos na presença de erros de medida é próxima da distribuição normal padrão quando utilizamos os métodos de máxima verossimilhança e pseudo-verossimilhança aproximada. $\mathrm{O}$ mesmo não ocorre quando utilizamos o método de calibração da regressão para tamanhos de amostra pequena e variância do erro de medição grande. Para a classe de modelos de regressão beta com erros de medida, desenvolvemos medidas de influência global, local e total.

- No Capítulo 5 consideramos dois conjuntos de dados reais para avaliar as metodologias propostas nos Capítulos 2 a 4 . Utilizamos o método de bootstrap como alternativa para obter erros padrão, $p$-valores e intervalos de confiança.

\subsection{Trabalhos futuros}

Como possíveis trabalhos futuros propomos:

- utilizar o enfoque funcional para contornar a presença de erros de medida quando a variável de interesse é contínua no intervalo $(0,1)$;

- utilizar o método de estimação EM-Monte Carlo como alternativa de estimação dos parâmetros; 
- utilizar a técnica de estimação SIMEX;

- como o método de estimação de calibração da regressão é muito útil pela simplicidade computacional, mas produz viés considerável, como evidenciado em nossas simulações, pretendemos desenvolver técnicas de correção de viés para este método;

- desenvolver metodologias de inferência sob o paradigma bayesiano para o modelo proposto nesta tese;

- considerar erros de medida na variável resposta;

- implementar os resultados na linguagem de programação R. 


\section{Apêndice A}

\section{A.1 Quadratura de Gauss-Hermite}

Esta seção do Apêndice é baseada na dissertação de mestrado de Peixoto (2008). É frequente que as integrais definidas sejam usadas como ferramentas essenciais na resolução de problemas de natureza matemática, física e computacional, entre outras. No entanto, é comum nos depararmos com integrais de funções que não possuem antiderivada explícita ou cuja antiderivada não é simples de se obter. Nesse sentido, é indispensável recorrer a métodos de aproximação. Os métodos que aproximam integrais envolvem combinações lineares de avaliação do integrando, isto é,

$$
\begin{aligned}
\int_{a}^{b} f(x) d x & \approx c_{1} f\left(x_{1}\right)+c_{2} f\left(x_{2}\right)+\ldots+c_{q} f\left(x_{q}\right), \quad-\infty<a<b<\infty \\
& =\sum_{i=1}^{q} c_{i} f\left(x_{i}\right)
\end{aligned}
$$

em que os argumentos $x_{1}, x_{2}, \ldots, x_{q}$, são chamados de nós ou abscissas e $c_{1}, \ldots, c_{q}$ os respectivos coeficientes que pertencem ao conjunto dos números reais. A equação (A.1) consiste em aproximar a integral pela soma das áreas de retângulos de base $c_{i}$ e altura $f\left(x_{i}\right)$, para $i=1,2, \ldots, q$, tal como mostra a Figura A.1. Em um sentido mais amplo, este somatório é uma quadratura. O alemão Carl Friedrich Gauss (1777-1855) aperfeiçoou as técnicas de aproximação de Newton-Cotes ${ }^{1}$ e formulou a regra de quadratura. A quadratura de Gauss considera as abscissas $x_{i}$ da equação (A.1) como sendo as raízes de um polinômio ortogonal.

Uma sequência de polinômios $\left\{p_{q}(x)\right\}_{q=0}^{\infty}$, pertencentes ao espaço de todos os polinômios algébricos de um grau menor ou igual a $q$, é uma sequência de polinômios ortogonais em relação a uma função peso $\omega(x)$ definido no intervalo real $[a, b]^{2}$, se

- $p_{q}(x)=\sum_{i=0}^{q} A_{q, i} x^{i}$ possuir grau exatamente $q$, isto é, $A_{q, q} \neq 0$,

- $<p_{q_{1}}(x), p_{q_{2}}(x)>=\int_{a}^{b} p_{q_{1}}(x) p_{q_{2}}(x) d f(x)=\int_{a}^{b} p_{q_{1}}(x) p_{q_{2}}(x) \omega(x) d x=0$,

sendo $f(x)$ uma função contínua. Os polinômios ortogonais comuns na literatura são os polinômios de Jacobi ( incluindo casos particulares de Legendre, de Chebyshev de $1^{\underline{a}}$ e $2^{\underline{a}}$ espécie e de Gegenbauer), de Hermite e de Leguerre generalizado. Neste trabalho, utilizamos polinômios ortogonais de Hermite.

Seja uma sequência de polinômios $\left\{p_{q}(x)\right\}_{q=0}^{\infty}$ definida com $\omega(x)=e^{-x^{2}}$ sobre o intervalo $(-\infty, \infty)$. Então, o polinômio de Hermite de grau $q$, que denotaremos por $H_{q}(x)$ (mais detalhes em

\footnotetext{
${ }^{1}$ Este resultado consta de sua célebre obra: Methodus nova integralium valores per approxinationem inveniend: Commentationes Societatis Regiae Scientarium Gottingensis Recentiores, 1814

${ }^{2}$ Caso $a=-\infty$ ou $b=\infty$, assume-se, sem perda de generalidade, que o intervalo é aberto neste(s) extremo(s)
} 


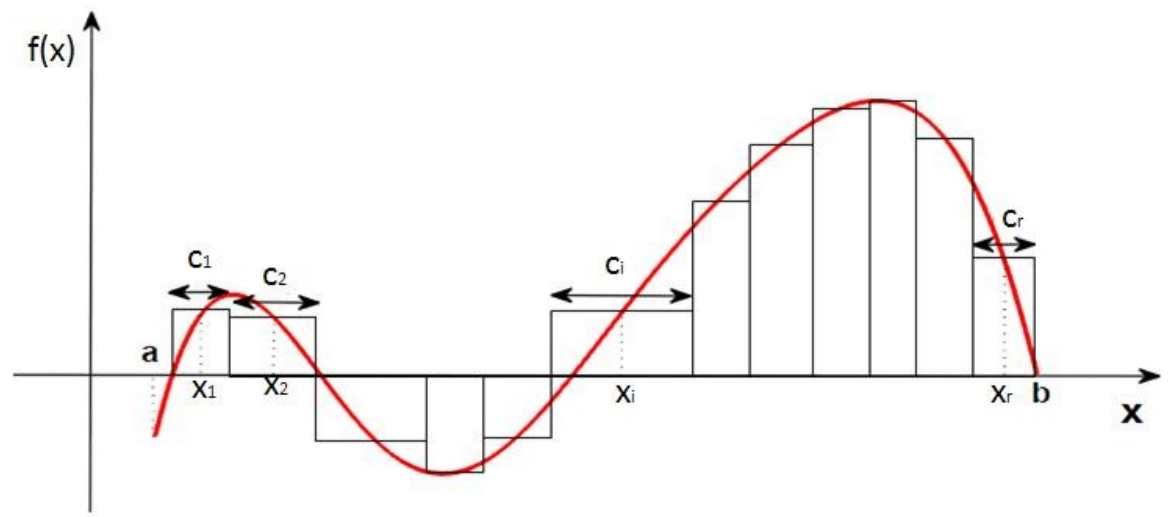

Figura A.1: Aproximação da integral por retângulos de base $c_{i}$ e altura $f\left(x_{i}\right)$. Adaptado da Figura 2 de Peixoto (2008).

(Abramowitz e Stegun, 1972, Cap.22)), é dado por

$$
H_{q}(x)=(-1)^{q} e^{x^{2}} \frac{d^{q}}{d x^{q}}\left\{e^{-x^{2}}\right\},
$$

sendo que os primeiros seis polinômios de Hermite são

$$
\begin{aligned}
& H_{0}(x)=1, \\
& H_{1}(x)=2 x \\
& H_{2}(x)=4 x^{2}-2, \\
& H_{3}(x)=8 x^{3}-12 x, \\
& H_{4}(x)=16 x^{4}-48 x^{2}+12, \\
& H_{5}(x)=32 x^{5}-160 x^{3}+120 x .
\end{aligned}
$$

É possível verificar que as raízes do $q$-ésimo polinômios pertencem ao conjunto dos números reais, são distintas e simétricas com relação à origem, pois $\omega(x)=e^{-x^{2}}$ é uma função par. Tabelas para os zeros dos q-ésimos polinômios de Hermite podem ser encontrados em Abramowitz e Stegun (1972, Cap. 22). Podemos utilizar também a linguagem de programação R (versão 2.23.1) para a determinação destas raízes a partir da função ghq implementada no pacote glmmML.

O polinômio de Hermite $H_{q}(x)$ definido com $\omega(x)=e^{-x^{2}}$, está diretamente associado com o método de quadratura de Gauss. Esta associação é conhecida como a quadratura de Gauss-Hermite, 
que nos permite aproximar integrais da forma $\int_{-\infty}^{\infty} e^{-x^{2}} f(x) d x$, da seguinte maneira

$$
\int_{-\infty}^{\infty} e^{-x^{2}} f(x) d x=\sum_{i=1}^{q} \nu_{i} f\left(s_{i}\right)+E_{q}
$$

em que $E_{q}$ é o erro de uma quadratura, $s_{i}, i=1, \ldots, q$ são as raízes de $H_{q}(x)$ e $\nu_{i}$ são pesos associados a cada raiz, dados por

$$
\nu_{i}=\frac{2^{q-1} q ! \sqrt{\pi}}{q^{2}\left[H_{q-1}\left(s_{i}\right)\right]^{2}}
$$

É por intermédio de uma estimativa de $E_{q}$ que o cálculo da quadratura, torna-se confiável. Existe uma forma rápida e prática para estimar $E_{q}$, através do cálculo do modulo da diferença entre os resultados das quadraturas com $q_{1}$ e $q_{2}$ abscissas, sendo $q_{2}>q_{1}$, isto é

$$
E_{q} \approx\left|\sum_{j=1}^{q_{2}} \nu_{j} f\left(s_{j}\right)-\sum_{k=1}^{q_{1}} \nu_{k} f\left(s_{k}\right)\right|, \quad q_{2}>q_{1} .
$$

Uma característica do método de integração por quadratura de Gauss-Hermite é que quando $f(\cdot)$ for um polinômio de grau $2 q-1$, a aproximação se torna exata. Em outras palavras, $E_{q}=0$ quando o número de pontos de quadratura for suficientemente grande.

\section{A.2 Vetor escore e matriz de informação observada aproximada}

\section{A.2.1 Vetor escore}

Apresentamos nesta seção alguns passos do desenvolvimento das primeiras derivadas de $\ell_{a}(\mathbf{\Psi})$ em relação aos parâmetros de $\boldsymbol{\Psi}$, ou seja a função escore. O logaritmo da função de verossimilhança aproximada é dado por

$$
\ell_{a}(\boldsymbol{\Psi})=\sum_{t=1}^{n} \ell_{1 t}\left(\boldsymbol{\xi}, \sigma_{e}^{2}\right)+\sum_{t=1}^{n} \ell_{2 t}\left(\boldsymbol{\theta}, \boldsymbol{\xi}, \sigma_{e}^{2}\right)
$$

em que

$$
\begin{aligned}
& \ell_{1 t}\left(\boldsymbol{\xi}, \sigma_{e}^{2}\right)=-\frac{1}{2} \log \left[2 \pi\left(\sigma_{x}^{2}+\sigma_{e}^{2}\right)\right]-\frac{1}{2\left(\sigma_{x}^{2}+\sigma_{e}^{2}\right)}\left(w_{t}-\mu_{t}\right)^{2}, \\
& \ell_{2 t}\left(\boldsymbol{\theta}, \boldsymbol{\xi}, \sigma_{e}^{2}\right)=\log \sum_{q=1}^{Q} \frac{\nu_{q}}{\sqrt{\pi}} \exp \left\{\ell_{t q}\left(\mu_{t q} ; \phi_{t q}\right)\right\}, \\
& \ell_{t q}\left(\mu_{t q} ; \phi_{t q}\right)=\log \Gamma\left(\phi_{t q}\right)-\log \Gamma\left(\mu_{t q} \phi_{t q}\right)-\log \Gamma\left[\left(1-\mu_{t q}\right) \phi_{t q}\right]+\left(\mu_{t q} \phi_{t q}-1\right) \log \left(y_{t}\right)+ \\
& {\left[\left(1-\mu_{t q}\right) \phi_{t q}-1\right] \log \left(1-y_{t}\right) \text {, }} \\
& g\left(\mu_{t q}\right)=\mathbf{z}_{t}^{\top} \boldsymbol{\alpha}+x_{t}^{*} \beta, \\
& h\left(\phi_{t q}\right)=\mathbf{v}_{t}^{\top} \gamma+x_{t}^{*} \lambda \text {, } \\
& x_{t}^{*}=\mu_{x_{t} \mid w_{t}}+\sqrt{2 \sigma_{x_{t} \mid w_{t}}^{2}} s_{q}, \\
& \mu_{x_{t} \mid w_{t}}=\mu_{x}+k_{x}\left(w_{t}-\mu_{x}\right) \\
& \sigma_{x_{t} \mid w_{t}}^{2}=\sigma_{e}^{2} k_{x} \text {, } \\
& k_{x}=\frac{\sigma_{x}^{2}}{\sigma_{x}^{2}+\sigma_{e}^{2}} .
\end{aligned}
$$


Para $i=1,2, \ldots, p$, a função escore aproximada para cada $\alpha_{i}$ é dada por

$$
\frac{\partial \ell_{a}(\mathbf{\Psi})}{\partial \alpha_{i}}=U_{\alpha_{i}}=\sum_{t=1}^{n} b_{t} a_{t \alpha_{i}}
$$

em que

$$
\begin{aligned}
b_{t} & =\left(\sum_{q=1}^{Q} \frac{\nu_{q}}{\sqrt{\pi}} \exp \left\{\ell_{t q}\left(\mu_{t q}, \phi_{t q}\right)\right\}\right)^{-1}, \\
a_{t \alpha_{i}} & =\sum_{q=1}^{Q} \frac{\nu_{q}}{\sqrt{\pi}} \exp \left\{\ell_{t q}\left(\mu_{t q}, \phi_{t q}\right)\right\} \dot{\ell}_{t q\left(\alpha_{i}\right)}\left(\mu_{t q}, \phi_{t q}\right), \\
\dot{\ell}_{t q\left(\alpha_{i}\right)}\left(\mu_{t q}, \phi_{t q}\right) & =\phi_{t}\left(y_{t}^{*}-\mu_{t q}^{*}\right) \frac{1}{g^{\prime}\left(\mu_{t q}\right)} z_{t i}, \\
\mu_{t q}^{*} & =\psi\left(\mu_{t q} \phi_{t q}\right)-\psi\left[\left(1-\mu_{t q}\right) \phi_{t q}\right], \\
y_{t}^{*} & =\log \left(\frac{y_{t}}{1-y_{t}}\right),
\end{aligned}
$$

em que $\psi(\cdot)$ é a função digama. Em forma matricial o vetor escore para o parâmetro $\boldsymbol{\alpha}$ pode ser expresso como $U_{\alpha}=A_{\alpha}^{\top} B$, sendo que $A_{\alpha}^{\top}=\operatorname{diag}\left(a_{t \alpha_{1}}, a_{t \alpha_{2}}, \ldots, a_{t \alpha_{p}}\right)$ e $B=\left(b_{1}, b_{2}, \ldots, b_{n}\right)^{\top}$. A função escore para $\beta$, parâmetro correspondente à covariável medida com erro, é dada por

$$
\frac{\partial \ell_{a}(\mathbf{\Psi})}{\partial \beta}=U_{\beta}=\sum_{t=1}^{n} b_{t} a_{t \beta},
$$

em que

$$
\begin{aligned}
a_{t \beta} & =\sum_{q=1}^{Q} \frac{\nu_{q}}{\sqrt{\pi}} \exp \left\{\ell_{t q}(\Psi)\right\} \dot{\ell}_{t q(\beta)}\left(\mu_{t q}, \phi_{t q}\right), \\
\dot{\ell}_{t q(\beta)}\left(\mu_{t q}, \phi_{t q}\right) & =\phi_{t}\left(y_{t}^{*}-\mu_{t q}^{*}\right) \frac{1}{g^{\prime}\left(\mu_{t q}\right)} x_{t}^{*} .
\end{aligned}
$$

Em forma matricial o vetor escore para o parâmetro $\beta$ é dado por $U_{\beta}=A_{\beta}^{\top} B$, sendo $A_{\beta}=$ $\left(a_{1 \beta}, a_{2 \beta}, \ldots, a_{n \beta}\right)^{\top}$.

Agora derivemos $\ell_{a}(\Psi)$ com relação aos parâmetros de precisão, $\gamma_{j}$, para todo $j=1,2, \ldots, \breve{q}$, e $\lambda$. Temos que

$$
\begin{aligned}
& \frac{\partial \ell_{a}(\boldsymbol{\Psi})}{\partial \gamma_{j}}=U_{\gamma_{j}}=\sum_{t=1}^{n} b_{t} a_{t \gamma_{j}}, \\
& \frac{\partial \ell_{a}(\boldsymbol{\Psi})}{\partial \lambda}=U_{\lambda}=\sum_{t=1}^{n} b_{t} a_{t \lambda},
\end{aligned}
$$

em que

$$
\begin{aligned}
a_{t \gamma_{j}} & =\sum_{q=1}^{Q} \frac{\nu_{q}}{\sqrt{\pi}} \exp \left\{\ell_{t q}\left(\mu_{t q}, \mu_{t q}\right)\right\} \dot{\ell}_{t q\left(\gamma_{j}\right)}\left(\mu_{t q}, \mu_{t q}\right), \\
a_{t \lambda} & =\sum_{q=1}^{Q} \exp \left\{\ell_{t q}\left(\mu_{t q}, \phi_{t q}\right)\right\} \dot{\ell}_{t q(\lambda)}\left(\mu_{t q}, \phi_{t q}\right), \\
\dot{\ell}_{t q\left(\gamma_{j}\right)}\left(\mu_{t q}, \mu_{t q}\right) & =a_{t} \frac{1}{h^{\prime}\left(\phi_{t}\right)} v_{t j}, \\
\dot{\ell}_{t q(\lambda)}\left(\mu_{t q}, \phi_{t q}\right) & =a_{t} \frac{1}{h^{\prime}\left(\phi_{t}\right)} x_{t}^{*}, \\
a_{t} & =\mu_{t}\left(y_{t}^{*}-\mu_{t}^{*}\right)+\log \left(1-y_{t}\right)-\psi\left[\left(1-\mu_{t}\right) \phi_{t}\right]+\psi\left(\phi_{t}\right) .
\end{aligned}
$$


Os vetores escore com relação aos parâmetros $\gamma$ e $\lambda$ são definidos, respectivamente como $U_{\gamma}=A_{\gamma}^{\top} B$ e $U_{\lambda}=A_{\lambda}^{\top} B$, sendo $A_{\gamma}=\operatorname{diag}\left(a_{t \gamma_{1}}, \ldots, a_{t \gamma_{\breve{q}}}\right)$ e $A_{\lambda}=\left(a_{1 \lambda}, \ldots, a_{n \lambda}\right)^{\top}$. Finalmente, as derivadas do logaritmo da função verossimilhança aproximada em relação aos parâmetros $\mu_{x}$ e $\sigma_{x}^{2}$ são dadas por

$$
\begin{aligned}
& U_{\mu_{x}}=\frac{1}{\left(\sigma_{x}^{2}+\sigma_{e}^{2}\right)} \sum_{t=1}^{n}\left(w_{t}-\mu_{x}\right)+\sum_{t=1}^{n} b_{t} a_{t \mu_{x}}, \\
& U_{\sigma_{x}^{2}}=-\frac{1}{2\left(\sigma_{x}^{2}+\sigma_{e}^{2}\right)} \sum_{t=1}^{n}\left\{1-\frac{\left(w_{t}-\mu_{x}\right)^{2}}{\left(\sigma_{x}^{2}+\sigma_{e}^{2}\right)}\right\}+\sum_{t=1}^{n} b_{t} a_{t \sigma_{x}^{2}},
\end{aligned}
$$

em que

$$
\begin{aligned}
a_{t \mu_{x}} & =\sum_{q=1}^{Q} \frac{\nu_{q}}{\sqrt{\pi}} \exp \left\{\ell_{t q}\left(\mu_{t q}, \phi_{t q}\right)\right\} \dot{\ell}_{t q\left(\mu_{x}\right)}\left(\mu_{t q}, \phi_{t q}\right), \\
a_{t \sigma_{x}^{2}} & =\sum_{q=1}^{Q} \frac{\nu_{q}}{\sqrt{\pi}} \exp \left\{\ell_{t q}\left(\mu_{t q}, \phi_{t q}\right)\right\} \dot{\ell}_{t q\left(\sigma_{x}^{2}\right)}\left(\mu_{t q}, \phi_{t q}\right), \\
\dot{\ell}_{t q\left(\mu_{x}\right)}\left(\mu_{t q}, \phi_{t q}\right) & =\left(1-k_{x}\right)\left\{\beta \frac{\phi_{t q}\left(y_{t}^{*}-\mu_{t q}^{*}\right)}{g^{\prime}\left(\mu_{t q}\right)}+\lambda \frac{a_{t}}{h^{\prime}\left(\phi_{t q}\right)}\right\}, \\
\dot{\ell}_{t q\left(\sigma_{x}^{2}\right)}\left(\mu_{t q}, \phi_{t q}\right) & =\frac{\left(1-k_{x}\right)^{2}}{2 \sigma_{x_{t} \mid w_{t}}^{2}}\left\{\beta \frac{\phi_{t q}\left(y_{t}^{*}-\mu_{t q}^{*}\right)}{g^{\prime}\left(\mu_{t q}\right)}+\lambda \frac{a_{t}}{h^{\prime}\left(\phi_{t q}\right)}\right\} s_{q} .
\end{aligned}
$$

As equações (A.14) e (A.15) podem ser escritas matricialmente da seguinte forma: $U_{\mu_{x}}=W_{\mu_{x}}+$ $A_{\mu_{x}}^{\top} B$ e $U_{\sigma_{x}^{2}}=W_{\sigma_{x}^{2}}+A_{\sigma_{x}^{2}}^{\top} B$, em que $W_{\mu_{x}}$ e $W_{\sigma_{x}^{2}}$ são vetores que dependem da covariável medida com erro $w_{t}$ cujos $t$-ésimos elementos são dados por $\left(w_{t}-\mu_{x}\right) /\left(\sigma_{x}^{2}+\sigma_{e}^{2}\right)$ e $-\left[1-\left(w_{t}-\mu_{t q}\right)^{2} /\left(\sigma_{x}^{2}+\right.\right.$ $\left.\left.\sigma_{e}^{2}\right)\right] / 2\left[\sigma_{x}^{2}+\sigma_{e}^{2}\right]$, respectivamente. Ademais, $A_{\mu_{x}}=\left(a_{1 \mu_{x}}, \ldots, a_{n \mu_{x}}\right)^{\top}$ e $A_{\sigma_{x}^{2}}=\left(a_{1 \sigma_{x}^{2}}, \ldots, a_{n \sigma_{x}^{2}}\right)^{\top}$.

\section{A.2.2 Matriz de informação observada}

Apresentamos os elementos da matriz de informação observada dada por

$$
J(\Psi)=-\frac{\partial^{2} \ell(\mathbf{\Psi})}{\partial \Psi \partial \Psi^{\top}}
$$

com $\ell(\boldsymbol{\Psi})$ como em (A.4) e $\boldsymbol{\Psi}=\left(\boldsymbol{\alpha}^{\top}, \beta, \boldsymbol{\gamma}^{\top}, \lambda, \mu_{a}, \sigma_{x}^{2}\right)^{\top}$. A representação matricial da matriz de informação observada tem a forma

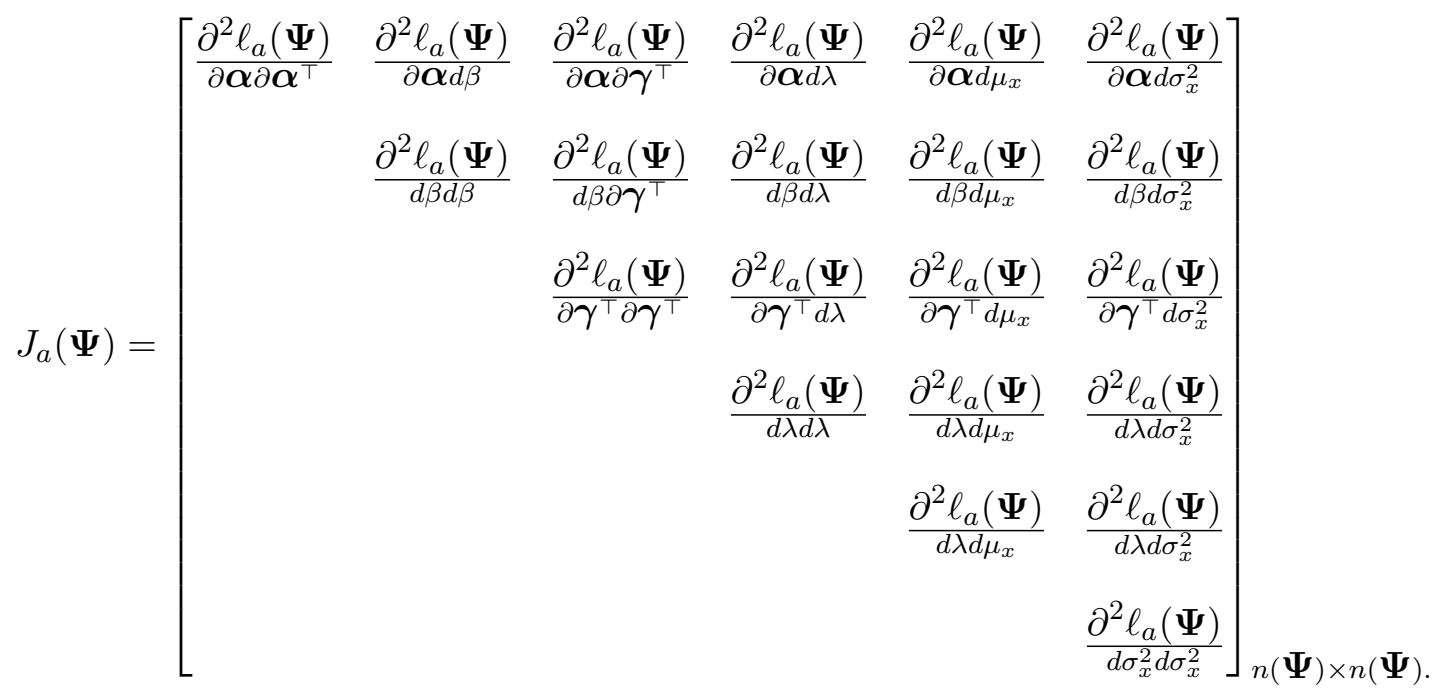


em que $n(\boldsymbol{\Psi})=(p+1)+(\breve{q}+1)+2$. A partir das primeiras derivadas de $\ell(\boldsymbol{\Psi})$ e resultados apresentados no Apêndice A.2.1, calculamos as expressões das derivadas segundas de $\ell(\Psi)$.

- Para $i, j=1,2, \ldots, p$ temos que $\frac{\partial^{2} \ell_{a}(\boldsymbol{\Psi})}{\partial \alpha_{i} \partial \alpha_{j}}=J_{a\left(\alpha_{i} \alpha_{j}\right)}=\sum_{t=1}^{n}\left\{\dot{b}_{t\left(\alpha_{j}\right)} a_{t \alpha_{i}}+b_{t} \dot{a}_{t \alpha_{i}\left(\alpha_{j}\right)}\right\}$, em que

$$
\begin{aligned}
\dot{b}_{t\left(\alpha_{j}\right)} & =-b_{t}^{-2} \sum_{q=1}^{Q} \frac{\nu_{q}}{\sqrt{\pi}} \exp \left\{\ell_{t q}\left(\mu_{t q}, \phi_{t q}\right)\right\} \dot{\ell}_{t q\left(\alpha_{j}\right)}\left(\mu_{t q}, \phi_{t q}\right), \\
\dot{\ell}_{t q\left(\alpha_{j}\right)}\left(\mu_{t q}, \phi_{t q}\right) & =\phi_{t q}\left(y^{*}-\mu_{t q}^{*}\right) \frac{1}{g^{\prime}\left(\mu_{t q}\right)} z_{t j}, \\
\dot{a}_{t \alpha_{i}\left(\alpha_{j}\right)} & =\sum_{q=1}^{Q} \frac{\nu_{q}}{\sqrt{\pi}} \exp \left\{\ell_{t q}\left(\mu_{t q}, \phi_{t q}\right)\right\}\left[\dot{\ell}_{t q\left(\alpha_{j}\right)}\left(\mu_{t q}, \phi_{t q}\right) \dot{\ell}_{t q\left(\alpha_{i}\right)}\left(\mu_{t q}, \phi_{t q}\right)+\right. \\
& \left.\ddot{\ell}_{t q\left(\alpha_{i}\right)\left(\alpha_{j}\right)}\left(\mu_{t q}, \phi_{t q}\right)\right], \\
\ddot{\ell}_{t q\left(\alpha_{i}\right)\left(\alpha_{j}\right)}\left(\mu_{t q}, \phi_{t q}\right)= & -\phi_{t q}\left\{u_{t}-\left(y_{t}^{*}-\mu_{t q}^{*}\right) \frac{g^{\prime \prime}\left(\mu_{t q}\right)}{\left\{g^{\prime}\left(\mu_{t q}\right)\right\}^{3}}\right\} z_{t i} z_{t j}, \\
u_{t}= & {\left[\phi_{t q}\left\{\Psi^{\prime}\left(\mu_{t q} \phi_{t q}\right)+\psi^{\prime}\left[\left(1-\mu_{t q}\right) \phi_{t q}\right]\right\}\right] \frac{1}{\left\{g^{\prime}\left(\mu_{t q}\right)\right\}^{2}}, }
\end{aligned}
$$

em que expressões como $a_{t \alpha_{i}}$ e $\dot{\ell}_{t q\left(\alpha_{j}\right)}\left(\mu_{t q}, \phi_{t q}\right)$ estão dadas em (A.6) e (A.7), respectivamente.

- Para $i=1,2, \ldots, p$ temos que $\frac{\partial^{2} \ell_{a}(\mathbf{\Psi})}{\partial \alpha_{i} \partial \beta}=J_{a\left(\alpha_{i} \beta\right)}=\sum_{t=1}^{n}\left\{\dot{b}_{t(\beta)} a_{t \alpha_{i}}+b_{t} \dot{a}_{t \alpha_{i}(\beta)}\right\}$,

em que

$$
\begin{aligned}
\dot{b}_{t(\beta)} & =-b_{t}^{-2} \sum_{q=1}^{Q} \frac{\nu_{q}}{\sqrt{\pi}} \exp \left\{\ell_{t q}\left(\mu_{t q}, \phi_{t q}\right)\right\} \dot{\ell}_{t q(\beta)}\left(\mu_{t q}, \phi_{t q}\right), \\
\dot{a}_{t \alpha_{i}(\beta)} & =\sum_{q=1}^{Q} \frac{\nu_{q}}{\sqrt{\pi}} \exp \left\{\ell_{t q}\left(\mu_{t q}, \phi_{t q}\right)\right\}\left[\dot{\ell}_{t q(\beta)}\left(\mu_{t q}, \phi_{t q}\right) \dot{\ell}_{t q\left(\alpha_{i}\right)}\left(\mu_{t q}, \phi_{t q}\right)+\ddot{\ell}_{t q\left(\alpha_{i}\right)(\beta)}\left(\mu_{t q}, \phi_{t q}\right)\right], \\
\ddot{\ell}_{t q\left(\alpha_{i}\right)(\beta)}\left(\mu_{t q}, \phi_{t q}\right) & =-\phi_{t q}\left\{u_{t}-\left(y_{t}^{*}-\mu_{t q}^{*} \frac{g^{\prime \prime}\left(\mu_{t q}\right)}{\left\{g^{\prime}\left(\mu_{t q}\right)\right\}^{3}}\right)\right\} z_{t i} x_{t}^{*},
\end{aligned}
$$

em que as expressões $\dot{\ell}_{t q(\beta)}\left(\mu_{t q}, \phi_{t q}\right)$ e $u_{t}$ são dadas em (A.10) e (A.19), respectivamente.

- Para $i=1,2, \ldots, p$ e $j=1,2, \ldots, \breve{q}$ temos que $\frac{\partial^{2} \ell_{a}(\mathbf{\Psi})}{\partial \alpha_{i} \partial \gamma_{j}}=J_{a\left(\alpha_{i} \gamma_{j}\right)}=\sum_{t=1}^{n}\left\{\dot{b}_{t\left(\gamma_{j}\right)} a_{t \alpha_{i}}+\right.$ $\left.b_{t} \dot{a}_{t \alpha_{i}\left(\gamma_{j}\right)}\right\}$

em que

$$
\begin{aligned}
\dot{b}_{t\left(\gamma_{j}\right)}= & -b_{t}^{-2} \sum_{q=1}^{Q} \frac{\nu_{q}}{\sqrt{\pi}} \exp \left\{\ell_{t q}\left(\mu_{t q}, \phi_{t q}\right)\right\} \dot{\ell}_{t q\left(\gamma_{j}\right)}\left(\mu_{t q}, \phi_{t q}\right), \\
\dot{a}_{t \alpha_{i}\left(\gamma_{j}\right)}= & \sum_{q=1}^{Q} \frac{\nu_{q}}{\sqrt{\pi}} \exp \left\{\ell_{t q}\left(\mu_{t q}, \phi_{t q}\right)\right\}\left[\dot{\ell}_{t q\left(\gamma_{j}\right)}\left(\mu_{t q}, \phi_{t q}\right) \dot{\ell}_{t q\left(\alpha_{i}\right)}\left(\mu_{t q}, \phi_{t q}\right)+\right. \\
& \left.\ddot{\ell}_{t q\left(\alpha_{i}\right)\left(\gamma_{j}\right)}\left(\mu_{t q}, \phi_{t q}\right)\right] \\
\ddot{\ell}_{t q\left(\alpha_{i}\right)\left(\gamma_{j}\right)}\left(\mu_{t q}, \phi_{t q}\right)= & \left\{\left(y_{t}^{*}-\mu_{t q}^{*}\right)-c_{t}\right\} \frac{1}{g^{\prime}\left(\mu_{t q}\right)} \frac{1}{h^{\prime}\left(\phi_{t q}\right)} z_{t i} v_{t j}, \\
c_{t}= & \phi_{t q}\left[\psi^{\prime}\left(\mu_{t q} \phi_{t q}\right) \mu_{t q}+\psi^{\prime}\left[\left(1-\mu_{t q}\right) \phi_{t q}\right]\left(1-\mu_{t q}\right)\right]
\end{aligned}
$$


cujas expressões de $\dot{\ell}_{t q\left(\gamma_{j}\right)}\left(\mu_{t q}, \phi_{t q}\right)$ e $a_{t}$ estão dadas em (A.11) e (A.13), respectivamente.

- Para $i=1,2, \ldots, p$ temos que $\frac{\partial^{2} \ell_{a}(\mathbf{\Psi})}{\partial \alpha_{i} \partial \lambda}=J_{a\left(\alpha_{i} \lambda\right)}=\sum_{t=1}^{n}\left\{\dot{b}_{t(\lambda)} a_{t \alpha_{i}}+b_{t} \dot{a}_{t \alpha_{i}(\lambda)}\right\}$, em que

$$
\begin{aligned}
\dot{b}_{t(\lambda)} & =-b_{t}^{-2} \sum_{q=1}^{Q} \frac{\nu_{q}}{\sqrt{\pi}} \exp \left\{\ell_{t q}\left(\mu_{t q}, \phi_{t q}\right)\right\} \dot{\ell}_{t q(\lambda)}\left(\mu_{t q}, \phi_{t q}\right), \\
\dot{a}_{t \alpha_{i}(\lambda)} & =\sum_{q=1}^{Q} \frac{\nu_{q}}{\sqrt{\pi}} \exp \left\{\ell_{t q}\left(\mu_{t q}, \phi_{t q}\right)\right\}\left[\dot{\ell}_{t q(\lambda)}\left(\mu_{t q}, \phi_{t q}\right) \dot{\ell}_{t q\left(\alpha_{i}\right)}\left(\mu_{t q}, \phi_{t q}\right)+\ddot{\ell}_{t q\left(\alpha_{i}\right)(\lambda)}\left(\mu_{t q}, \phi_{t q}\right)\right], \\
\ddot{\ell}_{t q\left(\alpha_{i}\right)\left(\gamma_{k}\right)}\left(\mu_{t q}, \phi_{t q}\right) & =\left\{\left(y_{t}^{*}-\mu_{t q}^{*}\right)-c_{t}\right\} \frac{1}{g^{\prime}\left(\mu_{t q}\right)} \frac{1}{h^{\prime}\left(\phi_{t q}\right)} z_{t i} x_{t}^{*},
\end{aligned}
$$

em que $\dot{\ell}_{t q(\lambda)}\left(\mu_{t q}, \phi_{t q}\right)$ é representado em (A.12).

- Para $i=1,2, \ldots, p$ temos que $\frac{\partial^{2} \ell_{a}(\mathbf{\Psi})}{\partial \alpha_{i} \partial \mu_{x}}=J_{a\left(\alpha_{i} \mu_{x}\right)}=\sum_{t=1}^{n}\left\{\dot{b}_{t\left(\mu_{x}\right)} a_{t \alpha_{i}}+b_{t} \dot{a}_{t \alpha_{i}\left(\mu_{x}\right)}\right\}$, em que

$$
\begin{aligned}
\dot{b}_{t\left(\mu_{x}\right)}= & -b_{t}^{-2} \sum_{q=1}^{Q} \frac{\nu_{q}}{\sqrt{\pi}} \exp \left\{\ell_{t q}\left(\mu_{t q}, \phi_{t q}\right)\right\} \dot{\ell}_{t q\left(\mu_{x}\right)}\left(\mu_{t q}, \phi_{t q}\right), \\
\dot{a}_{t \alpha_{i}\left(\mu_{x}\right)=} & \sum_{q=1}^{Q} \frac{\nu_{q}}{\sqrt{\pi}} \exp \left\{\ell_{t q}\left(\mu_{t q}, \phi_{t q}\right)\right\}\left[\dot{\ell}_{t q\left(\mu_{x}\right)}\left(\mu_{t q}, \phi_{t q}\right) \dot{\ell}_{t q\left(\alpha_{i}\right)}\left(\mu_{t q}, \phi_{t q}\right)+\ddot{\ell}_{t q\left(\alpha_{i}\right)\left(\mu_{x}\right)}\left(\mu_{t q}, \phi_{t q}\right)\right], \\
\ddot{\ell}_{t q\left(\alpha_{i}\right)\left(\mu_{x}\right)}\left(\mu_{t q}, \phi_{t q}\right)= & -\phi_{t q}\left\{u_{t}-\left(y_{t}^{*}-\mu_{t q}^{*}\right) \frac{g^{\prime \prime}\left(\mu_{t q}\right)}{\left\{g^{\prime}\left(\mu_{t q}\right)\right\}^{3}}\right\} z_{t i} \beta\left(1-k_{x}\right)+ \\
& \left\{\left(y_{t}^{*}-\mu_{t q}^{*}\right)-c_{t}\right\} \frac{1}{g^{\prime}\left(\mu_{t q}\right)} \frac{1}{h^{\prime}\left(\phi_{t q}\right)} z_{t i} \lambda\left(1-k_{x}\right),
\end{aligned}
$$

em que $\dot{\ell}_{t q\left(\mu_{x}\right)}\left(\mu_{t q}, \phi_{t q}\right)$ é dado em (A.17).

- Para $i=1,2, \ldots, p$ temos que $\frac{\partial^{2} \ell_{a}(\mathbf{\Psi})}{\partial \alpha_{i} \partial \sigma_{x}^{2}}=J_{a\left(\alpha_{i} \sigma_{x}^{2}\right)}=\sum_{t=1}^{n}\left\{\dot{b}_{t\left(\sigma_{x}^{2}\right)} a_{t \alpha_{i}}+b_{t} \dot{a}_{t \alpha_{i}\left(\sigma_{x}^{2}\right)}\right\}$, em que

$$
\begin{aligned}
\dot{b}_{t\left(\sigma_{x}^{2}\right)}= & -b_{t}^{-2} \sum_{q=1}^{Q} \frac{\nu_{q}}{\sqrt{\pi}} \exp \left\{\ell_{t q}\left(\mu_{t q}, \phi_{t q}\right)\right\} \dot{\ell}_{t q\left(\sigma_{x}^{2}\right)}\left(\mu_{t q}, \phi_{t q}\right), \\
\dot{a}_{t \alpha_{i}\left(\sigma_{x}^{2}\right)}= & \sum_{q=1}^{Q} \frac{\nu_{q}}{\sqrt{\pi}} \exp \left\{\ell_{t q}\left(\mu_{t q}, \phi_{t q}\right)\right\}\left[\dot{\ell}_{t q\left(\sigma_{x}^{2}\right)}\left(\mu_{t q}, \phi_{t q}\right) \dot{\ell}_{t q\left(\alpha_{i}\right)}\left(\mu_{t q}, \phi_{t q}\right)+\ddot{\ell}_{t q\left(\alpha_{i}\right)\left(\sigma_{x}^{2}\right)}\left(\mu_{t q}, \phi_{t q}\right)\right], \\
\ddot{\ell}_{t q\left(\alpha_{i}\right)\left(\sigma_{x}^{2}\right)}\left(\mu_{t q}, \phi_{t q}\right)= & -\phi_{t q}\left\{u_{t}-\left(y_{t}^{*}-\mu_{t q}^{*}\right) \frac{g^{\prime \prime}\left(\mu_{t q}\right)}{\left\{g^{\prime}\left(\mu_{t q}\right)\right\}^{3}}\right\} z_{t i} \beta \frac{\left(1-k_{x}\right)^{2}}{2 \sigma_{x_{t} \mid w_{t}}^{2}} s_{q}+ \\
& \left\{\left(y_{t}^{*}-\mu_{t q}^{*}\right)-c_{t}\right\} \frac{1}{g^{\prime}\left(\mu_{t q}\right)} \frac{1}{h^{\prime}\left(\phi_{t q}\right)} z_{t i} \lambda \frac{\left(1-k_{x}\right)^{2}}{2 \sigma_{x_{t} \mid w_{t}}^{2}} s_{q} .
\end{aligned}
$$

em que $\dot{\ell}_{t q\left(\sigma_{x}^{2}\right)}\left(\mu_{t q}, \phi_{t q}\right)$ é dada em (A.18).

- Temos que $\frac{\partial^{2} \ell_{a}(\mathbf{\Psi})}{\partial \beta \partial \beta}=J_{a(\beta \beta)}=\sum_{t=1}^{n}\left\{\dot{b}_{t(\beta)} a_{t \beta}+b_{t} \dot{a}_{t \beta(\beta)}\right\}$, em que 


$$
\begin{aligned}
\dot{a}_{t \beta(\beta)} & =\sum_{q=1}^{Q} \frac{\nu_{q}}{\sqrt{\pi}} \exp \left\{\ell_{t q}\left(\mu_{t q}, \phi_{t q}\right)\right\}\left[\left(\dot{\ell}_{t q(\beta)}\left(\mu_{t q}, \phi_{t q}\right)\right)^{2}+\ddot{\ell}_{t q(\beta)(\beta)}\left(\mu_{t q}, \phi_{t q}\right)\right], \\
\ddot{\ell}_{t q(\beta)(\beta)}\left(\mu_{t q}, \phi_{t q}\right) & =-\phi_{t q}\left\{u_{t}-\left(y_{t}^{*}-\mu_{t q}^{*}\right) \frac{g^{\prime \prime}\left(\mu_{t q}\right)}{\left\{g^{\prime}\left(\mu_{t q}\right)\right\}^{3}}\right\} x_{t}^{* 2} .
\end{aligned}
$$

- Para $k=1,2, \ldots, \breve{q}$ temos que $\frac{\partial^{2} \ell_{a}(\mathbf{\Psi})}{\partial \beta \partial \gamma_{k}}=J_{a\left(\beta \gamma_{k}\right)}=\sum_{t=1}^{n}\left\{\dot{b}_{t\left(\gamma_{k}\right)} a_{t \beta}+b_{t} \dot{a}_{t \beta\left(\gamma_{k}\right)}\right\}$, em que

$$
\begin{aligned}
\dot{a}_{t \beta\left(\gamma_{k}\right)} & =\sum_{q=1}^{Q} \frac{\nu_{q}}{\sqrt{\pi}} \exp \left\{\ell_{t q}\left(\mu_{t q}, \phi_{t q}\right)\right\}\left[\dot{\ell}_{t q\left(\gamma_{k}\right)}\left(\mu_{t q}, \phi_{t q}\right) \dot{\ell}_{t q(\beta)}\left(\mu_{t q}, \phi_{t q}\right)+\ddot{\ell}_{t q(\beta)\left(\gamma_{k}\right)}\left(\mu_{t q}, \phi_{t q}\right)\right], \\
\ddot{\ell}_{t q(\beta)\left(\gamma_{k}\right)}\left(\mu_{t q}, \phi_{t q}\right) & =\left\{\left(y_{t}^{*}-\mu_{t q}^{*}\right)-c_{t}\right\} \frac{1}{g^{\prime}\left(\mu_{t q}\right)} \frac{1}{h^{\prime}\left(\phi_{t q}\right)} x_{t}^{*} v_{t k} .
\end{aligned}
$$

- Agora, $\frac{\partial^{2} \ell_{a}(\mathbf{\Psi})}{\partial \beta \partial \lambda}=J_{a(\beta \lambda)}=\sum_{t=1}^{n}\left\{\dot{b}_{t(\lambda)} a_{t \beta}+b_{t} \dot{a}_{t \beta(\lambda)}\right\}$,

em que

$$
\begin{aligned}
\dot{a}_{t \beta(\lambda)} & =\sum_{q=1}^{Q} \frac{\nu_{q}}{\sqrt{\pi}} \exp \left\{\ell_{t q}\left(\mu_{t q}, \phi_{t q}\right)\right\}\left[\dot{\ell}_{t q(\lambda)}\left(\mu_{t q}, \phi_{t q}\right) \dot{\ell}_{t q(\beta)}\left(\mu_{t q}, \phi_{t q}\right)+\ddot{\ell}_{t q(\beta)(\lambda)}\left(\mu_{t q}, \phi_{t q}\right)\right], \\
\ddot{\ell}_{t q(\beta)\left(\gamma_{k}\right)}\left(\mu_{t q}, \phi_{t q}\right) & =\left\{\left(y_{t}^{*}-\mu_{t q}^{*}\right)-c_{t}\right\} \frac{1}{g^{\prime}\left(\mu_{t q}\right)} \frac{1}{h^{\prime}\left(\phi_{t q}\right)} x_{t}^{* 2} .
\end{aligned}
$$

- Temos $\frac{\partial^{2} \ell_{a}(\mathbf{\Psi})}{\partial \beta \partial \mu_{x}}=J_{a\left(\beta \mu_{x}\right)}=\sum_{t=1}^{n}\left\{\dot{b}_{t\left(\mu_{x}\right)} a_{t \beta}+b_{t} \dot{a}_{t \beta\left(\mu_{x}\right)}\right\}$,

em que

$$
\dot{a}_{t \beta\left(\mu_{x}\right)}=\sum_{q=1}^{Q} \frac{\nu_{q}}{\sqrt{\pi}} \exp \left\{\ell_{t q}\left(\mu_{t q}, \phi_{t q}\right)\right\}\left[\dot{\ell}_{t q\left(\mu_{x}\right)}\left(\mu_{t q}, \phi_{t q}\right) \dot{\ell}_{t q(\beta)}\left(\mu_{t q}, \phi_{t q}\right)+\ddot{\ell}_{t q(\beta)\left(\mu_{x}\right)}\left(\mu_{t q}, \phi_{t q}\right)\right],
$$

$\ddot{\ell}_{t q(\beta)\left(\mu_{x}\right)}\left(\mu_{t q}, \phi_{t q}\right)=-\beta\left(1-k_{x}\right) \phi_{t q}\left\{u_{t}-\left(y_{t}^{*}-\mu_{t q}^{*}\right) \frac{g^{\prime \prime}\left(\mu_{t q}\right)}{\left\{g^{\prime}\left(\mu_{t q}\right)\right\}^{3}}\right\} x_{t}^{*}+$

$$
\lambda\left(1-k_{x}\right)\left\{\left(y_{t}^{*}-\mu_{t q}^{*}\right)-c_{t}\right\} \frac{1}{g^{\prime}\left(\mu_{t q}\right)} \frac{1}{h^{\prime}\left(\phi_{t q}\right)} x_{t}^{*} .
$$

- Temos também que $\frac{\partial^{2} \ell_{a}(\mathbf{\Psi})}{\partial \beta \partial \sigma_{x}^{2}}=J_{a\left(\beta \sigma_{x}^{2}\right)}=\sum_{t=1}^{n}\left\{\dot{b}_{t\left(\sigma_{x}^{2}\right)} a_{t \beta}+b_{t} \dot{a}_{t \beta\left(\sigma_{x}^{2}\right)}\right\}$,

em que

$$
\begin{aligned}
\dot{a}_{t \beta\left(\sigma_{x}^{2}\right)=} & \sum_{q=1}^{Q} \frac{\nu_{q}}{\sqrt{\pi}} \exp \left\{\ell_{t q}\left(\mu_{t q}, \phi_{t q}\right)\right\}\left[\dot{\ell}_{t q\left(\sigma_{x}^{2}\right)}\left(\mu_{t q}, \phi_{t q}\right) \dot{\ell}_{t q(\beta)}\left(\mu_{t q}, \phi_{t q}\right)+\ddot{\ell}_{t q(\beta)\left(\sigma_{x}^{2}\right)}\left(\mu_{t q}, \phi_{t q}\right)\right], \\
\ddot{\ell}_{t q(\beta)\left(\mu_{x}\right)}\left(\mu_{t q}, \phi_{t q}\right)= & -\beta \frac{\left(1-k_{x}\right)}{2 \sigma_{x}^{2}} \phi_{t q}\left\{u_{t}-\left(y_{t}^{*}-\mu_{t q}^{*}\right) \frac{g^{\prime \prime}\left(\mu_{t q}\right)}{\left\{g^{\prime}\left(\mu_{t q}\right)\right\}^{3}}\right\} x_{t}^{*} s_{q}+ \\
& \lambda \frac{\left(1-k_{x}\right)}{2 \sigma_{x}^{2}}\left\{\left(y_{t}^{*}-\mu_{t q}^{*}\right)-c_{t}\right\} \frac{1}{g^{\prime}\left(\mu_{t q}\right)} \frac{1}{h^{\prime}\left(\phi_{t q}\right)} x_{t}^{*} s_{q} .
\end{aligned}
$$

- Para $k, j=1,2, \ldots, \breve{q}$ temos que $\frac{\partial^{2} \ell_{a}(\boldsymbol{\Psi})}{\partial \gamma_{k} \partial \gamma_{j}}=J_{a\left(\gamma_{k} \gamma_{j}\right)}=\sum_{t=1}^{n}\left\{\dot{b}_{t\left(\gamma_{j}\right)} a_{t \gamma_{k}}+b_{t} \dot{a}_{t \gamma_{k}\left(\gamma_{j}\right)}\right\}$, em que 


$$
\dot{a}_{t \gamma_{k}\left(\gamma_{j}\right)}=\sum_{q=1}^{Q} \frac{\nu_{q}}{\sqrt{\pi}} \exp \left\{\ell_{t q}\left(\mu_{t q}, \phi_{t q}\right)\right\}\left[\dot{\ell}_{t q\left(\gamma_{j}\right)}\left(\mu_{t q}, \phi_{t q}\right) \dot{\ell}_{t q\left(\gamma_{k}\right)}\left(\mu_{t q}, \phi_{t q}\right)+\ddot{\ell}_{t q\left(\gamma_{k} \gamma_{j}\right)}\left(\mu_{t q}, \phi_{t q}\right)\right] \text {, }
$$

$\ddot{\ell}_{t q\left(\gamma_{k} \gamma_{j}\right)}\left(\mu_{t q}, \phi_{t q}\right)=-v_{t k}\left\{d_{t}+a_{t} \frac{h^{\prime \prime}\left(\phi_{t q}\right)}{\left\{h^{\prime}\left(\phi_{t q}\right)\right\}^{3}}\right\} v_{t j}$.

- Para $k=1,2, \ldots, \breve{q}$ temos que $\frac{\partial^{2} \ell_{a}(\mathbf{\Psi})}{\partial \gamma_{k} \partial \lambda}=J_{a\left(\gamma_{k} \lambda\right)}=\sum_{t=1}^{n}\left\{\dot{b}_{t(\lambda)} a_{t \gamma_{k}}+b_{t} \dot{a}_{t \gamma_{k}(\lambda)}\right\}$,

em que

$$
\begin{aligned}
\dot{a}_{t \gamma_{k}(\lambda)} & =\sum_{q=1}^{Q} \frac{\nu_{q}}{\sqrt{\pi}} \exp \left\{\ell_{t q}\left(\mu_{t q}, \phi_{t q}\right)\right\}\left[\dot{\ell}_{t q(\lambda)}\left(\mu_{t q}, \phi_{t q}\right) \dot{\ell}_{t q\left(\gamma_{k}\right)}\left(\mu_{t q}, \phi_{t q}\right)+\ddot{\ell}_{t q\left(\gamma_{k} \lambda\right)}\left(\mu_{t q}, \phi_{t q}\right)\right], \\
\ddot{\ell}_{t q\left(\gamma_{k} \lambda\right)}\left(\mu_{t q}, \phi_{t q}\right) & =-v_{t k}\left\{d_{t}+a_{t} \frac{h^{\prime \prime}\left(\phi_{t q}\right)}{\left\{h^{\prime}\left(\phi_{t q}\right)\right\}^{3}}\right\} x_{t}^{*} .
\end{aligned}
$$

- Para $k=1,2, \ldots, \breve{q}$ temos que $\frac{\partial^{2} \ell_{a}(\boldsymbol{\Psi})}{\partial \gamma_{k} d \mu_{x}}=J_{a\left(\gamma_{k} \mu_{x}\right)}=\sum_{t=1}^{n}\left\{\dot{b}_{t\left(\mu_{x}\right)} a_{t \gamma_{k}}+b_{t} \dot{a}_{t \gamma_{k}\left(\mu_{x}\right)}\right\}$, em que

$$
\begin{aligned}
\dot{a}_{t \gamma_{k}\left(\mu_{x}\right)=} & \sum_{q=1}^{Q} \frac{\nu_{q}}{\sqrt{\pi}} \exp \left\{\ell_{t q}\left(\mu_{t q}, \phi_{t q}\right)\right\}\left[\dot{\ell}_{t q\left(\mu_{x}\right)}\left(\mu_{t q}, \phi_{t q}\right) \dot{\ell}_{t q\left(\gamma_{k}\right)}\left(\mu_{t q}, \phi_{t q}\right)+\ddot{\ell}_{t q\left(\gamma_{k} \mu_{x}\right)}\left(\mu_{t q}, \phi_{t q}\right)\right], \\
\ddot{\ell}_{t q\left(\gamma_{k} \mu_{x}\right)}\left(\mu_{t q}, \phi_{t q}\right)= & -\beta\left(1-k_{x}\right) \phi_{t q}\left\{u_{t}-\left(y_{t}^{*}-\mu_{t q}^{*}\right) \frac{g^{\prime \prime}\left(\mu_{t q}\right)}{\left\{g^{\prime}\left(\mu_{t q}\right)\right\}^{3}}\right\} v_{t k}+ \\
& \lambda\left(1-k_{x}\right)\left\{\left(y_{t}^{*}-\mu_{t q}^{*}\right)-c_{t}\right\} \frac{1}{g^{\prime}\left(\mu_{t q}\right)} \frac{1}{h^{\prime}\left(\phi_{t q}\right)} v_{t k} .
\end{aligned}
$$

- Para $k=1,2, \ldots, \breve{q}$ temos que $\frac{\partial^{2} \ell_{a}(\boldsymbol{\Psi})}{\partial \gamma_{k} \partial \sigma_{x}^{2}}=J_{a\left(\gamma_{k} \sigma_{x}^{2}\right)}=\sum_{t=1}^{n}\left\{\dot{b}_{t\left(\sigma_{x}^{2}\right)} a_{t \gamma_{k}}+b_{t} \dot{a}_{t \gamma_{k}\left(\sigma_{x}^{2}\right)}\right\}$, em que

$$
\begin{aligned}
\dot{a}_{t \gamma_{k}\left(\sigma_{x}^{2}\right)=} & \sum_{q=1}^{Q} \frac{\nu_{q}}{\sqrt{\pi}} \exp \left\{\ell_{t q}\left(\mu_{t q}, \phi_{t q}\right)\right\}\left[\dot{\ell}_{t q\left(\sigma_{x}^{2}\right)}\left(\mu_{t q}, \phi_{t q}\right) \dot{\ell}_{t q\left(\gamma_{k}\right)}\left(\mu_{t q}, \phi_{t q}\right)+\ddot{\ell}_{t q\left(\gamma_{k} \sigma_{x}^{2}\right)}\left(\mu_{t q}, \phi_{t q}\right)\right], \\
\ddot{\ell}_{t q\left(\gamma_{k} \sigma_{x}^{2}\right)}\left(\mu_{t q}, \phi_{t q}\right)= & -\beta \frac{\left(1-k_{x}\right)}{2 \sigma_{x}^{2}} \phi_{t q}\left\{u_{t}-\left(y_{t}^{*}-\mu_{t q}^{*}\right) \frac{g^{\prime \prime}\left(\mu_{t q}\right)}{\left\{g^{\prime}\left(\mu_{t q}\right)\right\}^{3}}\right\} v_{t k} s_{q}+ \\
& \lambda \frac{\left(1-k_{x}\right)}{2 \sigma_{x}^{2}}\left\{\left(y_{t}^{*}-\mu_{t q}^{*}\right)-c_{t}\right\} \frac{1}{g^{\prime}\left(\mu_{t q}\right)} \frac{1}{h^{\prime}\left(\phi_{t q}\right)} v_{t k} s_{q} .
\end{aligned}
$$

- Temos que $\frac{\partial^{2} \ell_{a}(\mathbf{\Psi})}{\partial \lambda \partial \lambda}=J_{a(\lambda \lambda)}=\sum_{t=1}^{n}\left\{\dot{b}_{t(\lambda)} a_{t \lambda}+b_{t} \dot{a}_{t \lambda(\lambda)}\right\}$, em que

$$
\begin{aligned}
\dot{a}_{t \lambda(\lambda)} & =\sum_{q=1}^{Q} \frac{\nu_{q}}{\sqrt{\pi}} \exp \left\{\ell_{t q}\left(\mu_{t q}, \phi_{t q}\right)\right\}\left[\left(\dot{\ell}_{t q(\lambda)}\left(\mu_{t q}, \phi_{t q}\right)\right)^{2}+\ddot{\ell}_{t q(\lambda \lambda)}\left(\mu_{t q}, \phi_{t q}\right)\right], \\
\ddot{\ell}_{t q(\lambda \lambda)}\left(\mu_{t q}, \phi_{t q}\right) & =-x_{t}^{*}\left\{d_{t}+a_{t} \frac{h^{\prime \prime}\left(\phi_{t q}\right)}{\left\{h^{\prime}\left(\phi_{t q}\right)\right\}^{3}}\right\} x_{t}^{*} .
\end{aligned}
$$

- Temos que $\frac{\partial^{2} \ell_{a}(\boldsymbol{\Psi})}{\partial \lambda \partial \mu_{x}}=J_{a\left(\lambda \mu_{x}\right)}=\sum_{t=1}^{n}\left\{\dot{b}_{t\left(\mu_{x}\right)} a_{t \lambda}+b_{t} \dot{a}_{t \lambda\left(\mu_{x}\right)}\right\}$, em que 
94

APÊNDICE A

$$
\begin{aligned}
\dot{a}_{t \lambda\left(\mu_{x}\right)}= & \sum_{q=1}^{Q} \frac{\nu_{q}}{\sqrt{\pi}} \exp \left\{\ell_{t q}\left(\mu_{t q}, \phi_{t q}\right)\right\}\left[\dot{\ell}_{t q\left(\mu_{x}\right)}\left(\mu_{t q}, \phi_{t q}\right) \dot{\ell}_{t q(\lambda)}\left(\mu_{t q}, \phi_{t q}\right)+\ddot{\ell}_{t q\left(\lambda \mu_{x}\right)}\left(\mu_{t q}, \phi_{t q}\right)\right], \\
\ddot{\ell}_{t q\left(\lambda \mu_{x}\right)}\left(\mu_{t q}, \phi_{t q}\right)= & \frac{x_{t q}^{*}}{h^{\prime}\left(\phi_{t q}\right)}\left[\dot{a}_{t q\left(\mu_{x}\right)}-\lambda\left(1-k_{x}\right) a_{t q} \frac{h^{\prime \prime}\left(\phi_{t q}\right)}{\left\{h^{\prime}\left(\phi_{t q}\right)\right\}^{2}}\right]+\frac{\left(1-k_{x}\right) a_{t q}}{h^{\prime}\left(\phi_{t q}\right)}, \\
\dot{a}_{t q\left(\mu_{x}\right)}= & \beta\left(1-k_{x}\right) \frac{\left(y_{t}^{*}-\mu_{T}^{*}\right)}{g^{\prime}\left(\mu_{t q}\right)}-\mu_{t q}\left(1-k_{x}\right) \psi^{\prime}\left(\mu_{t q} \phi_{t q}\right)\left[\beta \frac{\phi_{t q}}{g^{\prime}\left(\mu_{t q}\right)}+\lambda \frac{\mu_{t q}}{h^{\prime}\left(\phi_{t q}\right)}\right]- \\
& \left(1-k_{x}\right)\left(1-\mu_{t q}\right) \psi^{\prime}\left[\left(1-\mu_{t q}\right) \phi_{t q}\right]\left\{\lambda \frac{1-\mu_{t q}}{h^{\prime}\left(\phi_{t q}\right)}-\beta \frac{\phi_{t q}}{g^{\prime}\left(\mu_{t q}\right)}\right\}+\lambda \psi^{\prime}\left(\phi_{t q}\right) \frac{1-k_{x}}{h^{\prime}\left(\phi_{t q}\right)}
\end{aligned}
$$

- Memos que $\frac{\partial^{2} \ell_{a}(\mathbf{\Psi})}{\partial \lambda \partial \sigma_{x}^{2}}=J_{a\left(\lambda \sigma_{x}^{2}\right)}=\sum_{t=1}^{n}\left\{\dot{b}_{t\left(\mu_{x}\right)} a_{t \lambda}+b_{t} \dot{a}_{t \lambda\left(\sigma_{x}^{2}\right)}\right\}$, em que

$$
\begin{aligned}
& \dot{a}_{t \lambda\left(\sigma_{x}^{2}\right)}=\sum_{q=1}^{Q} \frac{\nu_{q}}{\sqrt{\pi}} \exp \left\{\ell_{t q}\left(\mu_{t q}, \phi_{t q}\right)\right\}\left[\dot{\ell}_{t q\left(\sigma_{x}^{2}\right)}\left(\mu_{t q}, \phi_{t q}\right) \dot{\ell}_{t q(\lambda)}\left(\mu_{t q}, \phi_{t q}\right)+\ddot{\ell}_{t q\left(\lambda \sigma_{x}^{2}\right)}\left(\mu_{t q}, \phi_{t q}\right)\right], \\
& \ddot{\ell}_{t q\left(\lambda \sigma_{x}^{2}\right)}\left(\mu_{t q}, \phi_{t q}\right)=\frac{\dot{a}_{t q\left(\sigma_{x}^{2}\right)} x_{t q}^{*}}{h^{\prime}\left(\phi_{t q}\right)}-\frac{\left(1-k_{x}\right)^{2} a_{t q}}{h^{\prime}\left(\phi_{t q}\right)}\left[\frac{w_{t}-\mu_{x}}{\sigma_{e}^{2}}+\frac{s_{q}}{\sqrt{2 \sigma_{x_{t} \mid w_{t}}^{2}}}\right]\left[\frac{x_{t q}^{*} h^{\prime \prime}\left(\phi_{t q}\right)}{\left\{h^{\prime}\left(\phi_{t q}\right)\right\}^{2}}-1\right] \text {, } \\
& \dot{a}_{t q\left(\sigma_{x}^{2}\right)}=\left(y_{t}^{*}-\mu_{t}^{*}\right) \dot{\mu}_{t q\left(\sigma_{x}^{2}\right)}-\mu_{t q} \dot{\mu}_{t q\left(\sigma_{x}^{2}\right)}^{*}-\left[\left(1-\mu_{t q}\right) \dot{\phi}_{t q\left(\sigma_{x}^{2}\right)}-\phi_{t q} \dot{\mu}_{t q\left(\sigma_{x}^{2}\right)}\right] \psi^{\prime}\left[\left(1-\mu_{t q}\right) \phi_{t q}\right] \text {, } \\
& \dot{\mu}_{t q\left(\sigma_{x}^{2}\right)}^{*}=\left\{\mu_{t q} \dot{\phi}_{t q\left(\sigma_{x}^{2}\right)}+\phi_{t q} \dot{\mu}_{t q\left(\sigma_{x}^{2}\right)}\right\} \psi^{\prime}\left(\mu_{t q}, \phi_{t q}\right)+ \\
& \left\{\left(1-\mu_{t q}\right) \dot{\phi}_{t q\left(\sigma_{x}^{2}\right)}-\phi_{t q} \dot{\mu}_{t q\left(\sigma_{x}^{2}\right)}\right\} \psi^{\prime}\left[\left(1-\mu_{t q}\right) \phi_{t q}\right], \\
& \dot{\mu}_{t q\left(\sigma_{x}^{2}\right)}=\frac{\beta\left(1-k_{x}\right)^{2}}{g^{\prime}\left(\mu_{t q}\right)}\left[\frac{w_{t}-\mu_{x}}{\sigma_{2}^{2}}+\frac{s_{q}}{\sqrt{2 \sigma_{x_{t} \mid w_{t}}^{2}}}\right] \text {, } \\
& \dot{\phi}_{t q\left(\sigma_{x}^{2}\right)}=\frac{\lambda\left(1-k_{x}\right)^{2}}{h^{\prime}\left(\phi_{t q}\right)}\left[\frac{w_{t}-\mu_{x}}{\sigma_{2}^{2}}+\frac{s_{q}}{\sqrt{2 \sigma_{x_{t} \mid w_{t}}^{2}}}\right] \text {. }
\end{aligned}
$$

em que

$$
\begin{aligned}
\dot{a}_{t \mu_{x}\left(\mu_{x}\right)=} & \sum_{q=1}^{Q} \frac{\nu_{q}}{\sqrt{\pi}} \exp \left\{\ell_{t q}\left(\mu_{t q}, \phi_{t q}\right)\right\}\left[\left(\dot{\ell}_{t q\left(\mu_{x}\right)}\left(\mu_{t q}, \phi_{t q}\right)\right)^{2}+\ddot{\ell}_{t q\left(\mu_{x} \mu_{x}\right)}\left(\mu_{t q}, \phi_{t q}\right)\right], \\
\ddot{\ell}_{t q\left(\mu_{x} \mu_{x}\right)}\left(\mu_{t q}, \phi_{t q}\right)= & \frac{\beta\left(1-k_{x}\right)}{\left\{g^{\prime}\left(\mu_{t q}\right)\right\}^{2}}\left[g^{\prime}\left(\mu_{t q}\right) p_{t q}+\beta\left(1-k_{x}\right) \phi_{t q}\left(y_{t}^{*}-\mu_{t q}\right)+\frac{g^{\prime \prime}\left(\mu_{t q}\right)}{g^{\prime}\left(\mu_{t q}\right)}\right]+ \\
& \frac{\lambda\left(1-k_{x}\right)}{\left\{h^{\prime}\left(\phi_{t q}\right)\right\}^{2}}\left[h^{\prime}\left(\phi_{t q}\right) \dot{a}_{t\left(\mu_{x}\right)}+\lambda\left(1-k_{x}\right) a_{t} \frac{h^{\prime \prime}\left(\phi_{t q}\right)}{h^{\prime}\left(\phi_{t q}\right)}\right], \\
p_{t q}= & \left(y_{t}^{*}-\mu_{t}^{*}\right) \dot{\phi}_{t q\left(\mu_{x}\right)}-\phi_{t q} \dot{\mu}_{t q(\mu)}^{*}, \\
\dot{\mu}_{t q\left(\mu_{x}\right)}^{*}= & \left\{\mu_{t q} \dot{\phi}_{t q\left(\mu_{x}\right)}+\phi_{t q} \dot{\mu}_{t q\left(\mu_{x}\right)}\right\} \psi^{\prime}\left(\mu_{t q}, \phi_{t q}\right)+ \\
& \left\{\left(1-\mu_{t q}\right) \dot{\phi}_{t q\left(\mu_{x}\right)}-\phi_{t q} \dot{\mu}_{t q\left(\mu_{x}\right)}\right\} \psi^{\prime}\left[\left(1-\mu_{t q}\right) \phi_{t q}\right],
\end{aligned}
$$




$$
\begin{aligned}
& \dot{\mu}_{t q\left(\mu_{x}\right)}=\frac{\beta\left(1-k_{x}\right)}{g^{\prime}\left(\mu_{t q}\right)}, \\
& \dot{\phi}_{t q\left(\mu_{x}\right)}=\frac{\lambda\left(1-k_{x}\right)}{h^{\prime}\left(\phi_{t q}\right)} .
\end{aligned}
$$

- Temos que $\frac{\partial^{2} \ell_{a}(\boldsymbol{\Psi})}{\partial \mu_{x} \partial \sigma_{x}^{2}}=J_{a\left(\mu_{x} \sigma_{x}^{2}\right)}=-\frac{1}{\left(\sigma_{x}^{2}+\sigma_{e}^{2}\right)} \sum_{t=1}^{n}\left(w_{t}-\mu_{x}\right)+\sum_{t=1}^{n}\left\{\dot{b}_{t\left(\sigma_{x}^{2}\right)} a_{t \mu_{x}}+b_{t} \dot{a}_{t \mu_{x}\left(\sigma_{x}^{2}\right)}\right\}$, em que

$$
\begin{aligned}
\dot{a}_{t \mu_{x}\left(\sigma_{x}^{2}\right)} & =\sum_{q=1}^{Q} \frac{\nu_{q}}{\sqrt{\pi}} \exp \left\{\ell_{t q}\left(\mu_{t q}, \phi_{t q}\right)\right\}\left[\dot{\ell}_{t q\left(\sigma_{x}^{2}\right)}\left(\mu_{t q}, \phi_{t q}\right) \dot{\ell}_{t q\left(\mu_{x}\right)}\left(\mu_{t q}, \phi_{t q}\right)+\ddot{\ell}_{t q\left(\mu_{x} \sigma_{x}^{2}\right)}\left(\mu_{t q}, \phi_{t q}\right)\right], \\
\ddot{\ell}_{t q\left(\mu_{x} \sigma_{x}^{2}\right)}\left(\mu_{t q}, \phi_{t q}\right) & =-\frac{1-k_{x}}{\sigma_{e}^{2}} \dot{\ell}_{t q\left(\mu_{x}\right)}\left(\mu_{t q}, \phi_{t q}\right)+\left(1-k_{x}\right)\left[\beta \frac{m_{t q}}{\left\{g^{\prime}\left(\mu_{t q}\right)\right\}^{2}}+\lambda \frac{n_{t q}}{\left\{h^{\prime}\left(\phi_{t q}\right)\right\}^{2}}\right], \\
m_{t q} & =\phi_{t q}\left(y_{t}^{*}-\mu_{t q}\right) g^{\prime \prime}\left(\mu_{t q}\right) \dot{\mu}_{t q\left(\sigma_{x}^{2}\right)}+g^{\prime}\left(\mu_{t q}\right)\left[\left(y_{t}^{*}-\mu_{t q}^{*}\right) \dot{\phi}_{t q\left(\sigma_{x}^{2}\right)}-\phi_{t q} \dot{\mu}_{t q\left(\sigma_{x}^{2}\right)}^{*}\right], \\
n_{t q} & =\dot{a}_{t\left(\sigma_{x}^{2}\right)} h^{\prime}\left(\phi_{t q}\right)+a_{t} h^{\prime \prime}\left(\phi_{t q}\right) \dot{\phi}_{t q\left(\sigma_{x}^{2}\right)} .
\end{aligned}
$$

- Temos que

$$
\begin{aligned}
\frac{\partial^{2} \ell_{a}(\mathbf{\Psi})}{\partial \sigma_{x}^{2} \partial \sigma_{x}^{2}}=J_{a\left(\sigma_{x}^{2} \sigma_{x}^{2}\right)=} & -\sum_{t=1}^{n}\left\{-\frac{1}{2\left(\sigma_{x}^{2}+\sigma_{e}^{2}\right)^{2}}\left[1-\frac{\left(w_{t}-\mu_{t}\right)^{2}}{\sigma_{x}^{2}+\sigma_{e}^{2}}\right]+\frac{1}{2\left(\sigma_{x}^{2}+\sigma_{e}^{2}\right)}\left[\frac{w_{t}-\mu_{x}}{\sigma_{x}^{2}+\sigma_{e}^{2}}\right]^{2}\right\}+ \\
& \sum_{t=1}^{n}\left\{\dot{b}_{t\left(\sigma_{x}^{2}\right)} a_{t \sigma_{x}^{2}}+b_{t} \dot{a}_{t \sigma_{x}^{2}\left(\sigma_{x}^{2}\right)}\right\},
\end{aligned}
$$

em que

$$
\begin{aligned}
\dot{a}_{t \sigma_{x}^{2}\left(\sigma_{x}^{2}\right)} & =\sum_{q=1}^{Q} \frac{\nu_{q}}{\sqrt{\pi}} \exp \left\{\ell_{t q}\left(\mu_{t q}, \phi_{t q}\right)\right\}\left[\left(\dot{\ell}_{t q\left(\sigma_{x}^{2}\right)}\left(\mu_{t q}, \phi_{t q}\right)\right)^{2}+\ddot{\ell}_{t q\left(\sigma_{x}^{2} \sigma_{x}^{2}\right)}\left(\mu_{t q}, \phi_{t q}\right)\right], \\
\ddot{\ell}_{t q\left(\sigma_{x}^{2} \sigma_{x}^{2}\right)}\left(\mu_{t q}, \phi_{t q}\right) & =-\left(\frac{1-k_{x}}{2 \sigma_{x_{t} \mid w_{t}}^{2}}\right)^{2}\left[\frac{\sigma_{x_{t} \mid w_{t}}^{2}}{\sigma_{e}^{2}+\left(1-k_{x}\right)}\right] \dot{\ell}_{t q\left(\mu_{x}\right)}\left(\mu_{t q}, \phi_{t q}\right) s_{q}+\frac{1-k_{x}}{2 \sigma_{x_{t} \mid w_{t}}^{2}} \ddot{\ell}_{t q\left(\mu_{x} \sigma_{x}^{2}\right)\left(\mu_{t q}, \phi_{t q}\right)} s_{q} .
\end{aligned}
$$

\section{A.3 Vetor escore e matriz de informação observada restrita}

\section{A.3.1 Vetor escore}

Apresentamos neste apêndice as primeiras derivadas de $\ell_{r}(\boldsymbol{\xi})$ em relação aos parâmetros de $\boldsymbol{\xi}$, ou seja a função escore. O logaritmo da função de verossimilhança restrita é dada por

$$
\ell_{r}(\boldsymbol{\xi} ; w)=\sum_{t=1}^{n} \log f\left(w_{t} ; \boldsymbol{\xi}, \sigma_{e}^{2}\right),
$$

sendo $\sigma_{e}^{2}$ conhecido ou estimável. Assumimos em (2.2) que a covariável não observada $x_{t}$ é normalmente distribuída com media $\mu_{x}$ e variância $\sigma_{x}^{2}$, conseqüentemente desse fato é que $w_{t} \sin N\left(\mu_{x} ; \sigma_{x}^{2}+\right.$ $\left.\sigma_{e}^{2}\right)$. Assim, o logaritmo da função de verossimilhança restrita agora é dada por

$$
\ell_{r}(\boldsymbol{\xi} ; w)=-\frac{n}{2} \log \left[2 \pi\left(\sigma_{x}^{2}+\sigma_{e}^{2}\right)\right]-\frac{1}{2\left(\sigma_{x}^{2}+\sigma_{e}^{2}\right)} \sum_{t=1}^{n}\left(w_{t}-\mu_{x}\right)^{2} .
$$


Então,

$$
\begin{aligned}
\frac{\partial \ell_{r}(\boldsymbol{\xi} ; w)}{\partial \mu_{x}} & =\frac{1}{\sigma_{x}^{2}+\sigma_{e}^{2}} \sum_{t=1}^{n}\left(w_{t}-\mu_{x}\right), \\
\frac{\partial \ell_{r}(\boldsymbol{\xi} ; w)}{\partial \sigma_{x}^{2}} & =-\frac{n}{2\left(\sigma_{x}^{2}+\sigma_{e}^{2}\right)}+\frac{1}{2\left(\sigma_{x}^{2}+\sigma_{e}^{2}\right)^{2}} \sum_{t=1}^{n}\left(w_{t}-\mu_{x}\right)^{2} .
\end{aligned}
$$

\section{A.3.2 Matriz de informação observada}

Determinamos nesta sub seção as segundas derivadas do logaritmo da função de verossimilhança restrita. Então,

$$
\begin{aligned}
\frac{\partial^{2} \ell_{r}(\boldsymbol{\xi} ; w)}{\partial \mu_{x}^{2}} & =-\frac{1}{2\left(\sigma_{x}^{2}+\sigma_{e}^{2}\right)} \\
\frac{\partial^{2} \ell_{r}(\boldsymbol{\xi} ; w)}{\partial \mu_{x} \sigma_{x}^{2}} & =0 \\
\frac{\partial^{2} \ell_{r}(\boldsymbol{\xi} ; w)}{\partial\left(\sigma_{x}^{2}\right)^{2}} & =\frac{n}{2\left(\sigma_{x}^{2}+\sigma_{e}^{2}\right)^{2}}-\frac{1}{2\left(\sigma_{x}^{2}+\sigma_{e}^{2}\right)^{3}} \sum_{t=1}^{n}\left(w_{t}-\mu_{x}\right)^{2} .
\end{aligned}
$$

\section{A.4 Método Bootstrap não paramétrico}

A técnica bootstrap tem um importante papel na análise estatística, em especial, quando o cálculo dos estimadores por métodos analíticos é complicado. Esta técnica foi proposta por Efron (1979), tendo grande utilidade nos últimos anos. A ideia central desta proposta é reamostrar as observações de forma adequada, sorteando réplicas da amostra original, para com elas estimar o viés, o erro padrão, calcular intervalos de confiança e testar hipóteses. A técnica tem por base a ideia de que o pesquisador pode tratar sua amostra como se fosse a população que deu origem aos dados e usar amostragem com reposição da amostra original para gerar pseudo-amostras. A partir destas pseudo-amostras, é possível estimar características da população, tais como média, variância, percentis, etc. Uma referência importante sobre a técnica bootstrap é Efron e Tibshirani (1994).

Existem três abordagens da metodologia bootstrap na literatura, a saber: paramétrico, não paramétrico e bayesiano. A diferença entre eles é a forma como são geradas amostras na análise em estudo. Neste trabalho, nos concentramos em utilizar o método não paramétrico. A técnica bootstrap não paramétrico consiste em selecionar $\mathrm{B}$ amostras independentes de tamanho $n$, com reposição, a partir dos dados observados. Suponhamos $\widehat{\boldsymbol{\Psi}}$ e $\widehat{\boldsymbol{\Psi}}^{(b)}$ vetores de estimativas de $\boldsymbol{\Psi}$, sendo que $\widehat{\boldsymbol{\Psi}}^{(b)}$ é obtido a partir da $b$-ésima amostra bootstrap gerada, $b=1, \ldots, B$.

Definamos $\overline{\mathbf{\Psi}}=B^{-1} \sum_{b=1}^{B} \widehat{\mathbf{\Psi}}^{(b)}$, da forma que a matriz de variâncias e covariâncias de $\widehat{\Psi}$ pode ser estimada, por

$$
\widehat{\operatorname{Var}}(\widehat{\mathbf{\Psi}})=\frac{1}{B-1} \sum_{b=1}^{B}\left(\widehat{\mathbf{\Psi}}^{(b)}-\overline{\mathbf{\Psi}}\right)\left(\widehat{\mathbf{\Psi}}^{(b)}-\overline{\mathbf{\Psi}}\right)^{\top}
$$

Portanto, o erro padrão de $\widehat{\boldsymbol{\Psi}}$ é estimado pelo erro padrão amostral das $B$ réplicas, isto é

$$
e p(\widehat{\Psi})=\sqrt{\widehat{\operatorname{Var}}(\widehat{\Psi})} .
$$

Efron e Tibshirani (1994, Cap. 6) sugerem que o número de réplicas bootstrap para estimar o erro padrão esteja no intervalo [25; 200]. Através da metodologia bootstrap, é possível calcular intervalos de confiança que apresentem níveis de cobertura próximos da verdadeira probabilidade de cobertura nominal. Há vários intervalos bootstrap, como por exemplo, intervalo bootstrap percentil, Intervalo bootstrap-t, intervalo bootstrap BC (Bias-Corrected) e intervalo bootstrap $\mathrm{BCa}$ (bias-corrected 
and accelerated). Para descrições detalhas do cálculo de intervalos de confiança bootstrap, ver (Efron e Tibshirani, 1994, Seção 14.3). Nesta tese, restringimo-nos a encontrar unicamente o intervalo bootstrap $\mathrm{BCa}$, apresentando a seguir os passos para calcular os limites inferior e superior do intervalo:

- Inicialmente geramos B amostras bootstrap com reposição;

- para cada amostra bootstrap gerada, calculamos $\widehat{\Psi}^{(b)}$, para $b=1,2, \ldots, B$;

- suponhamos $\widehat{\Psi}=\left(\widehat{\Psi}_{1}, \ldots, \widehat{\Psi}_{j}, \ldots, \widehat{\Psi}_{p}\right)^{\top}$ é o vetor de estimativas de $\boldsymbol{\Psi}$. Ordenamos a $j$-ésima estimativa em ordem crescente,

$$
\widehat{\Psi}_{j}^{(1)} \leq \widehat{\Psi}_{j}^{(2)} \leq \ldots \leq \widehat{\Psi}_{j}^{(B)}, \quad \text { para } \quad j=1, \ldots, p
$$

- o intervalo bootstrap BCa para $\Psi_{j}$, com nível de cobertura de aproximadamente $100(1-2 \alpha) \%$ é dado por $\left(\widehat{\Psi}_{j}^{\left(\alpha_{1}\right)} ; \widehat{\Psi}_{j}^{\left(\alpha_{2}\right)}\right)$, em que os percentis $\alpha_{1}$ e $\alpha_{2}$ são dados por

$$
\begin{aligned}
\alpha_{1} & =\Phi\left(\widehat{z}_{0}+\frac{\widehat{z}_{0}+z^{\alpha}}{1-\widehat{a}\left(\widehat{z}_{0}+z^{\alpha}\right)}\right), \\
\alpha_{2} & =\Phi\left(\widehat{z}_{0}+\frac{\widehat{z}_{0}+z^{1-\alpha}}{1-\widehat{a}\left(\widehat{z}_{0}+z^{1-\alpha}\right)}\right),
\end{aligned}
$$

sendo que $\Phi(\cdot)$ é a função de distribuição normal padrão, $z^{\alpha}$ é o percentil de ordem $100 \alpha \%$ da distribuição normal padrão e a constante $\widehat{a}$ que mede a razão de mudança do erro padrão de $\widehat{\Psi}_{j}$ com respeito ao verdadeiro valor do parâmetro, é dado por

$$
\widehat{a}=\frac{\sum_{t=1}^{n}\left(\widehat{\Psi}_{j(\cdot)}-\widehat{\Psi}_{j(t)}\right)^{3}}{6\left\{\sum_{t=1}^{n}\left(\widehat{\Psi}_{j(\cdot)}-\widehat{\Psi}_{j(t)}\right)^{2}\right\}^{3 / 2}},
$$

em que $\widehat{\Psi}_{j(\cdot)}=n^{-1} \sum_{t=1}^{n} \widehat{\Psi}_{j(t)}, \widehat{\Psi}_{j(t)}$ é estimativa de $\Psi_{j}$ com a $t$-ésima observação eliminada. $\mathrm{O}$ valor de correção do viés $\widehat{z}_{0}$ é obtido diretamente da proporção de réplicas bootstrap menores da estimativa original $\Psi$, isto é

$$
\widehat{z}_{0}=\Phi^{-1}\left(\frac{\sharp\left\{\widehat{\Psi}_{j}^{(b)}<\widehat{\Psi}_{j}\right\}}{B}\right),
$$

para $b=1, \ldots, B$ e $\Phi^{-1}(\cdot)$ indicando a função inversa da distribuição normal padrão acumulada. 
APÊNDICE A 


\section{Apêndice B}

\section{B.1 Estimação por máxima verossimilhança aproximada}

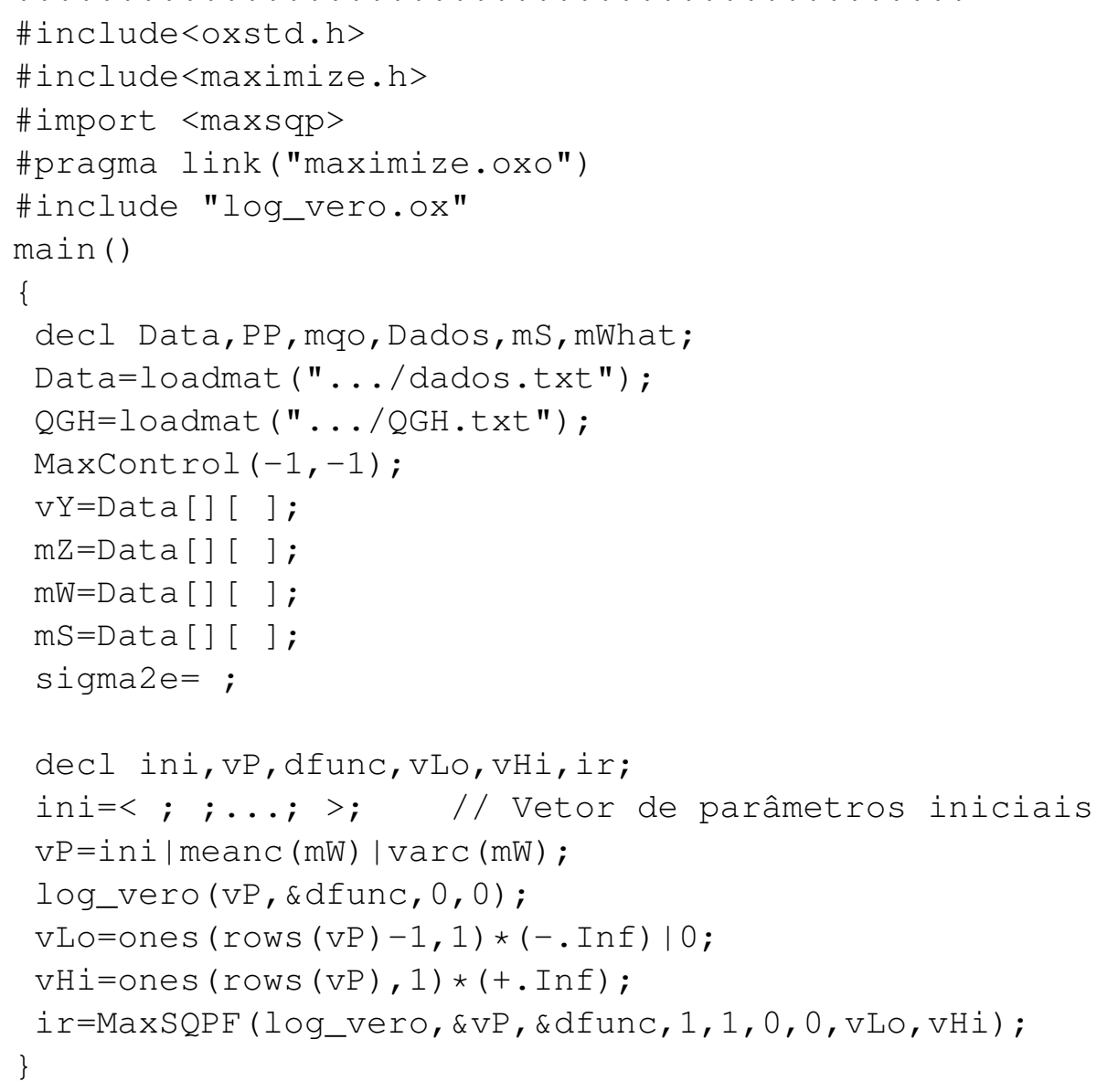

A função log_vero.ox, presente no algoritmo acima, pode ser encontrada no Apêndice B.10.

\section{B.2 Estimação por máxima pseudo-verossimilhança aproximada}

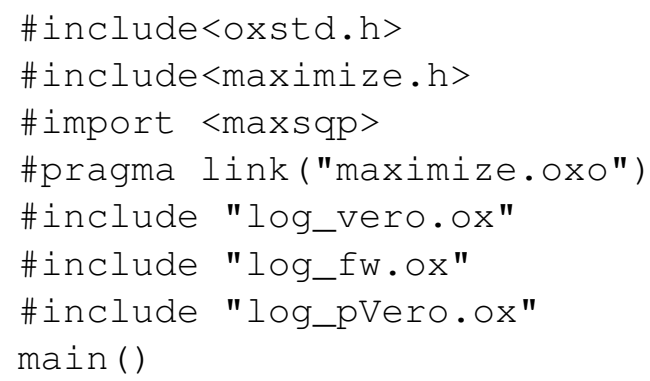




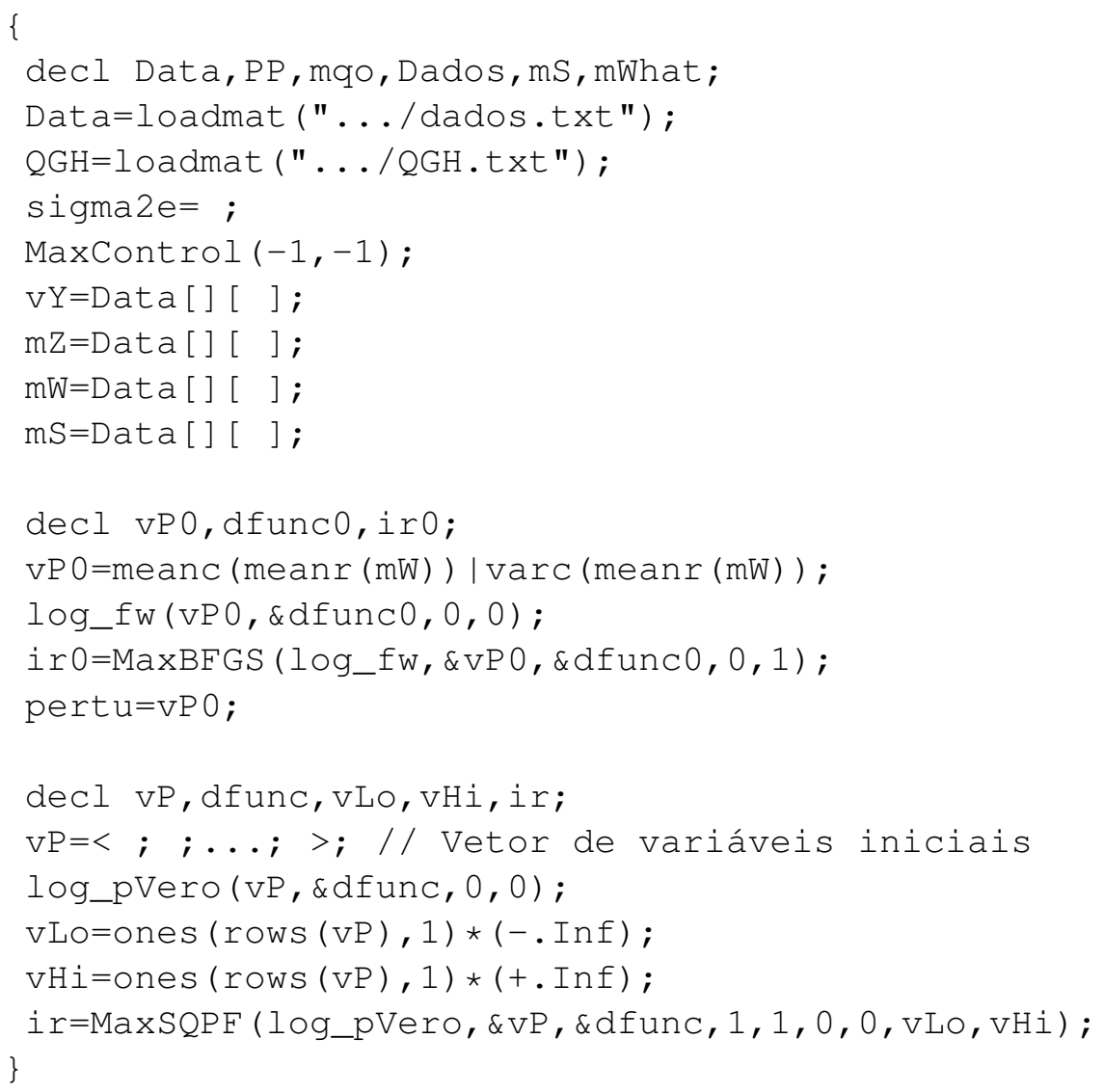

Neste programa são utilizadas as funções log_fw.ox e log_pVero.ox dadas nos Apêndice B.11 e B.12, respectivamente.

\section{B.3 Estimação por calibração da regressão}

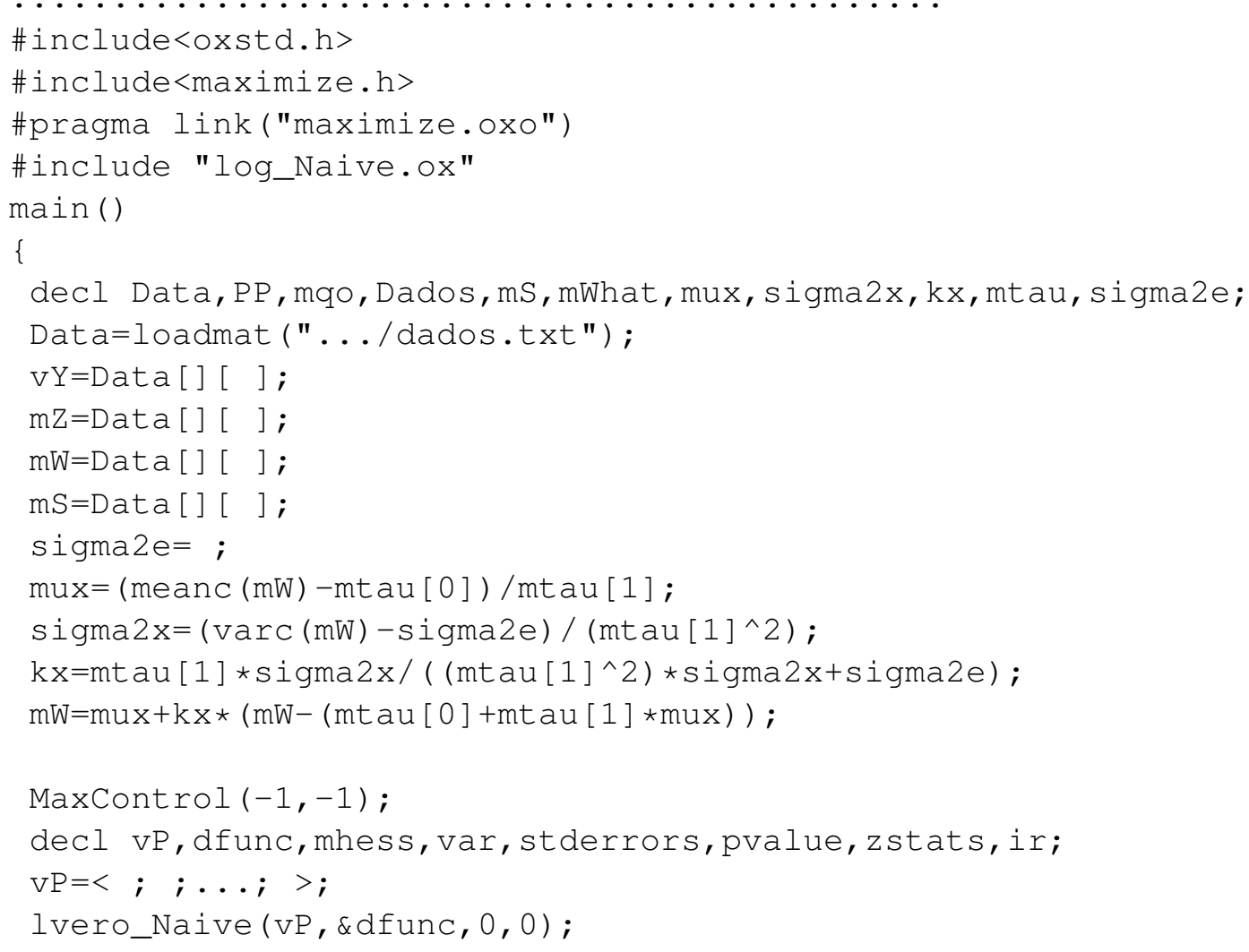


ir=MaxBFGS ( Ivero_Naive, \&vP, \&dfunc, 0, 1);

\}

B.4 Matriz de variâncias e covariâncias dos estimadores de máxima verossimilhança aproximada

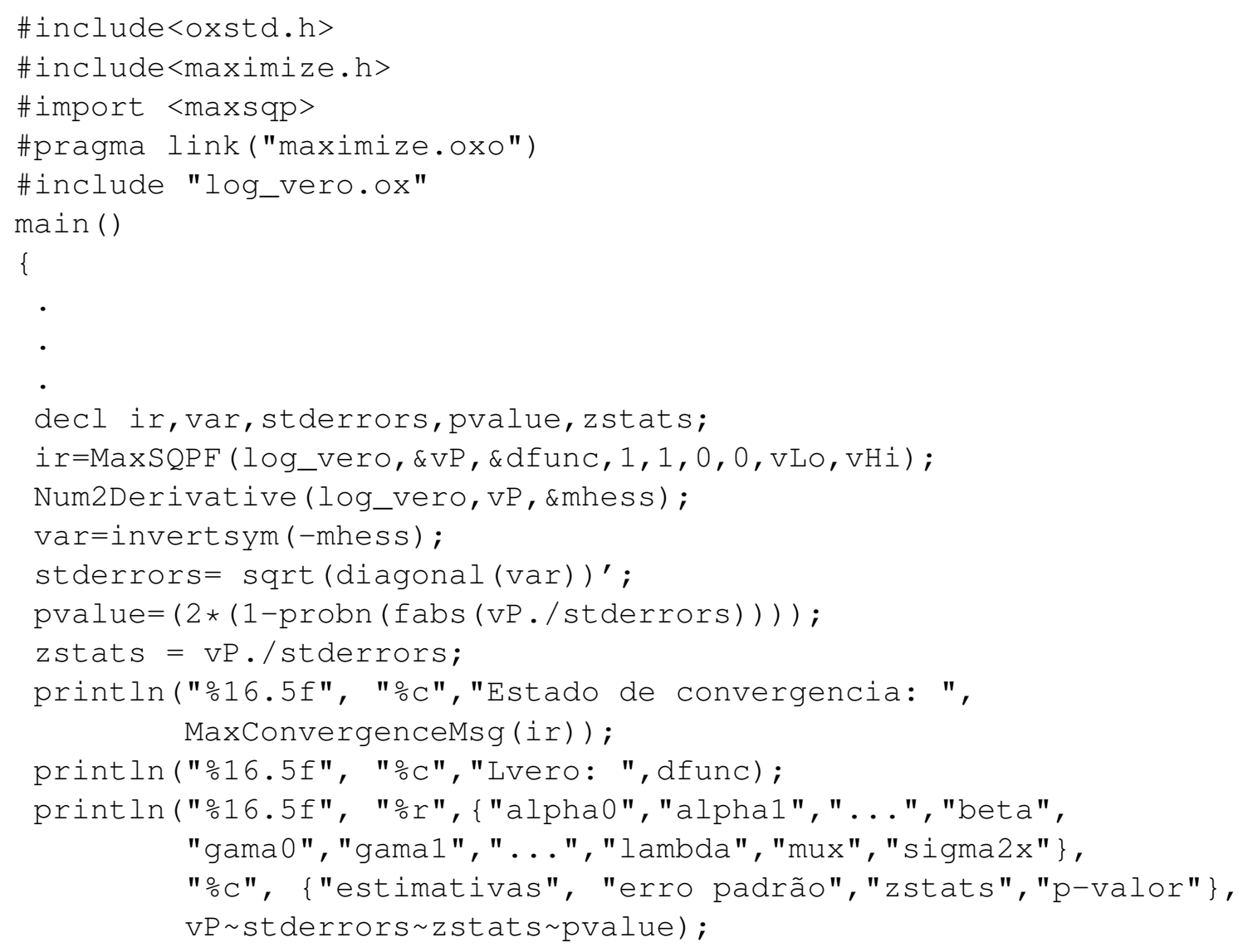

Utilizamos a função Num2Derivative(·) no trecho do código para calcular numericamente a matriz das derivadas segundas da função log_vero.ox com respeito aos parâmetros.

\section{B.5 Matriz de variâncias e covariâncias dos estimadores de máxima pseudo- verossimilhança aproximada}

\#include<oxstd.h>

\#include<maximize.h>

\#import <maxsqp>

\#pragma link ("maximize.oxo")

\#include "log_vero.ox"

\#include "log_fw.ox"

\#include "log_pVero.ox"

main ()

\{

$\cdot$

-

decl ir, mA, vSb, mQ, w, y, q, cont, yest, muest, G, vP00,b;

ir=MaxSQPF ( log_pVero, \&vP, \&dfunc, 1, 1, 0, 0,vLo, vHi ) ; 


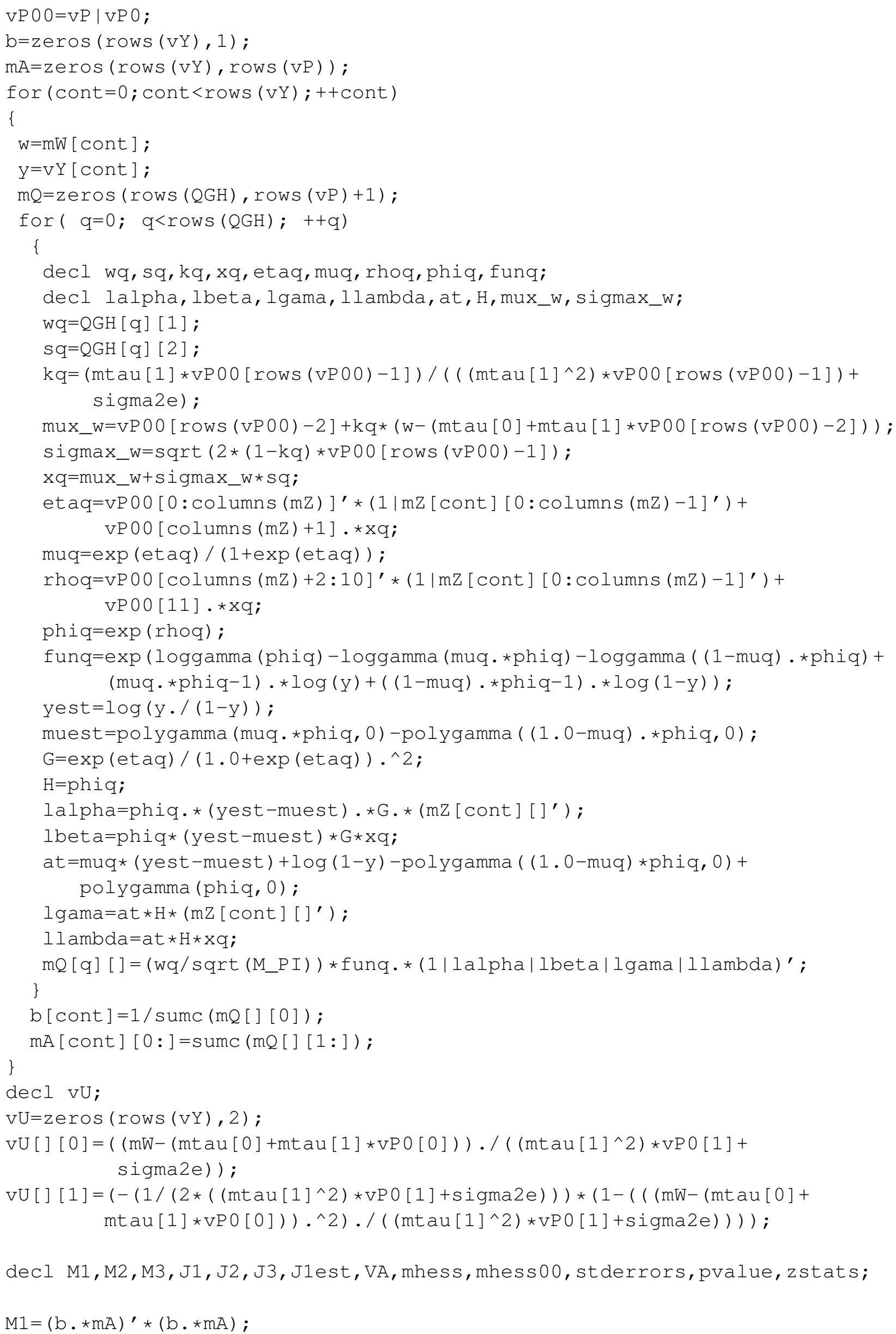




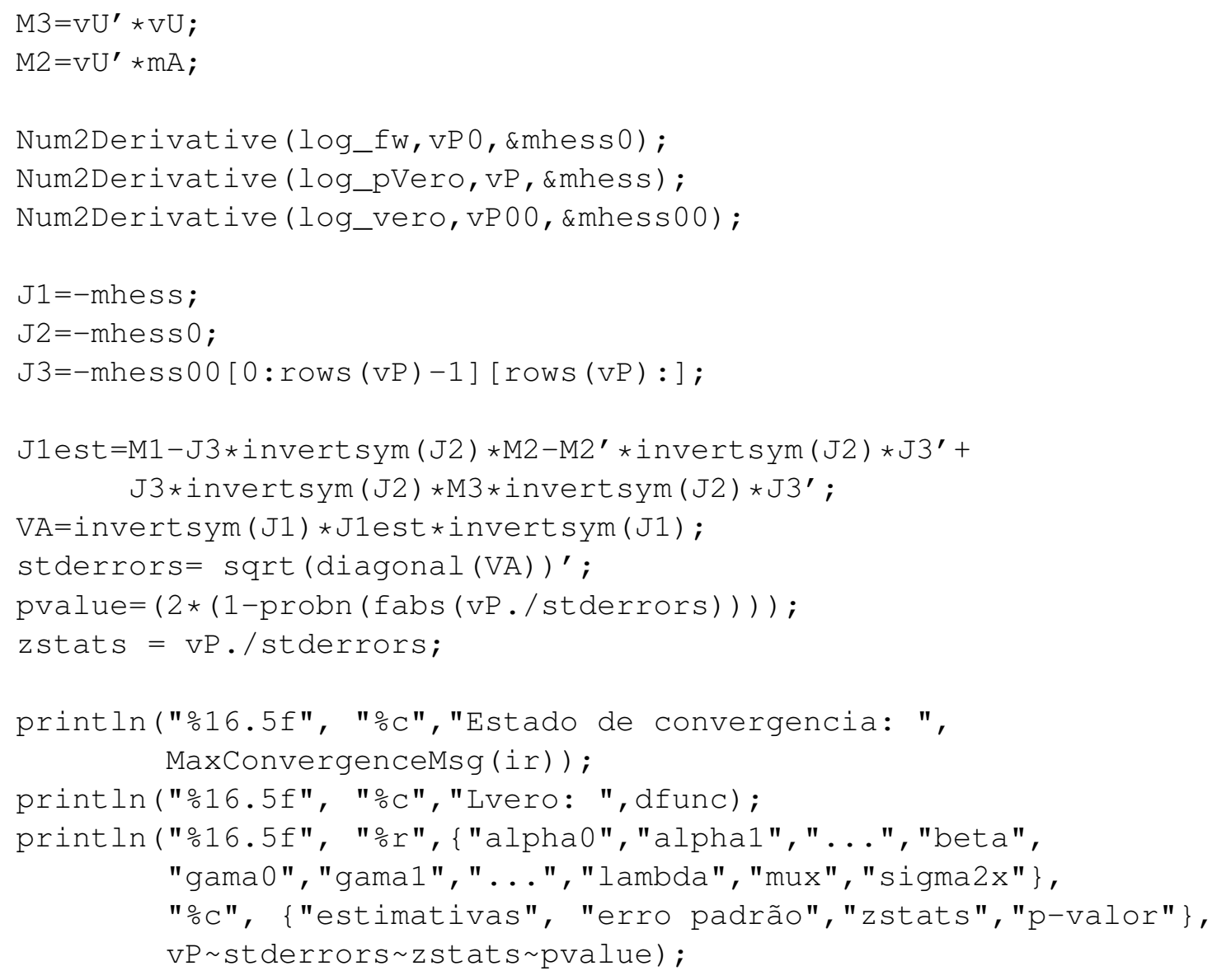

\section{B.6 Matriz de variâncias e covariâncias dos estimadores de calibração da re- gressão}

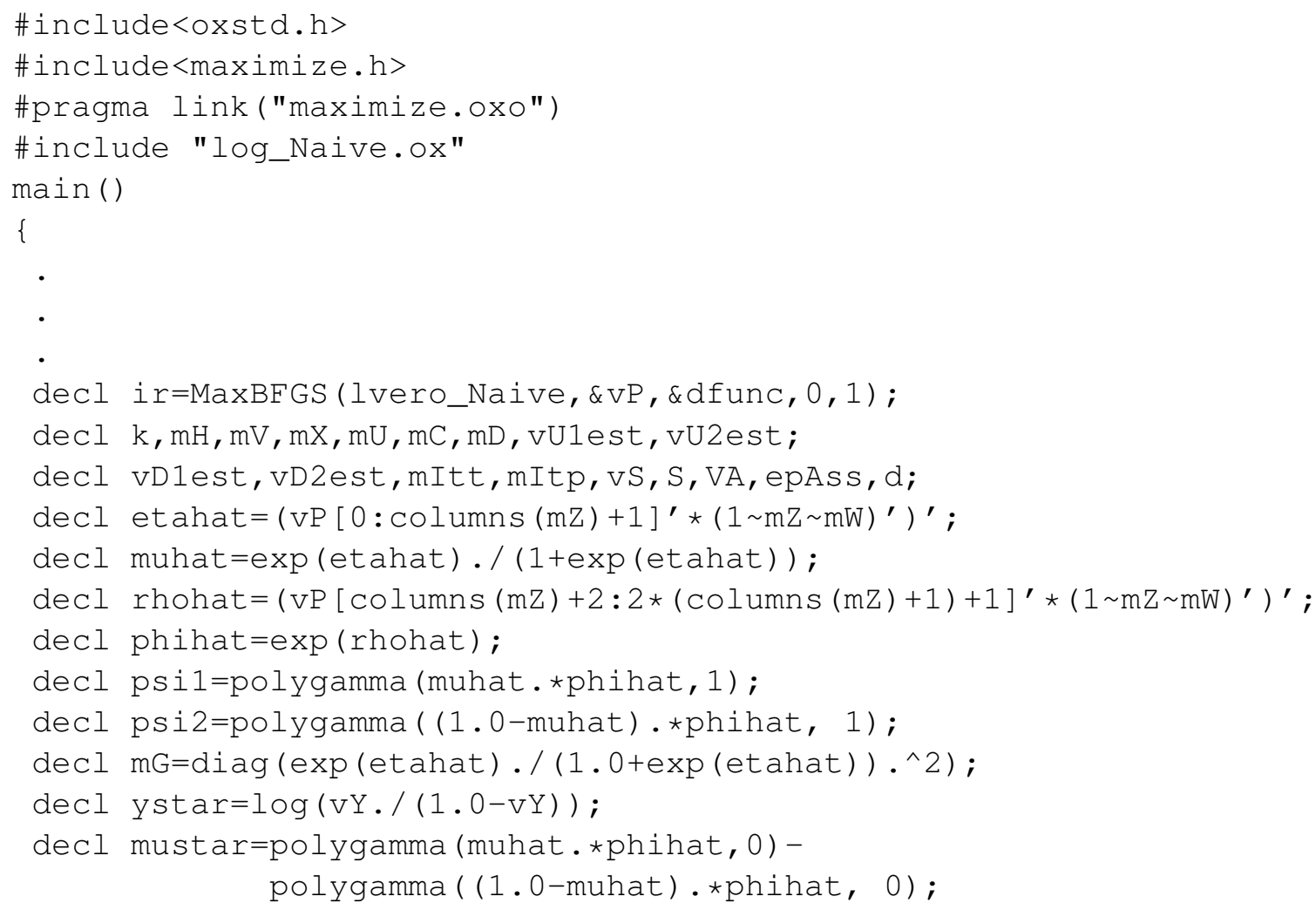




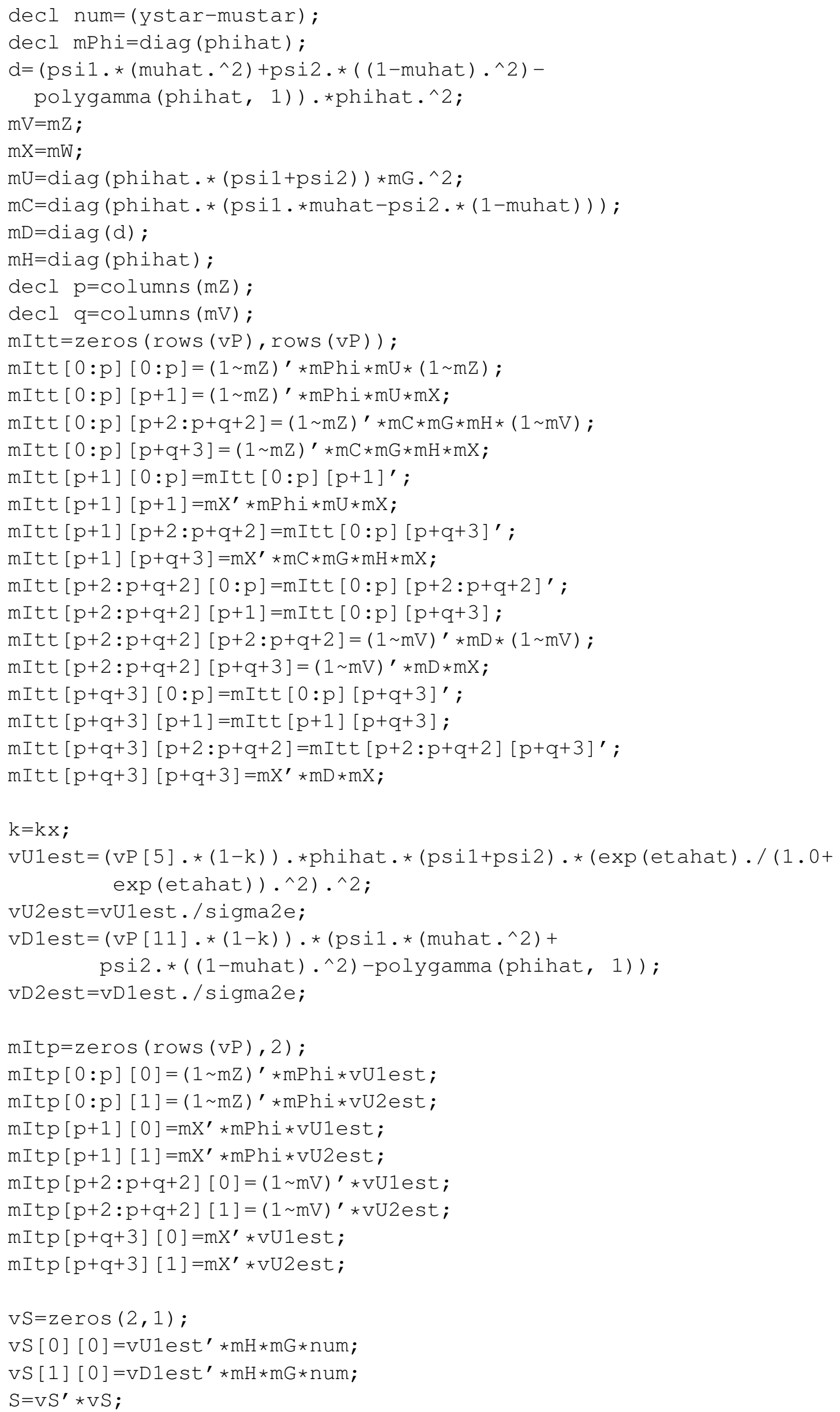




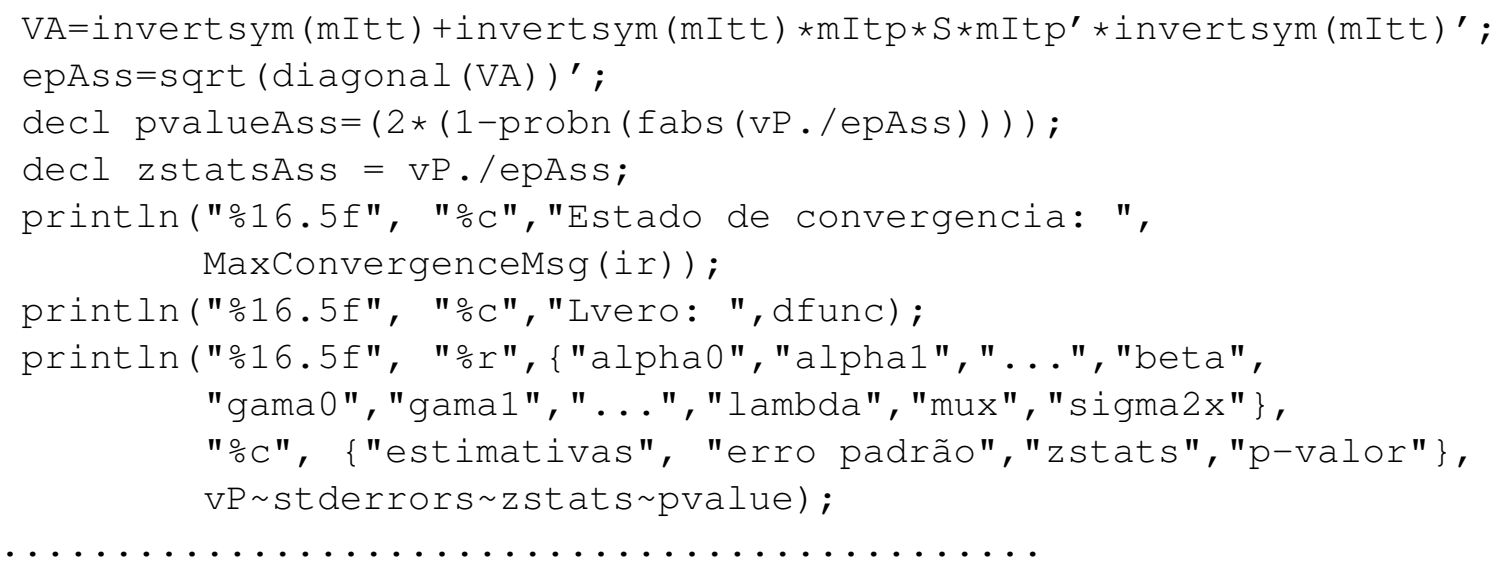

\section{B.7 Resíduos ponderados padronizados}

decl etahat, muhat, phihat, rhohat,psi1,psi2, T, M;

decl V, Gr,WW, tempinv, H, ystar, mustar, h, num, den, rp2;

etahat $=\left(\mathrm{vP}[0: \operatorname{columns}(\mathrm{mZ})+1]^{\prime} *(1 \sim \mathrm{mZ} \sim \mathrm{mW})^{\prime}\right)^{\prime}$;

muhat $=\exp ($ etahat $) . /(1+\exp ($ etahat $))$;

rhohat $\left.=\left(\mathrm{vP}[\mathrm{columns}(\mathrm{mZ})+2: 2 *(\operatorname{columns}(\mathrm{mZ})+1)+1]^{\prime} \star(1 \sim \mathrm{mZ} \sim \mathrm{mW})\right)^{\prime}\right)^{\prime}$;

phihat $=\exp$ (rhohat) ;

psil=polygamma (muhat . *phihat, 1$)$;

psi2=polygamma $((1.0-$ muhat $) . *$ phihat, 1$)$;

$\mathrm{T}=\operatorname{diag}\left(\exp (\right.$ etahat $) . /(1.0+\exp ($ etahat $\left.)) \cdot{ }^{\wedge} 2\right)$;

$\mathrm{M}=\operatorname{diag}($ phihat .* $(\mathrm{psi} 1+\mathrm{psi} 2)) * \mathrm{~T} \cdot{ }^{\wedge} 2$;

$\mathrm{v}=$ psi1+psi2;

Gr=diag (phihat);

$\mathrm{WW}=1 \sim \mathrm{mZ} \sim \mathrm{mW}$;

tempinv=invertsym $\left(\mathrm{WW}^{\prime} \star \mathrm{Gr} * \mathrm{M} * \mathrm{WW}\right)$;

$\mathrm{H}=\operatorname{sqrt}(\mathrm{M} * \mathrm{Gr}) * \mathrm{WW} *$ tempinv $* \mathrm{WW}^{\prime} * \operatorname{sqrt}(\mathrm{M} * \mathrm{Gr})$;

ystar $=\log (\mathrm{vY} . /(1.0-\mathrm{vY}))$;

mustar=polygamma (muhat. *phihat, 0)-polygamma ( (1.0-muhat) $*$ phihat, 0$)$;

h=diagonal $(\mathrm{H})^{\prime}$;

num $=($ ystar-mustar $)$;

den $=(v \cdot *($ ones $(\operatorname{rows}(v \mathrm{~V}), 1)-\mathrm{h})) \cdot{ }^{\wedge} 0.5$;

rp2 $=$ num.$/$ den;

\section{B.8 Análise de influência global}

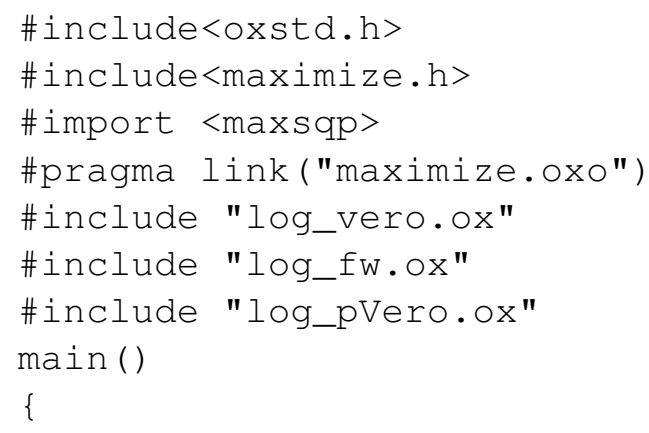




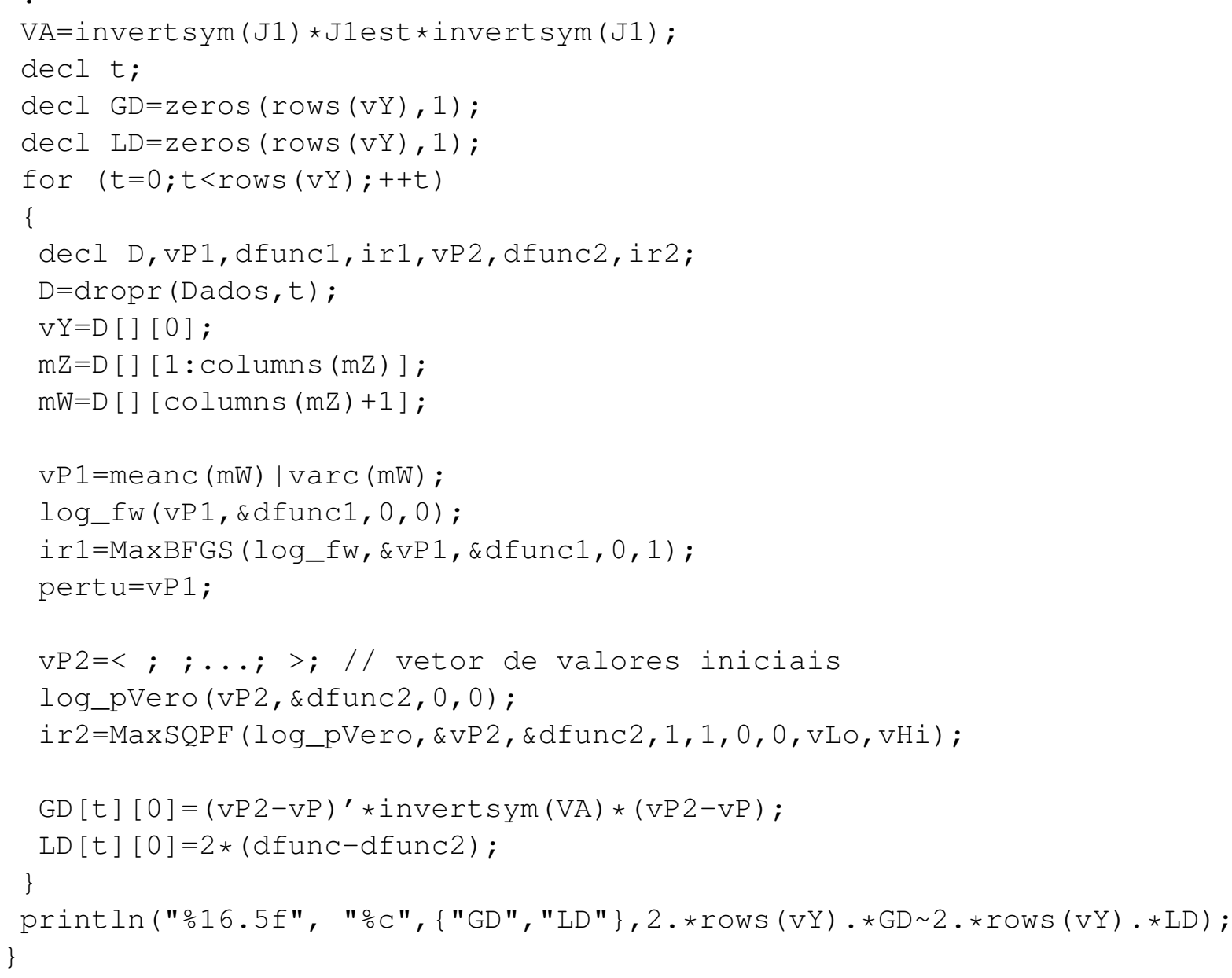

\section{B.9 Análise de influência local}

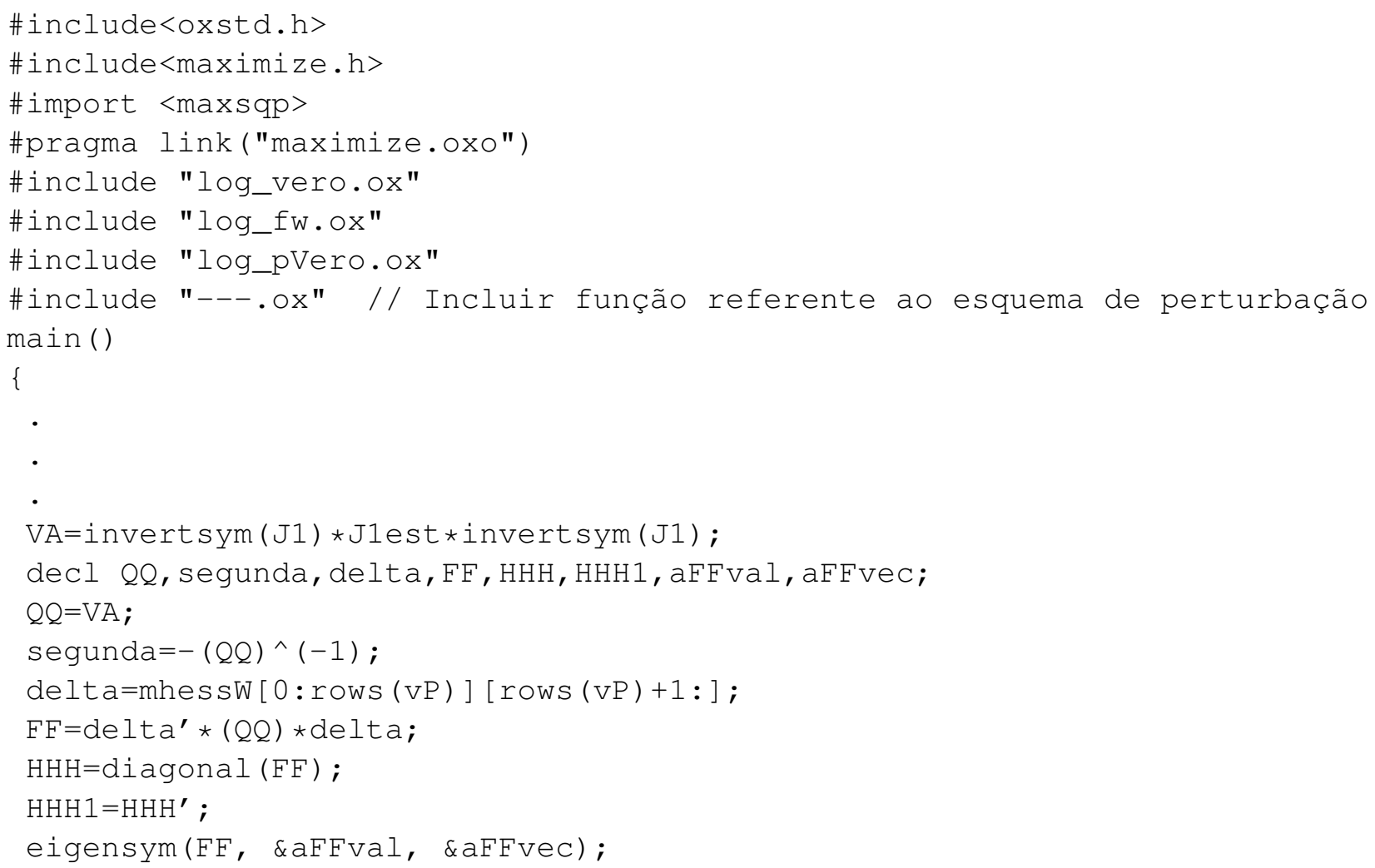




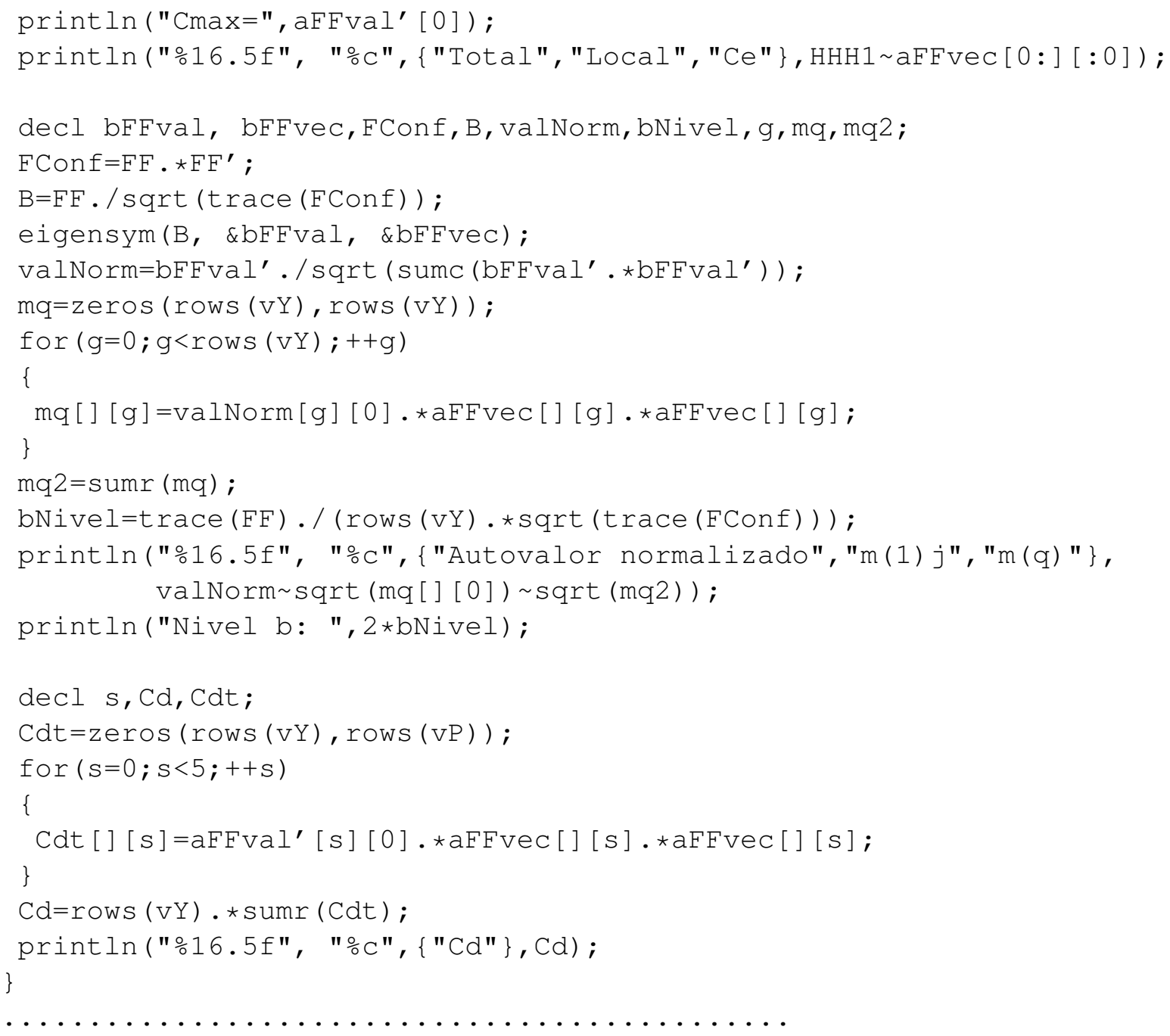

No programa acima, apresentamos os passos para encontrar as medidas de influência local. Dependendo do esquema de perturbação substitua-se no programa a a função --- . ox pelas funções dadas no Apêndice B.14, B.15 ou B.16.

\section{B.10 Logaritmo da função de verossimilhança aproximada}

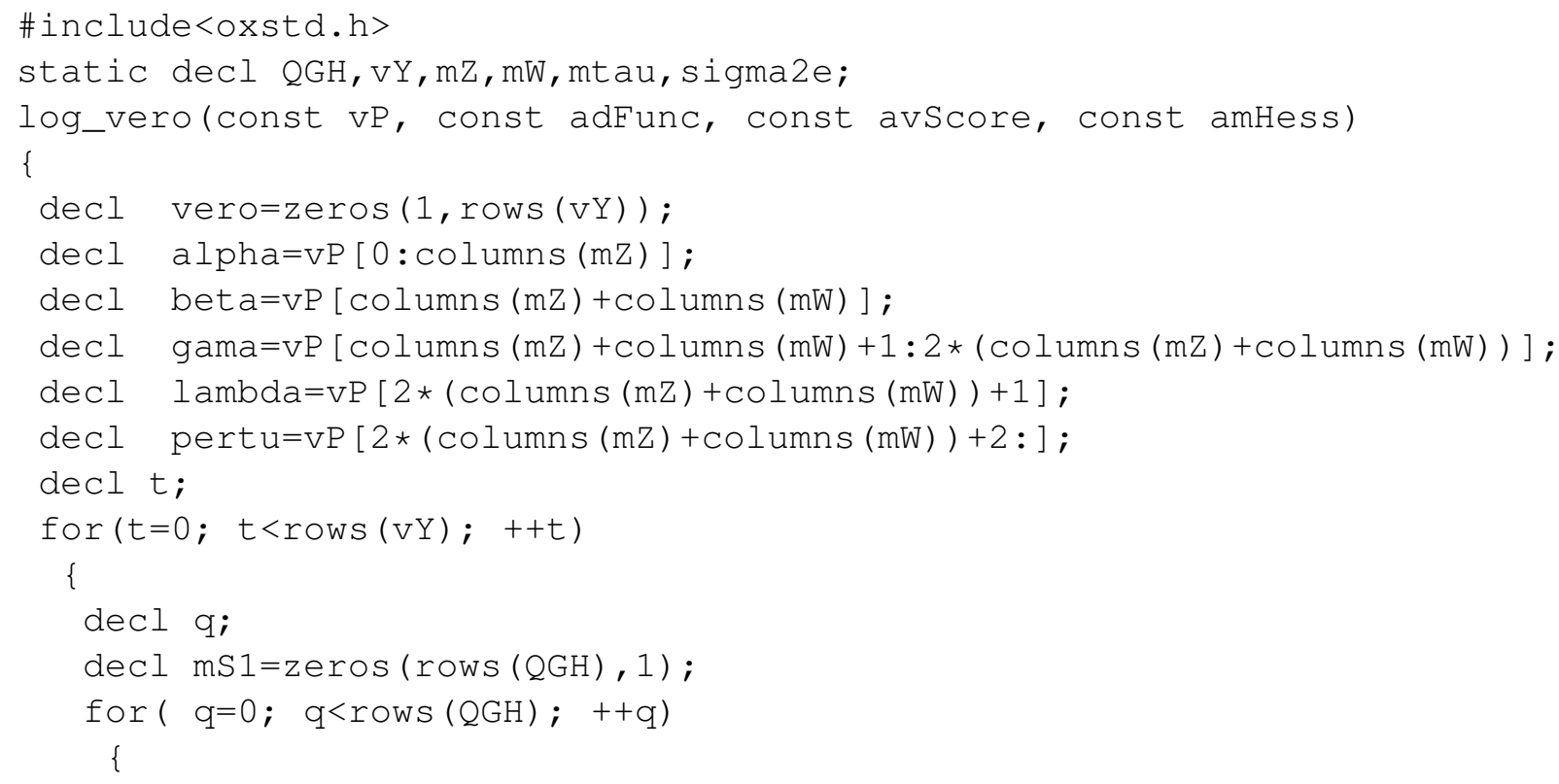




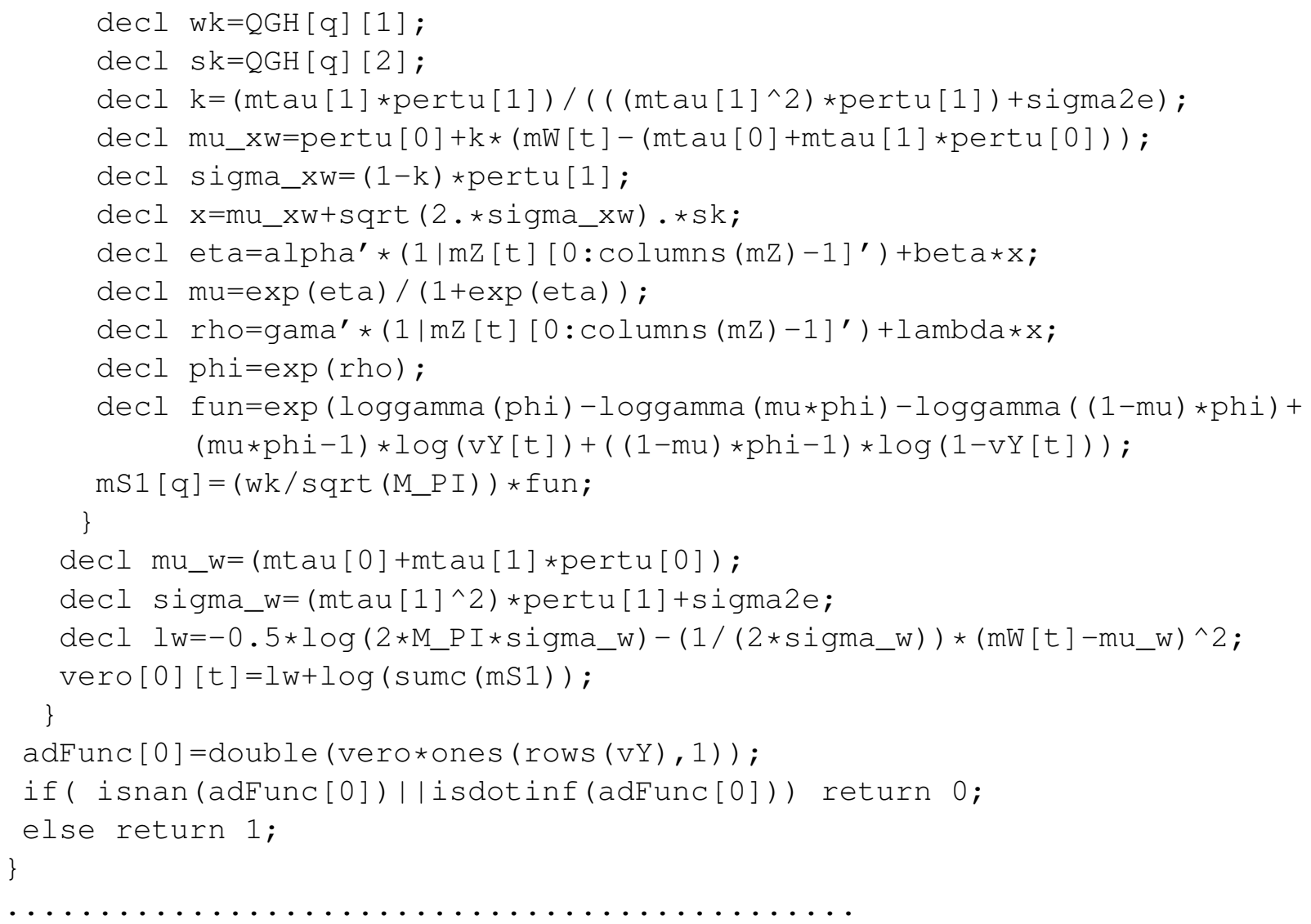

\section{B.11 Logaritmo da função de verossimilhança restrita}

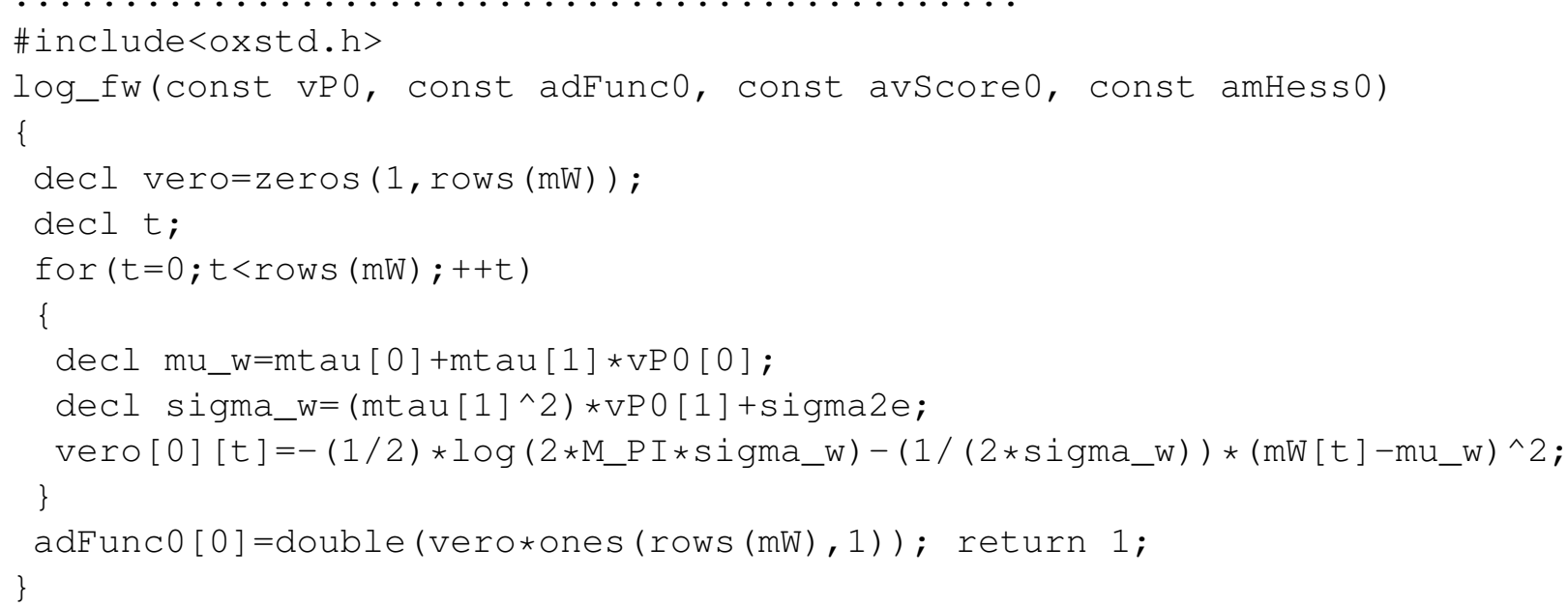

\section{B.12 Logaritmo da função de pseudo-verossimilhança aproximada}

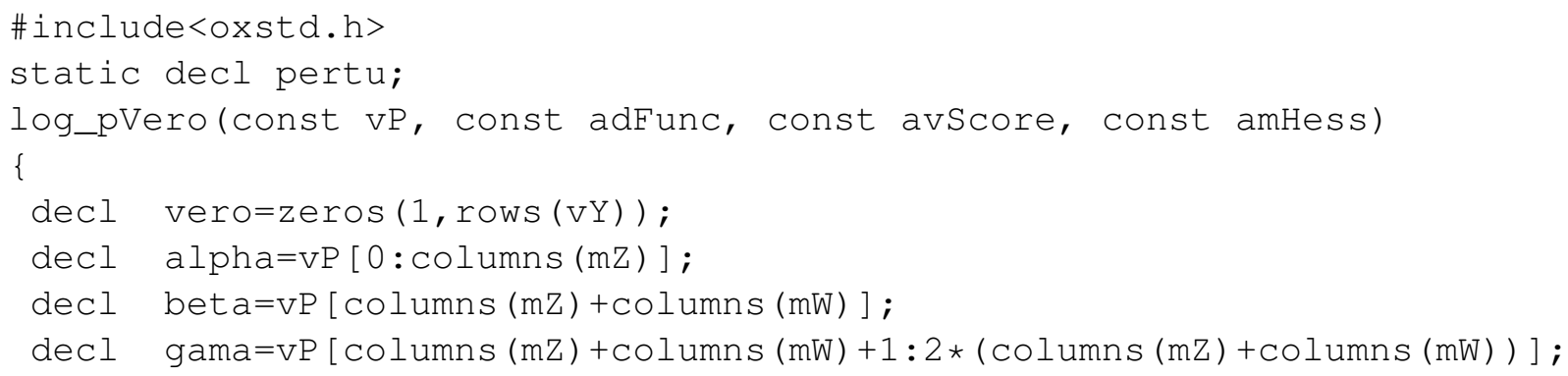




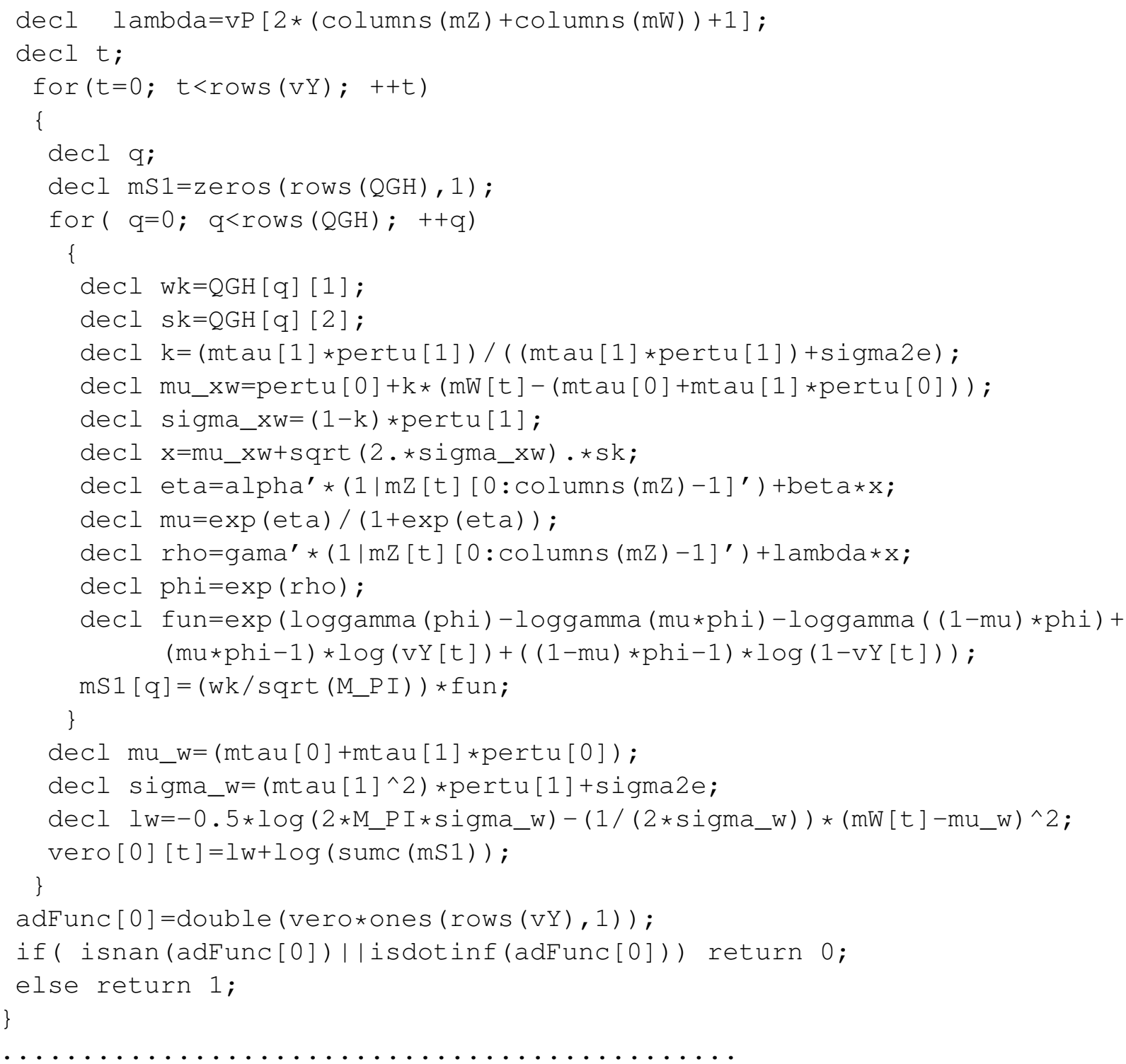

\section{B.13 Logaritmo da função de verossimilhança Naïve ou calibração da regressão}

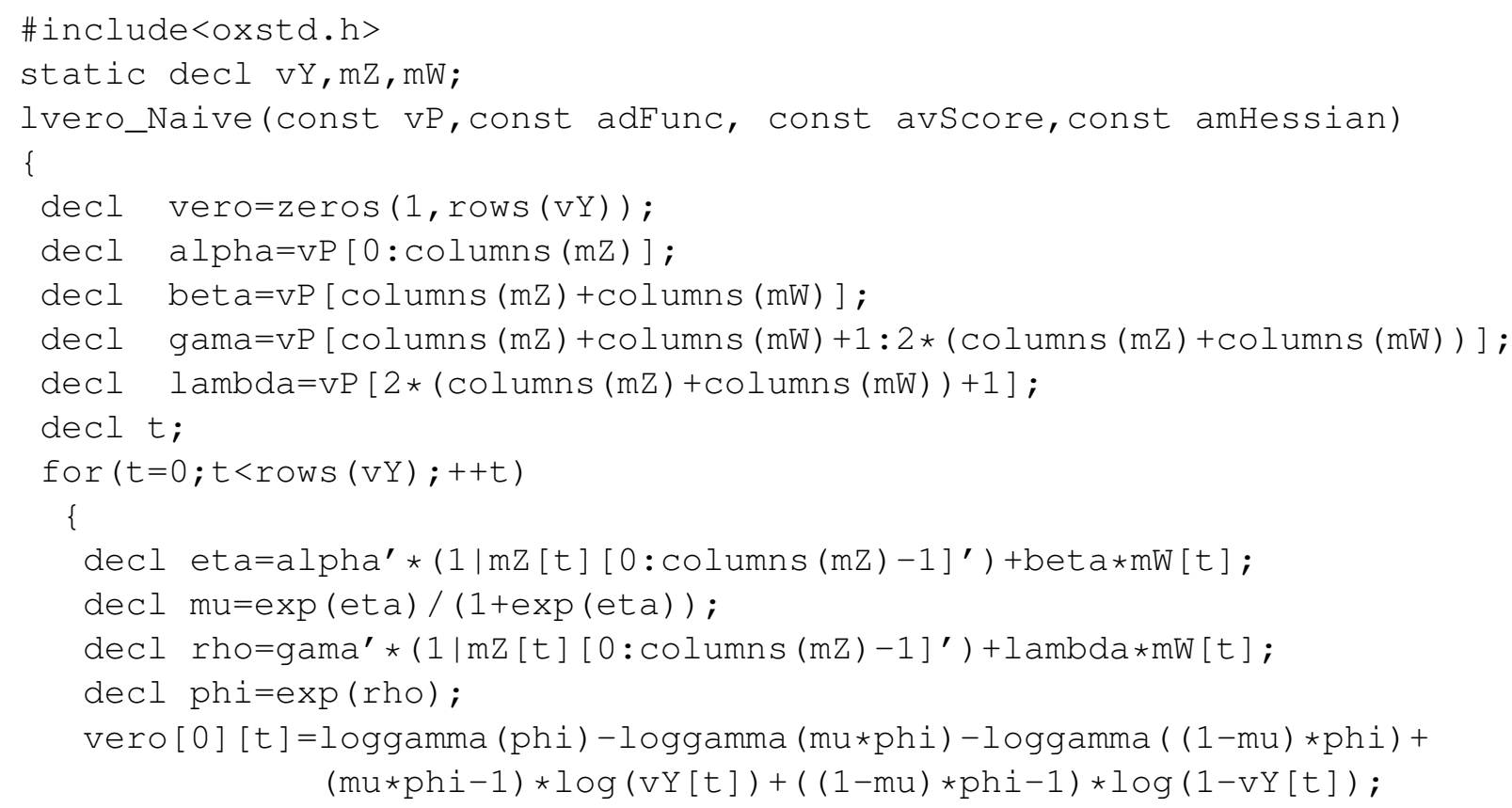




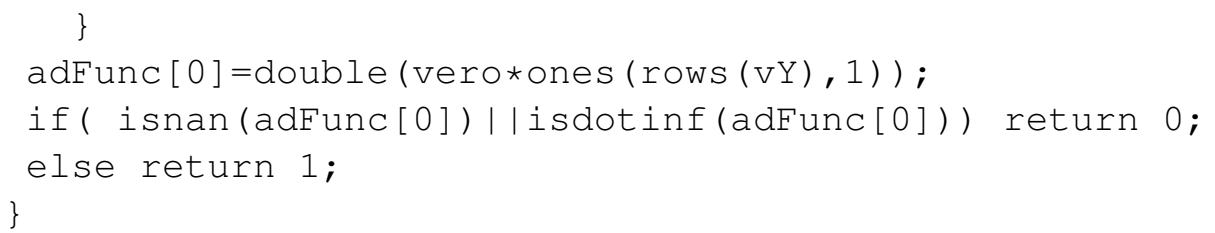

\section{B.14 Logaritmo da função de pseudo-verossimilhança aproximada: Ponderação} de casos

\#include<oxstd.h>

log_pVeroW(const vP, const adFunc, const avScore, const amHess) 


\section{B.15 Logaritmo da função de pseudo-verossimilhança aproximada: Perturbação da variável resposta}

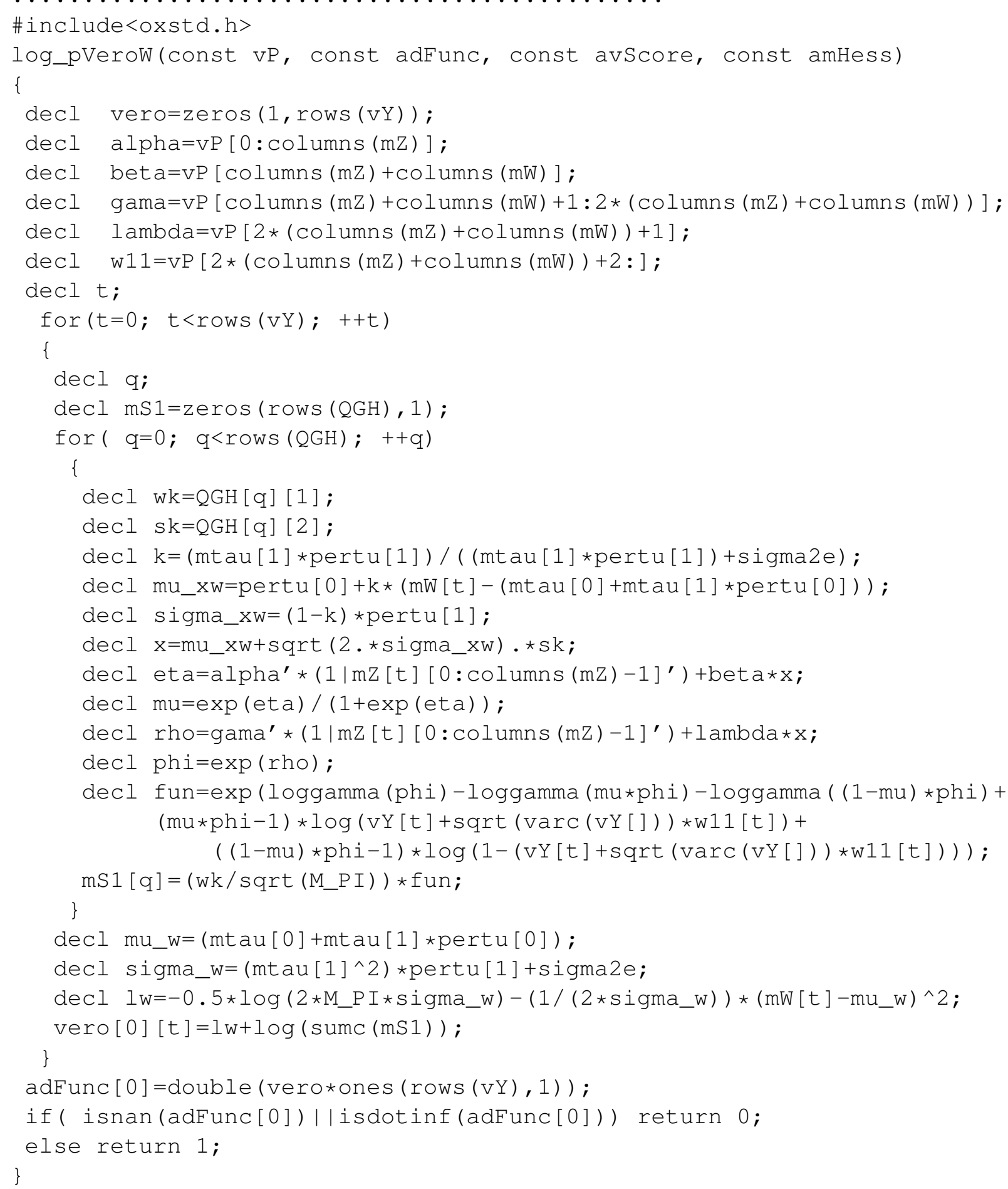

B.16 Logaritmo da função de pseudo-verossimilhança aproximada: Perturbada da variável preditora com erro

\#include<oxstd. h>

log_pVerow (const vP, const adFunc, const avScore, const amHess) 


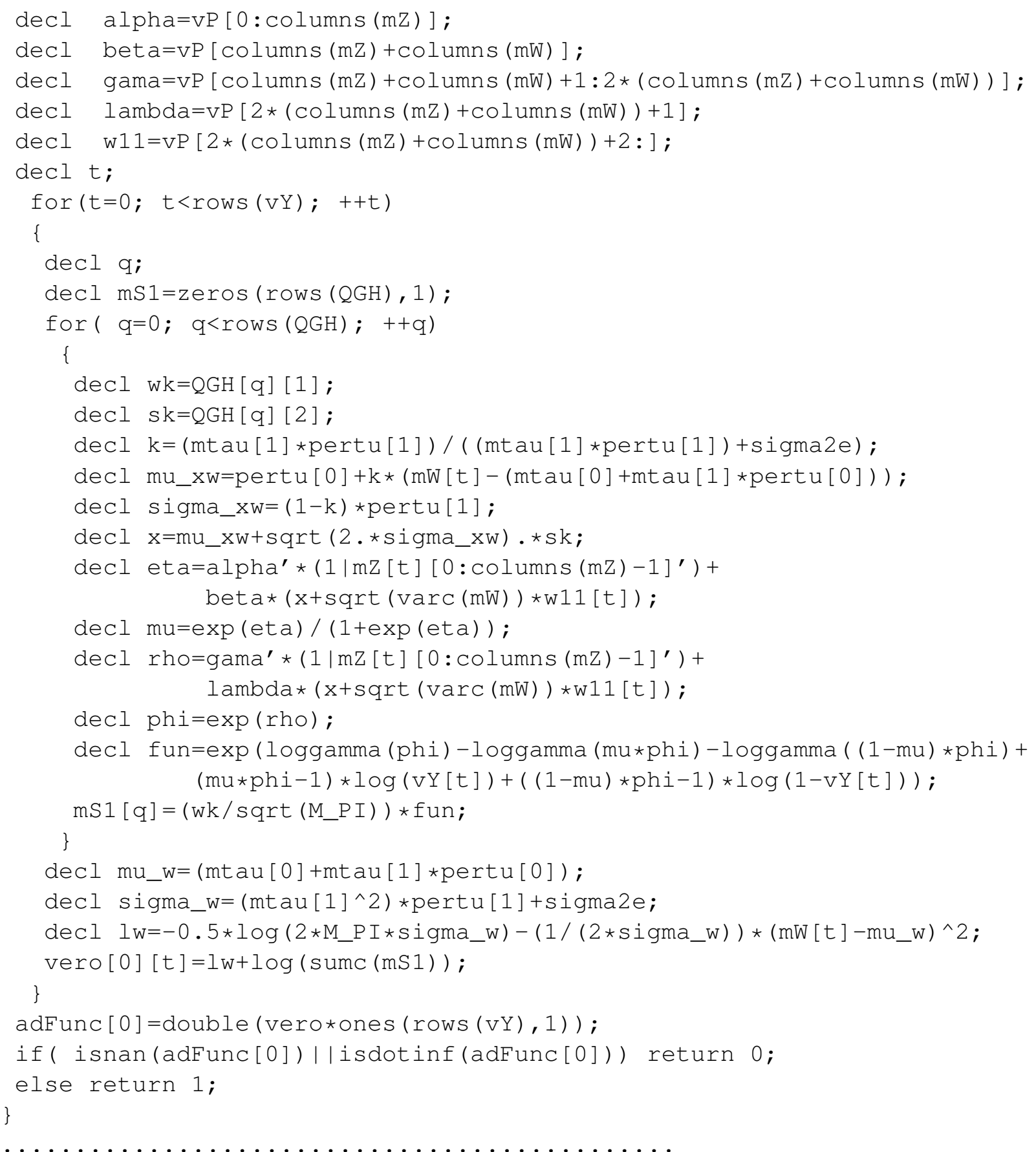




\section{Referências Bibliográficas}

Abramowitz, M. e Stegun, I. (1972). Handbook of Mathematical Functions. New York:Dover.

American College of Surgeons (2008). ATLS Advanced Trauma Life Support Program for Doctors. Chicago: American College of Surgeons.

Armstrong, B. (1985). Measurement error in the generalized linear models. Communications in Statistics B - Simulation and Computation, 14, 529-544.

Atkinson, A. C. (1985). Plots, Transformations and Regression: An Introduction to Graphical Methods of Diagnostic Regression Analysis. New York: Oxford University Press.

Barndorff-Nielsen, O. e Jørgensen, B. (1991). Some parametric models on the simplex. Journal of Multivariate Analisys, 39, 106-116.

Benetos, A., Thomas, F., Joly, L., Blacher, J., Pannier, B., Labat, C., Salvi, P., Smulyan, H., e Safar, M. (2010). Pulse pressure amplification a mechanical biomarker of cardiovascular risk. Journal of American College of Cardiology, 55, 1032-1037.

Buonaccorsi, J. P. (2010). Measuremenet Error: Models, Methods and Applications. London: Chapman and Hall.

Buonaccorsi, J. P. e Tosteson, T. D. (1993). Correcting for nonlinear measurement errors in the dependent variable in the general linear model. Communications in Statistics - Theory and Methods, 22, 2687-2702.

Carroll, R., Gail, M., e Lubin, J. (1993). Case-control studies with errors in covariates. Journal of the American Statistical Association, 88, 185-199.

Carroll, R., Ruppert, D., Stefanski, L., e Crainiceanu, C. M. (2006). Measurement Error in Nonlinear Models: A Modern Perspective. New York: Chapman and Hall.

Carroll, R. J. e Ruppert, D. (1988). Transformation and Weighting in Regression. London: Chapman and Hall.

Carroll, R. J. e Spiegelman, C. H. (1992). Diagnostics for nonlinearity and heteroscedasticity in errors-in-variables regression. Technometrics, 34, 186-196.

Cheng, C. e Van Ness, J. (1999). Statistical Regression with Measurement Error. London: Oxford University Press.

Cook, R. D. (1977). Detection of influential observations in linear regression. Technometrics, 19, $15-18$. 
Cook, R. D. (1986). Assessment of local influence. Journal of the Royal Statistical Society B, 48, 133-169.

Cox, C. (1996). Nonlinear quasi-likelihood models: applications to continuous proportions. Computational Statistics and Data Analysis, 21, 449-461.

Cox, D. e Hinkley, D. (1974). Theoretical Statistics. London: Chapman \& Hall.

Cox, D. e Snell, E. (1968). A general definition of residuals. Journal of the Royal Statistical Society $B, \mathbf{3 0}, 248-275$.

Cox, D. R. (1975). Partial likelihood. Biometrika, 62, 269-276.

Cramér, H. (1999). Mathematical Methods of Statistics. New York:Princeton University Press.

Cribari-Neto, F. e Zeiles, A. (2010). Beta regression in R. Journal of Statistical Software, 34, 1-24.

Delgado, J. J. F. (1995). Estimação por Pseudo Máxima Verossimilhanca. Dissertação, Universidade de São Paulo.

Dolby, G. R. (1976). The ultrastrutural relation: a synthesis of the functional and strutural relation. Biometrika, 63, 39-50.

Doornik, J. (2011). Ox 6.2: Object-Oriented Matrix Programming Language. London: Timberlake Consultants and Oxford: http://www.doornik.com.

Efron, B. (1979). Bootstrap methods: Another look at the jackknife. The Annals of Statistics, 7, $1-26$.

Efron, B. e Tibshirani, R. J. (1994). An Introduction to the Bootstrap. New York: Chapman and Hall.

Espinheira, P., Ferrari, S., e Cribari-Neto, F. (2008a). Influence diagnostics in beta regression. Computational Statistics and Data Analysis, 52, 4417-4431.

Espinheira, P., Ferrari, S., e Cribari-Neto, F. (2008b). On beta regression residuals. Journal of Applied Statistics, 35, 407-419.

Ferrari, S. e Cribari-Neto, F. (2004). Beta regression for modelling rates and proportion. Journal of Applied Statistics, 31, 799-815.

Freedman, L., Midthune, D., Carroll, R., e Kipnis, V. (2008). A comparison of regression calibration, moment reconstruction and imputation for adjusting for covariate measurement error in regression. Statistics in Medicine, 27, 5195-5216.

Fuller, W. A. (1987). Measurement Error Models. New York: John Wiley.

Geys, H., Molenberghs, G., e Ryan, L. M. (1999). Pseudolikelihood modeling of multivariate outcomes in developmental toxicology. Journal of the American Statistical Association, 94, 734-745.

Godambe, V. P. (1976). Conditional likelihood and unconditional optimum estimating equations. Biometrika, 63, 277-284. 
Godambe, V. P. (1991). Estimating Functions. New York: Clarendon Press.

Golub, G. H. e Welsch, J. (1967). Calculation of Gauss quadrature rules. Stanford University, Technical Report 81.

Gong, G. e Samaniego, F. (1981). Pseudo maximum likelihood estimation: theory and applications. The Annals of Statistics, $\mathbf{9}, 861-869$.

Guolo, A. (2011). Pseudo-likelihood inference for regression models with misclassified and mismeasured variables. Statistica Sinica, 21, 1639-1663.

Gupta, A. K. e Nadarajah, S. (2004). Handbook of Beta Distribution and Its Applications. New York: Marcel Dekker.

Hayakawa, T. e Puri, M. (1985). Asymptotic expansions of the distributions of some test statistics. Annals of the Institute of Statistical Mathematics A, 62, 451-460.

Kendall, M. G. e Stuart, A. (2009). The Advanced Theory of Statistics. London: Griffin.

Kieschnick, R. e McCullough, B. (2003). Regression analysis of variates observed on (0,1): percentages, proportions and fractions. Statistical Modelling, 3, 193-213.

Lemonte, A. J. e Ferrari, S. (2011a). Size and power properties of some tests in the BirnbaumSaunders regression model. Computational Statistics and Data Analysis, 55, 1109-1117.

Lemonte, A. J. e Ferrari, S. (2011b). Testing hypotheses in the Birnbaum-Saunders distribution under type-II censored samples. Computational Statistics and Data Analysis, 55, 2388-2399.

Lemonte, A. J. e Ferrari, S. (2012). The local power of the gradient test. Annals of the Institute of Statistical Mathematics, 64, 373-381.

Lesaffre, E. e Verbeke, G. (1998). Local influence in linear mixed models. Biometrics, 54, 570-582.

Liang, K.-Y. e Self, S. G. (1996). On the asymptotic behaviour of the pseudolikelihood ratio test statistic. Journal of the Royal Statistical Society, B, 58, 785-796.

Liu, Q. e Pierce, D. (1994). A note on Gauss-Hermite quadrature. Biometrika, 81, 624-629.

Louis, T. (1982). Finding the observed information matrix when using the EM algorithm. Journal of the Royal Statistical Society B, 44, 226-133.

McCullagh, P. (1987). Tensor Methods in Statistics. London: Chapman and Hall.

McCullagh, P. e Nelder, J. (1999). Generalized Linear Models. London: Chapman and Hall.

Moran, P. (1971). Estimating strutural and functional relationships. Jounal of Multivariate Analysis, 1, 232-255.

Murrell, P. (2006). R Graphics. London: Chapman and Hall.

Ospina, R. M. (2008). Modelo de Regressão Beta Inflacionados. São Paulo: Tese do Programa de Pós-Graduação em Estatística, Instituto de Matemática e Estatística da Universidade de São Paulo. 
Pace, L. e Salvan, A. (1997). Principles of Statistical Inference from a Neo-Fisherian Perspective. London: World Scientifie Publishing.

Papke, L. e Wooldridge, J. (1996). Econometric methods for fractional response variables with an application to 401(k) plan participation rates. Journal of Applied Econometrics, 11, 619-632.

Parke, W. R. (1986). Pseudo maximum likelihood estimation: the asymptotic distribution. Annals of Statistics, 14, 335-357.

Peixoto, L. (2008). Quadratura de Gauss Iterativa com Base nos Polinômios Ortogonais Clásicos. Belo Horizonte: Dissertação do Programa de Pós-Graduação em Modelagem Matemática e Computacional, Centro Federal de Educação Tecnológica de Minas Gerais.

Poon, W. Y. e Poon, Y. S. (1999). Conformal normal curvature and assessment of local influence. Journal of the Royal Statistical Society B, 61, 51-61.

Pregibon, D. (1981). Logistic regression diagnostics. The Annals of Statistics, 9, 705-724.

Rabe-Hesketh, S., Skrondal, A., e Pickles, A. (2005). Maximum likelihood estimation of limited and discrete dependent variable models with nested random effects. Journal of Econometrics, 128, 301-323.

Rao, C. (1948). Large sample tests of statistical hypotheses corcerning several parameters with applications to problens of estimation. Proceedings of the Cambridge Philosophical Society, 40, $50-57$.

Roeder, K., Carroll, R. J., e Lindsay, B. G. (1996). A semiparametric mixture approach to casecontrole studies with errors in covariables. Journal of the American Statistical Association, 91, $722-732$.

Rosner, W. B. e Spiegelman, D. (1989). Correction of logistic regression relative risk estimate and confidence intervals for systematic within-person measurement error. Statistics in Medicine, $\mathbf{8}$, $1075-1093$.

Simas, A., Barreto-Souza, W., e A.V.Rocha (2010). Improved estimators for a general class of beta regression models. Computational Statistics and Data Analysis, 54, 348-366.

Smithson, M. e Verkuilen, J. (2006). A better lemon-squeezer? maximum-likelihood regression with beta-distributed dependent variables. Psychological Methods, 11, 54-71.

Steen, K. V., Molenberghs, G., Verbeke, G., e Thijs, H. (2001). A local influence approach to sensitivity analysis of incomplete longitudinal ordinal data. Statistical Modelling, 1, 125-142.

Stefanski, L. (1985). The effects of measurement error on parameter estimation. Biometrica, 72, $583-592$.

Stefanski, L. e Carroll, R. (1985). Covariate measurement error in logistic regression. The Annals of Statistics, 13, 1335-1351.

Tanner, M. (1996). Tools for Statistical Inference. New York: Springer. 
Terrell, G. (2002). The gradient statistic. Computing Science and Statistics, 34, 206-215.

Thurston, S., Williams, P., Hauser, R., Hu, H., Hernandez-Avila, M., e Spiegelman, D. (2005). A comparison of regression calibration approaches for designs with internal validation data. Journal of Statistical Planning and Inference, 131, 175-190.

Villegas, C., Paula, G. A., e Leiva, V. (2011). Birnbaum-Saunders mixed models for censored reliability data analysis. IEEE Transactions on Reliability, 60, 748-758.

Wald, A. (1943). Tests of statistical hypostesis concerning several parameters when the number of observations is large. Transactions of the American Mathematical Society, 54, 426-482.

Whittemore, A. e Keller, J. (1988). Approximations for regression with covariate measurement error. Journal of the American Statistical Association, 83, 1057-1066.

Wilks, S. (1938). The large-sample distribution of the likelihood ratio for testing composite hypotesis. Annals of Mathematical Statistics, 9, 60-62.

Williams, D. (1987). Generalized linear models diagnostic using the deviance and single case deletion. Applied Statistics, 36, 181-191.

Zhu, H. e Zhang, H. (2004). A diagnostic procedure based on local influence. Biometrika, 91, 579-589. 